\title{
Pyridyl Phosphine Complexes in the Design of Hydration Catalysts
}

\author{
Rosemarie (Rosie) Janet Somerville
}

\section{Victoria \\ UNIVERSITY OF WELLINGTO
Te Whare Wānanga \\ Te Whare Wānanga
te Ūpoko o te Ika a Māu}

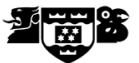

\author{
A thesis \\ submitted to Victoria University of Wellington \\ in fulfilment of the \\ requirements for the degree of \\ Master of Science \\ in Chemistry
}

Victoria University of Wellington 


\section{Abstract}

Recent advances in homogeneous catalysis have identified the importance of ligands able to participate in the catalytic cycle. Particularly relevant to making chemistry "greener" are those ligands that solubilise the catalyst in aqueous solution, and those that are able to activate water molecules towards reaction with the metal complex or substrate. This thesis describes the synthesis and coordination chemistry of a novel ligand bearing 2-pyridylphosphine substituents attached to a 2,6-pyridyl backbone $\left.{ }^{2-p y r} \mathrm{PNP},\left[\left(\mathrm{C}_{5} \mathrm{H}_{4} \mathrm{~N}\right)_{2} \mathrm{PCH}_{2}\right]_{2} \mathrm{C}_{5} \mathrm{H}_{3} \mathrm{~N}\right)$. These components were selected for their abilities to interact with water through dearomatisation processes, hydrogen bonding, and the basic pyridyl nitrogen atoms.

The synthesis of pure ${ }^{2-p y r} \mathrm{PNP}$ described here represents a much improved method for the synthesis of pyridylphosphines compared to those published in the literature. This is demonstrated by comparison with the original synthetic route, which produced many intractable impurities, as well as by the ability of the new method to provide PhPNP from an economical and air-stable starting material.

Reactions of ${ }^{2-p y r}$ PNP with rhodium precursors show complicated reactivity, including the potential formation of paramagnetic species. Investigation into the reactivity of ${ }^{2-p y r} \mathrm{PNP}$ with analogous iridium precursors resulted in the synthesis of $\left[\left({ }^{2-p y r} \mathrm{PNP}\right) \operatorname{Ir}(\operatorname{cod})\right] \mathrm{Cl}$. This is the first crystallographically characterised complex containing a facially coordinated PNP ligand. The cod ligand can be removed with ethene and hydrogen to form bis(ethene) and chloroiridium(III) bis(hydride) complexes [(2-pyr PNP $\left.) \operatorname{Ir}\left(\mathrm{C}_{2} \mathrm{H}_{4}\right)_{2}\right] \mathrm{Cl}$ and $\left[\left({ }^{2-p y r} \mathrm{PNP}\right) \operatorname{Ir}(\mathrm{H})_{2} \mathrm{Cl}\right]$, respectively. Both complexes contain meridionally-coordinated ${ }^{2-p y r} \mathrm{PNP}$.

Preliminary investigations reveal that the iridium complexes are fairly successful nitrile hydration catalysts under aqueous conditions. In addition, the cod and bis(ethene) complexes bearing ${ }^{2-p y r}$ PNP are more active than the cod complex of the pyridyl-free ${ }^{\mathrm{Ph}} \mathrm{PNP}$ ligand. 


\section{Acknowledgements}

To my supervisor Prof. John Spencer, thank you for the fun over the years, from the introductions to organometallic chemistry in CHEM 202 and beyond, to the freedom to explore and find my own way through and around research problems.

Thanks to the members of the research group for your support, particularly Melanie, Teresa, and Kathryn who also worked in LB211. Thanks also to Brad Anderson for the CHEM 306 project on $\mathrm{C}_{6} \mathrm{~F}_{5}$-substituted PCP pincer ligands, the proofreading, and the helpful discussions.

The administrative and technical staff of SCPS have been wonderful. Extra thanks go to Ian Vorster for his patience, kindness, and $600 \mathrm{MHz}$ booking; Jackie King and Teresa Gen for lending me lab equipment and chemicals, and for lending an ear when times were tough; and Maryke Barnard for the chats, encouragement, and banter.

Thanks to my wonderful friends and flatmates for putting up with me spending my time working this last year. Thanks to my SCPS friends for serious and non-serious discussions, broadening my horizons with papers, hugs, cheering me up, ClickHole, cricket conversations, and weekend chemistry company. To those non-chemistry Masters students (Hannah Webster, Shouhei Tanaka, Samantha Morris, and Hannah Hutchinson) who supported me with their kind words of encouragement, I am very grateful.

I would also like to acknowledge support from the Victoria Masters by Thesis Scholarship, Victoria Graduate Award, and Curtis Gordon Research Scholarship.

Finally, the biggest thank you goes to my parents and brother who have supported me for so long. 


\section{Contents}

Abstract i

Acknowledgments

Contents iii

List of Figures vi vi vis

List of Tables vii

List of Schemes viii

Pincer Ligand Terminology and Glossary xi

1 Introduction 1

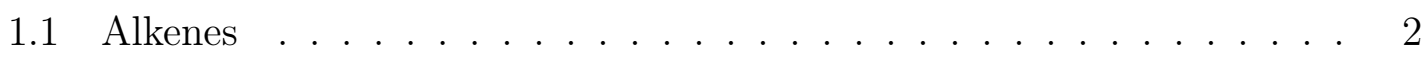

1.2 Alkynes ............................. 4

1.3 Nitriles . . . . . . . . . . . . . . . . . 5

1.4 Elementary Steps . . . . . . . . . . . . . . . . 7

1.4.1 Oxidative Addition . . . . . . . . . . . . . . . 8

1.4 .2 Migration .................... . . . 9

1.4.3 Reductive Elimination . . . . . . . . . . . . . . . . 10

1.5 Catalyst Design . . . . . . . . . . . . . . . . . . . . . 11

1.5.1 Ligands . . . . . . . . . . . . . . . . . . . . 11

1.6 Cooperative Catalysis . . . . . . . . . . . . . . . . . 13

1.6.1 Hydrogen Bonding . . . . . . . . . . . . . . . 14

1.6.2 Pyridylphosphines . . . . . . . . . . . . . 15

1.6.3 Aromatisation-Dearomatisation . . . . . . . . . . 17

1.6.4 Lewis Acidic Sites . . . . . . . . . . . . . . . . . . 17

1.7 Research Objectives . . . . . . . . . . . . . . . . . . . . 19

2 Ligand Synthesis $\quad 20$

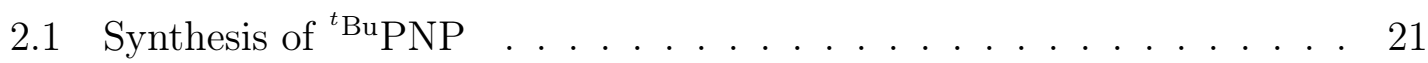

2.2 Synthesis of a Vinyl-Substituted PNP Ligand . . . . . . . . . . . 25

2.2.1 Hydroboration of $\mathrm{Vi}^{\mathrm{PNP}} \ldots \ldots \ldots$. . . . . . . . . . 28

2.3 Synthesis of ${ }^{2-p y r} \mathrm{PNP} \ldots \ldots \ldots$. . . . . . . . . . . . . . . . . . . 29 
2.3.1 Overview . . . . . . . . . . . . . . . . . . . . 29

2.3.2 Step 1: Cleavage of a $\mathrm{P}-(2-$ pyridyl) Bond . . . . . . . . . . . 31

2.3 .3 Step $2 \ldots \ldots \ldots \ldots$

2.3.4 2,2'-Bipyridine . . . . . . . . . . . . . . . 34

2.3.5 Phosphorus $(\mathrm{V}) \ldots \ldots . \ldots . \ldots 36$

2.3.6 Characterisation of 2-pyridylphosphines . . . . . . . . . 37

2.4 Synthesis of ${ }^{\mathrm{Ph}} \mathrm{PNP} \ldots \ldots \ldots \ldots$. . . . . . . . . . . . . . . . 38

2.5 Phosphine Selenides . . . . . . . . . . . . . . . . . . . . . . 39

2.6 Concluding Remarks . . . . . . . . . . . . . . . . . 40

2.7 Future Work . . . . . . . . . . . . . . . . . . . 41

3 Rhodium Complexes $\quad 42$

3.1 Reactions of ${ }^{2-p y r} \mathrm{PNP}$ with

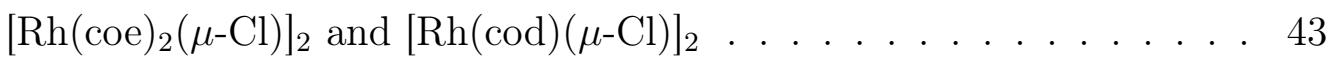

3.2 Reactions of ${ }^{2-\text { pyr }} \mathrm{PNP}$ with

$\left[\mathrm{Rh}(\text { acetone })_{2}(\mathrm{coe})_{2}\right] \mathrm{PF}_{6}$ and $\left[\mathrm{Rh}(\mathrm{coe})_{2}(\mu \text {-OTf })\right]_{2} \ldots \ldots . \ldots 46$

3.3 Concluding Remarks . . . . . . . . . . . . . . . . . . . . . 49

3.4 Future Work . . . . . . . . . . . . . . . . . . . . . 49

4 Iridium Complexes $\quad 51$

4.1 Reactions of ${ }^{2-p y r} \mathrm{PNP}$ with $\left[\operatorname{Ir}(\text { coe })_{2}(\mu-\mathrm{Cl})\right]_{2}$ and $\left[\operatorname{Ir}(\text { acetone })_{2}(\text { coe })_{2}\right] \quad . \quad 52$

$4.1 .1\left[\operatorname{Ir}(\mathrm{coe})_{2}(\mu-\mathrm{Cl})\right]_{2} \ldots \ldots \ldots \ldots . \ldots \ldots$

$4.1 .2\left[\operatorname{Ir}(\text { acetone })_{2}(\text { coe })_{2}\right] \mathrm{PF}_{6} \ldots \ldots \ldots \ldots$

4.2 Reactions of ${ }^{2-p y r} \mathrm{PNP}$ with $[\operatorname{Ir}(\operatorname{cod})(\mu-\mathrm{Cl})]_{2}$ and $[\operatorname{Ir}(\operatorname{cod})(\mu-\mathrm{OMe})]_{2} \ldots . \quad 54$

$4.2 .1[\operatorname{Ir}(\operatorname{cod})(\mu-\mathrm{Cl})]_{2} \ldots \ldots \ldots \ldots 6$

4.2.2 Comparison of ${ }^{2-\mathrm{pyr}} \mathrm{PNP}$ with ${ }^{\mathrm{Ph}} \mathrm{PNP}$. . . . . . . . . . . 60

$4.2 .3[\operatorname{Ir}(\operatorname{cod})(\mu-\mathrm{OMe})]_{2} \ldots \ldots \ldots \ldots$

4.3 Reactivity of $\left[\left({ }^{2-p y r} \mathrm{PNP}\right) \operatorname{Ir}(\operatorname{cod})\right] \mathrm{Cl} \ldots \ldots \ldots . \ldots . \ldots . \ldots$

4.3 .1 Ethene ...................... 65

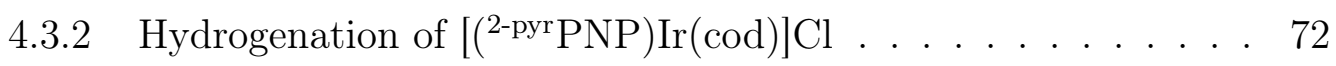

4.4 Concluding Remarks . . . . . . . . . . . . . . . 74

4.5 Future Work . . . . . . . . . . . . . . . . . . . 75

$\begin{array}{lll}5 \text { Catalysis } & 77\end{array}$

5.1 Terminal Alkenes and Alkynes . . . . . . . . . . . . . . . 78

5.2 Nitrile Hydration . . . . . . . . . . . . . . . . . . . 82

5.2 .1 Substrate Screening . . . . . . . . . . . . . 82

5.2 .2 Temperature and Catalyst Loading . . . . . . . . . . . 83

$5.2 .3 \quad\left[\left({ }^{2-\mathrm{pyr}} \mathrm{PNP}\right) \operatorname{Ir}(\mathrm{H})_{2} \mathrm{Cl}\right] \ldots \ldots \ldots$ 
$5.2 .4 \quad$ Pyridyl Groups . . . . . . . . . . . . . . . . . . . . 85

5.3 Concluding Remarks $\ldots \ldots \ldots \ldots$

5.4 Future Work . . . . . . . . . . . . . . . . . . . . . . . . . 87

6 Conclusion $\quad 88$

$\begin{array}{lll}7 & \text { Experimental } & 90\end{array}$

7.1 General Procedures . . . . . . . . . . . . . . . . . . . . . . . . . 90

7.2 Ligand Synthesis . . . . . . . . . . . . . . . . . . . 9 91

7.3 Metal Complexes . . . . . . . . . . . . . . . . . . 98

7.4 Catalysis . . . . . . . . . . . . . . . . . . 103

$\begin{array}{ll}\text { Appendix } & 105\end{array}$

8.1 Crystallographic Data for $\left[\left({ }^{2-p y r} \mathrm{PNP}\right) \operatorname{Ir}(\mathrm{cod})\right] \mathrm{Cl}(\mathbf{3 3}) \ldots \ldots \ldots \ldots$

8.2 NMR Spectra of Selected Compounds . . . . . . . . . . . . 107

$\begin{array}{ll}\text { Bibliography } & 120\end{array}$ 


\section{List of Figures}

1.1 Alkene hydration. . . . . . . . . . . . . . . . . . . . 2

1.2 Proposed catalytic cycles for styrene hydration. . . . . . . . . . 4

1.3 Catalytic cycle for anti-Markovnikov hydration. . . . . . . . 7

1.4 Interaction of water with a metal centre. . . . . . . . . . . 8

1.5 Insertion of an alkene into a metal-heteroatom or metal-H/C bond. . 9

1.6 Nucleophilic attack of water facilitated by a pyridyl phosphine. . . . 14

1.7 Nucleophilic attack facilitated by naphthyridine. . . . . . . . . . 15

1.8 Coordination modes of $\mathrm{Ph}_{2} \mathrm{P}(2$-pyr $) \ldots \ldots \ldots \ldots \ldots \ldots$

$1.9 \kappa^{2}-P, N$ chelate formation . . . . . . . . . . . . . . 16

1.10 Ruthenium-catalysed anti-Markovnikov hydration of terminal alkynes. 16

1.11 Activation of substrates by pendant boranes. . . . . . . . . . 18

2.1 Target ligands. . . . . . . . . . . . . . . . . . . . 20

2.2 Attempted synthesis of ${ }^{\mathrm{Cl}} \mathrm{PNP} \ldots \ldots \ldots \ldots \ldots . \ldots . \ldots 27$

$2.3{ }^{1} \mathrm{H}$ NMR of ${ }^{2-p y r} \mathrm{PNP}$ synthesised using $\mathrm{K} / \mathrm{NH}_{4} \mathrm{Cl} \ldots \ldots \ldots$

2.4 Reagents for quenching $\mathrm{K}(2$-pyridyl). . . . . . . . . . . . . 33

2.5 NMR spectrum of ${ }^{2-p y r} \mathrm{PNP}$ synthesis using lithium powder $/ \mathrm{NH}_{4} \mathrm{Cl} . \quad 34$

$2.6{ }^{1} \mathrm{H}$ NMR spectrum of ${ }^{2-\mathrm{pyr} P N P} \ldots \ldots \ldots \ldots$

2.7 Numbering scheme for the ${ }^{2-\text { pyr }}$ PNP ligand. . . . . . . . . . . . . 37

4.1 Reactivity of PNP and PPP ligands with iridium(I) cod complexes . . 55

4.2 Expansion of a ${ }^{1} \mathrm{H}-{ }^{15} \mathrm{~N}$ CIGAR-HMBC spectrum . . . . . . . . 57

4.3 X-ray structure and synthesis of $34 \ldots \ldots \ldots \ldots$

4.4 X-ray structure of $\left[\left({ }^{2-p y r} \mathrm{PNP}\right) \operatorname{Ir}(\mathrm{cod})\right] \mathrm{Cl} . \ldots \ldots \ldots \ldots$

4.5 Trigonal bipyramidal core of $33 \ldots \ldots \ldots$

4.6 Proposed mechanism for deuterium incorporation . . . . . . . . 62

4.7 Effect of deuteration on the ${ }^{13} \mathrm{C}$ NMR spectrum. . . . . . . . . . . 64

4.8 Change to ${ }^{31} \mathrm{P}\left\{{ }^{1} \mathrm{H}\right\}$ NMR spectrum. . . . . . . . . . . . . . . 66

$4.9{ }^{1} \mathrm{H}$ NMR spectra of 36 between +40 and $-70{ }^{\circ} \mathrm{C}$ in $\mathrm{CD}_{2} \mathrm{Cl}_{2} \ldots \ldots 6$

$4.10{ }^{1} \mathrm{H}$ NMR spectra of 36 between +50 and $-20{ }^{\circ} \mathrm{C}$ in 1:1 acetone- $\mathrm{d}_{6} / \mathrm{D}_{2} \mathrm{O} 69$

4.11 Change to pincer geometry when cod is displaced by ethene . . . . 70

$4.12{ }^{13} \mathrm{C}\left\{{ }^{1} \mathrm{H}\right\}$ NMR spectra of 36 at $+5{ }^{\circ} \mathrm{C}$ and $-40{ }^{\circ} \mathrm{C}$ in $\mathrm{CD}_{2} \mathrm{Cl}_{2} \ldots \ldots 71$ 
5.1 Hydration aided by the pincer backbone. . . . . . . . . . . . 78

5.2 Hydration aided by the pyridyl substituents. . . . . . . . . . . . . . 79

5.3 (E)-1,4-Bis(triisopropylsilyl)-1-buten-3-yne. . . . . . . . . . . . 80

5.4 Proposed catalytic cycle for (triisopropylsilyl)acetylene dimerisation. . 81

7.1 Numbering scheme for enyne product. . . . . . . . . . . . . . . . . 103

8.2 X-ray structure of $\left[\left({ }^{2-p y r} \mathrm{PNP}\right) \operatorname{Ir}(\operatorname{cod})\right] \mathrm{Cl}$ showing disordered pyridyl rings . . . . . . . . . . . . . . . . . . 105

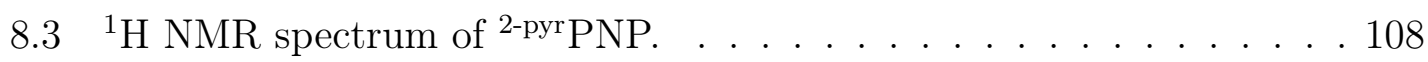

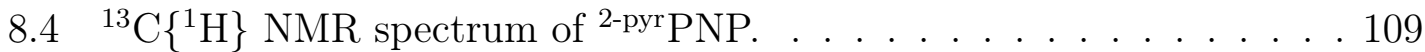

$8.5{ }^{1} \mathrm{H}$ NMR spectrum of ${ }^{2-p y r} \mathrm{PNP}^{-\mathrm{O}_{2}}$ (25). . . . . . . . . . . . . . 109

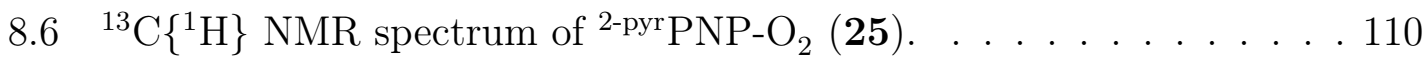

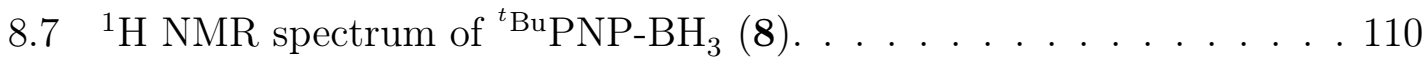

$8.8{ }^{13} \mathrm{C}\left\{{ }^{1} \mathrm{H}\right\}$ NMR spectrum of ${ }^{t}{ }^{\mathrm{Bu}} \mathrm{PNP} \mathrm{BH}_{3}(\mathbf{8}) \ldots \ldots \ldots . . \ldots 111$

8.9 The P-Vi groups have distinctive ${ }^{1} \mathrm{H}$ NMR signals. . . . . . . . . . . . . 111

$8.10{ }^{31} \mathrm{P}\left\{{ }^{1} \mathrm{H}\right\}$ NMR spectrum of ${ }^{2-p y r} \mathrm{PNP}+\left[\mathrm{Rh}(\text { acetone })_{2}(\text { coe })_{2}\right]$, at $=0.1$ s. 112

$8.11{ }^{31} \mathrm{P}\left\{{ }^{1} \mathrm{H}\right\} \mathrm{NMR}$ spectrum of ${ }^{2-\text { pyr }} \mathrm{PNP}+[\mathrm{Rh} \text { (acetone) })_{2}$ (coe) $\left.)_{2}\right]$, at $=0.025 \mathrm{~s} .112$

$8.12{ }^{31} \mathrm{P}\left\{{ }^{1} \mathrm{H}\right\}$ NMR spectrum of ${ }^{2-\text { pyr }} \mathrm{PNP}+\left[\mathrm{Rh}(\text { coe })_{2}(\mu-\mathrm{Cl})\right]_{2}$ in toluene. . 113

$8.13{ }^{31} \mathrm{P}\left\{{ }^{1} \mathrm{H}\right\}$ NMR spectrum of ${ }^{t} \mathrm{Bu} \mathrm{PNP}+\left[\mathrm{Rh}(\text { coe })_{2}(\mu-\mathrm{Cl})\right]_{2}$. . . . . . . 113

$8.14{ }^{31} \mathrm{P}\left\{{ }^{1} \mathrm{H}\right\}$ NMR spectrum of $\left[\left({ }^{t} \mathrm{Bu} \mathrm{PNP}\right) \mathrm{RhCl}\right]+\mathrm{AgBF}_{4}$. . . . . . . . . 114

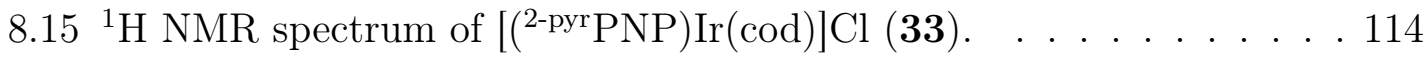

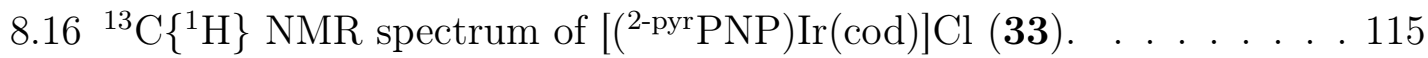

8.17 COSY spectrum of $\left[\left({ }^{2-p y r} \mathrm{PNP}\right) \operatorname{Ir}(\operatorname{cod})\right] \mathrm{Cl}(\mathbf{3 3}) \ldots \ldots . . \ldots 115$

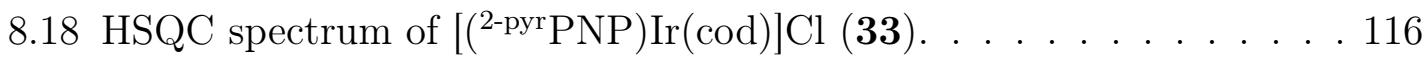

$8.19{ }^{1} \mathrm{H}$ NMR spectrum of ${ }^{2-\mathrm{pyr}} \mathrm{PNP}+[\operatorname{Ir}(\operatorname{cod})(\mu-\mathrm{OMe})]_{2}$. . . . . . . . . 117

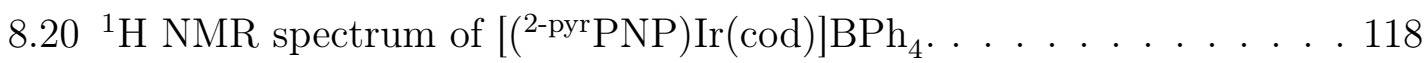

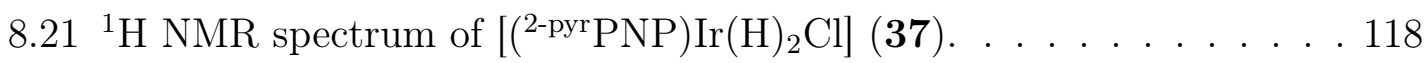

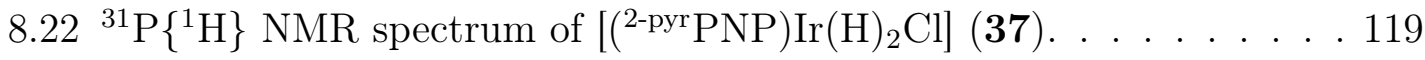




\section{List of Tables}

1.1 Effects of the pincer architecture on the properties of metal complexes. 12

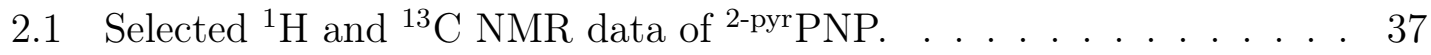

$2.2{ }^{31} \mathrm{P}\left\{{ }^{1} \mathrm{H}\right\}$ NMR data of phosphine selenides. . . . . . . . . . . . . . . 40

5.1 Substrate screening for nitrile hydration. . . . . . . . . . . . . 83

5.2 Reaction conditions. . . . . . . . . . . . . . . . . . . 84

5.3 Hydration of nitriles RCN using 5 mol\% [( $\left.\left.{ }^{2-p y r} \mathrm{PNP}\right) \operatorname{Ir}(\mathrm{H})_{2} \mathrm{Cl}\right] . \quad \ldots . \quad 84$

5.4 Comparison between ${ }^{2-p y r} \mathrm{PNP}$ and ${ }^{\mathrm{Ph}} \mathrm{PNP}$ ligands. . . . . . . . . . . . 85

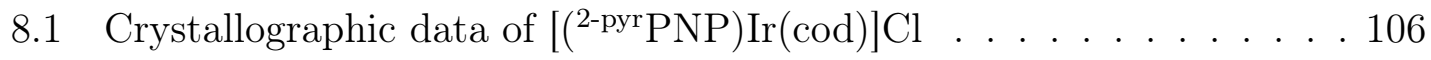




\section{List of Schemes}

1.1 Stoichiometric methods for the synthesis of primary alcohols. . . . . . 3

1.2 Synthesis of primary alcohols from styrene derivatives using tandem catalysis. . . . . . . . . . . . . . . . . . 3

1.3 Synthesis of primary and secondary alcohols from alkynes. . . . . . . 5

1.4 Hydrolysis of nitriles. . . . . . . . . . . . . . . . . . 6

1.5 Use of a hydration catalyst in the synthesis of a psymberin analogue. 6

1.6 Oxidative addition of water to an iridium complex. . . . . . . . . . 9

1.7 Addition of water to an iridium complex to form a hydrido-hydroxo complex. . . . . . . . . . . . . . . . . 9 9

1.8 Reaction of a platinum methoxide complex with $\mathrm{CF}_{2} \mathrm{CF}_{2} \ldots \ldots$

1.9 Ligand-induced reductive elimination of ethanol. . . . . . . . . . . . . 11

1.10 Effect of the pincer backbone on oxidative addition. . . . . . . . . . . 13

1.11 Catalytic water splitting aided by backbone dearomatisation. . . . . . 17

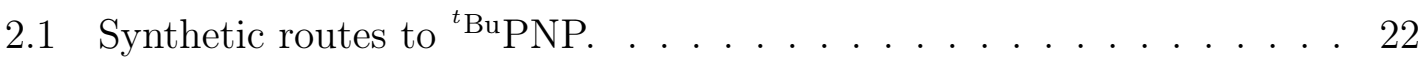

2.2 Synthesis of ${ }^{t}{ }^{\mathrm{Bu}} \mathrm{PNP}\left(\mathrm{BH}_{3}\right) \ldots \ldots \ldots \ldots \ldots \ldots \ldots$

2.3 Lithium-bromine exchange. . . . . . . . . . . . . . . . . . . 24

2.4 Deprotection of ${ }^{t} \mathrm{Bu} P N P\left(\mathrm{BH}_{3}\right) \ldots \ldots \ldots \ldots . \ldots \ldots 24$

2.5 Routes to ${ }^{\mathrm{Vi}} \mathrm{PNP}$ based on literature syntheses of ${ }^{t} \mathrm{Bu} \mathrm{PNP} . \quad \ldots . . .25$

2.6 Synthesis of $\mathrm{CF}_{3} \mathrm{PCP}$ by Roddick et al. . . . . . . . . . . . . . . . 26

2.7 Phosphonite synthesis. . . . . . . . . . . . . . . . . . . . . 27

2.8 Synthesis of $\mathrm{Vi}^{\mathrm{P}} \mathrm{PNP} \ldots \ldots \ldots$. . . . . . . . . . . . . . . . . . . 28

2.9 Attempted synthesis of 22. . . . . . . . . . . . . . . . . . 29

2.10 Original route to 2 -pyridyl-substituted phosphines. . . . . . . . . . . 30

2.11 Ligand coupling to form 2,2'-bipyridine. . . . . . . . . . . . . 35

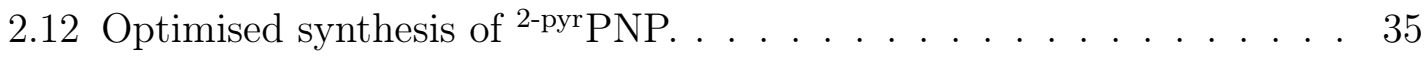

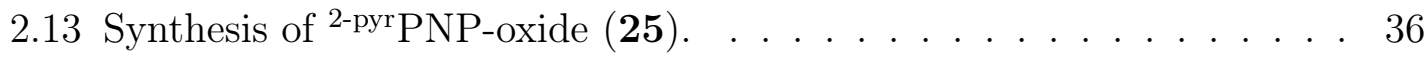

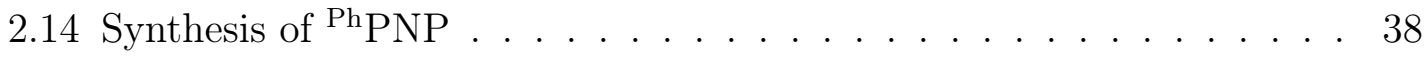

2.15 Synthesis of phosphine selenides. . . . . . . . . . . . . . 40

3.1 Possible chloroform-soluble rhodium(III) complexes. . . . . . . . . . . 43

3.2 Possible $\mathrm{D}_{2} \mathrm{O}$-soluble rhodium complexes. . . . . . . . . . . . . . 45 
3.3 Reaction of ${ }^{2-p y r} \mathrm{PNP}$ with $\left[\mathrm{Rh}(\mathrm{coe})_{2}(\mu-\mathrm{Cl})\right]_{2}$ in toluene. . . . . . . . . 45

3.4 Reactions of PNP ligands with rhodium(I) cyclooctene complexes. . . 47

3.5 Attempted synthesis of $[(\mathrm{PNP}) \mathrm{Rh}(\mathrm{OTf})] \ldots \ldots \ldots$. . . . . . . . . 48

3.6 Synthesis of a known rhodium(II) complex. . . . . . . . . . . . . . . . 48

4.1 Vinylic $\mathrm{C}-\mathrm{H}$ activation . . . . . . . . . . . . . . . . . 52

4.2 Reaction of $\left[\operatorname{Ir}(\text { acetone })_{2}(\text { coe })_{2}\right] \mathrm{PF}_{6}$ with ${ }^{2-p y r} \mathrm{PNP} \ldots \ldots \ldots \ldots$

4.3 Synthesis of 35. . . . . . . . . . . . . . . . . . . . 61

4.4 Synthesis of bis(ethene) complex 36. . . . . . . . . . 65

4.5 Synthesis of 37. . . . . . . . . . . . . . . . 73

5.1 Hydration of benzonitrile . . . . . . . . . . . . . . . . 82 


\section{Pincer Ligand Terminology and Glossary}

This thesis employs naming conventions common to the pincer ligand literature:

PCCCP, PONOP, PNNNP, etc. Represents ligands based on the 1,3-phenylene or 2,6-pyridyl backbone. The donor atoms of the ligand are denoted by the first, third, and fifth letters. The linkages between the donor groups (the "arms" of the ligand) are denoted by the second and fourth letters.

PCP, PNP A shortened version of the PCCCP and PCNCP naming convention described above.

[(pincer) $\left.\mathbf{M L}_{n}\right]$ Literature convention for presenting pincer complexes. For example, $\left[\left({ }^{2-p y r} \mathrm{PNP}\right) \operatorname{Ir}(\operatorname{cod})\right] \mathrm{Cl}$ could be named:

$\left[\operatorname{Ir}\left(\eta^{4}-1,5\right.\right.$-cyclooctadiene $\left.)\left(1,3-\left[(2-\text { pyr }){ }_{2} \mathrm{PCH}_{2}\right]_{2} \mathrm{C}_{5} \mathrm{H}_{3} \mathrm{~N}-\kappa^{2} P \kappa N\right)\right] \mathrm{Cl}$.

BArF tetrakis[3,5-bis(trifluoromethyl)phenyl]borate

CIGAR Constant time inverse-detection gradient accordion rescaled coe cyclooctene

cod 1,5-cyclooctadiene

COSY correlation spectroscopy

CSD Cambridge Structural Database

Cp cylopentadienyl

DFT density functional theory

dppf 1,1'-bis(diphenylphosphino)ferrocene

ESI electrospray ionisation

GC-MS gas chromatography-mass spectrometry

HMBC heteronuclear multiple bond correlation

HSQC heteronuclear single quantum coherence

IR infrared spectroscopy

PNP Diphosphine ligand with a 2,6-dimethylpyridine backbone

pyr pyridine

THF tetrahydrofuran

TMS trimethylsilyl

TOF turnover frequency (mol product/mol catalyst $\times$ h)

2-pyr 2-pyridyl, $\mathrm{C}_{5} \mathrm{H}_{4} \mathrm{~N}$

9-BBN 9-borabicyclo[3.3.1]nonane 


\section{Chapter 1}

\section{Introduction}

In a time where the effects of anthropogenic climate change are becoming ever clearer, the field of chemistry will be required to assume a central role in developing more efficient and environmentally friendly ways of using our natural resources. Chemistry today still relies on raw materials derived from the petrochemical industry. However, it is clear that chemicals derived from renewable sources will be required to replace these.

The use of water as a reagent or solvent is a particularly "green" goal. Water is extremely abundant, cheap, and has even been shown to accelerate the rates of certain organic transformations. ${ }^{1}$ Although many organic compounds are not miscible with water, a number of possible solutions to this problem have been developed. These include phase transfer catalysts, microemulsion reactions, "on-water" chemistry, and solvent mixtures. ${ }^{2}$

Water can be used to synthesise oxygen-containing functional groups. These find use in a huge variety of applications. For example, simple alcohols are valuable starting materials in the plastics industry, ketones and aldehydes are important in polymer synthesis and perfumes, and amides are common in drug molecules. These four functional groups can be accessed from the stoichiometric reaction of alkenes, alkynes, and nitriles with water. However, water does not react quickly with these unsaturated moieties unless a suitable catalyst is present due to repulsion between the electron-rich multiple bond and the oxygen atom's non-bonding electrons. It can also be difficult to achieve the desired amount of control over hydration reactions. In fact, regioselective addition of heteroatom nucleophiles (principally water and ammonia) to unactivated alkenes was named as one of ten "challenges for catalysis" in 1993. ${ }^{3}$ Furthermore, the ability to regio- and stereo-selectively hydrate alkenes under 
mild conditions is "extremely desirable" 4 and is a reaction "of utmost importance" 5 as it would represent the first atom-economic route to primary alcohols. Amide synthesis has also been described as one of the most troublesome transformations currently required by the pharmaceutical industry. ${ }^{6}$

The importance of these reactions is evident from the recent growth in the field of catalytic hydrofunctionalisation chemistry, particularly that concerned with the hydration of unsaturated substrates by late transition metal catalysts. ${ }^{5,7}$ This chapter presents an overview of late transition metal-catalysed alkyne, alkene, and nitrile hydration chemistry, with a specific focus on the interaction and reactivity of water with late transition metals. It describes the problems of, and potential solutions to, using water as a reactant in coordination chemistry and catalysis.

\section{$1.1 \quad$ Alkenes}

Addition reactions are the most widely used method for the derivatisation of carboncarbon double bonds. ${ }^{5}$ These reactions provide a direct route to alcohols and show complete atom and redox economy. ${ }^{8,9}$ In the first step of a traditional acid-catalysed alkene hydration reaction, a carbocation intermediate is formed (Figure 1.1). The stability of this intermediate controls the overall regioselectivity of the reaction; unsymmetrical substrates lead to the thermodynamically and kinetically favoured secondary alcohols.

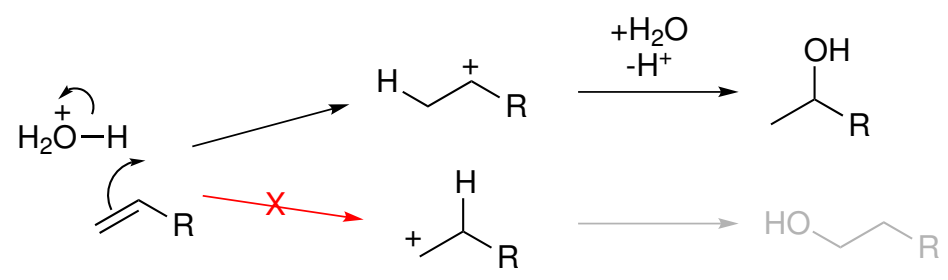

Figure 1.1 Regioselectivity in the hydration of a terminal alkene.

Vladimir Markovnikov summarised this selectivity in his eponymous rule, which can be generalised to the addition of other polar compounds across unsaturated systems:

"When a hydrocarbon of unsymmetrical structure combines with a hydrogen haloacid, the halogen adds itself to the less hydrogenated carbon atom, that is, to the carbon atom which is more under the influence of other carbon atoms." ${ }^{5,10}$

Industrially, the direct addition of water across carbon-carbon double bonds is carried out over solid supports such as zeolites or with Brønsted acid catalysts. ${ }^{11,12}$ However, 
it is only secondary alcohols that can be produced in this way. Primary alcohols, although more valuable, can only be synthesised using stoichiometric methods (Scheme 1.1). ${ }^{13}$ These are wasteful and cannot be carried out in water.

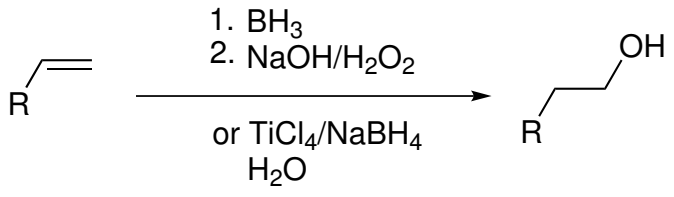

Scheme 1.1 Stoichiometric methods for the synthesis of primary alcohols.

There exists very few examples of primary alkenes having undergone catalytic anti-Markovnikov heterofunctionalisation, let alone anti-Markovnikov hydration. ${ }^{7,12}$ However, the allure of a 100\% atom- and redox-economic hydration reaction remains a cause for fundamental research in organometallic chemistry.

Four years ago, the Grubbs group published an elegant application of tandem catalysis that, using both Wacker oxidation chemistry and ruthenium-catalysed transfer hydrogenation, could selectively oxidise styrene derivatives to primary alcohols (Scheme 1.2). ${ }^{12}$ This is the only example of the successful catalytic antiMarkovnikov hydration of unactivated alkenes.

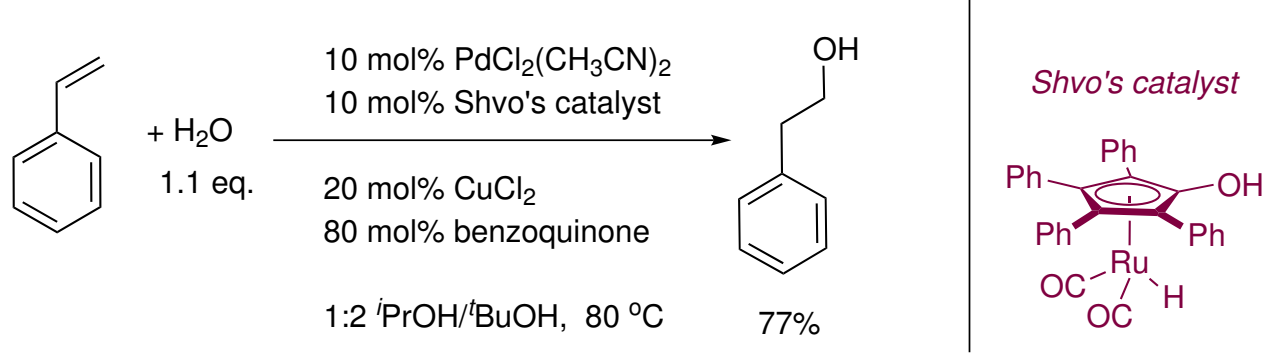

Scheme 1.2 Synthesis of primary alcohols from styrene derivatives using tandem catalysis.

The two catalysts acted in isolation: hydride transfer to Shvo's catalyst was independent of the oxidation cycle, and stoichiometric amounts of benzoquinone $/ \mathrm{CuCl}_{2}$ were required to oxidise the palladium (Figure 1.2). Linear selectivity is usually disfavoured by Wacker chemistry. However, by using bulky tert-butanol as a cosolvent, selectivities greater than 20:1 in favour of the primary alcohol were obtained. Tests using aliphatic substrates showed that styrene was required for high selectivity. Styrene binds $\eta^{4}$ to palladium, which results in nucleophilic attack by tert-butanol occurring at the terminal carbon. Water is required to hydrolyse the vinyl ether, which then joins the reduction cycle. 


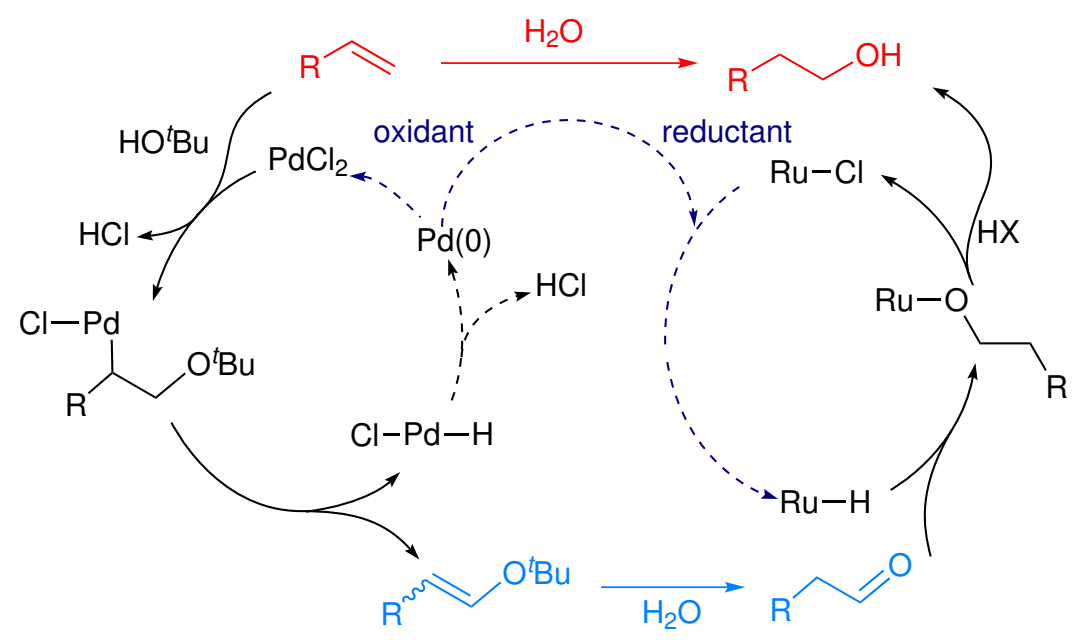

Figure 1.2 The catalytic cycles thought to be responsible for the high anti-Markovnikov selectivity in the hydration of styrene (the overall reaction is shown in red, uncoupled steps in dark blue). ${ }^{12}$

\section{$1.2 \quad$ Alkynes}

A number of late transition metal complexes catalyse the hydration of terminal alkynes. This reaction has also been reported under aqueous conditions. ${ }^{5,14,15}$ Ketones are usually produced as they are the Markovnikov product, but aldehyde-selective catalysts have also been reported. The first catalyst giving anti-Markovnikov selectivity was reported in 1998 by Tokunaga and Wakatsuki. ${ }^{16}$ Prior to this report, aldehydes could only be obtained from alkynes by stoichiometric methods. A large number of newer catalysts are based on their original catalyst, $\left[\mathrm{RuCl}_{2}\left(\mathrm{C}_{6} \mathrm{H}_{6}\right) \mathrm{PPh}_{2}\left(\mathrm{C}_{6} \mathrm{~F}_{5}\right)\right]$. These include those based on $\left[\mathrm{RuCp}\left(\mathrm{PR}_{3}\right)_{2}\right] \mathrm{X}$ (where $\mathrm{X}$ is a weakly-coordinating counterion and $\mathrm{R}$ is a substituted phenyl group). ${ }^{17}$

A significant report by Watanabe et al. describes a water-soluble iridium complex that produces aldehydes or ketones from phenylacetylene, depending on the $\mathrm{pH}$ of the reaction mixture. ${ }^{14}$ Many of the intermediates in the catalytic cycle were isolated and a mechanism proposed based on isotopic labelling studies. Unlike ruthenium half-sandwich complexes, whose anti-Markovnikov selectivity often occurs through an alkyne/vinylidene isomerisation pathway, ${ }^{18}$ selectivity depended on the reaction that occurred at the proposed $\eta^{2}$-alkyne intermediate. When this was deprotonated, an alkynyl complex formed, which led to phenylacetaldehyde as the product. If it was attacked by $\mathrm{OH}^{-}$, a ketonyl complex formed; this was the precursor to acetophenone. ${ }^{14}$

Herzon and Li combined alkyne hydration with a hydrogenation step to synthesise alcohols from reduction of the carbonyl group. ${ }^{19}$ This was carried out using transfer hydrogenation. Here, the source of hydrogen is a small organic molecule such as 
isopropanol rather than dihydrogen. This report is very significant, as by using two different hydration catalysts, Herzon and Li developed a system that could produce both primary and secondary alcohols (Scheme 1.3). When 6-tert-butyl-2diphenylphosphinylpyridine ligands were used (Grotjahn's catalyst, $\mathbf{1}^{18}$ ), the resulting catalyst was extremely selective for the aldehyde product. This was reduced to give the primary alcohol. ${ }^{19}$ The use of a gold(I) N-heterocyclic carbene complex $\left(\mathbf{2}\right.$, known to be successful in gold catalysis $\left.{ }^{20}\right)$ provided a high-yielding route to secondary alcohols via a ketone intermediate. ${ }^{19}$

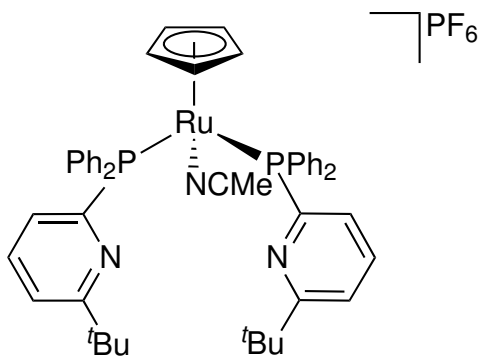

1
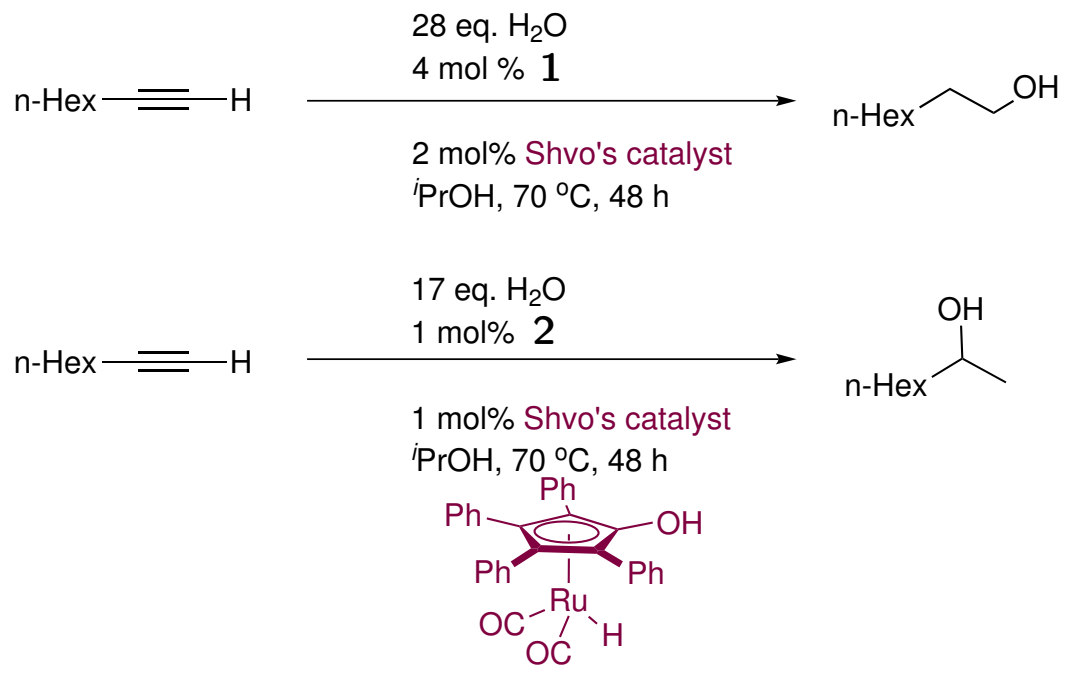

Scheme 1.3 Synthesis of primary and secondary alcohols from alkynes using tandem catalysis. ${ }^{19}$

\section{$1.3 \quad$ Nitriles}

Nitrile hydration is the most atom-econonical route to primary amides. However, when this reaction is carried out under acid- or base-promoted conditions, it often results in product mixtures due to over-hydrolysis to the corresponding carboxylic acid. This occurs because the second step shown in Scheme 1.4 is faster than the first. $^{21}$

Transition metal catalysts have been developed to overcome this chemoselectivity problem. These are often able to hydrate nitriles under much milder conditions 


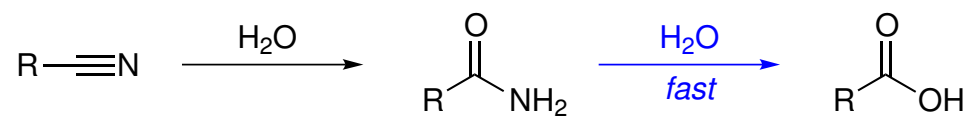

Scheme 1.4 Hydrolysis of nitriles.

(including ambient temperature) and display dramatically improved tolerance towards sensitive functional groups.

Nitriles can coordinate to transition metals through the lone pair of electrons on nitrogen - a mode of reactivity that is not available to alkynes or alkenes. As well as the potential for activation via $\eta^{2}$ coordination, $\kappa^{1} N$-coordination activates nitriles towards nucleophilic attack. This is the dominant pathway in late transition metalcatalysed hydration and has been exploited in "bifunctional" hydration catalysts (see section 1.6).

Particularly relevant are the numerous reports of nitrile hydration catalysts that function under aqueous conditions. ${ }^{22}$ These include platinum, nickel, and ruthenium complexes. Ruthenium catalysts are particularly active. A feature of the most active catalysts is that their ligands introduce heteroatoms near the active site. These activate water towards nucleophilic attack on the nitrile. ${ }^{23,24} \mathrm{~A}$ number of rhodium complexes have shown high activity. The Saito and Bera groups have reported extremely active rhodium catalysts that function in aqueous isopropanol. ${ }^{25,26}$ Only three iridium complexes have been reported to carry out this transformation in any solvent.

The platinum(II) complex reported by Parkins in 1995 has been used successfully as a catalyst in the syntheses of more complex substrates. ${ }^{27}$ For example, De Brabander et al. relied on it to hydrate a nitrile group in their synthesis of cytotoxic psymberin analogues (Scheme 1.5). ${ }^{28}$
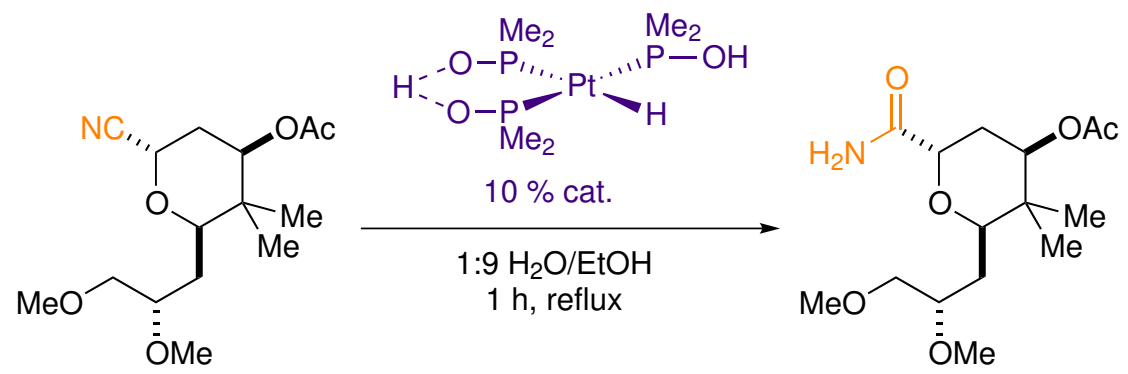

Scheme 1.5 Use of a hydration catalyst in the synthesis of a psymberin analogue. 


\subsection{Elementary Steps}

Homogeneous catalysis represents the major contribution of organometallic chemistry to chemical synthesis. Alkene metathesis, asymmetric hydrogenation, and crosscoupling reactions are just a few of the catalytic reactions that have become an indispensable part of the synthetic chemist's armamentarium. Metal catalysts provide a reaction coordinate that considerably reduces the energy required to affect a chemical transformation.

Using late transition metals, it is possible to catalyse the formation of carbonheteroatom bonds through reactions such as hydrosilylation $(\mathrm{C}-\mathrm{Si})$, hydroamination $(\mathrm{C}-\mathrm{N})$, and hydrophosphination $(\mathrm{C}-\mathrm{P}) .{ }^{29}$ The synthesis of $\mathrm{C}-\mathrm{O}$ bonds using late transition metal catalysts is much less developed, particularly concerning bonds to $\mathrm{sp}^{3}$ carbons or those where the oxygen atom is derived from water.

The hydration of alkenes using a single catalytic cycle has never been observed. Thus, an investigation into this highly desirable reaction is a good lens through which to analyse the reactivity of water with late transition metal complexes.

A plausible catalytic cycle for anti-Markovnikov hydration can be constructed by applying the typical mechanistic steps observed for these reactions (Figure 1.3). The regioselectivity shown stems from traditional reactions in organometallic chemistry, but the cycle shown is not necessarily limited to it. ${ }^{30}$

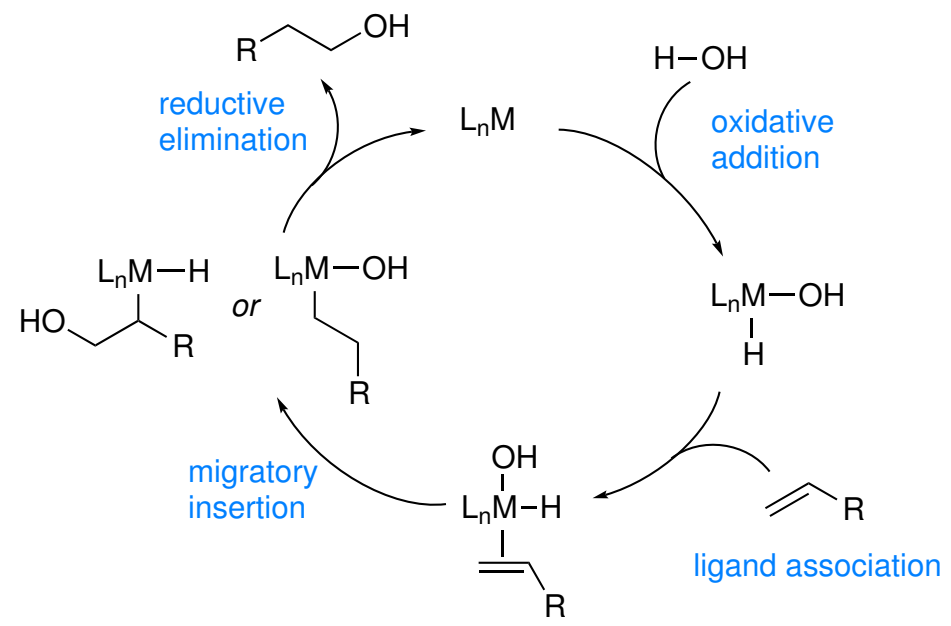

Figure 1.3 Catalytic cycle for the anti-Markovnikov addition of water to an unactivated alkene.

Although Figure 1.3 involves common elementary steps, the cycle presented has never been observed. ${ }^{7}$ In fact, it is even rare to find reports of these steps occurring in isolation; described below are some of the reasons for this. 


\subsubsection{Oxidative Addition}

Oxidative addition is a very important elementary step, as it is often responsible for activating the substrate. By forming two new metal-ligand bonds, further metalmediated reactions are possible. In the case of water, this requires the cleavage of an $\mathrm{O}-\mathrm{H}$ bond. However, water is a good Lewis base and Lewis acid-Lewis base interactions may outcompete cleavage reactions. ${ }^{31}$ In fact, the interaction of water with a metal centre can occur in a number of ways (Figure 1.4). Coordinatively saturated 18 electron complexes are often protonated or undergo hydrogen bonding interactions. ${ }^{31}$

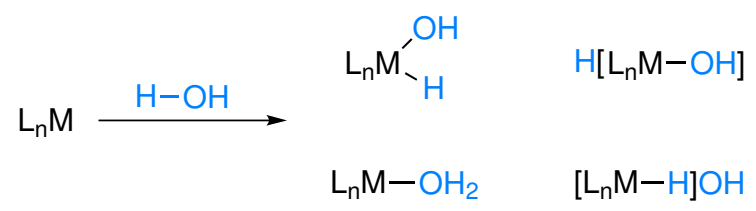

Figure 1.4 Interaction of water with a metal centre. ${ }^{31}$

While oxidative addition of water to a transition metal would fulfil the first step in Figure 1.3, this reaction is rare compared to those involving $\mathrm{C}-\mathrm{X}$ and $\mathrm{C}-\mathrm{H}$ bonds. ${ }^{30}$ Few complexes resulting from the direct oxidative addition of water have been isolated, although they have been implicated in a number of reactions. ${ }^{4,32}$ Even metal alkoxides formed from the oxidative addition of alcohols are rare; these complexes are prone to decomposition via $\beta$-hydride elimination. ${ }^{4,30}$ Trapping the hydride and hydroxo ligands after an oxidative addition reaction is also difficult. ${ }^{29}$ Rearrangement of the extremely nucleophilic hydroxo ligands to form bridging species, as well as rapid reactions of the hydroxo ligands, are common reactions that can mask the oxidative addition process. ${ }^{29}$

The 1970s saw the first reports of oxidative addition of water, first to rhodium(I) and then to platinum(0). ${ }^{33,34}$ In 1979, Otsuka and co-workers demonstrated the formation of a stable hydrido-hydroxo complex. This was generated by oxidative addition of water to the two-coordinate complex generated by dissociation of a $\mathrm{P}\left({ }^{i} \mathrm{Pr}\right)_{3}$ ligand from $\left[\mathrm{Pt}\left(\mathrm{P}\left({ }^{i} \mathrm{Pr}\right)_{3}\right)_{3}\right]$.

The formation of Ir- $\mathrm{O}$ bonds through oxidative addition pathways has been reported in a number of studies. It is considered by a number of authors to be reactivity that is well-established. ${ }^{35-38}$ Milstein and co-workers studied the mechanisms of methanol and water addition to $\left[\mathrm{IrCl}\left(\mathrm{PMe}_{3}\right)_{3}\right](\mathbf{3}$, Scheme 1.6). They found that oxidative addition of water occurred at room temperature in THF through nucleophilic attack by the metal on the hydroxyl proton. ${ }^{32}$ The chlorido ligand was important as it stabilised the transition state through hydrogen bonding to the hydroxy proton. ${ }^{32}$ 


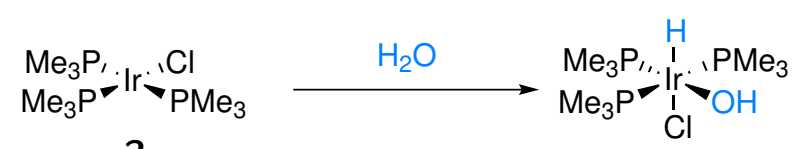

3

Scheme 1.6 Addition of water to the 16 electron complex 3 formed the kinetic hydrido-hydroxo product. ${ }^{32}$

Oxidative addition of water to the electron-rich iridium pincer complex 4 , shown in Scheme 1.7, was facile. This complex is active in the transfer dehydrogenation of cyclooctane but is unable to hydroxylate it. ${ }^{39}$
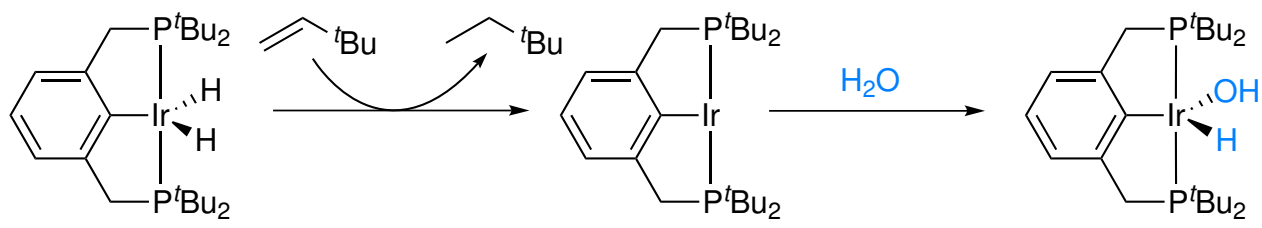

4

Scheme 1.7 Addition of water to the 14 electron complex 4 formed a 16 electron hydrido-hydroxo complex. ${ }^{39}$

\subsubsection{Migration}

Insertion of alkenes into $\mathrm{M}-\mathrm{H} / \mathrm{C}$ bonds is a common reaction, but it is only recently that evidence for insertion into $\mathrm{M}-\mathrm{O}$ (and $\mathrm{M}-\mathrm{N}$ ) bonds has been discovered. ${ }^{40,41}$ Most hydroxo, alkoxo, and amido complexes form dimeric/oligomeric species by bridging through $\mathrm{O}$ or $\mathrm{N}$, preventing alkenes from binding. ${ }^{41}$

Two products can arise from the insertion of alkenes into metal-element bonds. This depends on the electronic properties of the system, with the majority of reactions resulting in 1,2-insertion. ${ }^{40}$ Regioselectivity is similar for insertion into metal-heteroatom bonds (Figure 1.5). ${ }^{42}$ In the catalytic cycle shown in Figure 1.3, the alkene could insert into either the $\mathrm{M}-\mathrm{H}$ or the $\mathrm{M}-\mathrm{O}$ bond; however, it is likely that insertion into the $\mathrm{M}-\mathrm{H}$ bond is the only pathway to the anti-Markovnikov product. $^{42}$

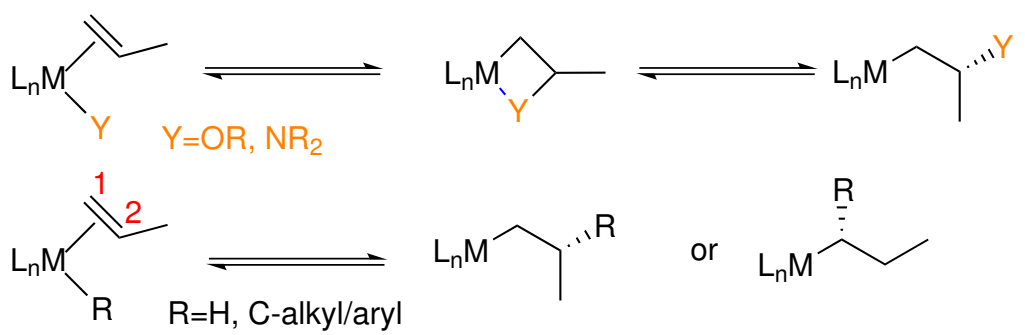

Figure 1.5 Insertion of an alkene into a metal-heteroatom or metal-H/C bond.

There are few reports of alkene insertion into $\mathrm{M}-\mathrm{O}$ bonds, as much of the research on 
catalytic heterofunctionalisation has focussed on insertion into M-N bonds. ${ }^{41} 30$ Even this research is recent; for example, it was only in 2010 that alkene insertion into $\mathrm{Pd}-\mathrm{N}$ bonds was reported. ${ }^{43}$ Stoichiometric reactions with iridium, platinum, and rhodium amido complexes have been demonstrated previously. ${ }^{35,41}$ In 1985, Bryndza found that tetrafluoroethene could insert into the platinum-oxygen bonds of methoxy ligands while ethene could not (Scheme 1.8). ${ }^{44}$ The Hartwig group has reported two of only four examples where there exists direct evidence for an alkene having inserted into a $\mathrm{M}-\mathrm{O}$ bond. In 2006, intramolecular insertion of an alkene into an alkoxo rhodium complex was observed. ${ }^{45}$ In 2013 Hartwig and co-workers reported the intermolecular insertion of an alkene into a $\mathrm{M}-\mathrm{O}$ bond in the presence of a $\mathrm{M}-\mathrm{H}$ bond. ${ }^{35}$

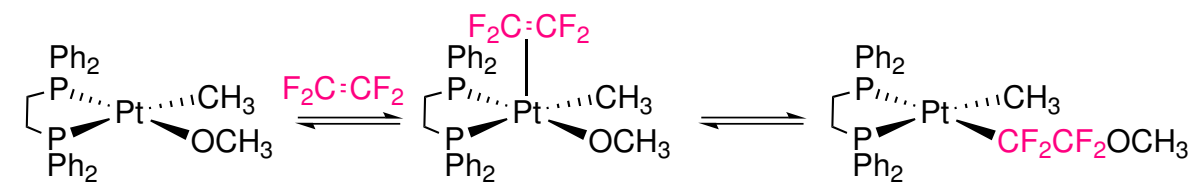

Scheme 1.8 Reaction of a platinum methoxide complex with $\mathrm{CF}_{2} \mathrm{CF}_{2} \cdot{ }^{44}$

\subsubsection{Reductive Elimination}

Reductive elimination is the reverse of oxidative addition and the final step in many catalytic reactions. This reaction forms a new bond, releasing the product from the metal in the process.

If hydration is to occur in a single catalytic process, reductive elimination must form either a $\mathrm{C}-\mathrm{H}$ or $\mathrm{C}-\mathrm{OH}$ bond, depending on the direction of migratory insertion. Reductive elimination involving hydride ligands is very common. ${ }^{46}$ Well-established processes such as hydroformylation rely on this reaction to form $\mathrm{C}-\mathrm{H}$ bonds. ${ }^{40}$ In contrast, coupling to form carbon-heteroatom bonds is much rarer. $\beta$-hydride elimination is often a major side reaction of heteroatom complexes able to undergo reductive elimination. ${ }^{47}$ There are no examples of $\mathrm{C}-\mathrm{OH}$ bond formation by direct reductive elimination.

Polarisability, electronegativity, and nucleophilicity are important factors in determining the rates of reductive elimination for reactions involving heteroatoms. ${ }^{46}$ These all disfavour reductive elimination reactions involving oxygen and are consistent with experimental evidence. For example, palladium-alkoxo complexes have been found to undergo reductive elimination less readily than analogous amido complexes, and formation of heterofunctionalised products containing oxygen, such as alkyl ethers, is rare. ${ }^{46}$ Reductive elimination from iridium to form $\mathrm{O}-\mathrm{H}$ bonds was observed by Glueck et al. However, the reaction shown in Scheme 1.9 did not go through a 
concerted pathway. ${ }^{48}$

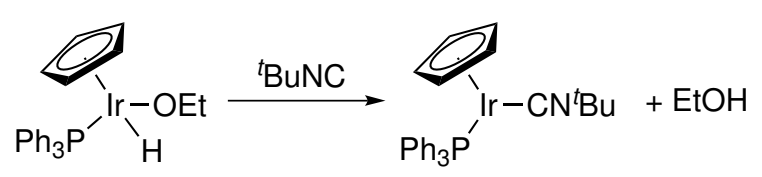

Scheme 1.9 Ligand-induced reductive elimination of ethanol was observed when a number of ligands $\left({ }^{t} \mathrm{BuNC}\right.$, ethene, $\left.\mathrm{CO}\right)$ were added to the iridium complex.

\subsection{Catalyst Design}

It is clear that the catalytic cycle outlined in Figure 1.3 is unlikely to occur without clever catalyst design. While coordinated alkenes are activated towards nucleophilic attack, they can be displaced by Lewis bases if the metal is too electron-poor. ${ }^{49}$ Thus, disfavouring Lewis acid-Lewis base adduct formation will require an electron-rich metal, which will in turn disfavour reductive elimination. The insertion of alkenes into the $\mathrm{M}-\mathrm{H}$ bonds generated by the oxidative addition of oxygen-containing compounds is also rare. ${ }^{7}$

It is clear then that a large number of variables must be considered when designing a catalyst. The ideal catalyst generates products efficiently, is recyclable, and functions under mild conditions with low loading. It is highly desirable from an environmental perspective for the complex to either show controlled reactivity with water or to be compatible with it.

Key to catalyst design is having an understanding of the coordination chemistry of the complex. This is controlled by the shape of the site to which the reactants bind and the electronic properties of the metal. Late transition metals are electron-rich and have a number of accessible oxidation states, making them well-suited to applications in catalysis. ${ }^{40}$

\subsubsection{Ligands}

Ancillary ligands are used to stabilise the metal and to tune its steric and electronic properties. Certain ligands are also able to increase the rate of substitution of mutually trans ligands. These ligands are usually strong $\sigma$-donors or strong $\pi$ acceptors. ${ }^{40}$ Phosphorus donor atoms are well suited to the stabilisation of late transition metal complexes. They are soft, good $\sigma$-donors and can accept electron density through a $\pi$-symmetry interaction. By modifying the groups attached to 
phosphorus, the magnitude of these interactions can be changed; this will affect the electronic properties of the metal centre.

The choice between monodentate and chelating ligands must be carefully considered. Chelating ligands exert a greater ordering effect on the coordination sphere than monodentate ligands. Investigations into the coordination chemistry of transition metal complexes containing tridentate pincer ligands support their use as catalysts for organic reactions. These strongly binding ligands have tuneable properties, and this is seen as a major advantage in reactions involving the activation of strong bonds. ${ }^{36,50}$ A number of pincer complexes have shown promise in water and ammonia activation. ${ }^{51,52}$ The effects of modifying parts of the pincer ligand backbone, such as the central donor atom $(\mathrm{X})$, on the properties of the resulting metal complex are summarised in Table 1.1.

Table 1.1 Effects of pincer architecture on the steric, electronic, and physical properties of the metal complex. Adapted from the 2011 review by Choi and co-workers. ${ }^{53}$

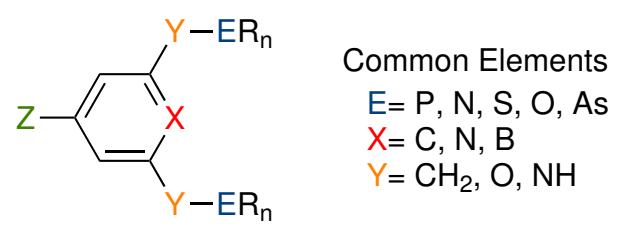

\begin{tabular}{ll}
\hline Group & Effect(s) of Variation \\
\hline $\mathrm{ER}_{\mathrm{n}}$ & Electron density, steric control \\
$\mathrm{Y}$ & Electron density, indirect steric control, cooperativity \\
$\mathrm{X}$ & Electronic effects, trans-influence \\
$\mathrm{Z}$ & Electron density (remote control), solubility \\
\hline
\end{tabular}

An elegant report by Hartwig and co-workers demonstrates the importance of the ligand backbone in determining the properties of the metal centre. ${ }^{36}$ The reactivities of two iridium complexes bearing different pincer ligands with ammonia were compared. The ligand in Scheme 1.10A was not electron-rich enough to destabilise the ammonia coordination complex. Considering the Lewis basicity of ammonia, it is unsurprising that it also difficult to form stable amido hydride complexes. ${ }^{36,54,55}$ However, by using a more electron-rich alkyl backbone, the product of oxidative addition was stable enough to be characterised by NMR spectroscopy and X-ray diffraction. ${ }^{36}$ 
A

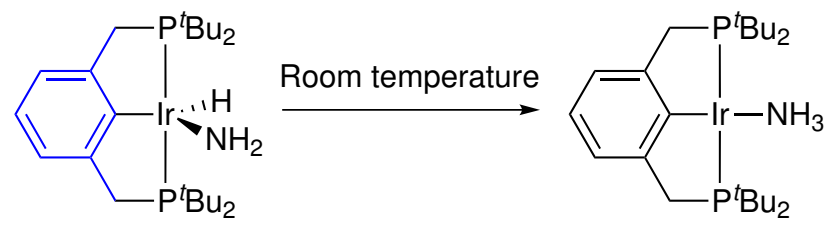

B

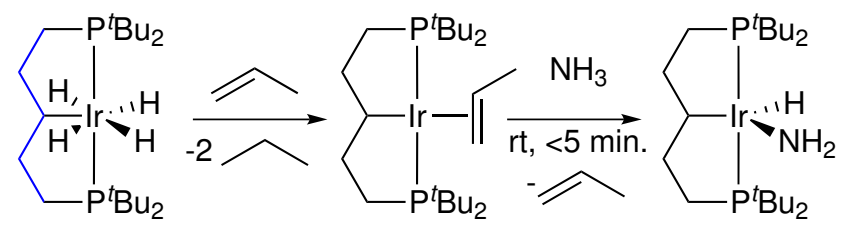

Scheme 1.10 Modifying the electronic properties of the metal centre by changing the pincer backbone resulted in the formation of a stable amido hydride complex.

The properties of the $\mathrm{R}$ groups affect the shape of the active site and the stability of the metal complex. These, along with position Z, can also be modified to confer water solubility on the metal complex. ${ }^{56}$ Pincer ligands favour meridional coordination and, due to the coplanarity of the metal with at least three donor atoms in this geometry, the transfer of steric bulk to the metal centre is made more efficient than for flexible ligands.

\subsection{Cooperative Catalysis}

The difficulties encountered in reacting water with transition metals may be minimised by decoupling the elementary steps required for this reaction from the metal centre. This strategy is inspired by processes that occur in biological systems; cooperativity between metal sites and surrounding amino acid residues is essential in the activation of small molecules by biological systems. For example, the active site of the enzyme that converts water into $\mathrm{O}_{2}$, photosystem II, involves a cooperative $\mathrm{Mn}_{4} \mathrm{O}_{4} \mathrm{Ca}$ cluster. ${ }^{57}$ Enzymes orient substrates in the active site through hydrogen bonding, and amino acid residues participate in the reaction. ${ }^{58,59}$ It is only recently that these interactions have begun to be developed for the improvement of homogeneous catalysis, and although they have been investigated in the hydration of nitriles and alkynes, they have not been applied to single-site catalytic alkene hydration.

The majority of the ligands employed in homogeneous catalysis are "innocent": they participate in neither the catalytic process itself nor in related redox reactions. ${ }^{60}$ However, spectator behaviour is not always desirable, particularly if the substrate and/or reactants are difficult to activate with transition metals. For example, relying on aqua ligands to react with coordinated substrates has a tendency to result in the 
formation of multinuclear complexes with bridging hydroxo ligands rather than the desired reaction. ${ }^{23}$

\subsubsection{Hydrogen Bonding}

Water is the perfect substrate with which to investigate catalysts bearing groups that are able to participate in hydrogen bonding. Activating water through hydrogen bonding or coordination to a Lewis acidic site may lower the energy barrier to oxidative addition.

Tyler et al. found that hydrogen bonding increased the rate of cyanohydrin and nitrile hydration. ${ }^{61}$ Ruthenium(II) arene complexes are common nitrile hydration catalysts but are unable to hydrate cyanohydrins; the presence of cyanide ions poisons the catalyst. ${ }^{61}$ Using tris(dimethylamino)phosphine ligands, catalyst poisoning did not occur. Instead, the addition of up to one equivalent of $\mathrm{KCN}$ resulted in an increase in the rate of hydration. It was thought that $\left[\mathrm{Ru}\left(\eta^{6}\right.\right.$-para-cymene $\left.)(\mathrm{CN})(\mathrm{Cl})\left(\mathrm{P}\left(\mathrm{NMe}_{2}\right)_{3}\right)\right]$ was forming, and instead of poisoning the catalyst, the electron-withdrawing cyanide ligands were facilitating attack of water on the nitrile substrate. ${ }^{61}$

The high rate of hydration could not be explained with steric and electronic arguments and instead was found to be due to hydrogen bonding effects. ${ }^{61}$ These aided the deprotonation of water, with the oxygen able to bind to the coordinated nitrile (Figure 1.7). ${ }^{61}$

A

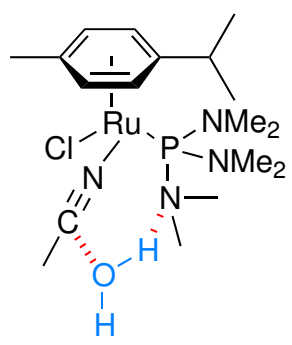

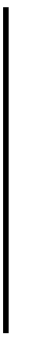
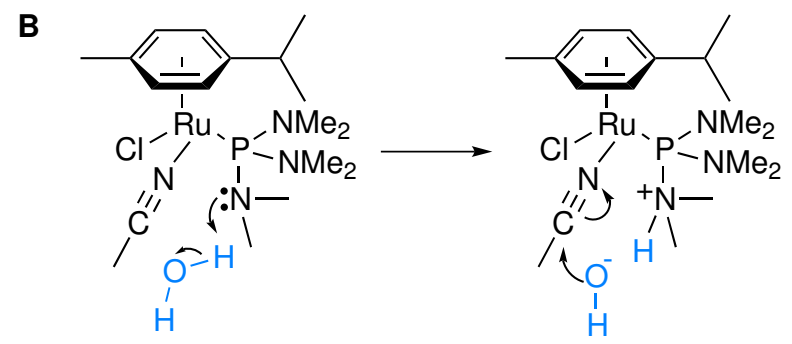

Figure 1.6 Concerted (A) and step-wise (B) nucleophilic attack of water on coordinated acetonitrile facilitated by a phosphine bearing a Lewis basic nitrogen atom. ${ }^{61}$

As mentioned in the discussion of nitrile hydration, rhodium is able to catalyse this reaction. The Bera group have reported a rhodium(I) NHC complex that is able to hydrate nitriles at room temperature by virtue of an uncoordinated naphthyridine group. ${ }^{26}$ The steps were modelled computationally and involve the transfer of a proton from water to the naphthyridine nitrogen and double hydrogen bonding interactions. 


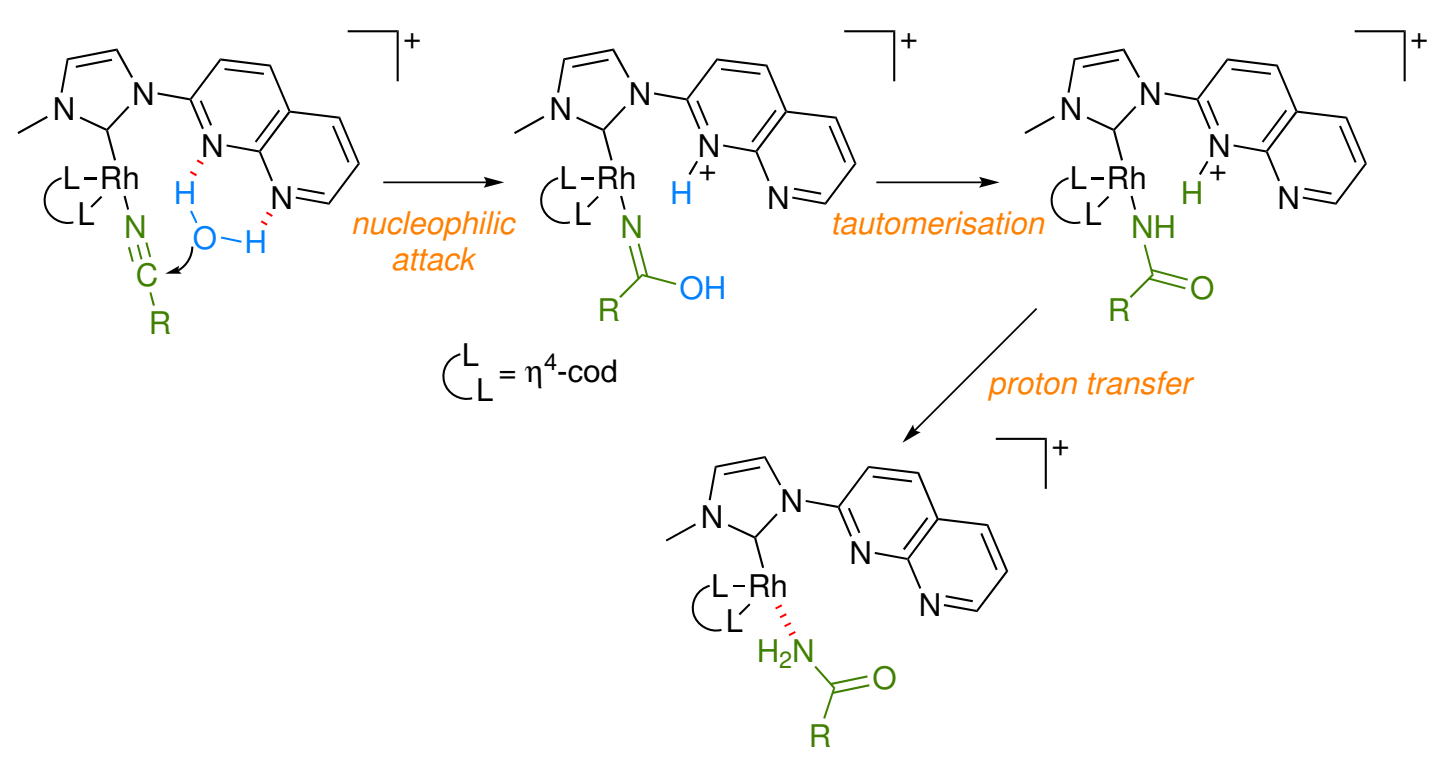

Figure 1.7 The first steps of nitrile hydration by a cooperative rhodium catalyst. $^{26}$

\subsubsection{Pyridylphosphines}

Pyridylphosphines are attractive ligands for cooperative catalysis due to the basicity of their nitrogen atoms and their ability to participate in hydrogen bonding interactions. Together, these features can also confer water solubility on pyridylphosphine complexes. ${ }^{62}$

Pyridylphosphines also have the potential to be very interesting from a coordination chemistry point of view - although usually coordinated through phosphorus, these $P, N$ ligands have the potential to bind to metals in a number of ways (Figure 1.8). ${ }^{63,64}$ The most well-studied of these ligands have 2-pyridyl substituents because their synthesis is more straightforward than that of the 3- and 4-pyridyl analogues. ${ }^{65}$ Interactions of pyridylphosphines with substrates are studied by NMR spectroscopy, IR spectroscopy, and computational chemistry.<smiles>[Y1]Pc1ccccn1</smiles><smiles></smiles><smiles></smiles><smiles></smiles>

Figure 1.8 Coordination modes of $\mathrm{Ph}_{2} \mathrm{P}(2$-pyr).

The ability of pyridylphosphines to adopt different coordination modes, particularly the $\kappa^{2}$ - $P, N$-chelate mode, is an important consideration when designing a catalyst. In an investigation into the hydration of benzonitrile by arene-ruthenium(II) complexes, complexes bearing pyridylphosphines displayed a strong tendency to adopt the chelating coordination mode (Figure 1.9). These complexes also showed low activity in the hydration of benzonitrile. ${ }^{66}$ 


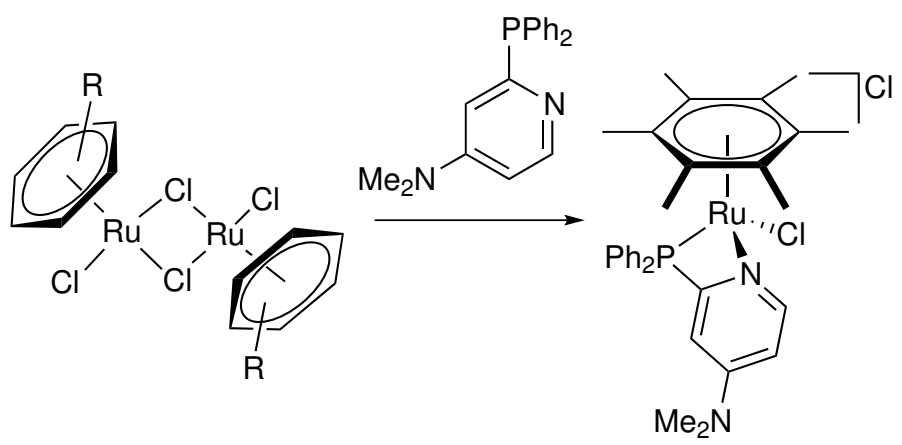

Figure 1.9 Direct formation of the $\kappa^{2}-P, N$ chelate. ${ }^{66}$

Grotjahn et al. developed a successful alkyne hydration system (it is unable to hydrate nitriles) by shielding the pyridyl nitrogen with a bulky tert-butyl group. This prevented $P, N$ chelation and resulted in an extremely active complex. ${ }^{67}$ Grotjahn's catalyst is noteworthy for its high selectivity and ability to generate up to a 10,000fold rate enhancement in the anti-Markovnikov hydration of alkynes. It has also been employed by the Herzon group in the tandem-catalytic synthesis of alcohols from terminal alkynes (Scheme 1.3, section 1.2). The substituted 2-pyridylphosphine ligands are able to form hydrogen bonds to terminal alkynes, activating them towards isomerisation (Figure 1.10). NMR spectroscopy provided good evidence for this interaction in the form of scalar coupling across the hydrogen bond between the substrate and the pyridyl ligand. This coupling disappeared in ruthenium complexes bearing triphenylphosphine ligands. ${ }^{68,69}$

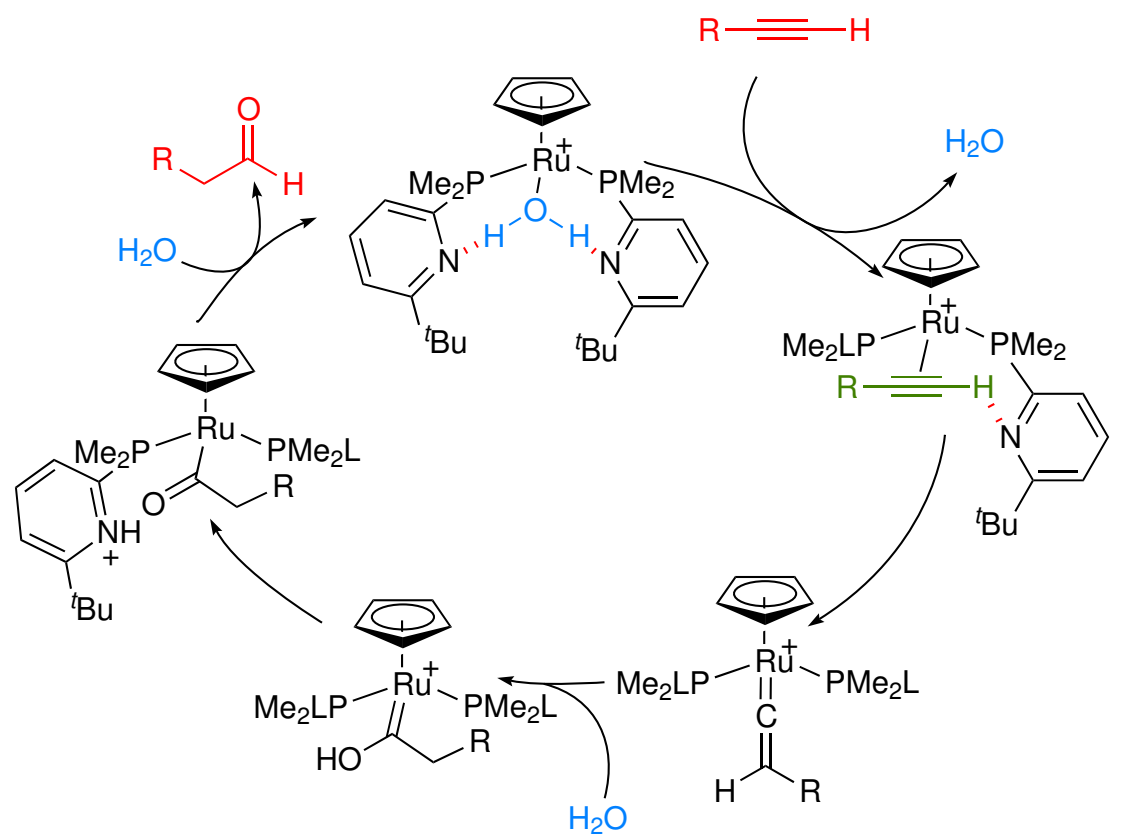

Figure 1.10 Anti-Markovnikov hydration of terminal alkynes catalysed by a ruthenium(II) complex bearing bulky 2-pyridylphosphines. $\mathrm{L}=6$-tert-butyl-2-diphenylphosphinylpyridine. ${ }^{68}$

This being said, pyridylphosphine complexes can still be highly active without modification of the pyridyl substituents. For example, Oshiki et al. reported that 
$\left[\right.$ cis- $\left.\mathrm{Ru}(\mathrm{acac})_{2}\left(\mathrm{PPh}_{2}(2-\mathrm{py})\right)\right]$ is able to catalyse the hydration of nitriles with a turnover frequency of up to $20,900 \mathrm{~h}^{-1} \cdot{ }^{70}$

\subsubsection{Aromatisation-Dearomatisation}

Small molecules can be activated by transition metal complexes without changes to the oxidation state of the metal. ${ }^{71}$ The Milstein group has developed pincer ligands with 2,6-substituted pyridine backbones able to carry out such reactions. The change in oxidation state occurs at nitrogen rather than at the metal. This feature may circumvent the problems associated with the oxidative addition of water such as the formation of stable aquo complexes. ${ }^{72}$ The classic example of cooperativity by such a ligand is the water-splitting catalyst developed by Milstein et al. ${ }^{51}$ As shown in Scheme 1.11, when a methylene spacer group is deprotonated, dearomatisation of the ligand backbone can occur. Rearomatisation occurs through reaction with the substrate (reversible reaction shown in blue).
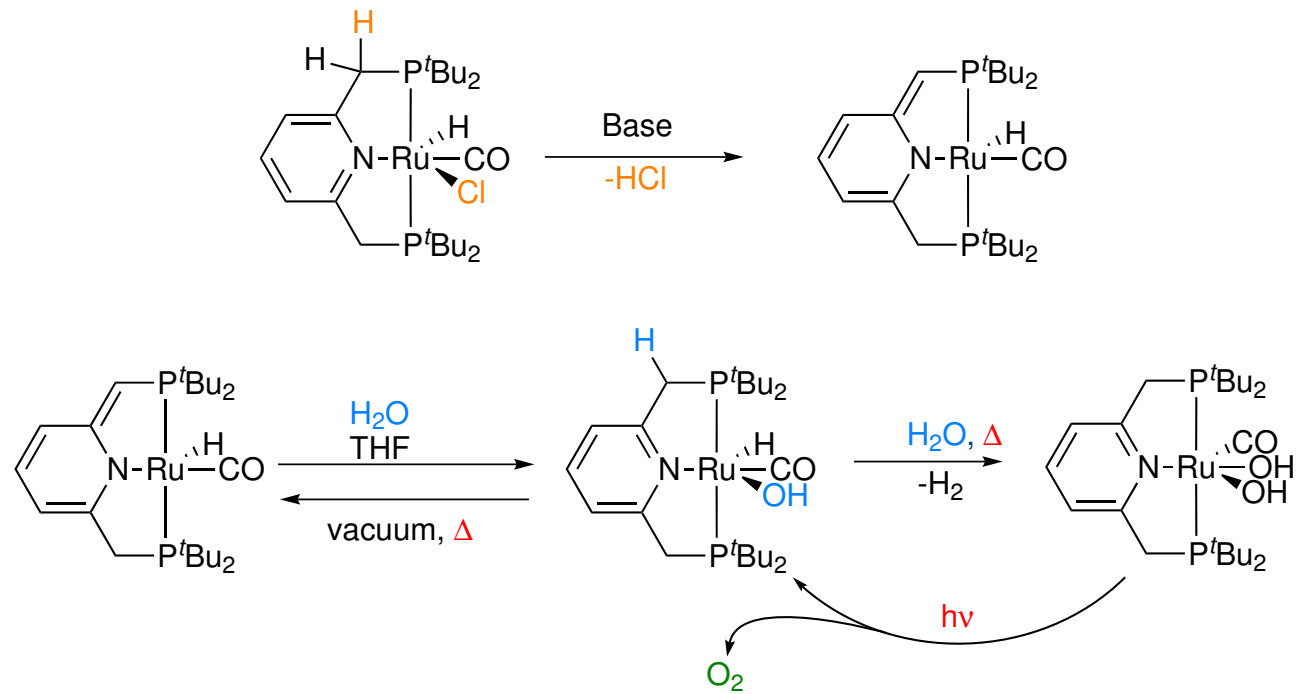

Scheme 1.11 Backbone dearomatisation was followed by a catalytic water splitting reaction promoted by both heat and light. ${ }^{51}$

\subsubsection{Lewis Acidic Sites}

Leitner and Bourissou et al. have recently shown that the combination of a metal hydride and a Lewis acidic site can activate dichloromethane, methanol, and dihydrogen. ${ }^{59}$ Incorporation of pendant borane groups into the well-defined coordination framework of the pincer ligand may open up new methods of activating difficult substrates such as water. These are synthesised by hydroboration of phosphines bearing unsaturated groups. Hydroboration of vinyl phosphines with 9-BBN has 
resulted in systems where traditional Lewis adducts do not form because of the steric bulk of the 9-BBN group. ${ }^{73}$ Figure 1.11 shows the general reactivity observed for the ruthenium complexes investigated by Leitner et al. ${ }^{74}$ These bifunctional hydride complexes hold the substrate close to the hydride, opening up the possibility for intramolecular transfer. ${ }^{59}$
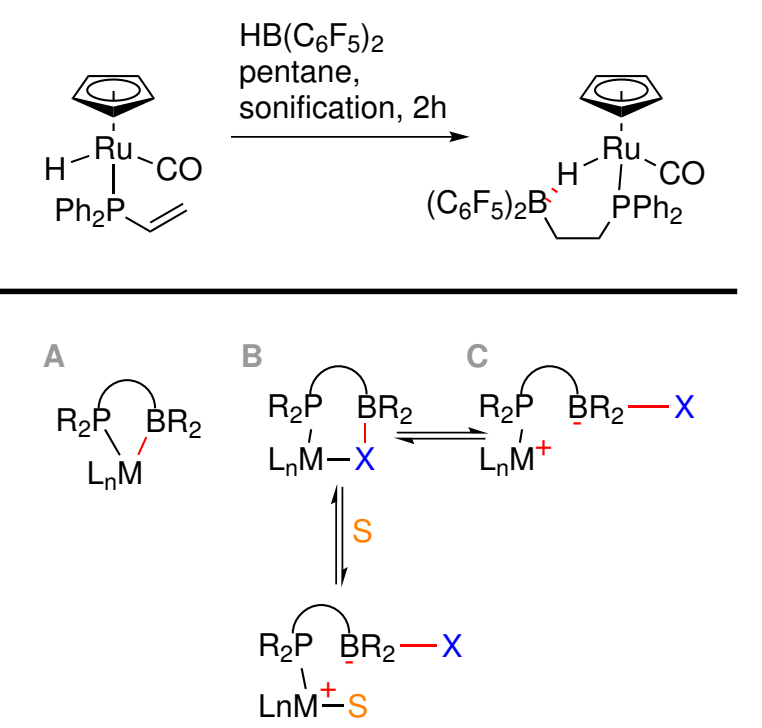

Figure 1.11 Top: Synthesis of a bifunctional ruthenium complex. ${ }^{59}$ Bottom: Pendant boranes have been observed to interact in a number of ways with metals and ligands. Leitner et al. observed interactions B and C, where $\mathrm{S}=$ methanol. ${ }^{59,74}$ 


\subsection{Research Objectives}

Research into the fundamental steps making up catalytic reactions has laid a foundation for the design of systems that can activate and use water. This reactivity is highly desirable as water is an environmentally friendly reagent. By employing ligands that can interact with substrates both bound to the metal and in the secondary coordination sphere, a number of successful hydration catalysts have been synthesised. Furthermore, certain complexes demonstrate water solubility, which is a particularly desirable feature for a hydration catalyst.

The pincer ligand framework is well-suited to catalytic applications due to its ability to form well-defined transition metal complexes. The ability of the PNP pincer ligand framework to undergo aromatisation/dearomatisation reactions has been exploited in the activation of strong bonds. However, the substituents on the phosphorus atoms of reported pincer ligands are all unable to interact with substrates.

The aims of this Masters research project are to synthesise novel PNP pincer ligands bearing substituents able to interact with water in a potentially "cooperative" fashion, such that hydration of unsaturated substrates can be investigated.

First, PNP ligands with 2-pyridyl and $-\mathrm{CH}_{2}-\mathrm{CH}_{2}-(9-\mathrm{BBN})$ groups will be synthesised along with the known ligands ${ }^{t} \mathrm{Bu} P N P$ and ${ }^{\mathrm{Ph}} \mathrm{PNP}$ for comparison. Next, the coordination chemistry of the novel ligands with iridium and rhodium will be investigated. These metals are able to take on a wide range of coordination geometries, have also shown promise in $\mathrm{O}-\mathrm{H}$ bond activation, and have been studied in pincer chemistry.

Finally, the ability of the resulting complexes to catalyse the hydration of alkenes, alkynes, and nitriles will be investigated. Particularly important will be to demonstrate whether the presence of pyridyl or borane groups results in increase an activity. The outcome of successful hydration will be an environmentally friendly method of transforming unsaturated substrates. 


\section{Chapter 2}

\section{Ligand Synthesis}

Pincer ligands are widely studied due to their well-defined coordination preferences, strong chelation, and the relative ease with which their steric and electronic properties can be modified. The highly modular synthesis of the pincer framework means that as well as being able to modify the backbone of the ligand, the substituents on the phosphorus atoms can be tailored to the desired application. For example, pyridine-derived backbones allow ligands such as ${ }^{\mathrm{R}} \mathrm{PCNCP},{ }^{\mathrm{R}} \mathrm{PONOP}$, and ${ }^{\mathrm{R}} \mathrm{PNNNP}$ to be synthesised. Substituents on such ligands span a wide range of electronic and steric properties, from electron-withdrawing $\mathrm{C}_{6} \mathrm{~F}_{5}$ groups through to bulky and electron-donating neopentyl groups. ${ }^{75,76}$

This work aimed to investigate the synthesis, properties, and catalytic activity of rhodium and iridium complexes bearing "cooperative" pincer ligands. The PCNCP framework* was selected due to the ability of the backbone to undergo dearomatisation when deprotonated at the methylene positions (see chapter one). Pendant borane and 2-pyridyl substituents were investigated for their ability to increase both the number and the type of interactions the ligand is able to have with water. Similar interactions have been reported to activate water molecules towards reaction with the metal centre and/or coordinated substrates.
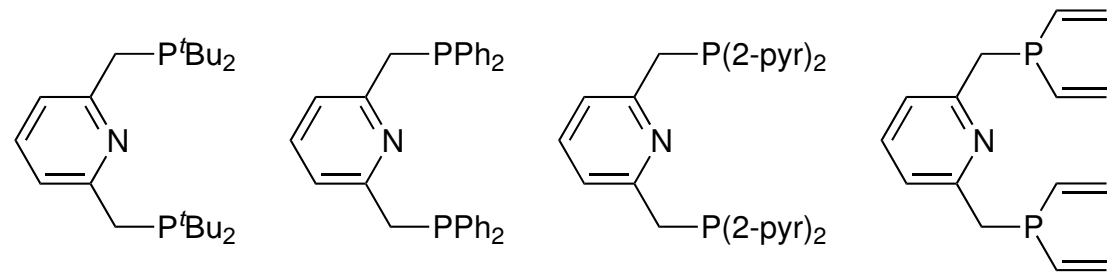

Figure 2.1 Target ligands ${ }^{t} \mathrm{Bu} \mathrm{PNP},{ }^{\mathrm{Ph}} \mathrm{PNP},{ }^{2-\mathrm{pyr}} \mathrm{PNP}$, and ${ }^{\mathrm{Vi}} \mathrm{PNP}$.

*Abbreviated as PNP in this thesis 
Pincer ligands of the class $R^{\prime}\left(\mathrm{PR}_{2}\right)_{2}$ (where $\mathrm{R}^{\prime}$ is the pincer ligand backbone) are synthesised by a wide variety of methods. These can be divided into two groups: those that form $\mathrm{P}-\mathrm{C}$ bonds between the backbone $\mathrm{R}^{\prime}$ and an " $\mathrm{PR}_{2}$ " group such as $\operatorname{LiP}(2 \text {-pyr })_{2}$, and those that install the desired substituents $\mathrm{R}$ in the end stages of the synthesis. This is achieved by modifying a $\mathrm{P}(\mathrm{III})$ or $\mathrm{P}(\mathrm{V})$-containing moiety bearing leaving groups such as $\mathrm{Cl}^{-}$or $\mathrm{OEt}^{-}$.

This chapter describes the synthesis of three diphosphine pincer ligands (Figure 2.1; ${ }^{t}$ Bu PNP, ${ }^{77}{ }^{\mathrm{Ph}} \mathrm{PNP},{ }^{78}$ and ${ }^{2-\mathrm{pyr}} \mathrm{PNP}, 2$-pyr $=2$-pyridyl), with the synthesis of ${ }^{2-p y r} \mathrm{PNP}$ having not yet been reported. Efforts towards the synthesis of the novel tetra-vinyl substituted PNP ligand ( ${ }^{V i} \mathrm{PNP}$ ) were met with some degree of success; however, hydroboration of ViPNP to install pendant Lewis-acidic groups posed an even greater challenge.

\subsection{Synthesis of ${ }^{t} \mathrm{Bu} \mathrm{PNP}$}

The first goal of this project was to synthesise 2,6-bis(di-tert-butylphosphinomethyl)pyridine $\left({ }^{t} \mathrm{Bu} P N P, 6\right)$. Instead of purchasing this ligand from a commercial supplier, a new synthetic method was investigated. The three published synthetic routes to 6 are summarised in Scheme 2.1. ${ }^{77,79,80}$ This illustrates the variety of methods available for synthesising phosphine ligands. 


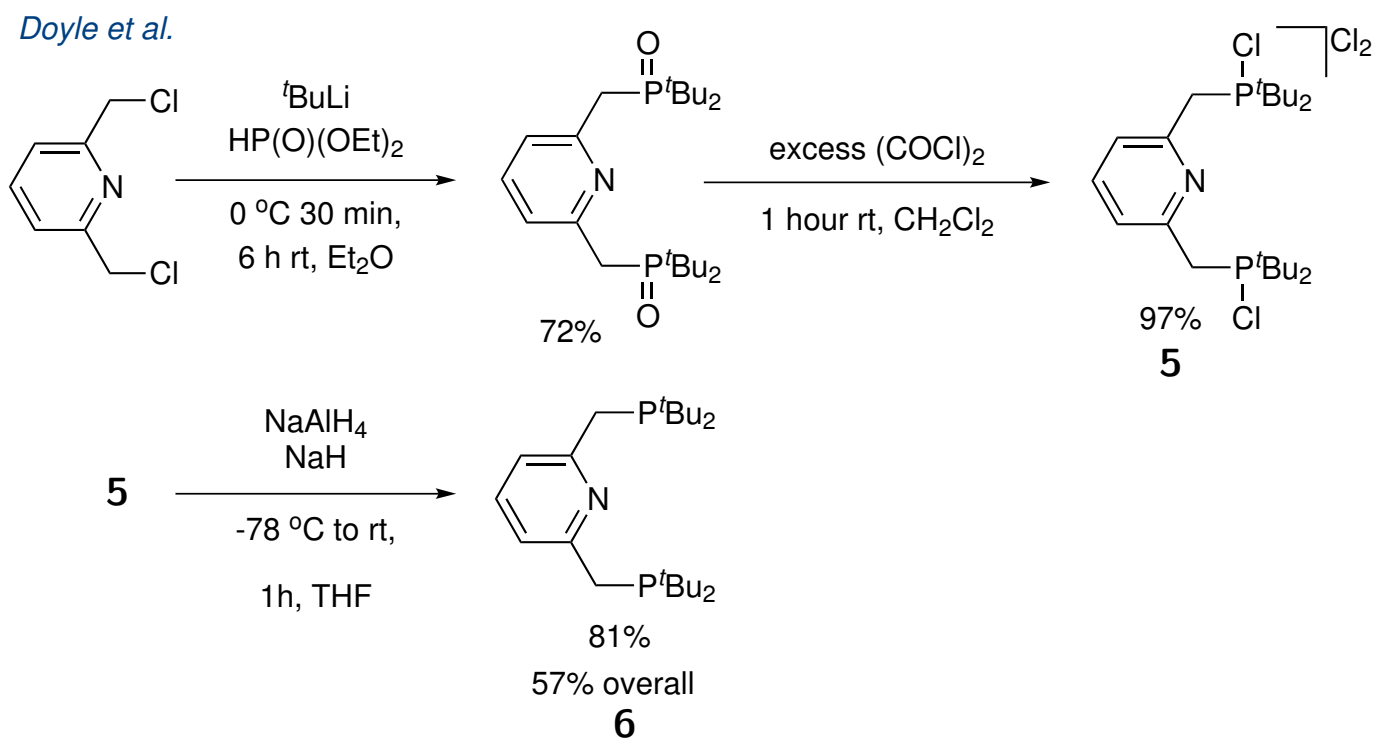

Hartwig

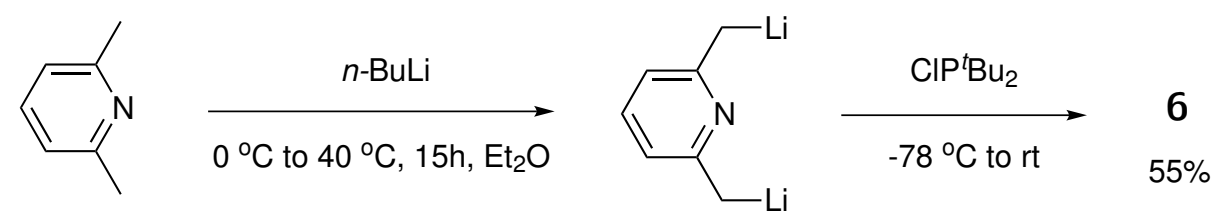

Milstein et al.
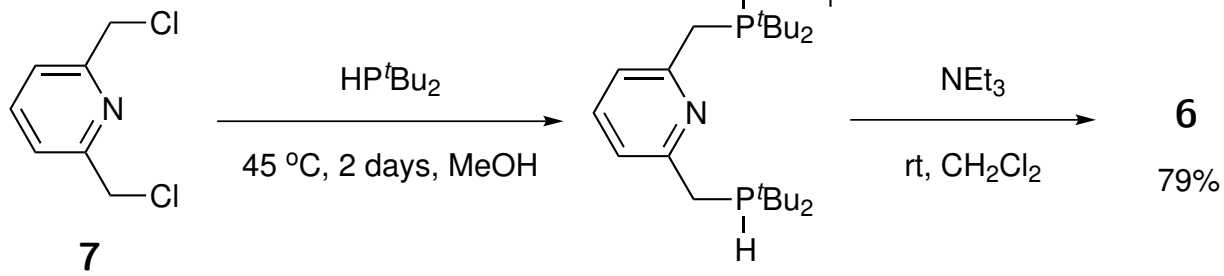

Scheme 2.1 Existing methods for the synthesis of $\mathbf{6}^{77,79,80}$ 
An alternative route to ${ }^{t}$ Bu PNP via the bis(borane) adduct 8 was investigated (Scheme 2.2). This compound has not been reported, but as phosphine-boranes are air- and moisture-stable, synthesising 8 would be a convenient way of storing 6 until it is required.

The air-stable phosphine-borane ${ }^{t} \mathrm{Bu}_{2} \mathrm{PH}\left(\mathrm{BH}_{3}\right)$ was synthesised in good yield by reacting ${ }^{t} \mathrm{Bu}_{2} \mathrm{PCl}$ with excess $\mathrm{NaBH}_{4}$. Reduction and protection of the phosphine with $\mathrm{NaBH}_{4}$ is a one-step reaction and avoids the low-yielding synthesis of ${ }^{t} \mathrm{Bu}_{2} \mathrm{PH}$ required to carry out the Milstein synthesis (Scheme 2.1). ${ }^{79}$ The electrophilic 2,6substituted pyridine backbone was synthesised from pyridine dihyroxymethanol by bromination using $\mathrm{PBr}_{3}$ or chlorination using thionyl chloride. ${ }^{81,82}$ Reaction of ${ }^{t} \mathrm{Bu}_{2} \mathrm{PLi}\left(\mathrm{BH}_{3}\right)$ with 2,6-bis(chloromethyl)pyridine (7) gave an air-stable solid that was purified by recrystallisation from hexane (Scheme 2.2).

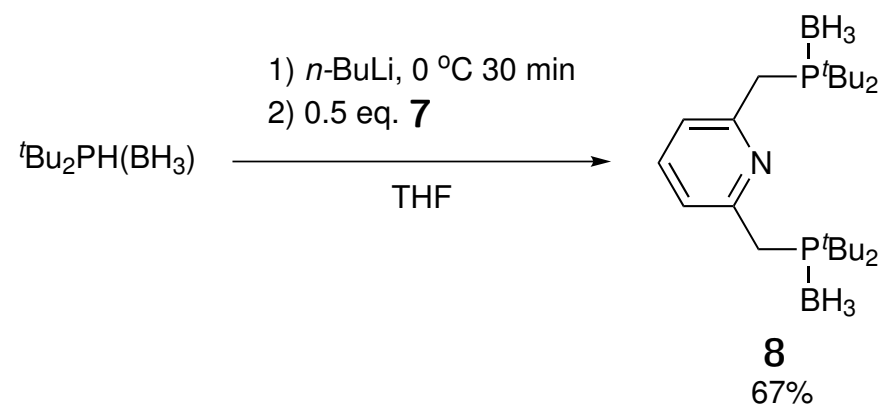

Scheme 2.2 Synthesis of borane-protected ${ }^{t}$ Bu PNP, 8.

The use of 2,6-bis(bromomethyl)pyridine (9) instead of the chloro analogue $\mathbf{7}$ resulted in a mixture of products and a low yield of 8 . The ${ }^{31} \mathrm{P}\left\{{ }^{1} \mathrm{H}\right\}$ NMR spectrum displayed many signals, including a broadened multiplet at $\delta_{P} 148 \mathrm{ppm}$. This chemical shift suggested that ${ }^{t} \mathrm{Bu}_{2} \mathrm{PBr}\left(\mathrm{BH}_{3}\right)$ had formed. A signal at $\delta_{P} 148 \mathrm{ppm}$ is approximately 100 ppm downfield from where it would be expected had only 8 formed, and is evidence for the presence of a bond between phosphorus and an electron-withdrawing group. The shape of the signal suggested the presence of a $\mathrm{P}_{-} \mathrm{BH}_{3}$ bond; when ${ }^{31} \mathrm{P}$ is coupled to the quadrupolar NMR-active isotopes of boron $\left({ }^{10} \mathrm{~B}\right.$ and $\left.{ }^{11} \mathrm{~B}\right)$ a broad multiplet will be observed.

Lithium-bromine exchange has been shown to affect lithiation reactions. ${ }^{83,84}$ In fact, Imamoto et al. exploited this to synthesise $(S)$-(tert-butylmethylbromophosphine)borane (10) from $(S)$-(tert-butylmethylphosphanyl)borane* as a precursor to P-chiral alkynyl phosphines (Scheme 2.3). ${ }^{85}$ Compound 10 is the only reported phosphineborane containing a $\mathrm{P}-\mathrm{Br}$ bond. To confirm lithium-bromine exchange was responsible

${ }^{*}$ The substituent name -phosphanyl has been used here as it is recommended by IUPAC for naming groups derived from $\mathrm{PH}_{3}$. This contrasts with the use of "phosphine" rather than "phosphane" throughout this thesis; however, there is the potential for ambiguity here as "phosphinyl" can also mean $\mathrm{H}_{2} \mathrm{P}(\mathrm{O})$-. 
for the low yield of $\mathbf{8},{ }^{t} \mathrm{Bu}_{2} \mathrm{PLi}\left(\mathrm{BH}_{3}\right)$ was reacted with 1,2-dibromoethane. This acted as the bromine source, rather than 9. The resulting ${ }^{31} \mathrm{P}\left\{{ }^{1} \mathrm{H}\right\}$ NMR spectrum contained a signal at the same chemical shift as the peak that resulted from the unsuccessful ligand synthesis reaction.

This work:

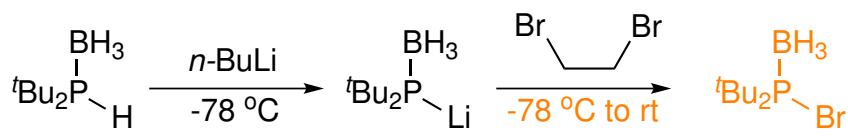

Imamoto et al.:

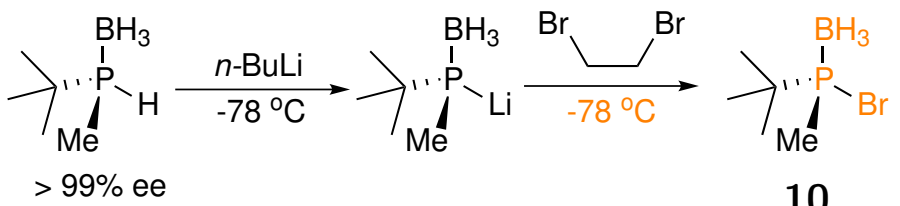

Scheme 2.3 Lithium-bromine exchange.

In order to coordinate 8 to late transition metals the borane groups must be removed. Phosphine-boranes are deprotected under a number of different conditions. ${ }^{86,87}$ Heating in neat morpholine has been found to be successful for a wide variety of ligands, so this method was investigated first. ${ }^{88}$ The reaction outlined in Scheme 2.4 was carried out and gave ${ }^{t}$ Bu PNP (6) in $86 \%$ yield. NMR spectra matched those reported by Milstein et al. ${ }^{79}$

As only a small amount of 6 was required for comparison with ${ }^{2-p y r} \mathrm{PNP}$, the two-step synthesis outlined in Scheme 2.1 and Scheme 2.4 was only required once. This meant that the reaction conditions were not optimised. However, ${ }^{t} \mathrm{Bu} P N P$ was still obtained in a comparable yield to the Hartwig and Doyle routes (58\%). This new route also took less than two days, did not require heating, and did not require column chromatography.

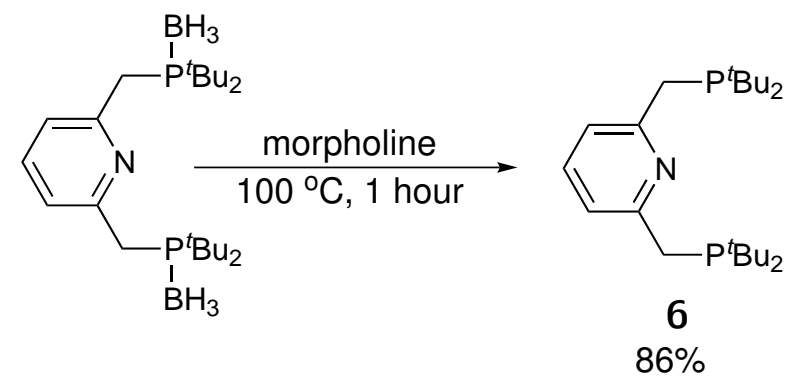

Scheme 2.4 Deprotection of borane-protected ${ }^{t}$ BuPNP. 


\subsection{Synthesis of a Vinyl-Substituted PNP Ligand}

Pendant borane groups have yet to be incorporated into PNP pincer ligands, and the stability of such molecules has yet to be determined. Boranes are incorporated into other classes of ligand by hydroboration of phosphines bearing alkenyl substituents. ${ }^{59,89,90}$ Therefore, the direct precursor to a ligand bearing pendant borane groups with $-\mathrm{CH}_{2} \mathrm{CH}_{2}-$ linkers would be the tetra-vinyl-substituted PNP ligand ${ }^{\mathrm{Vi}} \mathrm{PNP}(\mathbf{1 1})$.

A number of synthetic routes to alkyl and aryl phosphines are known, but the literature describing the synthesis of alkenyl phosphines is much more limited. ${ }^{91}$ Attachment of the bis(vinyl)phosphine fragment to the pyridine backbone, in a similar fashion to the Hartwig or Milstein syntheses of ${ }^{t}$ Bu PNP, would have been the simplest method of making ViPNP (Scheme 2.5). ${ }^{77,79}$ However, the syntheses of divinylphosphine and divinylphosphine chloride are neither well-established nor particularly straightforward. ${ }^{92-94}$ Both phosphines are low-boiling liquids and are likely to be highly reactive. ${ }^{93}$ In fact, even trivinyl phosphine can only be synthesised in moderate yields, as many side reactions occur. ${ }^{95}$
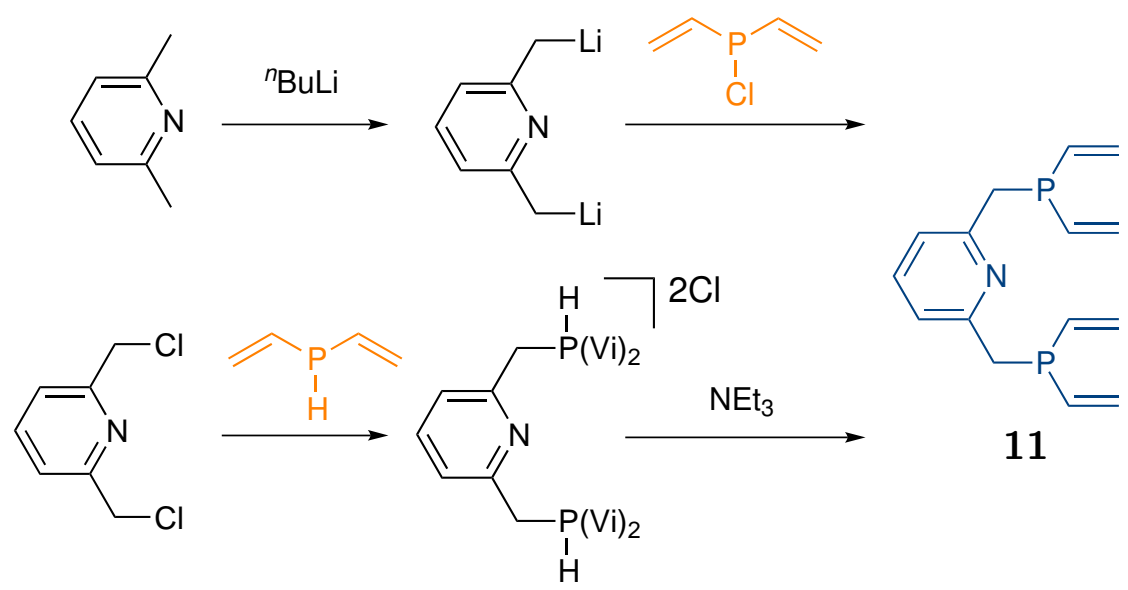

Scheme 2.5 Possible routes to ${ }^{\mathrm{V}} \mathrm{PNP}$ based on literature syntheses of ${ }^{t}$ BupNP. ${ }^{77,79}$

As a consequence, efforts towards the synthesis of ViPNP concentrated on forming the $\mathrm{P}$-backbone bond before the $\mathrm{P}$-vinyl bonds. The success of this route rested on the installation of suitable leaving groups on phosphorus so that substitution with vinylmagnesium bromide could occur.

The Michaelis-Arbuzov reaction is a high-yielding method for the synthesis of $\mathrm{P}-\mathrm{C}$ bonds. ${ }^{96}$ It requires very simple starting materials - an alkyl halide and a trialkyl phosphite - and forms a phosphonate. This phosphorus(V) species can then be reacted further to form phosphine ligands. For example, Roddick et al. successfully 
synthesised trifluoromethyl-substituted PCP ligand 12 via the phosphonate intermediate 13 (Scheme 2.6). A Michaelis-Arbuzov reaction was used to form the $\mathrm{P}-\mathrm{C}$ bond in 13, and reduction of this phosphorus $(\mathrm{V})$ species followed by chlorination (14) and substitution resulted in the desired product $12 .{ }^{97}$
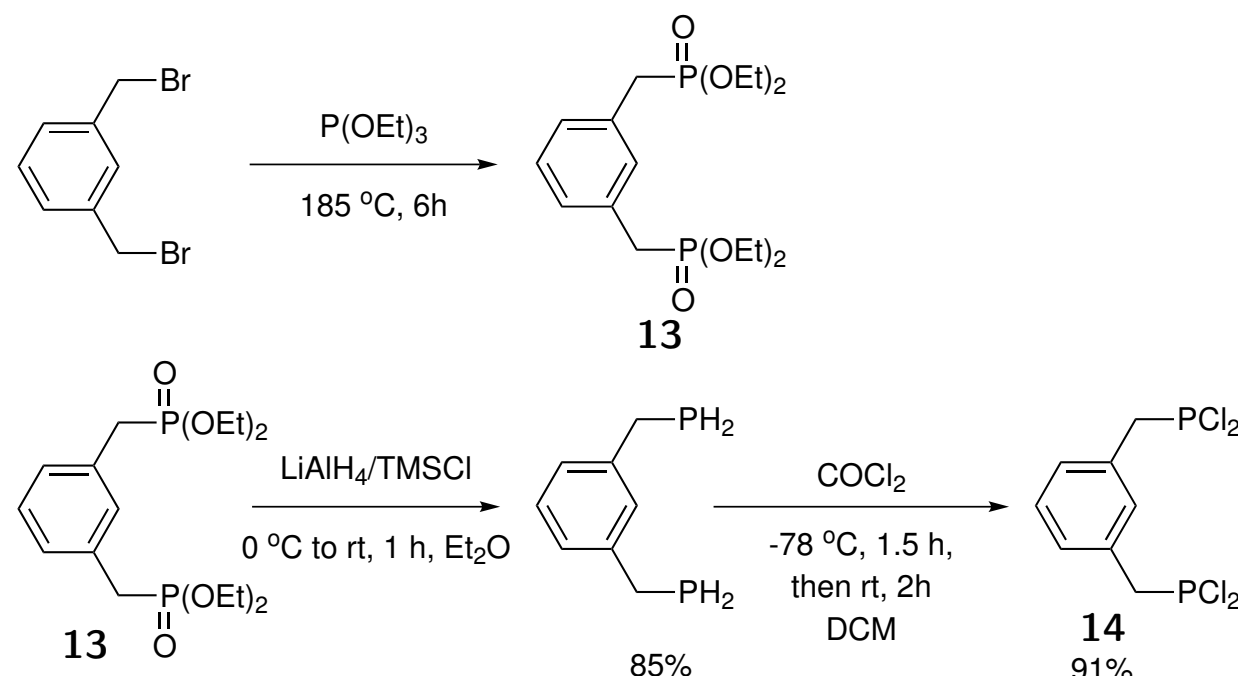

14

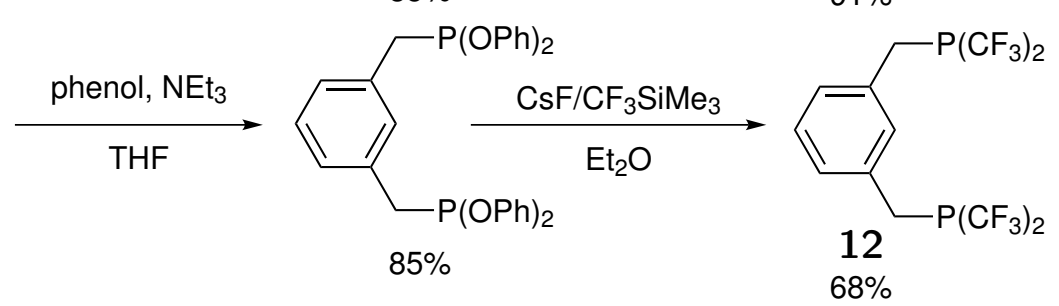

Scheme 2.6 Synthesis of $\mathrm{CF}_{3} \mathrm{PCP}$ by Roddick et al.. ${ }^{97}$

The first two steps in the synthesis of $\mathrm{CF}_{3} \mathrm{PCP}(\mathbf{1 2})$ were repeated with the PNP analogue 2,6-bis[(diethoxyphosphoryl)methyl]pyridine (15), which was synthesised in quantitive yield by heating 2,6-bis(chloromethyl)pyridine $\mathbf{7}$ in triethyl phosphite at $140{ }^{\circ} \mathrm{C}$ for 3 hours. ${ }^{98,99}$ However, the chlorination conditions reported by Roddick et al. were modified so that $\mathrm{PCl}_{5}$ could be used instead of phosgene. ${ }^{100}$ Neither phosgene nor its safer precursor triphosgene were available for this work.

Reaction of $\mathbf{1 5}$ with a strongly reducing mixture of $\mathrm{TMSCl} / \mathrm{LiAlH}_{4}$ resulted in pure 2,6-bis(phosphinomethyl)pyridine ${ }^{\mathrm{H}} \mathrm{PNP}$ (16, Figure 2.2). Neither 16 nor ${ }^{\mathrm{Cl}} \mathrm{PNP}$ have been reported. The NMR data of $\mathbf{1 6}$ were similar to those reported by Roddick et al.; the ${ }^{31} \mathrm{P}\left\{{ }^{1} \mathrm{H}\right\}$ NMR spectrum had a single resonance at $\delta_{P}-131 \mathrm{ppm}$, and the hydrogen atoms attached to phosphorus displayed $\mathrm{P}-\mathrm{H}$ coupling (doublet of triplets, $\left.{ }^{1} J_{\mathrm{P}-\mathrm{H}}=192 \mathrm{~Hz},{ }^{2} J_{\mathrm{H}-\mathrm{H}}=7.7 \mathrm{~Hz}\right)$.

The primary phosphine ${ }^{\mathrm{H}} \mathrm{PNP}$ is extremely unstable, reacting in diethyl ether solution to form a solid that is insoluble in $\mathrm{C}_{6} \mathrm{D}_{6}, \mathrm{CDCl}_{3}$, acetone, and $\mathrm{H}_{2} \mathrm{O}$. In order to have enough material with which to investigate the chlorination reaction, ${ }^{\mathrm{H}} \mathrm{PNP}$ was not isolated in subsequent syntheses. Instead, the freshly prepared ethereal solution of $\mathbf{1 6}$ was transferred by cannula into a suspension of $\mathrm{PCl}_{5}$ in diethyl ether. It was hoped 
that the transfer would take place fast enough that the majority of $\mathbf{1 6}$ would remain intact. Unfortunately, although the material formed in this reaction was analysed by NMR spectroscopy in both $\mathrm{CDCl}_{3}$ and $\mathrm{C}_{6} \mathrm{D}_{6}$, no ${ }^{31} \mathrm{P}\left\{{ }^{1} \mathrm{H}\right\}$ NMR signals or pyridyl protons could be found. This is could have been due to a number of factors, including the decomposition of $\mathbf{1 6}$, and $\mathrm{P}-\mathrm{C}$ oxidative cleavage (where a $\mathrm{P}-\mathrm{C}$ bond becomes a $\mathrm{Cl}-\mathrm{C}$ bond), which has been reported under similar chlorination conditions. ${ }^{100}$

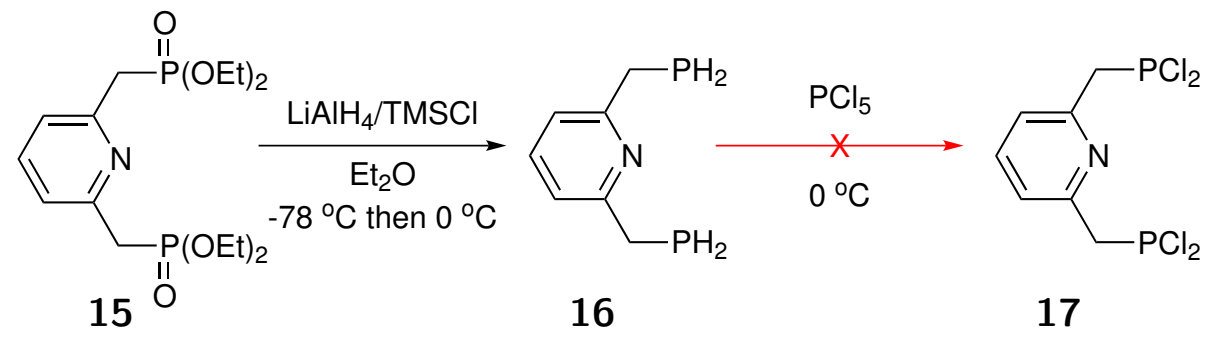

Figure 2.2 Attempted synthesis of ${ }^{\mathrm{Cl}} \mathrm{PNP}(\mathbf{1 7})$.

Because $\mathbf{1 7}$ required the synthesis of highly unstable intermediate 16, an alternative route to ${ }^{\mathrm{Vi}} \mathrm{PNP}$ involving an ethoxy-substituted phosphonite $\left(\mathrm{RP}(\mathrm{OEt})_{2}\right)$ became the next target. Phosphonite synthesis can be achieved through reaction of a Grignard or organolithium reagent with diethyl chlorophosphite or triethyl phosphite. The yields of such reactions are generally high. ${ }^{89,95}$

Reactions of Grignard reagents with $\mathrm{P}-\mathrm{O}$ bonds are very common, and such reactions are also involved in the synthesis of vinyl-substituted phosphines. ${ }^{89,95}$ For example, Scheme 2.7 summarises the synthesis of divinylphenylphosphine reported by GilbertWilson et al. ${ }^{89}$ To prevent polymerisation, the product was not isolated from the ether solution. 1,4-Dioxane was added to precipitate the magnesium salts and excess vinylmagnesium bromide. ${ }^{89}$<smiles>C=CP(C=C)c1ccccc1</smiles>

Scheme 2.7 Synthesis of a tertiary phosphine from a phosphonite. ${ }^{89}$

As shown in Scheme 2.8, reaction of diethyl chlorophosphite with di-Grignard reagent 18 (derived from 2,6-bis(bromomethyl)pyridine) should furnish ${ }^{\text {OEt} P N P} 19$ (Scheme 2.8). This compound has never been reported, and the synthesis of $\mathbf{1 8}$ has only been reported twice. Both times magnesium-anthracene (20) was employed as a soluble source of magnesium. ${ }^{101}$ However, the synthesis of $\mathbf{2 0}$ is not reliable and the removal of anthracene from the product can sometimes be difficult. Thus, the alternative bis(nucleophile) $\mathbf{2 1}$ was generated from 2,6-dimethylpyridine using $n$-BuLi. Compound $\mathbf{2 1}$ was reacted with triethyl phosphite, followed by vinylmagnesium bromide. The ${ }^{31} \mathrm{P}\left\{{ }^{1} \mathrm{H}\right\}$ NMR spectrum of the resulting dark red THF/diethyl ether 
solution contained two signals in a 1:1 ratio at $\delta_{P}-18.2$ and $-18.6 \mathrm{ppm}$. It is possible that these result from incomplete substitution of the P-OEt groups for vinyl groups. The ${ }^{1} \mathrm{H}$ NMR spectrum of the crude reaction mixture showed the presence of excess vinylmagnesium bromide and well-resolved multiplets at 5.62, 6.18, and $6.24 \mathrm{ppm}$. These resonances resulted from the presence of $\mathrm{P}-\mathrm{Vi}$ groups. However, there were also two overlapping sets of resonances for the pyridine backbone of the ligand, which was consistent with the presence of two signals in the ${ }^{31} \mathrm{P}\left\{{ }^{1} \mathrm{H}\right\}$ NMR spectrum. This was confirmed by synthesising the known compound $\mathrm{Vi}_{2} \mathrm{P}\left(\mathrm{NEt}_{2}\right)$ and comparing the ${ }^{1} \mathrm{H}$ NMR spectra. Following the methodology developed by Gilbert-Wilson et al., magnesium salts were precipitated by the addition of 1,4-dioxane, and volatiles were not removed to prevent polymerisation. ${ }^{89}$

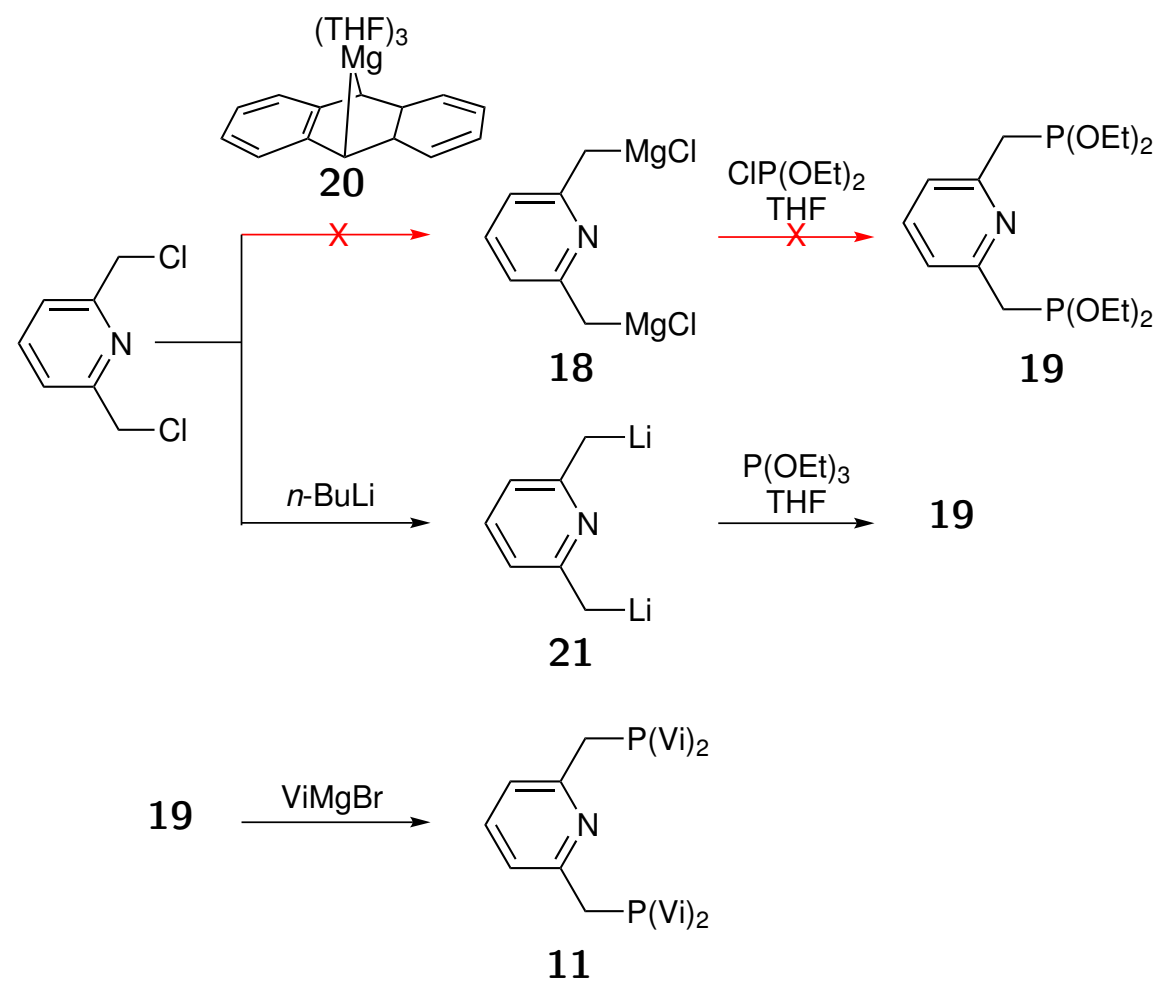

Scheme 2.8 Synthesis of pincer ligand ${ }^{\mathrm{V}} \mathrm{PNP}$.

\subsubsection{Hydroboration of ${ }^{\mathrm{Vi}} \mathrm{PNP}$}

Hydroboration of the vinyl groups was investigated (Scheme 2.9). 9-Borabicyclo[3.3.1]nonane (9-BBN) was added to the crude ethereal solution of ViPNP described above. The solution was heated at $45^{\circ} \mathrm{C}$ for 5 hours then analysed by multinuclear NMR spectroscopy. This reaction was based on a method reported by Bourissou et al. ${ }^{73}$

${ }^{1} \mathrm{H}$ NMR spectra obtained from aliquots of the solution $\left(2: 1 \mathrm{THF} / \mathrm{THF}-\mathrm{d}_{8}\right)$ showed the absence of vinylic resonances. The ${ }^{31} \mathrm{P}\left\{{ }^{1} \mathrm{H}\right\}$ NMR spectrum contained two signals: a singlet at $32.7 \mathrm{ppm}$ and a broad peak centred on $1 \mathrm{ppm}$. The ${ }^{11} \mathrm{~B}$ NMR 
<smiles>C=CP(C=C)Cc1cccc(CP(C=C)C=C)n1</smiles>
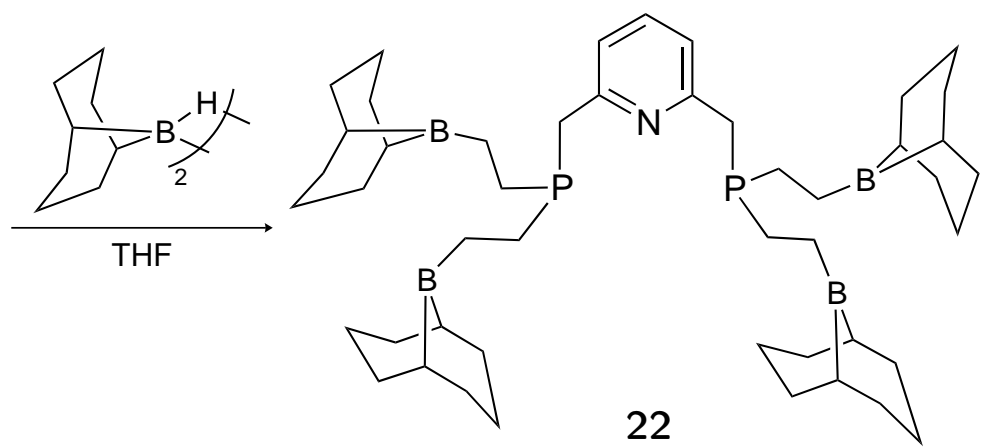

Scheme 2.9 Attempted synthesis of pendant Lewis acid-containing ligand 22 via hydroboration.

spectrum contained a number of signals. Unfortunately, although the THF could be evaporated and the residue re-dissolved in THF, the solution gelled after a night at room temperature. This prevented further characterisation and purification.

Phosphines bearing unsaturated alkyl groups have successfully undergone hydroboration both as the free ligand and whilst coordinated to a metal. ${ }^{59,90,102}$ However, none of these systems required the installation of four Lewis acidic groups onto the same molecule, nor did they contain a nitrogen atom. Thus, the countless intra- and inter-molecular interactions between phosphorus, nitrogen, boron, and THF in this particular system make 22 much more difficult to work with. Because of this, efforts were focussed on the synthesis and coordination chemistry of the PNP pincer ligand bearing 2-pyridyl groups $\left({ }^{2-p y r} \mathrm{PNP}\right)$.

\subsection{Synthesis of ${ }^{2-p y r} \mathrm{PNP}$}

\subsubsection{Overview}

Common routes to 2-pyridylphosphines require either the reaction of 2-pyridyllithium with chlorophosphines, or employ tris(2-pyridyl)phosphine as the source of $\mathrm{P}$-pyridyl groups. ${ }^{65}$ At the beginning of this project, the latter method was being used by Vaughan to synthesise 2-pyridyl-substituted diphosphine ligands (Scheme 2.10). ${ }^{103}$ However, neither the ligands in Scheme 2.10 nor ${ }^{2-p y r}$ PNP could be be obtained in particularly high purity. The lack of purity is clear in the ${ }^{1} \mathrm{H}$ NMR spectrum of crude ${ }^{2-p y r} \mathrm{PNP}$ shown in Figure 2.3.

Scheme 2.10 shows the transformations involved in the unoptimised synthetic route. First, a 2-pyridyl group is cleaved from $\mathrm{P}(2 \text {-pyr })_{3}$ using potassium. Next, the pyridyl 
anion byproduct is quenched with tert-butyl chloride. This results in a solution of nucleophilic $\mathrm{KP}(2 \text {-pyr })_{2}$, which can be reacted with an alkyl halide to form a new $\mathrm{P}-\mathrm{C}$ bond.<smiles>c1ccc(P(c2ccccn2)c2ccccn2)nc1</smiles>
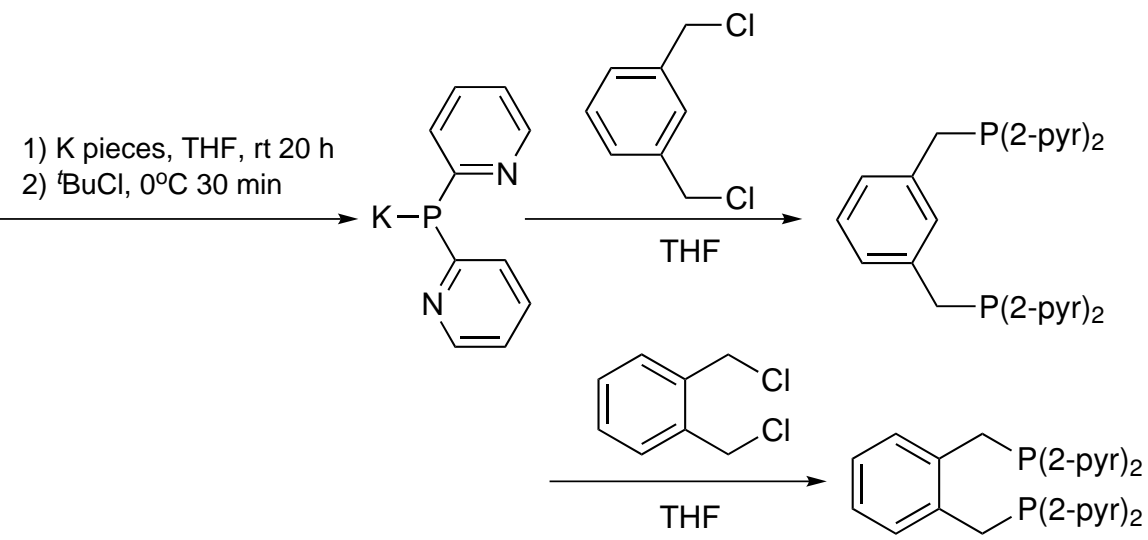

Scheme 2.10 The original synthetic route and the ligands it has been used to synthesise. ${ }^{103}$

Even after repeating the synthesis outlined in Scheme 2.10 a number of times, impure ${ }^{2-\text { pyr }} \mathrm{PNP}$ was still obtained. A ${ }^{1} \mathrm{H}$ NMR spectrum of such a sample is shown in Figure 2.3. Purification of the ${ }^{2-p y r} \mathrm{PNP}$ ligand synthesised by this route was unsuccessful.

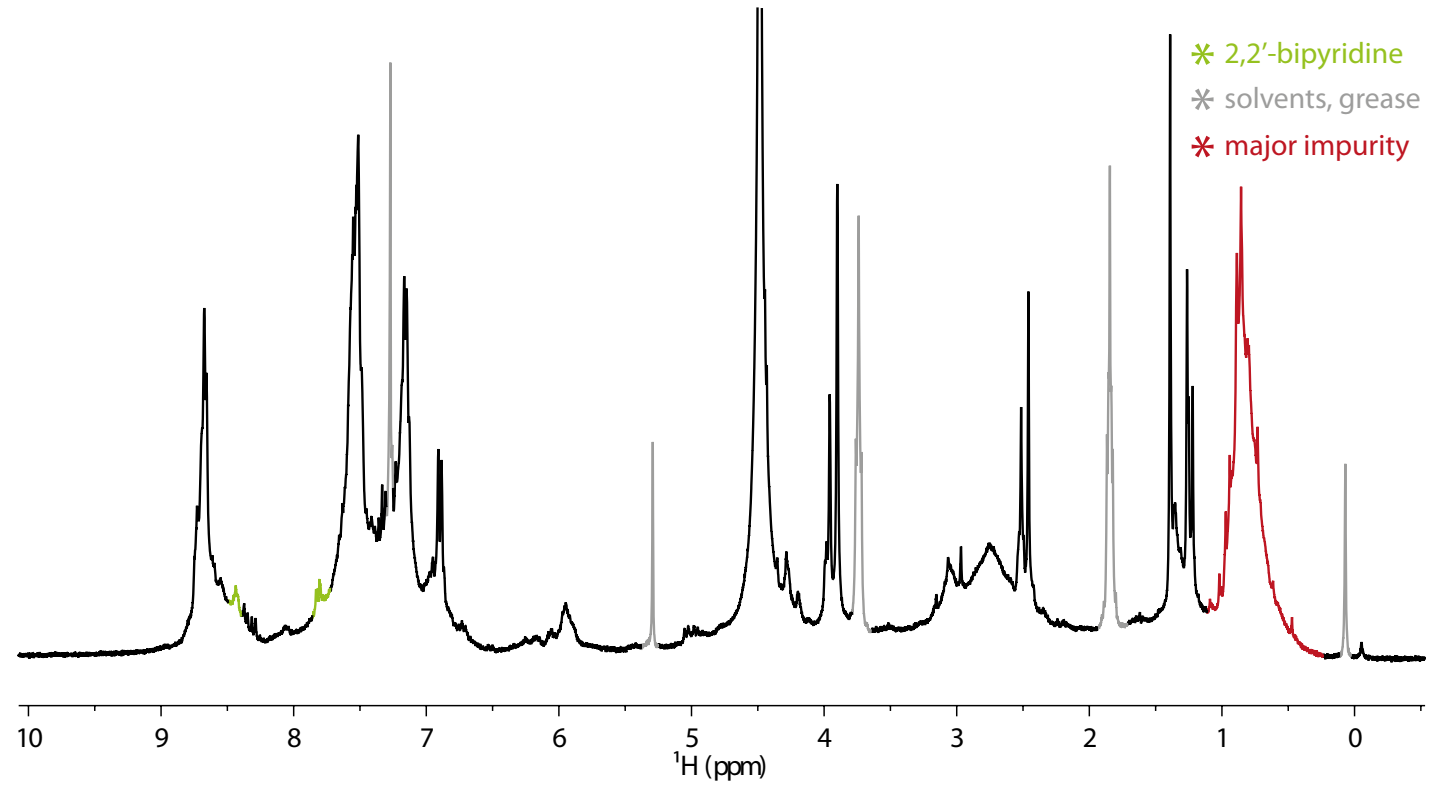

Figure 2.3 ${ }^{1} \mathrm{H}$ NMR spectrum of the first attempt at synthesising ${ }^{2-p y r} \mathrm{PNP}$.

The ${ }^{2-p y r}$ PNP ligand is basic and can be extracted into water by protonation with $\mathrm{HCl}$. Unfortunately, the vast majority of the impurities are also basic and this did not remove them. Washing with N-methylformamide to remove any highly polar impurities was also unsuccessful. Furthermore, the yield of ${ }^{2-p y r} \mathrm{PNP}$ dramatically decreased when it was passed through alumina or silica. Recrystallisations from hexane/n-propanol, $n$-pentane/pyridine, and ethanol/n-propanol were attempted 
but did not yield pure ${ }^{2-p y r} \mathrm{PNP}$.

As the ${ }^{2-p y r}$ PNP synthesised in this way resisted purification, it was essential that either a better synthetic method be found, or the current method optimised, if this project was to be successful. Initially, the viability of the latter option was investigated.

\subsubsection{Step 1: Cleavage of a $\mathrm{P}-(2$-pyridyl $)$ Bond}

Cleavage of $\mathrm{P}$-aryl groups using potassium, sodium, or lithium (M) is a wellestablished route to nucleophilic phosphide anions, and all three metals are able to cleave a pyridyl group from $\mathrm{P}(2 \text {-pyr })_{3}$ in THF to form $\mathrm{MP}(2-\mathrm{pyr})_{2} \cdot{ }^{103}$ However, organo-alkali byproducts such as $\mathrm{M}(2$-pyridyl) and $\mathrm{M}$ (phenyl) can also react with THF, and this reactivity increases from lithium to potassium. ${ }^{104}$ As 20 hours were required for the cleavage of the $\mathrm{P}$-(2-pyridyl) bond using potassium pieces, the reaction of THF with $\mathrm{K}$ (2-pyridyl) was hypothesised to be in part responsible for the presence of impurities in ${ }^{2-p y r} \mathrm{PNP}$.

The impurity highlighted in red in Figure 2.3 could be extracted into aqueous solution by addition of dilute $\mathrm{HCl}$. This suggested that basic 2-pyridyl anions were involved in its formation. Consequently, if the reaction time could be decreased and the alkali metal changed to lithium (which forms less reactive organometallic species), reactions of 2-pyridyl anions with the solvent would be less likely.

Investigations by Vaughan showed that high sodium lithium granules required six hours of sonication to react completely with $\mathrm{P}(2 \text {-pyr })_{3} \cdot{ }^{103}$ In this work, three equivalents of high sodium lithium powder were used and the reaction was complete within 1 hour. This was confirmed by reacting a small sample of $\operatorname{LiP}(2-\mathrm{pyr})_{2}$ (and any unreacted $\left.\mathrm{P}(2 \text {-pyr })_{3}\right)$ with water, and extracting the organic residue into $\mathrm{CDCl}_{3}$. The important features of the resulting NMR spectra are a singlet at $\delta_{P}-34 \mathrm{ppm}$, and a doublet centred on $\delta_{H} 5.53 \mathrm{ppm}\left({ }^{1} J_{\mathrm{P}-\mathrm{H}}=226 \mathrm{~Hz}\right)$ due to the $\mathrm{P}-\mathrm{H}$ bond of the secondary phosphine product. Any trace of a signal at $\delta_{P}-0.8 \mathrm{ppm}$ means that the reaction is incomplete and must be allowed to react further. The removal of $\mathrm{P}(2 \text {-pyr })_{3}$ from ${ }^{2-p y r} \mathrm{PNP}$ is extremely difficult. 


\subsubsection{Step 2}

The second step of the reaction is to quench the nucleophilic pyridyl anion before the electrophile is added. Otherwise, this anion may compete with $\mathrm{P}(2-\mathrm{pyr})_{2}{ }^{-}$in subsequent reactions. Usually, this is achieved by adding tert-butyl chloride; an E1 reaction with $\mathrm{Li}(2$-pyr) produces 2-methylpropene, pyridine, and lithium chloride as byproducts. ${ }^{65}$ These are generally regarded as unreactive, and there is no evidence in the pyridylphosphine literature for any of these being responsible for the formation of intractable impurities. Nevertheless, after success in decreasing the reaction time, this step warranted further investigation.

Small aliquots of freshly prepared $\mathrm{KP}(2-\mathrm{pyr})_{2} / \mathrm{K}(2$-pyr $)$ in dimethoxyethane were combined with water, $\mathrm{NH}_{4} \mathrm{Cl}$, or tert-butyl chloride. The reactions were each dried under reduced pressure and the residues taken up in $\mathrm{CDCl}_{3}$. Figure 2.4 shows the resulting ${ }^{1} \mathrm{H}$ NMR spectra. The ${ }^{31} \mathrm{P}\left\{{ }^{1} \mathrm{H}\right\}$ NMR spectra of the reactions with water or $\mathrm{NH}_{4} \mathrm{Cl}$ were identical (singlet at $\delta_{P} 10 \mathrm{ppm}$ ); that of the tert-butyl chloride reaction contained more than one signal.

It is clear from Figure 2.4 that each reagent reacts differently with $\mathrm{KP}(2 \text {-pyr })_{2}$. In the ${ }^{1} \mathrm{H}$ NMR spectrum from the tert-butyl chloride reaction, the shape of the signal at $\delta_{H} 0.86 \mathrm{ppm}$ is very similar in shape to the major impurity highlighted in Figure 2.3, and is almost absent in the other two reactions. To confirm that tert-butyl chloride was responsible for the major impurity in the ligand synthesis reaction, $\mathrm{KP}(2 \text {-pyr })_{2} / \mathrm{K}\left(2\right.$-pyr) was reacted with $\mathrm{NH}_{4} \mathrm{Cl}$ then 2,6-bis(bromomethyl)pyridine. The impurity was no longer present. It may be have been due to 2 -methylpropene polymerisation involving the highly reactive pyridyl anion. 


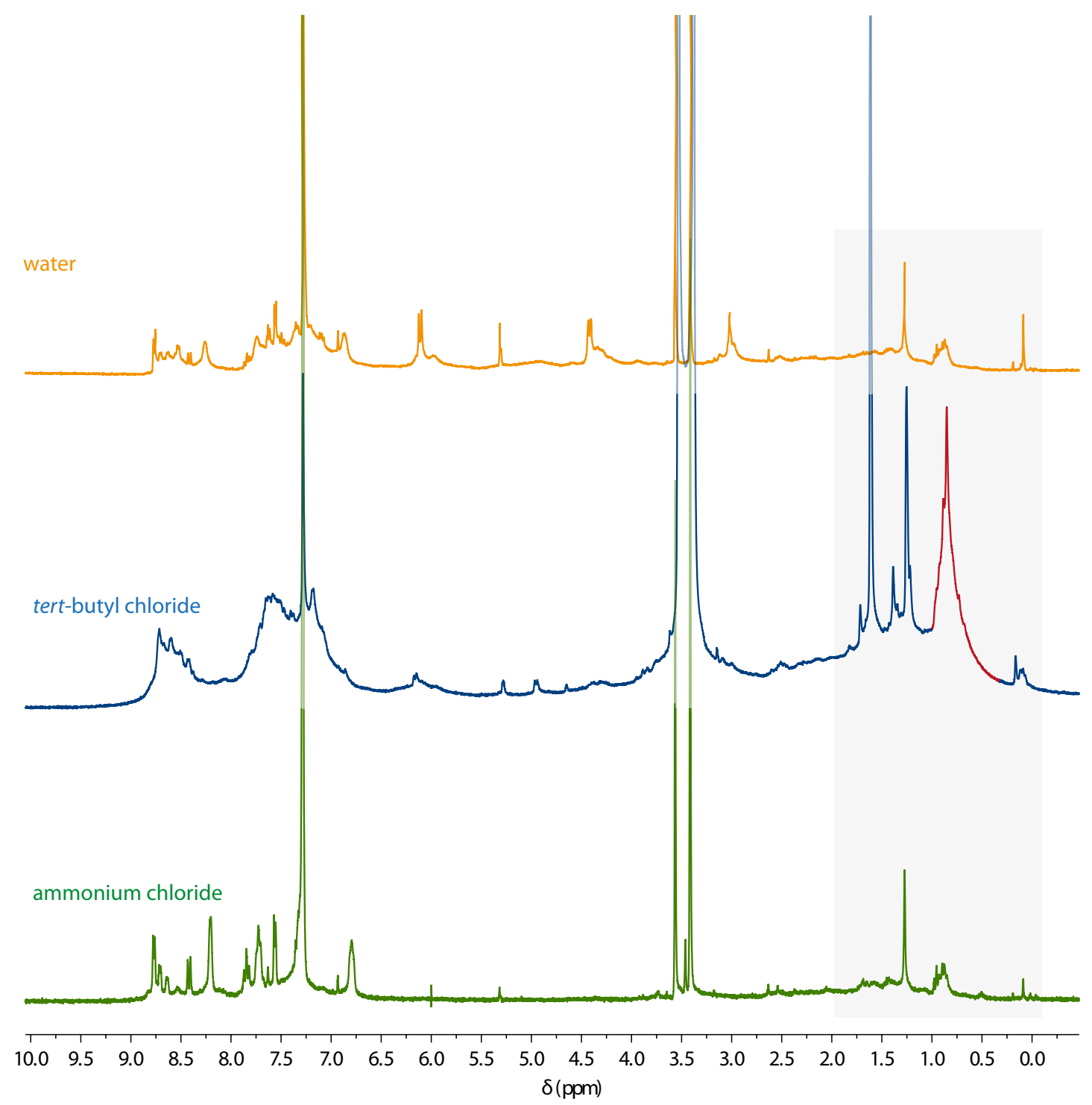

Figure 2.4 NMR spectra from the reactions of $\mathrm{KP}(2 \text {-pyr })_{2} / \mathrm{K}(2$-pyridyl $)$ with water, tert-butyl chloride, and ammonium chloride. Dimethoxyethane solvent: 3.4 and $3.6 \mathrm{ppm}$; tert-butyl chloride: $1.6 \mathrm{ppm}$. 
By using high sodium lithium powder and $\mathrm{NH}_{4} \mathrm{Cl}$ instead of potassium and tert-butyl chloride, the purity of both ${ }^{2-p y r} \mathrm{PNP}$ and other related pyridyl diphosphines was greatly increased (Figure 2.5).

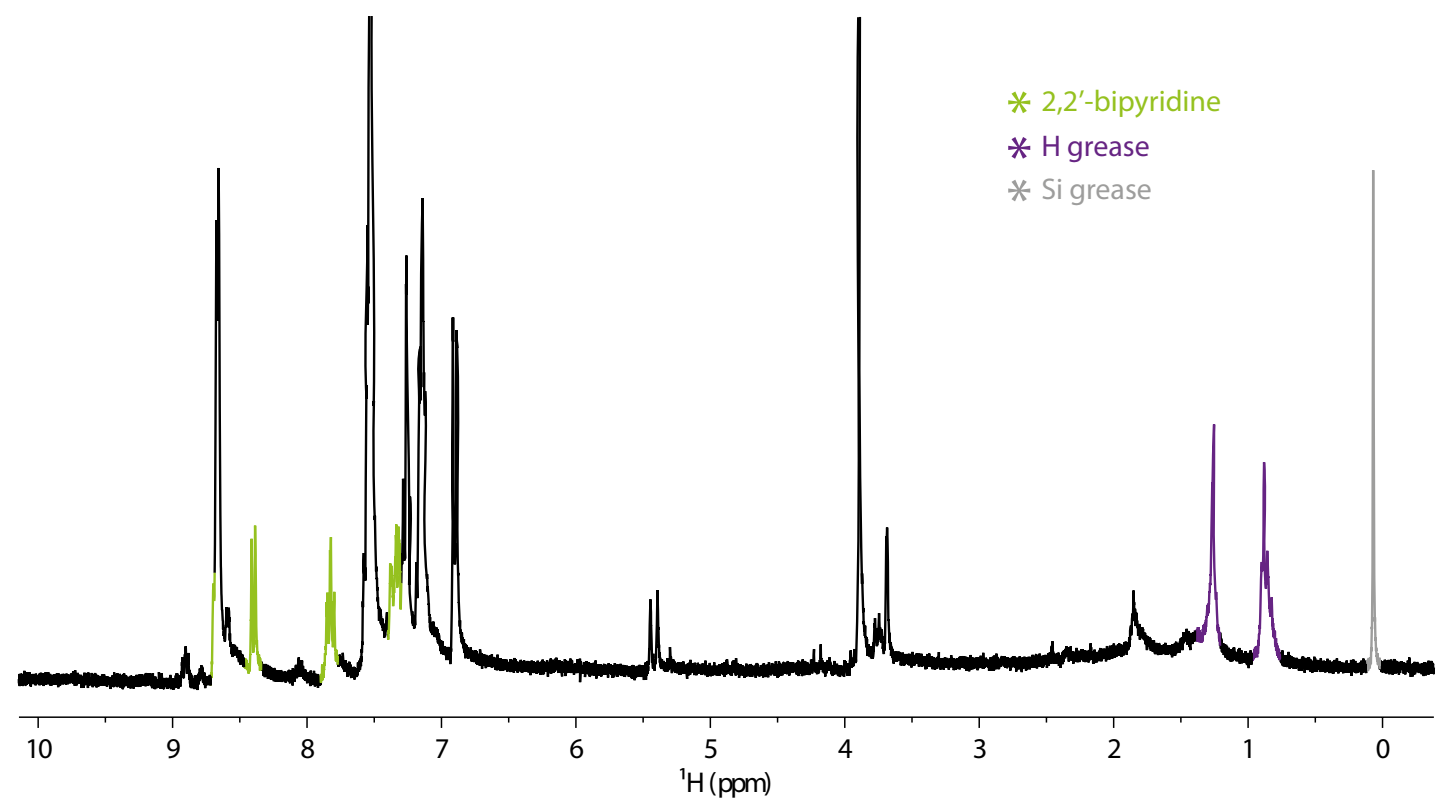

Figure 2.5 NMR spectrum of ${ }^{2-p y r} \mathrm{PNP}$ synthesis using lithium powder $/ \mathrm{NH}_{4} \mathrm{Cl}$.

\subsubsection{2,2'-Bipyridine}

The final hurdle in the purification of ${ }^{2-p y r} \mathrm{PNP}$ was the presence of 2,2'-bipyridine, which is highlighted in green in Figure 2.5. Unfortunately, it is an unavoidable byproduct of the $\mathrm{P}-\mathrm{C}_{2 \text {-pyr }}$ cleavage reaction. Uchida et al. have proposed that a reaction between 2-pyridyllithium and unreacted $\mathrm{P}(2 \text {-pyr })_{3}$ causes 2,2 '-bipyridine to be formed. This reaction is thought to pass through five-coordinate intermediate $\mathbf{2 3}$, which loses 2,2'-bipyridine through a ligand coupling process. ${ }^{105}$

2,2'-Bipyridine was removed from ${ }^{2-p y r} \mathrm{PNP}$ by sublimation, resulting in a pure sample. The ${ }^{1} \mathrm{H}$ NMR spectrum shown in Figure 2.6 is the result of the optimised synthesis shown in Scheme 2.12. 

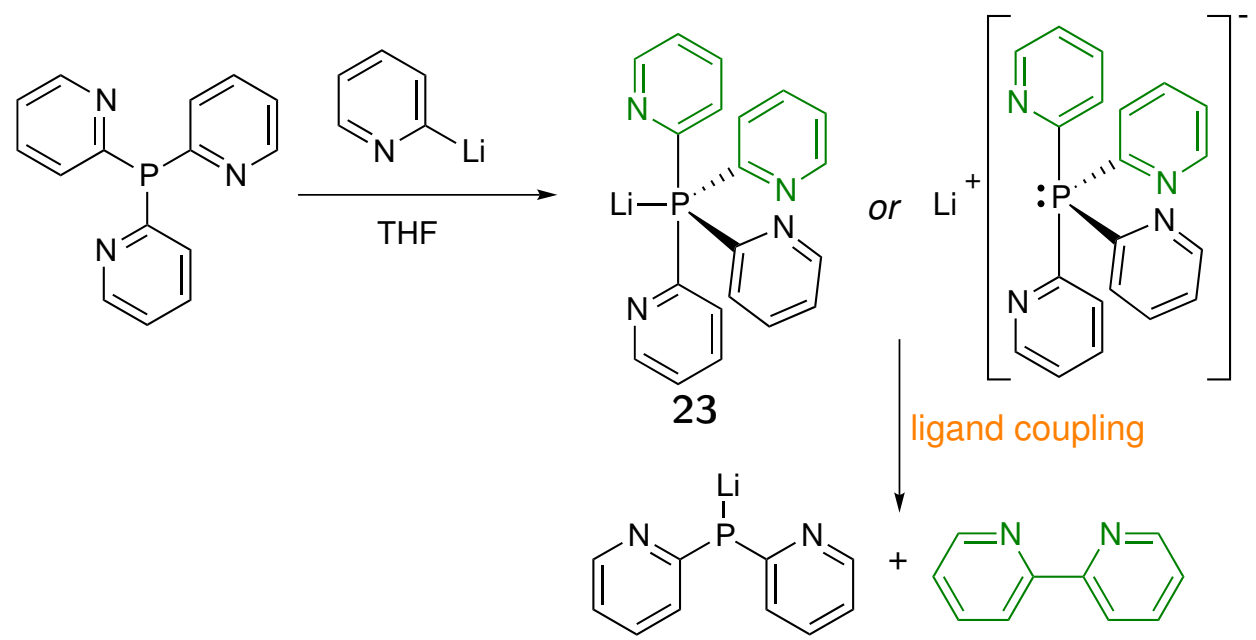

Scheme 2.11 Ligand coupling from a five-coordinate intermediate. ${ }^{105}$<smiles>c1ccc(P(c2ccccn2)c2ccccn2)nc1</smiles>

THF, $-78^{\circ} \mathrm{C}$ to rt, $1 \mathrm{~h}$

2) $\mathrm{NH}_{4} \mathrm{Cl},-78^{\circ} \mathrm{C}$ to rt, $30 \mathrm{~min}$<smiles>ClP(c1ccccn1)c1ccccn1</smiles><smiles>BrCc1cccc(CBr)n1</smiles>

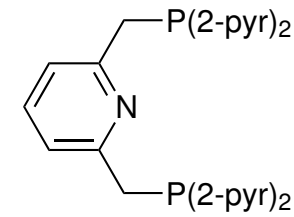

$78 \%$

Scheme 2.12 Optimised synthesis of ${ }^{2-p y r}$ PNP.

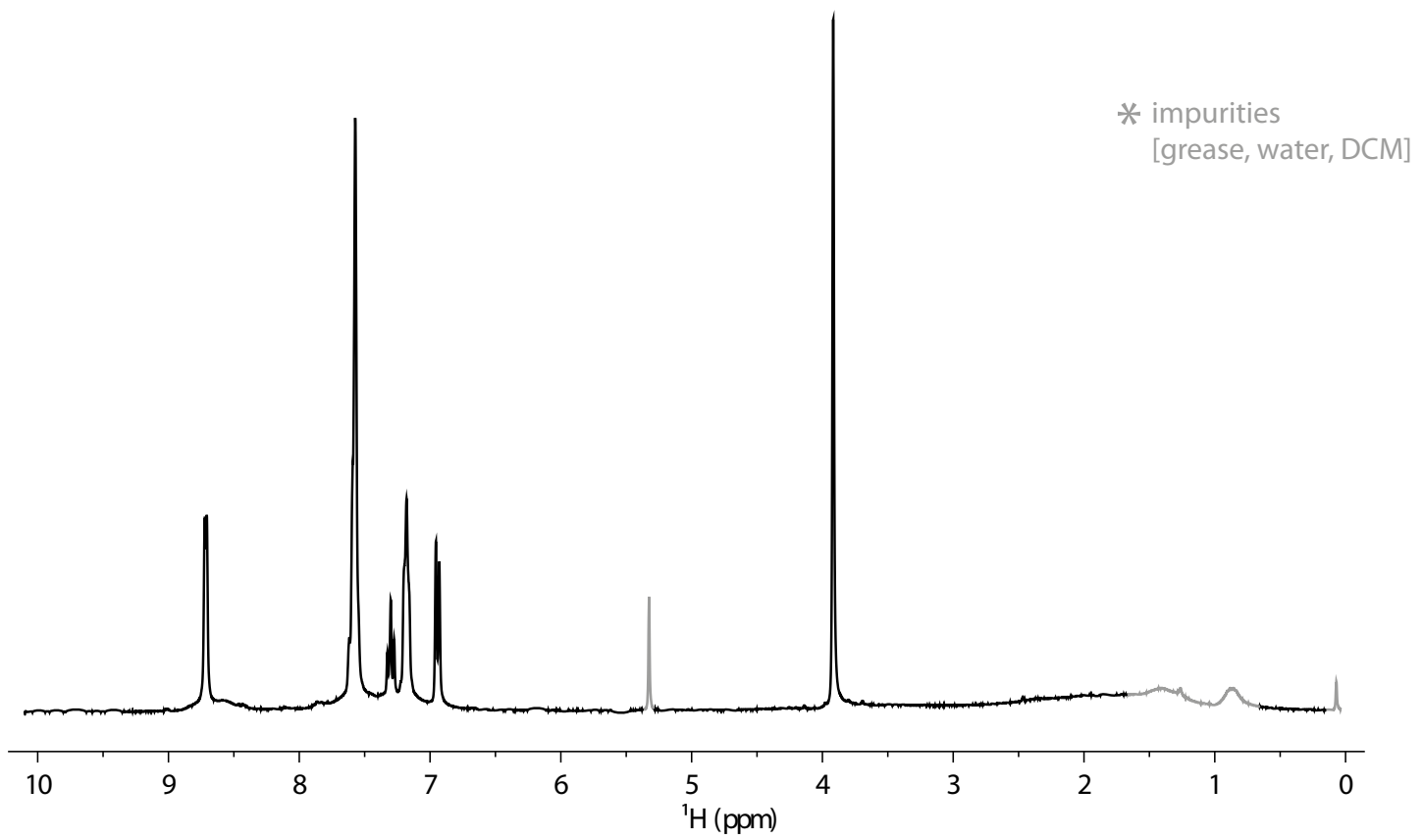

Figure 2.6 $\quad{ }^{1} \mathrm{H}$ NMR spectrum of ${ }^{2-\mathrm{pyr}} \mathrm{PNP}$. 


\subsubsection{Phosphorus(V)}

A route to ${ }^{2-p y r} \mathrm{PNP}$ via the phosphorus(V) oxide form $\mathbf{2 5}$ was also developed. It is convenient to have the option of synthesising an air-stable "pro-ligand", both for the ease of workup of an air-stable solid, and for long-term storage purposes. Tertiary phosphine oxides are often synthesised by coupling a secondary phosphine oxide with an alkyl halide. Secondary phosphine oxides are acidic, so can be deprotonated and reacted with electrophiles to form $\mathrm{P}-\mathrm{C}$ bonds.

The synthesis of bis(2-pyridyl)phosphine oxide (24) was a straightforward reaction of 2-pyridylmagnesium bromide with diethyl phosphite (Scheme 2.13). Diethyl phosphite exists in equilibrium with the phosphorus(III) acid form. If a base such as a Grignard reagent is added, it is deprotonated and remains in the acid form. The workup of $\mathbf{2 4}$ differs from that of a standard Grignard reaction in that only water is used to quench any remaining Grignard reagent. The addition of dilute acid results in the formation of 2,2-bipyridine through a mechanism related to that shown in Scheme 2.11. ${ }^{106}$

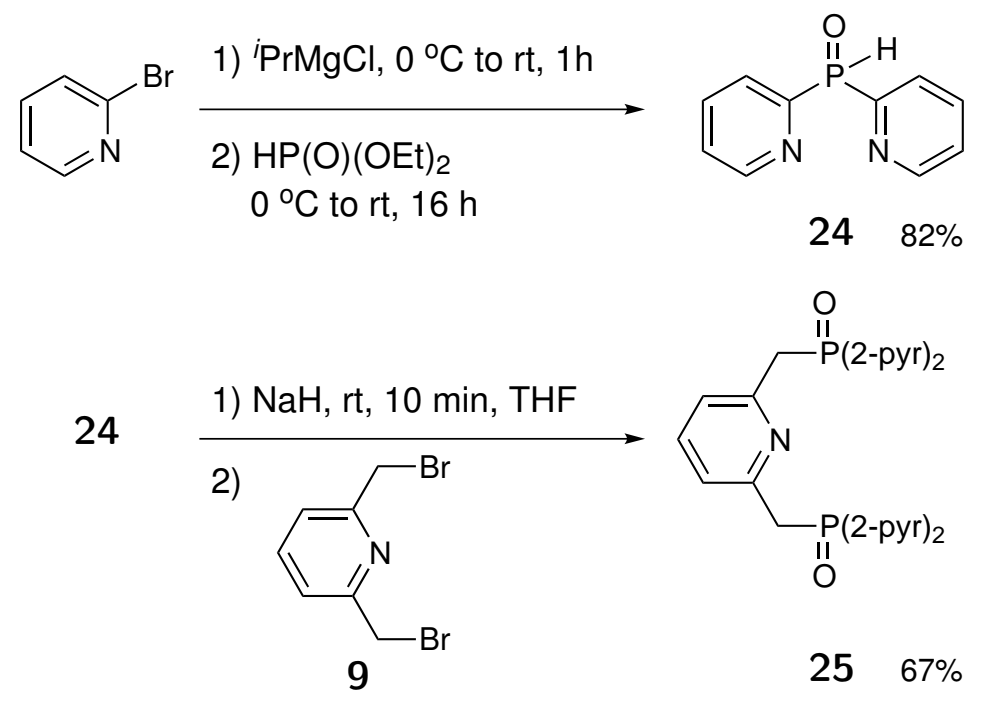

Scheme 2.13 Synthesis of ${ }^{2-p y r}$ PNP-oxide (25).

Compound 24 was deprotonated with sodium hydride and reacted with $\mathbf{9}$ in THF. This reaction was fast and gave $\mathbf{2 5}$ in high purity. In fact, $\mathbf{2 5}$ precipitated out of solution almost immediately upon addition of the backbone.

Phosphine oxides bearing pyridyl groups have been reduced by a mixture of trichlorosilane and triethyl amine in either refluxing xylenes or acetonitrile. ${ }^{107,108}$ Successful reduction of $\mathbf{2 5}$ was achieved in acetonitrile. In contrast, when $m$-xylene was used as the solvent $\mathrm{HP}(2-\mathrm{pyr})_{2}$ was isolated as the major product.

The purity of the reduced product was estimated to be $85 \%$ by NMR. Workup of 
the reduced phosphine was not optimised, as the phosphorus(III) route (vide supra) was extremely successful.

\subsubsection{Characterisation of 2-pyridylphosphines}

The ${ }^{31} \mathrm{P}\left\{{ }^{1} \mathrm{H}\right\}$ NMR spectra of ${ }^{2-p y r} \mathrm{PNP}$ and ${ }^{2-p y r} \mathrm{PNP}^{-\mathrm{O}_{2}}(\mathbf{2 5})$ display singlets at $\delta_{P}$ -6.3 and $26.3 \mathrm{ppm}$, respectively. Both resonances are present when ${ }^{2-p y r} \mathrm{PNP}$ has been exposed to air, either in solution or with the solvent removed. Thus, it is likely that both phosphorus atoms are oxidised very rapidly. This differs from the analogous PCP ligand, which undergoes stepwise oxidation and is only fully oxidised after 15-25 days. ${ }^{103}$ The ${ }^{2-p y r} \mathrm{PNP}$ ligand is extremely air-sensitive, so elemental analysis was not obtained. Infrared spectroscopy and mass spectrometry were carried out on $\mathbf{2 5}$. The NMR chemical shifts of both ${ }^{2-p y r} \mathrm{PNP}$ and $\mathbf{2 5}$ are summarised in Table 2.1. In the ${ }^{1} \mathrm{H}$ NMR spectrum of ${ }^{2-p y r} \mathrm{PNP}$, the $\mathrm{P}-\mathrm{CH}_{2}$ group appears a singlet. However, in the ${ }^{1} \mathrm{H}$ NMR spectrum of $\mathbf{2 5}$ this signal is a doublet due to coupling to phosphorus $\left({ }^{2} J_{\mathrm{P}-\mathrm{H}}=15.1 \mathrm{~Hz}\right)$.

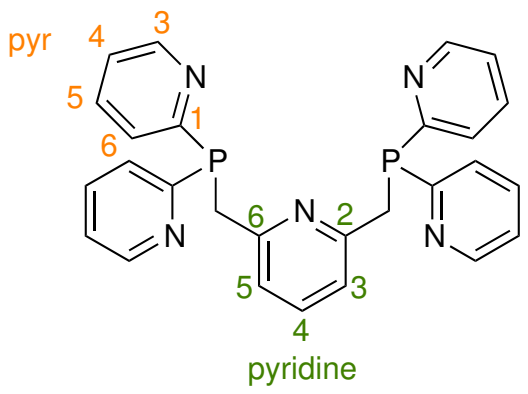

Figure 2.7 The numbering scheme for ${ }^{2-\mathrm{pyr}} \mathrm{PNP}$ ligand and ${ }^{2-\mathrm{pyr}} \mathrm{PNP}$-containing metal complexes is based on that of substituted pyridines.

Table 2.1 Selected ${ }^{1} \mathrm{H}$ and ${ }^{13} \mathrm{C}$ NMR data of ${ }^{2-p y r} \mathrm{PNP}$.

\begin{tabular}{llllll}
$\delta_{H} / \mathbf{p p m}$ & $\mathbf{P}-\mathbf{C H}_{\mathbf{2}}$ & $\mathbf{3}$ & $\mathbf{4}$ & $\mathbf{5}$ & $\mathbf{6}$ \\
\hline 2-pyr $\mathbf{P N P}$ & 3.95 & 8.66 & 7.25 & 7.54 & 7.51 \\
${ }_{\text {2-pyr }} \mathbf{P N P}$-oxide & 4.05 & 8.68 & 7.62 & 7.98 & 7.85
\end{tabular}

\begin{tabular}{llllll}
$\delta_{C} / \mathbf{p p m}\left({ }^{n} J_{\mathbf{P - C}}\right)$ & $\mathbf{P}-\mathbf{C H}_{\mathbf{2}}$ & $\mathbf{3}$ & $\mathbf{4}$ & $\mathbf{5}$ & $\mathbf{6}$ \\
\hline 2-pyr $\mathbf{P N P}$ & $35.8(13.8)$ & $150.1(8.6)$ & $122.5(\mathrm{~s})$ & $135.5(5.4)$ & $129.0(25.6)$ \\
${ }^{\text {2-pyr}} \mathbf{P N P}-\mathbf{O}_{\mathbf{2}}$ & $36.1(65.5)$ & $150.5(19.1)$ & $127.3(3.1)$ & $137.7(9.5)$ & $128.5(20.4)$ \\
\hline
\end{tabular}




\subsection{Synthesis of ${ }^{\mathrm{Ph}} \mathrm{PNP}$}

${ }^{\mathrm{Ph}} \mathrm{PNP}$ has similar steric and electronic properties to ${ }^{2-\mathrm{pyr}} \mathrm{PNP}$ (vide infra), but lacks the pyridyl nitrogens that provide ${ }^{2-p y r} \mathrm{PNP}$ with the ability to undergo hydrogen bonding interactions. Thus, ${ }^{\mathrm{Ph}} \mathrm{PNP}$ was synthesised in order to compare the chemistry of metal complexes with and without this ability. The ${ }^{\mathrm{Ph}} \mathrm{PNP}$ ligand was first reported in 1971, and its coordination chemistry with rhodium been studied extensively. ${ }^{109-112}$ It is usually synthesised using diphenyl phosphide, which is derived from diphenyl phosphine (Scheme 2.14). This is a flammable, air-sensitive, and foul-smelling liquid. Although it was available in the laboratory and could have been deprotonated and reacted with the appropriate electrophile (Scheme 2.14-A), an alternative route was employed.

The straightforward and high-yielding synthesis of ${ }^{2-p y r} \mathrm{PNP}$ from $\mathrm{P}(2 \text {-pyr })_{3}$ that was developed during this project was used to synthesise ${ }^{\mathrm{Ph}} \mathrm{PNP}$ from $\mathrm{PPh}_{3}$ (an air-stable and not particularly odorous solid). As was the case in the synthesis of ${ }^{2-p y r} \mathrm{PNP}$ the use of lithium powder meant that the cleavage of the $\mathrm{P}-\mathrm{Ph}$ bond was complete within 1 hour (Scheme 2.14-B). Pure ${ }^{\mathrm{Ph}} \mathrm{PNP}$ was obtained in $85 \%$ yield, a dramatic improvement over the $53 \%$ reported in the literature. ${ }^{78}$ Whereas ${ }^{2-p y r} \mathrm{PNP}$ is a bright yellow oil, ${ }^{\mathrm{Ph} P N P}$ is a white solid: the pyridyl nitrogens have a dramatic effect on the physical properties of the ligand.

A

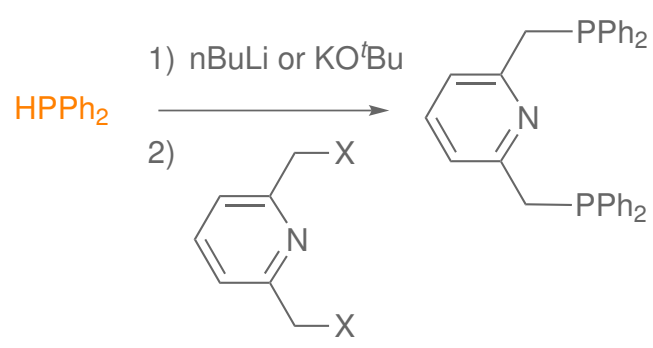

B
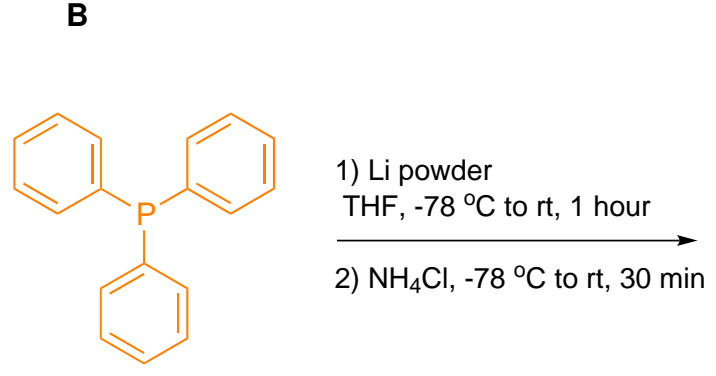

1) Li powder THF, $-78{ }^{\circ} \mathrm{C}$ to rt, 1 hour

2) $\mathrm{NH}_{4} \mathrm{Cl},-78^{\circ} \mathrm{C}$ to rt, $30 \mathrm{~min}$
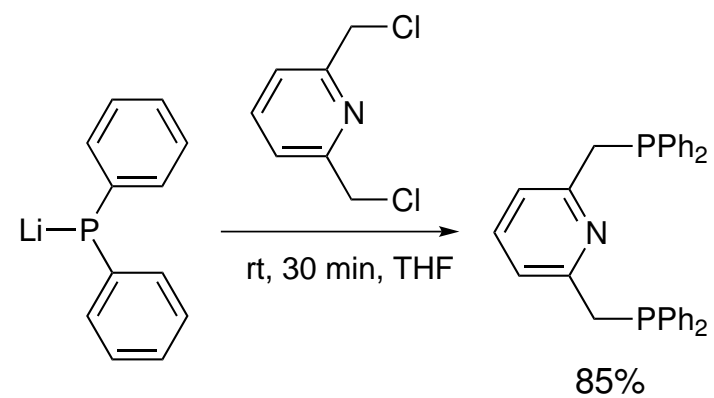

Scheme 2.14 Synthesis of PhPNP.A = literature method., ${ }^{78} \mathrm{~B}=$ method developed in this work. 


\subsection{Phosphine Selenides}

The steric and electronic properties of phosphine ligands dictate to a large extent the properties of the resulting metal complexes. Common methods used to quantify these properties include the "cone angle", used to approximate the steric influence of the substituents, and the $\mathrm{C}=\mathrm{O}$ stretching frequencies of $\left[\mathrm{Ni}(\mathrm{L})(\mathrm{CO})_{3}\right]$ complexes (where $\mathrm{L}$ is the ligand of interest), used to quantify electronic properties. ${ }^{113}$

Phosphine selenides have also been used to determine the effect of different substituents on the electronic properties of phosphine ligands. ${ }^{114}$ Selenium-77 is a spin-1/2 nucleus with a natural abundance of $7.63 \%$; the presence of $\mathrm{P}=$ Se bonds is visible in ${ }^{31} \mathrm{P}\left\{{ }^{1} \mathrm{H}\right\}$ NMR spectra as ${ }^{77}$ Se satellites on either side of the main signal. The magnitude of ${ }^{1} J_{\mathrm{P}-\mathrm{Se}}$ is indicative of the s-character of the phosphorus atoms and therefore the amount of $\sigma$-donation that these atoms can provide. This provides a way of measuring the effect of the substituents on the electronic properties of the phosphorus atoms, and is often much more straightforward than synthesising a carbonyl complex.

Phosphine selenides are synthesised in generally high yields by reacting phosphines with grey selenium. ${ }^{114}$ No selenide analogues of any PNP or PCP ligands have been reported. All three PNP ligands synthesised in this work ( ${ }^{\mathrm{Ph}} \mathrm{PNP},{ }^{t} \mathrm{Bu} P \mathrm{PP}$, and ${ }^{2-p y r} \mathrm{PNP}$ ) were reacted with selenium in order to determine ${ }^{1} J_{\mathrm{P}-\mathrm{Se}}$. The diselenide analogues ${ }^{2-p y r} \mathrm{PNP}-\mathrm{Se}_{2}(\mathbf{2 6}),{ }^{\mathrm{Ph}} \mathrm{PNP}-\mathrm{Se}_{2}(\mathbf{2 7})$, and ${ }^{t} \mathrm{Bu} \mathrm{PNP}_{-} \mathrm{Se}_{2}(\mathbf{2 8})$ were isolated in good yields and characterised by HRMS and NMR. Compounds $\mathbf{2 7}$ and $\mathbf{2 8}$ were characterised by elemental analysis. Surprisingly, although $\mathbf{2 7}$ and $\mathbf{2 8}$ are air-stable, pyridylphosphine selenide $\mathbf{2 6}$ is not. A bright red solid, insoluble in organic solvents, deposited as a fine layer inside glassware containing 26. This occurred to a large extent near the top of the solvent, suggesting that the oxygen diffusing into the solution is responsible.

It is likely that the solid is red selenium due to its insolubility, colour, and unique odour. Trioctylphosphine selenide is a common precursor to selenium-containing nanoparticles, and there are reports of secondary phosphine selenides decomposing to give red selenium. ${ }^{115,116}$ To confirm this would require analysis by atomic absorption spectroscopy and mass spectrometry, or inductively coupled plasma mass spectrometry. A simple flame test may show a blue colour (and give off a radish-like odour! ${ }^{117}$ ), but this could be a false positive if the decomposition product also contains selenium.

${ }^{31} \mathrm{P}\left\{{ }^{1} \mathrm{H}\right\}$ NMR data are displayed in Table 2.2. The value of ${ }^{1} J_{\mathrm{P}-\mathrm{Se}}$ for 26 is $742 \mathrm{~Hz}$. This is identical to that of ${ }^{2-p y r} \mathrm{PCP}$ and suggests that the pyridine backbone does not 


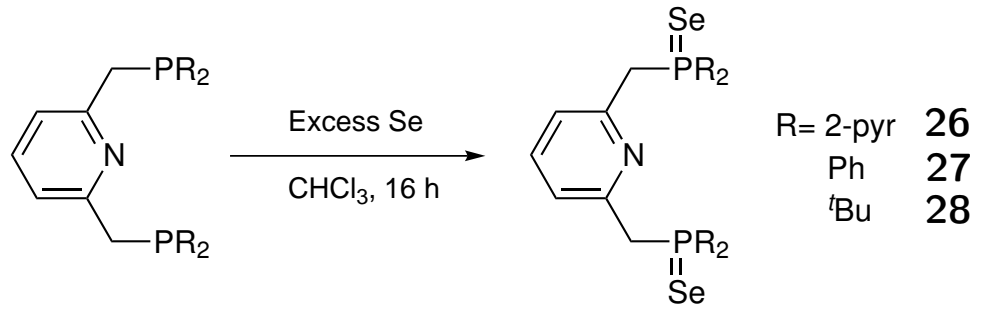

Scheme 2.15 Synthesis of phosphine selenides.

affect the electronic properties of the phosphorus atoms. ${ }^{103}$ Both pyridyl diphosphines have larger values of ${ }^{1} J_{\mathrm{P} \text {-Se }}$ than the phenyl analogue $(732 \mathrm{~Hz})$, suggesting that the pyridyl substituents are slightly more electron-withdrawing than phenyl groups. ${ }^{118}$ These data serve to confirm that, apart from the obvious steric difference, pyridyl substituents are more comparable to phenyl substituents with respect to their electronic properties than are tert-butyl substituents.

Table 2.2 ${ }^{31} \mathrm{P}\left\{{ }^{1} \mathrm{H}\right\}$ NMR data for phosphine selenides $\left(\mathrm{CDCl}_{3}\right.$ solvent). Data for ${ }^{2-\text { pyr }}$ PCP obtained by Vaughan. ${ }^{103}$

\begin{tabular}{lrr} 
Phosphine & ${ }^{1} J_{\mathbf{P}-\mathbf{S e}} / \mathbf{H z}$ & $\delta_{P} / \mathbf{p p m}$ \\
\hline${ }^{2-\text { pyr } \mathrm{PNP}}$ & 742 & 32.8 \\
${ }^{2-\text { pyr }} \mathrm{PCP}$ & 742 & 34.9 \\
${ }^{\mathrm{Ph}} \mathrm{PNP}$ & 732 & 33.1 \\
${ }^{t} \mathrm{Bu} \mathrm{PNP}$ & 705 & 76.0 \\
\hline
\end{tabular}

\subsection{Concluding Remarks}

Two routes to a new (2-pyridyl)-substituted PNP pincer ligand have been developed. Both provide ${ }^{2-p y r} \mathrm{PNP}$ in dramatically improved purity compared to the original method. The improved $\mathrm{P}(2 \text {-pyr })_{3}$ route has been successfully repeated by Vaughan to synthesise ${ }^{2-p y r} \mathrm{PCP}$ and ortho- $\mathrm{C}_{6} \mathrm{H}_{4}\left(\mathrm{CH}_{2} \mathrm{P}(2-\mathrm{pyr})_{2}\right)_{2} \cdot{ }^{103}$ It has also been used to synthesise ${ }^{\mathrm{Ph}} \mathrm{PNP}$ from a cheaper and easier-to-handle starting material in a higher yield than the literature report. ${ }^{78}$

Using high sodium lithium powder to cleave the $\mathrm{P}-\mathrm{C}$ bonds (rather than introducing the alkali metal as pieces, foil, or granules) considerably decreases the reaction time. This reduces the likelihood of organoalkali compounds reacting with the solvent and plays a role in improving the purity of ${ }^{2-p y r} \mathrm{PNP}$. It is likely that other new arylor heteroaryl-substituted PNP ligands could be successfully synthesised using this methodology. 
A fourth route to the known ligand ${ }^{t}$ BupNP has also been developed. Advantages over current methods include avoiding the use of the expensive and flammable reagent ${ }^{t} \mathrm{Bu}_{2} \mathrm{PH}$, and a much shorter reaction time.

Three new phosphine selenides were synthesised in order to determine the effect of 2-pyridyl substituents on the basicity of the phosphorus atoms. The 2-pyridyl substituents are more electron-withdrawing than phenyl substituents.

\subsection{Future Work}

Berners-Price et al. made a significant contribution to the pyridylphosphine literature when they reported the synthesis of 1,2-bis(di-2-pyridylphosphino)ethane from $\mathrm{P}(2$ pyr $)_{3}$ and slivers of lithium metal. ${ }^{65}$ Previous syntheses required the appropriate chlorophosphine $\left(\mathrm{RPCl}_{2}\right)$, which can be both difficult and expensive to synthesise.

However, the success of this method did not extend to the analogous 3- and 4pyridylphosphines. Berners-Price et al. were unable to use lithium to cleave a 3- or 4-pyridyl group from the corresponding tris(pyridyl)phosphine; instead, they were only able to synthesise 3- and 4-pyridylphosphines from bromopyridine and $n$-BuLi/TMEDA. This method remains the only successful route to such ligands and has only been used to synthesise two. ${ }^{65,119}$

As discussed in subsection 2.3.1, the methods established by Berners-Price et al. did not yield pure ${ }^{2-p y r} \mathrm{PNP},{ }^{2-\text { pyr }} \mathrm{PCP}$, or ortho- $\mathrm{C}_{6} \mathrm{H}_{4}\left(\mathrm{CH}_{2} \mathrm{P}(2-\mathrm{pyr})_{2}\right)_{2}{ }^{103} \mathrm{~A}$ new method was developed in this work and resulted in very pure samples of all three pyridylphosphines. Thus, it would be very interesting to establish whether this method would allow the analogous 3- and 4-pyridylphosphines to be synthesised. These confer greater water solubility on metal complexes than do 2-pyridyl groups. ${ }^{62}$ 


\section{Chapter 3}

\section{Rhodium Complexes}

Rhodium complexes are used to catalyse a number of important reactions, including the hydrogenation of alkenes and the industrial scale hydroformylation of propene under biphasic conditions. ${ }^{120,121}$ Although rhodium pincer complexes have not been reported to catalyse the hydration of unsaturated substrates, it is not difficult to find reports describing the oxidative addition of water to $\mathrm{Rh}(\mathrm{I})$, or reports of $\mathrm{Rh}-\mathrm{O}$ bonds. These are both likely to be elements of a catalytic hydrogenation reaction. Furthermore, the ability of $\left[\left({ }^{\mathrm{Ph} P N P}\right) \mathrm{Rh}\left(\mathrm{C}_{2} \mathrm{H}_{4}\right)\right] \mathrm{BF}_{4}$ to be converted to a styrene complex at room temperature is particularly promising from the point of view of catalytic hydration, as it demonstrates that a rhodium pincer complex can undergo an important elementary step in this reaction - the coordination of a substrate to the metal. ${ }^{122}$

Synthesising rhodium complexes is an excellent starting point when studying the coordination chemistry of a novel ligand, as ${ }^{103} \mathrm{Rh}$ is a $100 \%$ abundant spin $1 / 2$ nucleus. This means that the NMR spectra of rhodium complexes can provide additional information about the nature of any complexes or intermediates formed. For complexes bearing phosphine ligands, the magnitude of ${ }^{1} J_{\mathrm{Rh}-\mathrm{P}}$ is particularly useful, as it provides information about the oxidation state and coordination number of the rhodium centre.

This chapter describes a comparison between the rhodium coordination chemistry of 2-pyrPNP and that of ${ }^{\mathrm{Ph}} \mathrm{PNP},{ }^{i}{ }^{\mathrm{Pr}} \mathrm{PNP}$, and ${ }^{{ }^{\mathrm{B}} \mathrm{Bu}} \mathrm{PNP}$. Section 3.1 summarises attempts to synthesise a rhodium(I) chlorido complex, and section 3.2 describes attempts to synthesise a cationic cyclooctene complex and a neutral triflate complex. 


\subsection{Reactions of ${ }^{2-p y r} \mathrm{PNP}$ with $\left[\mathrm{Rh}(\operatorname{coe})_{2}(\mu-\mathrm{Cl})\right]_{2}$ and $[\mathrm{Rh}(\operatorname{cod})(\mu-\mathrm{Cl})]_{2}$}

Although it is important to compare the chemistry of ${ }^{2-p y r} \mathrm{PNP}$ with that of the wellstudied ligand ${ }^{t} \mathrm{Bu} P N P$, rhodium(I) complexes of ${ }^{\mathrm{Ph}} \mathrm{PNP}$ should be more comparable to those of ${ }^{2-p y r} \mathrm{PNP}$ in terms of the steric and electronic demands of the pincer ligand. The first example of a rhodium(I) ${ }^{\mathrm{Ph}} \mathrm{PNP}$ complex was reported in 1981 by Sacco et al. ${ }^{123}$ The rhodium(I) chlorido complex [( $\left.\left.{ }^{\mathrm{Ph} P N P}\right) \mathrm{RhCl}\right](\mathbf{2 9})$ activated a number of small molecules including $\mathrm{H}_{2}, \mathrm{O}_{2}$, and $\mathrm{CO}_{2} \cdot{ }^{123}$

In attempts to synthesise the ${ }^{2-p y r} \mathrm{PNP}$ analogue of $\mathbf{2 9},{ }^{2-p y r} \mathrm{PNP}$ was reacted with both $\left[\mathrm{Rh}(\operatorname{coe})_{2}(\mu-\mathrm{Cl})\right]_{2}$ and $[\mathrm{Rh}(\operatorname{cod})(\mu-\mathrm{Cl})]_{2}$ in $\mathrm{CDCl}_{3}$. Sacco et al. carried out the reaction of ${ }^{\mathrm{Ph}} \mathrm{PNP}$ with $\left[\mathrm{Rh}(\mathrm{coe})_{2}(\mu-\mathrm{Cl})\right]_{2}$ in benzene; however, initial reactions were carried out in chloroform to ensure solubility of the ligand. On reaction of ${ }^{2-p y r} \mathrm{PNP}$ with $\left[\mathrm{Rh}(\mathrm{coe})_{2}(\mu-\mathrm{Cl})\right]_{2}$, the chloroform solution turned dark brown immediately. The sample was analysed by NMR the next day, by which time a dark solid had settled to the bottom of the NMR tube (Scheme 3.1). NMR analysis of the clear red chloroform solution showed the presence of two rhodium complexes. In the ${ }^{31} \mathrm{P}\left\{{ }^{1} \mathrm{H}\right\} \mathrm{NMR}$ spectrum, the signal for the free ligand was absent and two doublets at $\delta_{P} 30.7$ and $33.4 \mathrm{ppm}$ had appeared $\left({ }^{1} J_{\mathrm{Rh}-\mathrm{P}}=91 \mathrm{~Hz}\right.$ and $105 \mathrm{~Hz}$, respectively). These coupling constants indicate that the rhodium centre has been oxidised to rhodium(III) in both cases. One-bond coupling constants, such as ${ }^{1} J_{\mathrm{Rh}-\mathrm{P}}$, are affected by the s-character of the bond, which is in turn affected by the oxidation state of the metal. Thus, the magnitude of ${ }^{1} J_{\mathrm{Rh}-\mathrm{P}}$ for a rhodium(I) complex ( $\mathrm{dsp}^{2}$ hybridisation) is larger than that of a rhodium(III) complex $\left(\mathrm{d}^{2} \mathrm{sp}^{3}\right) .{ }^{124}$
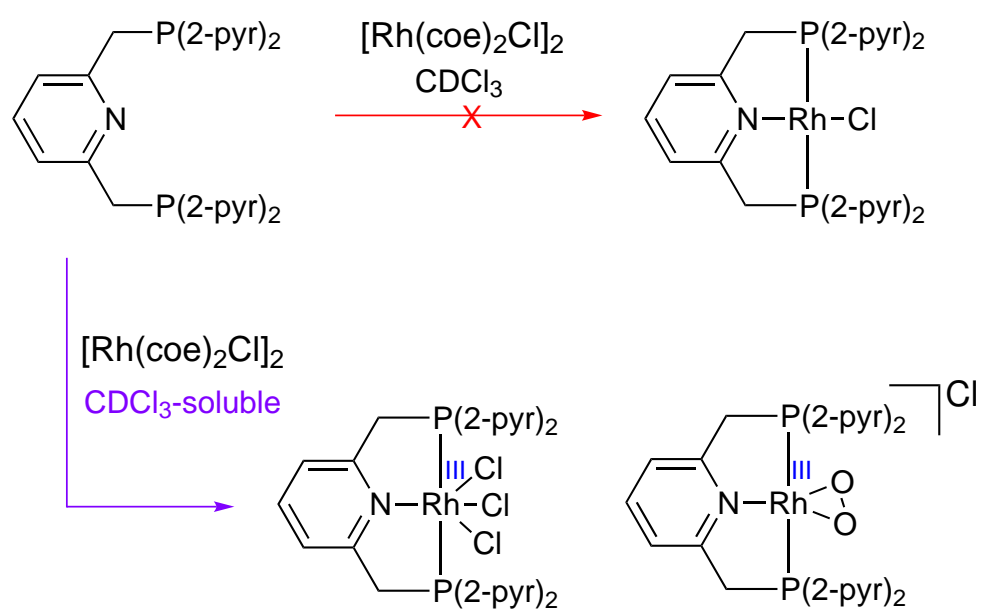

Scheme 3.1 Possible chloroform-soluble rhodium(III) complexes formed when ${ }^{2-p y r} \mathrm{PNP}$ was reacted with $\left[\mathrm{Rh}(\mathrm{coe})_{2} \mathrm{Cl}\right]_{2}$ in $\mathrm{CDCl}_{3}$.

The ${ }^{1} \mathrm{H}$ NMR spectra of the two complexes are very similar. The pyridyl protons 
appeared as four resonances in the ${ }^{1} \mathrm{H}$ NMR spectrum, which indicates that all four pyridyl rings are in the same environment. The appearance of the $\mathrm{P}-\mathrm{CH}_{2}$ resonances as virtual triplets means that the pincer ligand is coordinated in a meridional fashion - the trans phosphorus atoms are able to couple strongly to each other. In complexes where the pincer ligand is facially coordinated, the $\mathrm{P}-\mathrm{CH}_{2}$ protons appear as an $\mathrm{AB}$ system, and virtual coupling to the phosphorus atoms is absent (see chapter four).

The identities of the two chloroform-soluble rhodium(III) complexes were not determined, but possible structures are shown in Scheme 3.1. These are derived from oxidation by chloroform and reaction with adventitious oxygen, respectively. The ${ }^{\mathrm{Ph}} \mathrm{PNP}$ versions of similar complexes were reported in 1981 by Vasapollo et al., but NMR data are not available for comparison. The dioxygen complex was not very stable in solution. Vasapollo et al. noted that one of its decomposition products was oxidised ${ }^{\mathrm{Ph}} \mathrm{PNP} .{ }^{123}$

Reaction of ${ }^{2-p y r} \mathrm{PNP}$ with $[\mathrm{Rh}(\operatorname{cod})(\mu-\mathrm{Cl})]_{2}$ in $\mathrm{CDCl}_{3}$ gave a red solution, with a dark solid setting out over an hour. NMR analysis of the solution showed the presence of one major product $\left(\delta_{P} 28.3,{ }^{1} J_{\mathrm{Rh}-\mathrm{P}}=92 \mathrm{~Hz}\right)$. These data are similar to those observed for one of the chloroform-soluble rhodium(III) complexes obtained by reacting ${ }^{2-p y r} \mathrm{PNP}$ with $\left[\mathrm{Rh}(\mathrm{coe})_{2}(\mu-\mathrm{Cl})\right]_{2}$.

The rhodium complex was precipitated from chloroform by the addition of diethyl ether. The resultant tan solid was analysed by mass spectrometry and elemental analysis. High resolution mass spectrometry (HRMS) gave a good match for $\left[\left({ }^{2-p y r} \mathrm{PNP}\right) \mathrm{RhCl}_{3}\right]$ with the monoprotonated adduct $\mathrm{M}+\mathrm{H}^{+}$detected at $\mathrm{m} / z=$ $687.9718 \mathrm{amu}$. Elemental analysis of the complex was not in perfect agreement; however, the accuracies of the analyses obtained for other pyridyl phosphine complexes have also been affected by water (see chapter four). Recalculated with 1.5 equivalents of water, the values were in very good agreement with the HRMS data and taken together suggest that a rhodium(III) trichlorido complex had formed.

Vaughan has used chloroform as a solvent for reactions between rhodium(I) and ${ }^{2-p y r}$ PCP complexes without issue. ${ }^{103}$ However, these investigations focussed on coordinating rhodium(I) to the pyridyl nitrogens rather than on rhodium pincer chemistry. Pincer chemistry may be more sensitive to the use of chloroform as, for example, chlorinated solvents have been reported to deactivate rhodium hydrogenation catalysts. ${ }^{125}$

The solids that settled out of the two reactions with cyclooctene-ligated rhodium complexes were investigated. The solid from the reaction with $\left[\mathrm{Rh}(\operatorname{coe})_{2}(\mu-\mathrm{Cl})\right]_{2}$ 
dissolved in $\mathrm{D}_{2} \mathrm{O}$ and was insoluble in pyridine- $\mathrm{d}_{5}$, acetone- $\mathrm{d}_{6}$, and $\mathrm{CDCl}_{3}$, while the solid from the $[\mathrm{Rh}(\operatorname{cod})(\mu-\mathrm{Cl})]_{2}$ reaction was insoluble in all four solvents. An IR spectrum did not shed light on the identity of this solid.

NMR spectra of the solid from the $\left[\mathrm{Rh}(\operatorname{coe})_{2}(\mu-\mathrm{Cl})\right]_{2}$ reaction were recorded in $\mathrm{D}_{2} \mathrm{O}$. The broad signals in the ${ }^{1} \mathrm{H}$ NMR spectrum, combined with the absence of ${ }^{31} \mathrm{P}\left\{{ }^{1} \mathrm{H}\right\}$ NMR signals, suggest that the solution contains paramagnetic species. In order to investigate this hypothesis, the acquisition time of the ${ }^{31} \mathrm{P}\left\{{ }^{1} \mathrm{H}\right\}$ NMR experiment was decreased from 1 second to $0.05 \mathrm{~s}$, and the delay time (d1) was decreased from $1 \mathrm{~s}$ to $0.05 \mathrm{~s}$. A very broad peak centred on $50 \mathrm{ppm}$ appeared in the resulting ${ }^{31} \mathrm{P}\left\{{ }^{1} \mathrm{H}\right\} \mathrm{NMR}$ spectrum. These modified parameters increase the chance of observing any complexes containing quickly-relaxing nuclei and allow many more scans to be collected in a shorter time.

A plausible structure for the paramagnetic complex responsible for the broad spectrum is shown in Scheme 3.2. This is based on a report by Milstein et al. of the ${ }^{t}$ BupNP analogue (vide infra). ${ }^{126}$
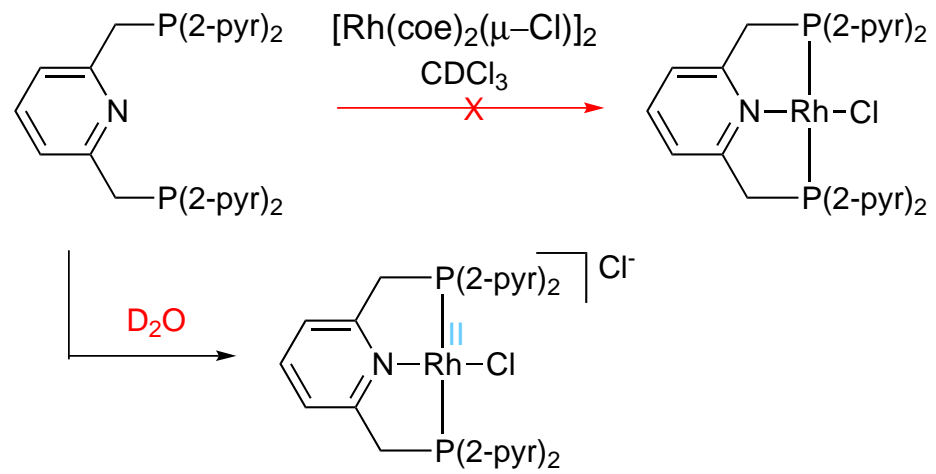

Scheme 3.2 Proposed $\mathrm{D}_{2} \mathrm{O}$-soluble product of the reaction of ${ }^{2-p y r} \mathrm{PNP}$ with $\left[\mathrm{Rh}(\mathrm{coe})_{2}(\mu-\mathrm{Cl})\right]_{2}$.

To prevent interference by chloroform, the reaction of $\left[\mathrm{Rh}(\text { coe })_{2}(\mu-\mathrm{Cl})\right]_{2}$ and ${ }^{2-p y r} \mathrm{PNP}$ was repeated in toluene. After 40 minutes of stirring at room temperature, the reaction mixture was dried and the tan solid dissolved in $\mathrm{CD}_{2} \mathrm{Cl}_{2}$ (Scheme 3.3). ${ }^{31} \mathrm{P}\left\{{ }^{1} \mathrm{H}\right\}$ NMR data suggested rhodium(I) complex [( $\left.{ }^{2-\mathrm{pyr}} \mathrm{PNP}\right) \mathrm{RhCl}$ had formed. Two doublets separated by $0.3 \mathrm{ppm}$ had rhodium-phosphorus coupling constants of $144 \mathrm{~Hz}$. This value of ${ }^{1} J_{\mathrm{Rh}-\mathrm{P}}$ is consistent with those reported for $\left[\left({ }^{\mathrm{Ph} P N P}\right) \mathrm{RhCl}\right]$ and $\left[\left({ }^{t} \mathrm{Bu} \mathrm{PNP}\right) \mathrm{RhCl}\right]\left({ }^{1} J_{\mathrm{Rh}-\mathrm{P}}=152\right.$ and $145 \mathrm{~Hz}$, respectively). ${ }^{79,111}$

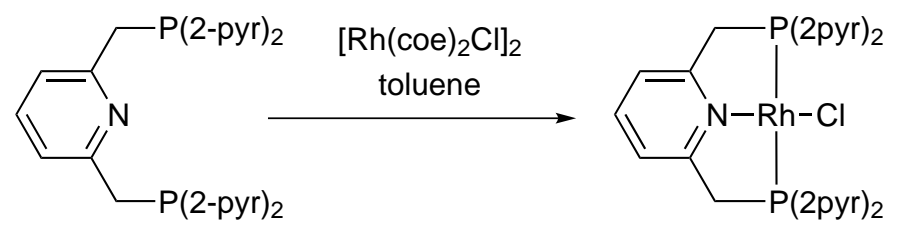

Scheme 3.3 Reaction of ${ }^{2-p y r} \mathrm{PNP}$ with $\left[\mathrm{Rh}(\operatorname{coe})_{2}(\mu-\mathrm{Cl})\right]_{2}$ in toluene. 
The ${ }^{31} \mathrm{P}\left\{{ }^{1} \mathrm{H}\right\}$ NMR spectrum also contained a number of other resonances, including a singlet due to a small amount of ${ }^{2-p y r}{ }^{2 N P}-\mathrm{O}_{2}$ and a doublet at $\delta_{P} 29.4 \mathrm{ppm}$ $\left({ }^{1} J_{\mathrm{Rh}-\mathrm{P}}=88 \mathrm{~Hz}\right)$. The formation of rhodium(III) complex $\left[\left({ }^{2-\mathrm{pyr}} \mathrm{PNP}\right) \mathrm{RhCl}_{3}\right]$ is likely to be responsible for this signal. The identities of the complexes responsible for the two resonances near $\delta_{P} 50 \mathrm{ppm}$ were not investigated.

The presence of two doublets in the ${ }^{31} \mathrm{P}\left\{{ }^{1} \mathrm{H}\right\}$ NMR spectrum with the same value of ${ }^{1} J_{\mathrm{Rh}-\mathrm{P}}$ may be due to deuterium incorporation at the $\mathrm{P}-\mathrm{CH}_{2}$ positions of the ligand. The presence of deuterium results in a slight shielding of ${ }^{31} \mathrm{P}\left\{{ }^{1} \mathrm{H}\right\} \mathrm{NMR}$ resonances. ${ }^{127}{ }^{2} \mathrm{H}$ NMR analysis of the reaction mixture is required. The $\mathrm{P}-\mathrm{CH}_{2}$ signal was broad and, because of the presence of a number of pyridyl-containing species, integration could not be used to determine whether deuterium incorporation had occurred.

\subsection{Reactions of ${ }^{2-p y r} \mathrm{PNP}$ with $\left.[\mathrm{Rh} \text { (acetone })_{2}(\text { coe })_{2}\right] \mathrm{PF}_{6}$ and $\left[\mathrm{Rh}(\text { coe })_{2}(\mu \text {-OTf })\right]_{2}$}

After discovering that ${ }^{2-p y r} \mathrm{PNP}$ reacts very differently to ${ }^{\mathrm{Ph}} \mathrm{PNP}$ and ${ }^{t} \mathrm{Bu} \mathrm{PNP}$ in reactions where rhodium(I) chlorido pincer complexes are usually obtained, the syntheses of ${ }^{2-p y r}$ PNP cyclooctene and triflate complexes were investigated.

The chlorido ligands of $\left[\mathrm{Rh}(\mathrm{coe})_{2}(\mu-\mathrm{Cl})\right]_{2}$ are easily removed with silver salts $(\mathrm{AgX})$ to form either a solvent-containing complex (in the case of X being a weakly coordinating counterion) or $\left[\mathrm{Rh}(\mathrm{coe})_{2}(\mu-\mathrm{X})\right]_{2} \cdot{ }^{128,129}$ Scheme 3.4 shows the products isolated when

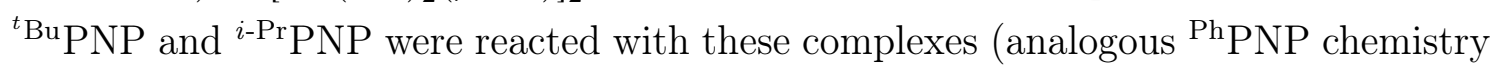
has not been reported). The cationic complexes $\left[\left({ }^{\mathrm{R}} \mathrm{PNP}\right) \mathrm{M}(\mathrm{coe})\right]^{+}$have been used as an entry point to chemistry involving metal-oxygen bonds. Thus, it was important to determine whether the analogous ${ }^{2-p y r}$ PNP complex could be synthesised.

First, an orange solution of $\left[\mathrm{Rh}(\text { acetone })_{2}(\mathrm{coe})_{2}\right] \mathrm{PF}_{6}$ in acetone* was reacted with ${ }^{2-p y r} \mathrm{PNP}$. This resulted in a dark red-brown solution. Addition of pentane precipitated a brown solid in near-quantitative yield. Unlike the ${ }^{t}$ Bu PNP complex, characterisation of the ${ }^{2-p y r} \mathrm{PNP}$-containing product by NMR spectroscopy was difficult. The ${ }^{1} \mathrm{H}$ NMR spectrum displayed a very broad pyridyl region whilst the solvent peaks were sharp. Furthermore, the ${ }^{31} \mathrm{P}\left\{{ }^{1} \mathrm{H}\right\}$ NMR spectrum was missing any signal belonging to the ligand, even though the $\mathrm{PF}_{6}{ }^{-}$septet remained sharp. Shortening the acquisition time

${ }^{*}\left[\mathrm{Rh}(\text { acetone })_{2}(\mathrm{coe})_{2}\right] \mathrm{PF}_{6}$ was synthesised from $\left[\mathrm{Rh}(\mathrm{coe})_{2} \mathrm{Cl}\right]_{2}$ and $\mathrm{AgPF}_{6}$ in acetone. $\mathrm{AgCl}$ was filtered off, but the $\left[\mathrm{Rh}(\text { acetone })_{2}(\mathrm{coe})_{2}\right] \mathrm{PF}_{6}$ was not isolated. ${ }^{129}$ 

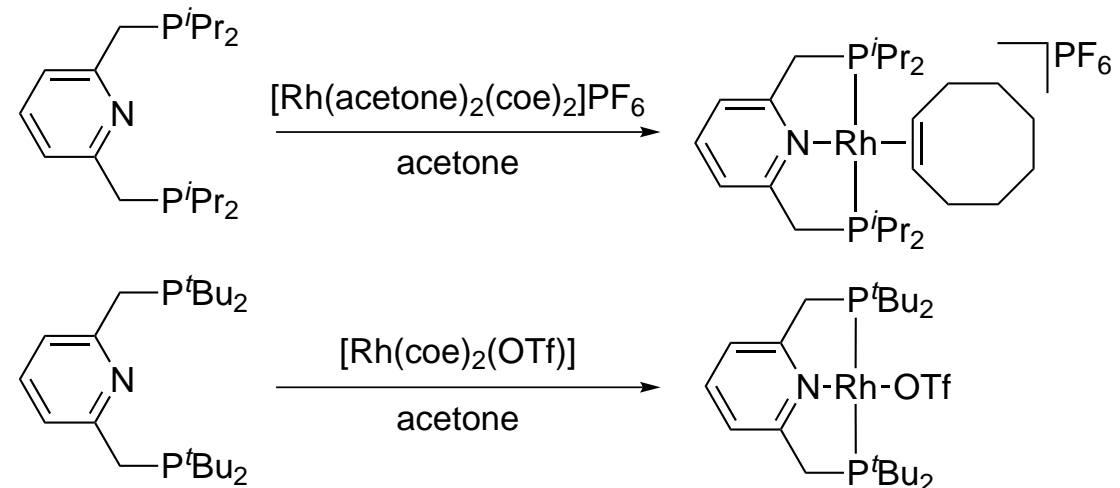

Scheme 3.4 Reactions of PNP ligands with rhodium(I) cyclooctene complexes. ${ }^{128,130}$

of the ${ }^{31} \mathrm{P}\left\{{ }^{1} \mathrm{H}\right\}$ NMR experiment resulted in the appearance of a broad signal $\left(\delta_{P}\right.$ $25 \mathrm{ppm}, \nu_{1 / 2}=353 \mathrm{~Hz}$ ). As the relaxation of the $\mathrm{PF}_{6}{ }^{-}$phosphorus nucleus was not affected to any great extent, this experiment strongly suggested that the broadening of the pyridyl signals was due to a local unpaired electron rather than a small amount of a rhodium(II) impurity. However, the NMR experiment cannot provide any information as to whether the unpaired electron is located on rhodium or the ligand backbone.

Similar spectra were obtained when ${ }^{2-p y r} \mathrm{PNP}$ was reacted with a THF solution of $\left[\mathrm{Rh}(\mathrm{coe})_{2}(\mu \text {-OTf })\right]_{2}$. This precursor was synthesised by abstracting the chlorido ligands from $\left[\mathrm{Rh}(\mathrm{coe})_{2} \mathrm{Cl}\right]_{2}$ using AgOTf, followed by filtration (Scheme 3.5). ${ }^{128}$ Triflate is able to bind weakly to rhodium(I) as THF is a more weakly-coordinating solvent than acetone. ${ }^{128}$ The ${ }^{19} \mathrm{~F}$ NMR resonance at $-74.0 \mathrm{ppm}$ is slightly out of the typical range for a weakly coordinated triflate anion $\left(\delta_{F}-77.0\right.$ to $\left.-78.0 \mathrm{ppm}\right)$. A similar shift to $\delta_{F}-73.4 \mathrm{ppm}$ was observed by Ozerov et al. after oxidising a rhodium(I) complex to rhodium(II) using AgOTf. ${ }^{131}$ The change in ${ }^{19} \mathrm{~F}$ NMR chemical shift was ascribed to the presence of a paramagnetic environment around the triflate anion. Broadening of the ${ }^{1} \mathrm{H}$ NMR spectrum and the absence of ${ }^{31} \mathrm{P}$ signals suggest that another paramagnetic ${ }^{2-p y r}$ PNP complex has been synthesised. EPR spectroscopy would confirm the number of unpaired electrons, and an X-ray crystal structure would be helpful in confirming (or denying) the presence of a square planar rhodium(II) centre. Unfortunately, access to an EPR spectrometer was not available, and crystals suitable for an X-ray diffraction study were not obtained.

In order to be more confident that the modified ${ }^{31} \mathrm{P}\left\{{ }^{1} \mathrm{H}\right\}$ NMR experiment was in fact detecting paramagnetic species, the spectra of the paramagnetic complex $\left[\left({ }^{t}{ }^{\mathrm{Bu}} \mathrm{PNP}\right) \mathrm{RhCl} \mathrm{BF}_{4}(\mathbf{3 0})\right.$ were collected using the same parameters and compared with the data from the reactions of ${ }^{2-p y r} \mathrm{PNP}$ with rhodium(I). The synthesis of $\mathbf{3 0}$ was reported by the Milstein group, but no ${ }^{31} \mathrm{P}\left\{{ }^{1} \mathrm{H}\right\}$ NMR data were obtained due 


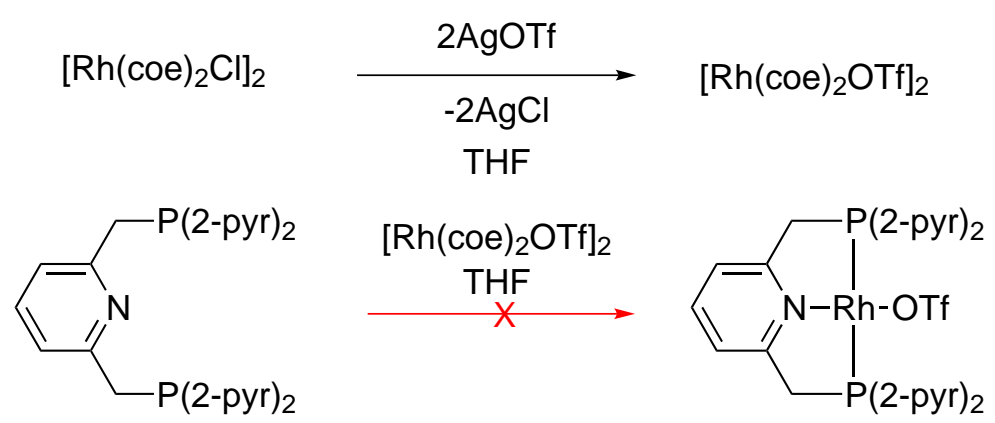

Scheme 3.5 Attempted synthesis of [(PNP)Rh(OTf)].

to its paramagnetic nature. ${ }^{126}$ In this work, ${ }^{t} \mathrm{Bu} \mathrm{PNP}$ was reacted with $\left[\mathrm{Rh}(\mathrm{coe}){ }_{2} \mathrm{Cl}\right]_{2}$ in $\mathrm{C}_{6} \mathrm{D}_{6}$ to give [( $\left.\left.{ }^{t} \mathrm{Bu} P \mathrm{PP}\right) \mathrm{RhCl}\right]$. NMR data matched the reported data $\left(\delta_{P} 52.4\right.$, ${ }^{1} J_{\mathrm{Rh}-\mathrm{P}}=143 \mathrm{~Hz}$, acetone- $\left.\mathrm{d}_{6}\right)$. This complex was then treated with one equivalent of $\mathrm{AgBF}_{4}$ (Scheme 3.6).
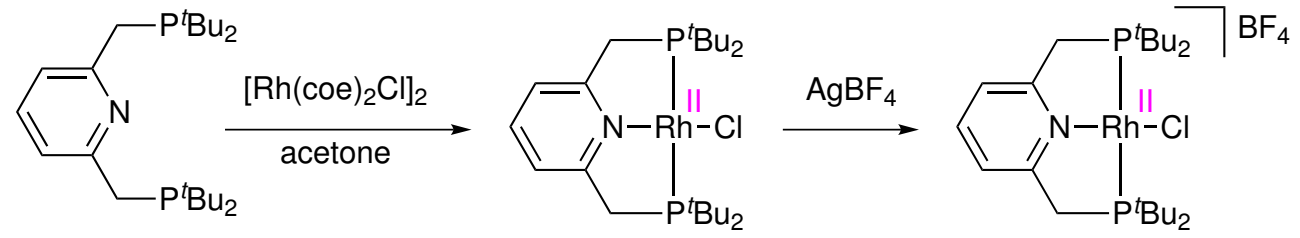

30

Scheme 3.6 Synthesis of a known rhodium(II) complex. ${ }^{126}$

Consistent with reported data, the ${ }^{1} \mathrm{H}$ NMR spectrum only contained signals from the solvent. The aromatic region was a small unresolved hump. No signals were observed in a standard ${ }^{31} \mathrm{P}\left\{{ }^{1} \mathrm{H}\right\}$ NMR spectrum $\left(\mathrm{d} 1=1 \mathrm{~s}\right.$, at=1 s). The ${ }^{31} \mathrm{P}\left\{{ }^{1} \mathrm{H}\right\}$ NMR signal of the paramagnetic rhodium(II) species appeared at $86 \mathrm{ppm}\left(\nu_{1 / 2}=590\right.$ $\mathrm{Hz}$ ) when the acquisition time was decreased to $0.05 \mathrm{~s}$. The appearance of this signal supports the conclusion that similar paramagnetic species are present in the reactions of ${ }^{2-p y r}$ PNP with rhodium(I).

Although the silver chloride byproduct resulting from the synthesis of $\left[\mathrm{Rh}(\mathrm{coe})_{2}(\mu-\right.$ $\mathrm{OTf}]_{2}$ was filtered onto a very fine frit, it is possible that any excess silver ions in the reaction mixture could oxidise rhodium(I) intermediates and form paramagnetic ${ }^{2-p y r}$ PNP complexes. To determine whether this was the case, the chloride abstraction reactions should be carried out with sodium or thallium salts. Furthermore, formation of any rhodium(II) species may be prevented by the addition of a reductant such as zinc powder. However, the appearance and chemical shift of the counterion signals in both reactions suggest that it is not just a small amount of paramagnetic impurity that has formed. 


\subsection{Concluding Remarks}

${ }^{2-p y r} \mathrm{PNP}$ is able to coordinate to rhodium. However, ${ }^{2-p y r} \mathrm{PNP}$ shows very different reactivity compared to previously reported PNP ligands. Out of all the reactions discussed in this chapter, no new rhodium complexes were isolated in good yield; furthermore, few ${ }^{2-p y r}$ PNP complexes could be identified by NMR spectroscopy. Even when reactions were carried out in toluene or acetone, solvents that are less reactive than chloroform, the typical reactivity of PNP ligands was not observed. This is particularly obvious in the electronic properties of the resulting complexes. In four of the five reactions described above, ${ }^{31} \mathrm{P}\left\{{ }^{1} \mathrm{H}\right\}$ NMR signals were only observed when the parameters of the ${ }^{31} \mathrm{P}\left\{{ }^{1} \mathrm{H}\right\}$ NMR experiment were modified. Synthesis of a known rhodium(II) complex suggested that the modified ${ }^{31} \mathrm{P}\left\{{ }^{1} \mathrm{H}\right\}$ NMR experiment is able to detect paramagnetic species and that these are present in many reactions of ${ }^{2-p y r} \mathrm{PNP}$ with rhodium. ${ }^{126}$ However, the identities of these complexes were not determined.

It is noteworthy that a silver salt was required to oxidise the rhodium(I) ${ }^{t} \mathrm{Bu} P N P$ complex but that paramagnetic ${ }^{2-p y r} \mathrm{PNP}$ complexes may have been formed in reactions where silver was not employed. This reactivity may be facilitated by the ${ }^{2-p y r} \mathrm{PNP}$ ligand. The ability of the PNP ligand platform to undergo chemically "non-innocent" reactivity has been exploited in numerous catalytic reactions. However, the redox non-innocence of these ligands is an "open question". 132

\subsection{Future Work}

The isolation of mononuclear rhodium(II) complexes is rare. Rhodium(II) complexes are usually bimetallic or require chelating ligands to protect the metal centre from further redox reactions; for this reason, it is not surprising that bulky pincers like ${ }^{t}$ Bu PNP and the diarylamido-based ligand 4-Me-2- $\left.\left({ }^{i} \operatorname{Pr}_{2} \mathrm{P}\right)-\mathrm{C}_{6} \mathrm{H}_{3}\right)_{2} \mathrm{~N}$ have been able to form stable monomeric rhodium(II) complexes. ${ }^{126,131}$ Rhodium(II) is interesting because radical chemistry has the potential to activate bonds homolytically in contrast to the typical heterolytic reactions observed with the $\mathrm{Rh}(\mathrm{I}) / \mathrm{Rh}(\mathrm{III})$ couple.

Compared to cobalt, rhodium does not usually undergo one-electron chemistry. The Chirik group has studied cobalt ${ }^{t}$ Bu PNP complexes in detail and concluded that the methylene $\mathrm{C}-\mathrm{H}$ bonds are much weaker in cobalt complexes than their rhodium analogues. This has been attributed to the tendency of cobalt to undergo $\mathrm{Co}$ (I) $/ \mathrm{Co}$ (II) oxidation much more readily than second- or third-row metals. ${ }^{132}$ Curiously, it appears 
that ${ }^{2-p y r} \mathrm{PNP}$ allows rhodium complexes to undergo one-electron chemistry quite easily. EPR and NMR spectroscopy (the Evans method in particular) should be carried out to confirm (or rule out) the presence of unpaired electrons. If unpaired electrons are present, computational chemistry could be used to determine whether the charge is located on the rhodium centre or if it is found on the ligand.

More work is required to identify the exact nature of the products of reaction of ${ }^{2-p y r}$ PNP with rhodium(I) precursors. Firstly, a solvent or solvent mixture that is less reactive than chloroform should be used for any reactions of ${ }^{2-p y r} \mathrm{PNP}$ with rhodium(I). This is particularly important if radical species are formed. The reactions should also be carried out on a larger scale so that crystallisations can be attempted - single crystals suitable for X-ray diffraction will be helpful in determining the identities of the complexes if they are indeed paramagnetic'. 


\section{Chapter 4}

\section{Iridium Complexes}

Iridium(I) diphosphine complexes have been employed in a number of catalytic reactions and provide an entry-point into iridium(III) chemistry. ${ }^{125}$ In order to catalyse a hydration reaction, the iridium complex may need to form Ir-O bonds. A number of pincer complexes containing such bonds have been reported. For example, Hartwig et al. have reported a PCP hydrido-hydroxo complex and a ${ }^{{ } \mathrm{Bu}} \mathrm{PCP}$ complex has been employed in the catalytic hydroetherification of alkenes. ${ }^{35,133}$ ColeHamilton et al. have recently reported $\alpha-\mathrm{C}-\mathrm{H}$ activation of methyl propanoate by $\left[\left({ }^{t}\right.\right.$ Bu $\left.\left.\mathrm{PNP}\right) \operatorname{Ir}(\mathrm{coe})\right] \mathrm{PF}_{6} \cdot{ }^{134}$ The resulting complex contains two Ir-O bonds. ${ }^{134}$ Their work exploited the beneficial effect of water on $\mathrm{C}-\mathrm{H}$ activation that was first reported for a PNP complex by Milstein et al. in 2006. ${ }^{135}$

Although iridium is not NMR-active, it shares properties with its lighter congener that makes it a good choice for studying pincer-catalysed hydration. For example, both iridium and rhodium are able to stabilise a wide range of coordination geometries and both have been shown to activate water. In addition to these important similarities, iridium complexes are sometimes less reactive towards chlorinated solvents than those of rhodium. This may prevent the oxidation by chloroform that was described in chapter three. ${ }^{125}$

This chapter describes the coordination chemistry of ${ }^{2-p y r} \mathrm{PNP}$ with iridium. Section 4.1 describes reactions of ${ }^{2-p y r} \mathrm{PNP}$ with cyclooctene-ligated iridium precursors. Section 4.2 discusses the reactivity of ${ }^{2-p y r} \mathrm{PNP}$ with $[\operatorname{Ir}(\operatorname{cod})(\mu-\mathrm{Cl})]_{2}$ and the structural characterisation of the resulting complex. Finally, section 4.3 explores the reactivity of cod complex [(2-pyrPNP) $\operatorname{Ir}(\mathrm{cod})] \mathrm{Cl}$ with ethene and hydrogen. 


\subsection{Reactions of ${ }^{2-p y r} \mathrm{PNP}$ with $\left[\operatorname{Ir}(\operatorname{coe})_{2}(\mu-\mathrm{Cl})\right]_{2}$ and $\left[\operatorname{Ir}(\text { acetone })_{2}(\text { coe })_{2}\right]$}

\subsection{1 $\left[\operatorname{Ir}(\operatorname{coe})_{2}(\mu-\mathrm{Cl})\right]_{2}$}

The Milstein group has reported the reaction of $\left[\operatorname{Ir}(\operatorname{coe})_{2}(\mu-\mathrm{Cl})\right]_{2}$ and $\left[\operatorname{Ir}(\operatorname{cod})_{2}\right] \mathrm{BF}_{4}$ with the bulky and electron-rich ${ }^{t}$ BuPNP ligand. ${ }^{79}$ In each case, instead of forming an iridium(I) complex, the products of vinylic $\mathrm{C}-\mathrm{H}$ activation were isolated (31 and 32, Scheme 4.1).

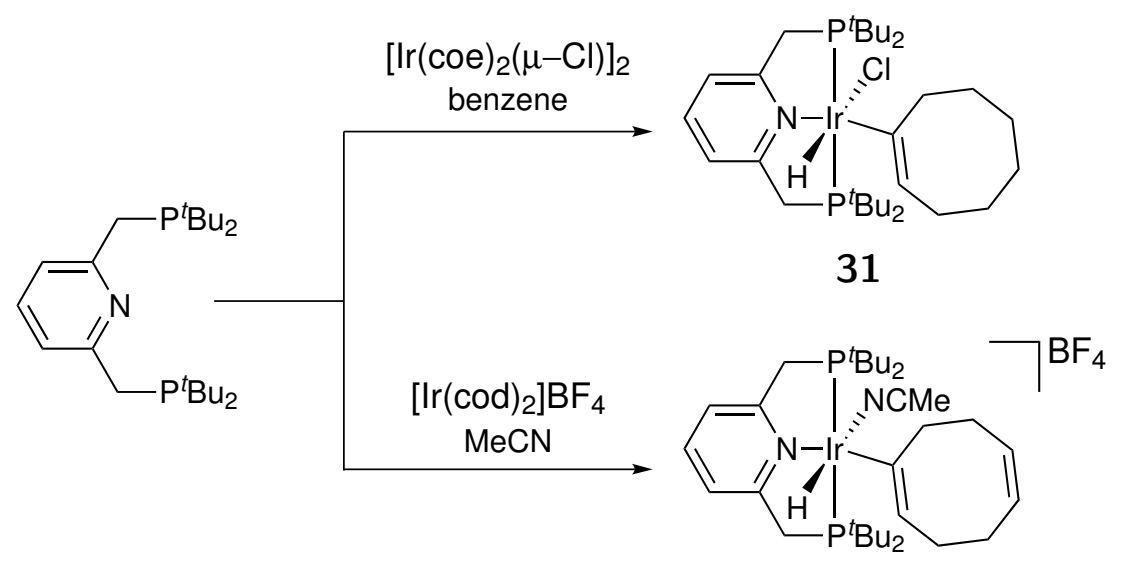

32

Scheme 4.1 Vinylic C-H activation in the reaction of ${ }^{t} \mathrm{Bu} P N P$ with $\left[\operatorname{Ir}(\mathrm{coe})_{2}(\mu-\mathrm{Cl})\right]_{2}$ and $\left[\operatorname{Ir}(\operatorname{cod})_{2}\right] \mathrm{BF}_{4} \cdot{ }^{79}$

To compare the reactivity of ${ }^{2-p y r} \mathrm{PNP}$ with that of ${ }^{t}$ BuPNP, a $2: 1$ mixture of ${ }^{2-p y r} \mathrm{PNP}$ and $\left[\operatorname{Ir}(\mathrm{coe})_{2}(\mu-\mathrm{Cl})\right]_{2}$ was suspended in toluene and allowed to stir at room temperature for 3 hours. The resulting orange solid was dissolved in $\mathrm{CD}_{2} \mathrm{Cl}_{2}$ to give a clear red solution. Three very broad signals are present in the ${ }^{31} \mathrm{P}\left\{{ }^{1} \mathrm{H}\right\}$ NMR spectrum at $\delta_{P} 26.3,19.9$, and $4.0 \mathrm{ppm}$. The ${ }^{1} \mathrm{H}$ NMR spectrum is complicated. Of note is the small signal at $\delta_{H}-18.37 \mathrm{ppm}$ and the unresolved aromatic region. This made the aromatic region difficult to interpret, except to note the absence of signals above $9 \mathrm{ppm}$ that suggests the pyridyl nitrogens are neither coordinated to a metal nor protonated. ${ }^{103}$ The chemical shift of the hydride signal suggests that the hydride ligand is located trans to $\mathrm{Cl}^{-}$on an octahedral complex. ${ }^{136}$

On formation of either $\mathbf{3 1}$ or $\mathbf{3 2}$, the ${ }^{31} \mathrm{P}\left\{{ }^{1} \mathrm{H}\right\}$ NMR signal of ${ }^{t} \mathrm{Bu} P N P$ shifted approximately $2 \mathrm{ppm}$ downfield. ${ }^{79}$ The signal for uncoordinated ${ }^{2 \text {-pyr }} \mathrm{PNP}$ is found at $\delta_{P}-6.48 \mathrm{ppm}$, but none of the observed ${ }^{31} \mathrm{P}\left\{{ }^{1} \mathrm{H}\right\} \mathrm{NMR}$ resonances are a similar distance from this signal. This suggests that ${ }^{2-p y r} \mathrm{PNP}$ has dramatically different reactivity to ${ }^{t} \mathrm{Bu} \mathrm{PNP}$; however, the large difference between the two ligands is an 
important factor to consider when comparing such values. Nevertheless, when the ${ }^{2-p y r} \mathrm{PNP}$ iridium(III) di(hydride) complex $\left[\left({ }^{2-p y r} \mathrm{PNP}\right) \operatorname{Ir}(\mathrm{H}){ }_{2} \mathrm{Cl}\right]$ was synthesised (section 4.3), the change in ${ }^{31} \mathrm{P}\left\{{ }^{1} \mathrm{H}\right\} \mathrm{NMR}$ chemical shift from the free ligand to the complex was comparable to the value obtained for the analogous ${ }^{t} \mathrm{Bu} \mathrm{PNP}$ complex. ${ }^{137}$

A ${ }^{1} \mathrm{H}-{ }^{31} \mathrm{P}$ HMBC experiment was carried out in order to obtain clues about the identities of the complexes. A correlation between the ${ }^{31} \mathrm{P}$ signal at $\delta_{P} 19.9 \mathrm{ppm}$ and two resonances at $\delta_{H} 5.26$ and $4.55 \mathrm{ppm}$ suggests that the ${ }^{2-p y r} \mathrm{PNP}$ complex responsible contains diastereotopic $\mathrm{P}-\mathrm{CH}_{2}$ protons. This would mean it lacks a mirror plane bisecting the ligand backbone. Apart from this information, no further information could be gleaned from this spectrum. Neither elemental analysis nor an infrared spectrum were obtained as the reaction does not produce a single product.

Purification of the reaction mixture by fractional crystallisation was attempted by slow diffusion of diethyl ether into a concentrated dichloromethane solution of the mixture. The bulk of the isolated material was insoluble in deuterated solvents. A very weak NMR spectrum of a ${ }^{2-p y r} \mathrm{PNP}$-containing species was obtained in $\mathrm{CDCl}_{3}$, but it remained impure and represented a small fraction of the total amount of material. Repeating the reaction and crystallisation on a much larger scale may help in identifying the components of the mixture. However, this may not be worthwhile as the final yield will be very small.

${ }^{2-p y r} \mathrm{PNP}$ was reacted with $\left[\operatorname{Ir}(\mathrm{coe})_{2}(\mu \text {-Cl })\right]_{2}$ and a halide acceptor in the hope that by removing the chloride anion, a single complex would form. Silver salts are often employed for this type of reaction but were avoided in this case because of the potential for silver to coordinate to the pyridyl nitrogen atoms or act as an oxidant. ${ }^{138}$ 2-pyr PNP was combined with $\left[\operatorname{Ir}(\text { coe })_{2}(\mu-\mathrm{Cl})\right]_{2}$ and $\mathrm{NaBPh}_{4}$ in $\mathrm{CDCl}_{3}$. The ${ }^{31} \mathrm{P}\left\{{ }^{1} \mathrm{H}\right\}$ NMR spectrum displays a very broad unresolved signal at $4.1 \mathrm{ppm}$, a singlet at $19.6 \mathrm{ppm}$, and a signal for ${ }^{2-\mathrm{pyr}} \mathrm{PNP}_{-} \mathrm{O}_{2}$ at $26.3 \mathrm{ppm}$. The chemical shifts of these signals are very similar to those present when the reaction is carried out in the absence of $\mathrm{NaBPh}_{4}$; however, after this reaction, the signal at $\delta_{P} 4.13 \mathrm{ppm}$ is much more intense. The ${ }^{1} \mathrm{H}$ NMR spectrum is also very complicated. The absence of aromatic signals above 9 ppm suggests that none of the pyridyl nitrogens are coordinated to a metal. ${ }^{103}$

NMR data alone were not sufficient to determine the identity of any of the complexes formed in this reaction. 


\subsection{2 $\left[\operatorname{Ir}(\text { acetone })_{2}(\text { coe })_{2}\right] \mathrm{PF}_{6}$}

The most well-studied iridium(I) PNP pincer complex is $\left[{ }^{t} \mathrm{Bu} P N P\right) \operatorname{Ir}($ coe $\left.)\right] \mathrm{X}\left(\mathrm{X}=\mathrm{PF}_{6}\right.$, $\left.\mathrm{BArF}, \mathrm{BF}_{4}\right)$. This is synthesised by reacting ${ }^{t} \mathrm{Bu} \mathrm{PNP}$ with $\left.[\operatorname{Ir} \text { (acetone })_{2}(\text { coe })_{2}\right] \mathrm{X}$ in acetone. ${ }^{139}$ Because this is the direct precursor to many complexes, including the neutral dearomatised complex $\left.\left[{ }^{t}{ }^{B}{ }^{P N P} *\right) \operatorname{Ir}(\mathrm{coe})\right]$, it was important to determine whether the ${ }^{2-\text { pyr }}$ PNP analogue could be synthesised. ${ }^{135}$

The iridium(I) precursor $\left.[\operatorname{Ir} \text { (acetone })_{2}(\text { coe })_{2}\right] \mathrm{PF}_{6}$ was synthesised by reacting $\left[\operatorname{Ir}(\text { coe })_{2}\right.$ $(\mu-\mathrm{Cl})]_{2}$ with $\mathrm{AgPF}_{6}$ in acetone, then removing the $\mathrm{AgCl}$ byproduct by filtration. The acetone solution was then added to ${ }^{2-p y r} \mathrm{PNP}$ (Scheme 4.2).
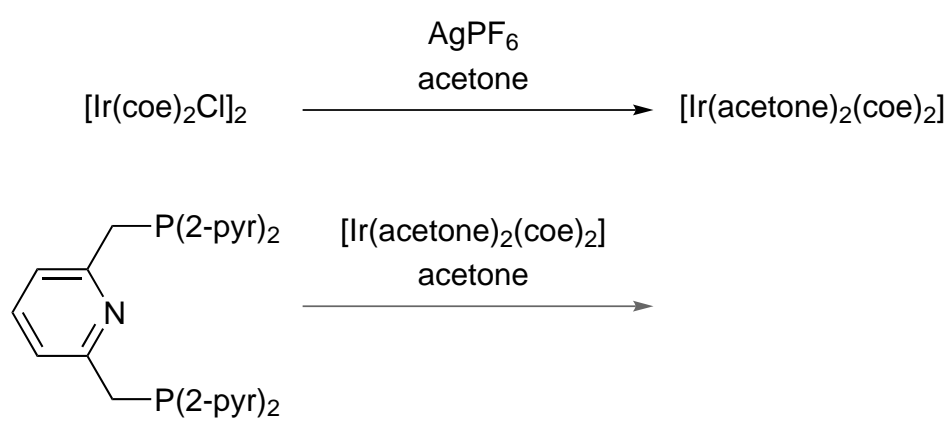

Scheme 4.2 Reaction of $\left[\operatorname{Ir}(\text { acetone })_{2}(\text { coe })_{2}\right] \mathrm{PF}_{6}$ with ${ }^{2-\mathrm{pyr}} \mathrm{PNP}$.

The ${ }^{1} \mathrm{H}$ NMR spectrum bore some resemblance to the spectra obtained when ${ }^{2-p y r} \mathrm{PNP}$ was reacted with $\left[\operatorname{Ir}(\mathrm{coe})_{2}(\mu-\mathrm{Cl})\right]_{2}$ : no signals for coordinated cyclooctene could be found, the aromatic region was complicated, and there was evidence for $\mathrm{Ir}-\mathrm{H}$ bonds in the form of two signals in a 1:1 ratio $\left(\delta_{H}-17.4\right.$ and $\left.-18.3 \mathrm{ppm}\right)$. Again, the chemical shift of the hydrides suggests they are trans to a chlorido ligand. ${ }^{136}$ The ${ }^{31} \mathrm{P}\left\{{ }^{1} \mathrm{H}\right\}$ NMR spectrum contained four closely-spaced resonances near $\delta_{P} 20 \mathrm{ppm}$ and two unresolved sets of signals at $\delta_{P} 12.4$ and $3.3 \mathrm{ppm}$. The septet signal of $\mathrm{PF}_{6}{ }^{-}$was sharp and appeared at $\delta_{P}-144.0 \mathrm{ppm}$.

In summary, when ${ }^{2-p y r} \mathrm{PNP}$ is reacted with cyclooctene-ligated iridium(I) precursors, mixtures of ${ }^{2-p y r} \mathrm{PNP}$ complexes form that are very difficult to separate and characterise.

\subsection{Reactions of ${ }^{2-p y r} \mathrm{PNP}$ with $[\operatorname{Ir}(\operatorname{cod})(\mu-\mathrm{Cl})]_{2}$ and $[\operatorname{Ir}(\operatorname{cod})(\mu-\mathrm{OMe})]_{2}$}

Reactions of appropriate phosphines with iridium(I) dimers bearing cod ligands have resulted in effective pre-catalysts suitable for a number of reactions. These include 
hydrogenation, hydroetherification, and allylic substitution. ${ }^{125,133,140}$ Such complexes are synthesised by reacting the ligand with $[\operatorname{Ir}(\operatorname{cod})(\mu-\mathrm{Cl})]_{2}$ or $[\operatorname{Ir}(\operatorname{cod})(\mu-\mathrm{OMe})]_{2}$ in the presence of a halide acceptor or with the iridium(I) precursor alone. The reactivity of ${ }^{2-p y r} \mathrm{PNP}$ with coe complexes has been discussed in chapter three and in section 4.1 above, and has typically been complicated. While $\eta^{2}$-coe is usually easier to displace from $\left[\operatorname{Ir}(\operatorname{coe})_{2}(\mu-\mathrm{Cl})\right]_{2}$ than chelated $\eta^{4}$-cod from $[\operatorname{Ir}(\operatorname{cod})(\mu-\mathrm{Cl})]_{2}$, cod can form chelating products that may lead to more stable ${ }^{2-p y r} \mathrm{PNP}$ complexes.

Different products have been reported depending on the ligand. For example, when the neopentyl-substituted ligand ${ }^{t} \mathrm{BuCH}_{2} \mathrm{PNP}$ is reacted with $[\operatorname{Ir}(\operatorname{cod})(\mu-\mathrm{Cl})]_{2}$, the chelating cod ligand is displaced and one of the ${ }^{t} \mathrm{Bu}$ groups is metallated (Figure 4.1A). ${ }^{76}$ In the case of ${ }^{\mathrm{Ph} P N P}$, the cationic $\eta^{4}$-cod complex is formed (Figure 4.1-B). This complex is able to hydrogenate imines. ${ }^{112}$ The only reported reaction of ${ }^{t} \mathrm{Bu} P N P$ with an iridium(I) precursor bearing cod ligands gave compound 32 (Scheme 4.1). There are no reports of neutral PNP ligands having been reacted with $[\operatorname{Ir}(\operatorname{cod})(\mu-\mathrm{OMe})]_{2}$. This precursor has been used to synthesise numerous hydrogenation catalysts, as well as a triphosphine PPP complex where the phosphido backbone is generated through deprotonation by methoxide (Figure 4.1-C). ${ }^{125,141}$

A
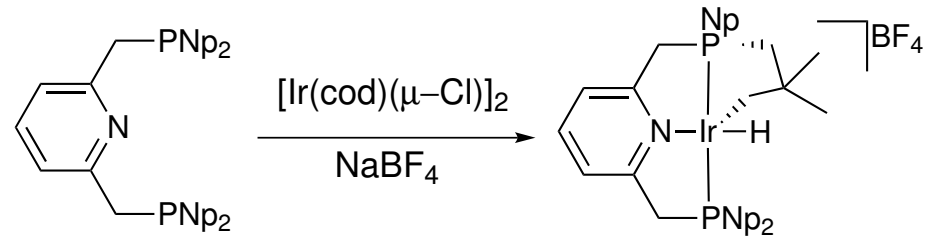

B
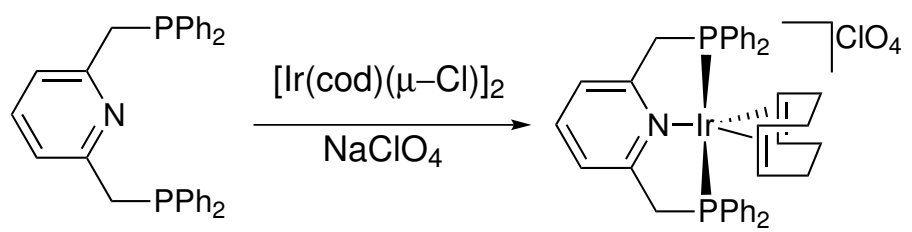

C

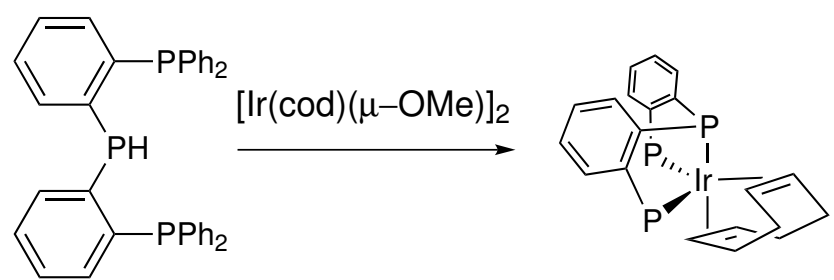

Figure 4.1 Examples of the reactivity of PNP and PPP ligands with iridium(I) complexes bearing $\eta^{4}$-1,5-cyclooctadiene (cod). ${ }^{112,141,142}$

This section describes how ${ }^{2-p y r} \mathrm{PNP}$ compares to reported PNP pincer ligands in reactions with iridium(I) precursors bearing cod ligands. 


\subsection{1 $[\operatorname{Ir}(\operatorname{cod})(\mu-\mathrm{Cl})]_{2}$}

Whereas ${ }^{2-p y r} \mathrm{PNP}$ showed complicated reactivity with $[\mathrm{Rh}(\operatorname{cod})(\mu-\mathrm{Cl})]_{2}$, its reaction with $[\operatorname{Ir}(\operatorname{cod})(\mu-\mathrm{Cl})]_{2}$ in $\mathrm{CDCl}_{3}$ at room temperature formed a single product $(\mathbf{3 3})$.

The ${ }^{1} \mathrm{H}$ NMR spectrum of $\mathbf{3 3}$ in $\mathrm{CDCl}_{3}$ displayed eight signals for the pyridyl protons. A COSY spectrum revealed that these signals were part of two separate spin systems. Six signals were observed for the cyclooctadiene protons, and no free cyclooctadiene was present; furthermore, rather than appearing as a doublet or virtual triplet, the $\mathrm{P}-\mathrm{CH}_{2}$ groups appeared as an $\mathrm{AB}$ system. The presence of diastereotopic protons on the ligand backbone, combined with the two sets of pyridyl protons, suggests that the complex has $\mathrm{C}_{\mathrm{s}}$ symmetry. The ${ }^{31} \mathrm{P}\left\{{ }^{1} \mathrm{H}\right\}$ NMR spectrum showed a singlet at $\delta_{P} 13.8$ ppm, confirming that the mirror plane required by $\mathrm{C}_{\mathrm{s}}$ symmetry is perpendicular to the backbone of the pyridine ring. This makes the two phosphorus atoms equivalent but allows the pyridyl rings to differ.

Nitrogen-15 NMR data were collected to confirm that the backbone nitrogen is coordinated to iridium. These data are more difficult to obtain than those of other common nuclei. ${ }^{14} \mathrm{~N}$ is a quadrupolar nucleus so has very broad NMR resonances, and although ${ }^{15} \mathrm{~N}$ is spin $1 / 2$, it is only $0.37 \%$ abundant and has a very low gyromagnetic ratio. Heteronuclear correlation experiments are usually used to measure ${ }^{15} \mathrm{~N}$ chemical shifts, as they offer an improvement in sensitivity over direct detection. In this work, ${ }^{15} \mathrm{~N}$ data were obtained using a ${ }^{1} \mathrm{H}-{ }^{15} \mathrm{~N}$ CIGAR-HMBC experiment. In a standard ${ }^{1} \mathrm{H}-{ }^{13} \mathrm{C}$ HMBC experiment, the range of ${ }^{1} \mathrm{H}-{ }^{13} \mathrm{C}$ coupling constants is quite predictable and a fixed value can be used. This is not the case for ${ }^{1} \mathrm{H}-{ }^{15} \mathrm{~N}$ coupling constants, which can vary significantly. ${ }^{143}$ The ${ }^{1} \mathrm{H}-{ }^{15} \mathrm{~N}$ CIGAR-HMBC experiment has the ability to sample a range of ${ }^{1} \mathrm{H}-{ }^{15} \mathrm{~N}$ coupling constants, making it an improvement over the HMBC pulse sequence, and making identification of all the ${ }^{15} \mathrm{~N}$ resonances in the sample more reliable.

An expansion of the ${ }^{1} \mathrm{H}-{ }^{15} \mathrm{~N}$ CIGAR-HMBC spectrum of 33 is shown in Figure 4.2. This particular region revealed the ${ }^{15} \mathrm{~N}$ chemical shifts for the two sets of pyridyl nitrogen atoms. Tridentate coordination of ${ }^{2-p y r} \mathrm{PNP}$ was confirmed by the change in ${ }^{15} \mathrm{~N}$ chemical shift of the backbone nitrogen from $\delta_{N}-66.1 \mathrm{ppm}$ for uncoordinated ${ }^{2-p y r} \mathrm{PNP}$ to $-153 \mathrm{ppm}$ in $\mathbf{3 3}$ * $^{*}$

To summarise, multinuclear NMR data suggest that $\mathbf{3 3}$ is a $\mathrm{C}_{\mathrm{s}}$-symmetric 18 electron pincer complex with the formula $\left[\left({ }^{2-p y r} \mathrm{PNP}\right) \operatorname{Ir}(\operatorname{cod})\right] \mathrm{Cl}$. This formula, along with the identity of the cation, was confirmed by high resolution mass spectrometry.

${ }^{*}$ Nitrogen-15 NMR shifts referenced to nitromethane. 


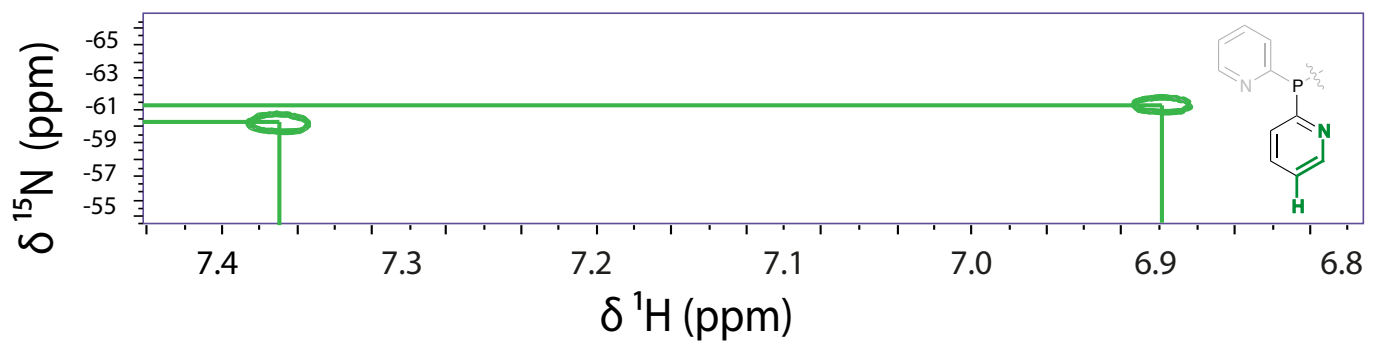

Figure 4.2 Expansion of the ${ }^{1} \mathrm{H}-{ }^{15} \mathrm{~N}$ CIGAR-HMBC spectrum showing the two pyridyl nitrogens coupling to the pyridyl protons three bonds away.

Two iridium(I) PNP cod complexes have been reported. The first, with ${ }^{\mathrm{Ph}} \mathrm{PNP}$ in a "quasi-facial coordination [mode]", was published in 1996 without either NMR data or the cited X-ray structure. ${ }^{112}$ This structure has not been deposited with the Cambridge Structural Database. The second, $\left.\left[{ }^{i} \operatorname{Pr} \mathrm{PNP}\right) \operatorname{Ir}(\operatorname{cod})\right] \mathrm{BF}_{4}$, has NMR data that suggests diastereotopic methylene protons and, therefore, a similar coordination mode to both the PhPNP complex and 33. However, the geometry of the complex is not discussed outright. ${ }^{142}$

A search of the Cambridge Structural Database (CSD) identified the X-ray structure of a single pincer cod complex, $\left[\left({ }^{\mathrm{CF}_{3} \mathrm{PCP}}\right) \operatorname{Ir}(\operatorname{cod})\right](\mathbf{3 4}) .{ }^{144}$ This has a $\mathrm{P}(1)-\mathrm{Ir}-\mathrm{P}(2)$ angle of $119.02(4)^{\circ}$, which is dramatically different from the average P-Ir-P angle of all five-coordinate iridium PCP and PNP complexes $\left(159.2^{\circ}\right){ }^{*}$ The distorted trigonal bipyramidal geometry of this complex is shown in Figure 4.3. $\mathrm{CF}_{3} \mathrm{PCP}$ has strongly $\pi$-accepting phosphorus donor atoms, which give it unique properties through its ability to strongly influence the electronic and geometric preferences of the metal.
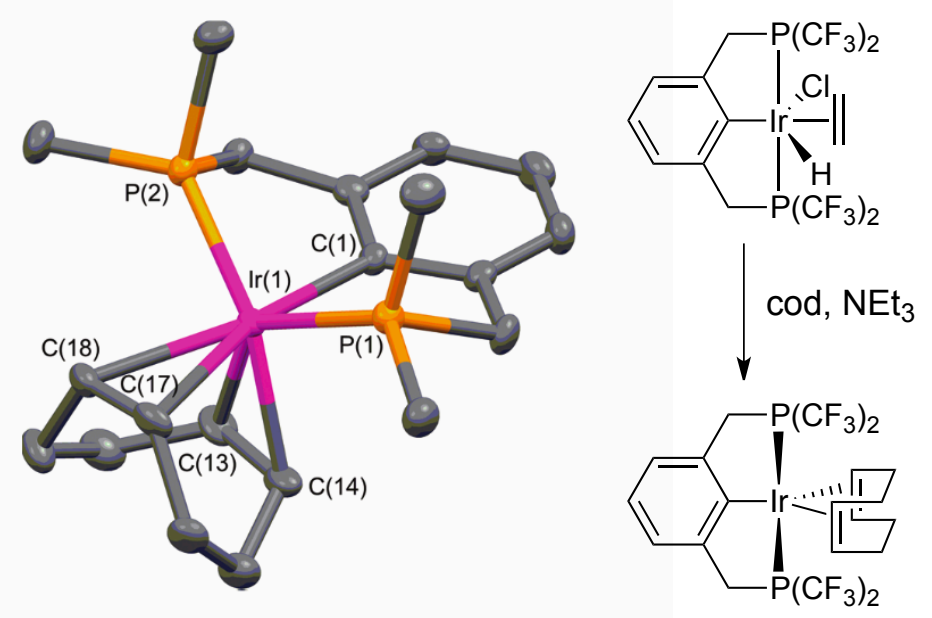

Figure 4.3 X-ray structure and synthesis of $\left[\left({ }^{\mathrm{CF}_{3} \mathrm{PCP}}\right) \operatorname{Ir}(\operatorname{cod})\right]$, 34. Structure reproduced from Adams, Organometallics, 2011, with hydrogen atoms removed for clarity. ${ }^{144}$

${ }^{*}$ CSD search January 2015. Average angle across 42 complexes 
Although $\mathrm{CF}_{3} \mathrm{PCP}$ is very different to ${ }^{2-\mathrm{pyr}} \mathrm{PNP}$, particularly with respect to its electronic properties, NMR data suggested that $\mathbf{3 3}$ and $\mathbf{3 4}$ have similar geometries. Akin to having two sets of pyridyl signals, distinct ${ }^{19} \mathrm{~F}$ NMR signals for the two sets of $\mathrm{CF}_{3}$ groups were reported. Also noteworthy is the similarity in chemical shift of the cod alkene protons. This is further evidence for a facially coordinated ligand. In the proposed structure, the cyclooctadiene ligand spans axial and equatorial positions, and such a coordination mode will result in distinctive chemical shifts for the alkene protons.

Single crystals of 33 were grown by slow diffusion of diethyl ether into a $\mathrm{CDCl}_{3}$ solution at $-35{ }^{\circ} \mathrm{C}$. This allowed the determination of the X-ray structure shown in Figure 4.4. The structure confirms the connectivity determined by NMR spectroscopy and provides unambiguous evidence for facially coordinated ${ }^{2-p y r} \mathrm{PNP}$. This non-meridional geometry is rare for a pincer ligand and is very similar to that of $\left[\left({ }^{\mathrm{CF}_{3} \mathrm{PCP}}\right) \operatorname{Ir}(\mathrm{cod})\right] \cdot{ }^{144}$ It is also likely to be the same as the analogous ${ }^{\mathrm{Ph}} \mathrm{PNP}$ and ${ }^{i} \mathrm{Pr} P N P$ complexes. This is the first example of a crystallographically characterised facially-coordinated PNP ligand and only the second crystallographically characterised pincer cod complex.

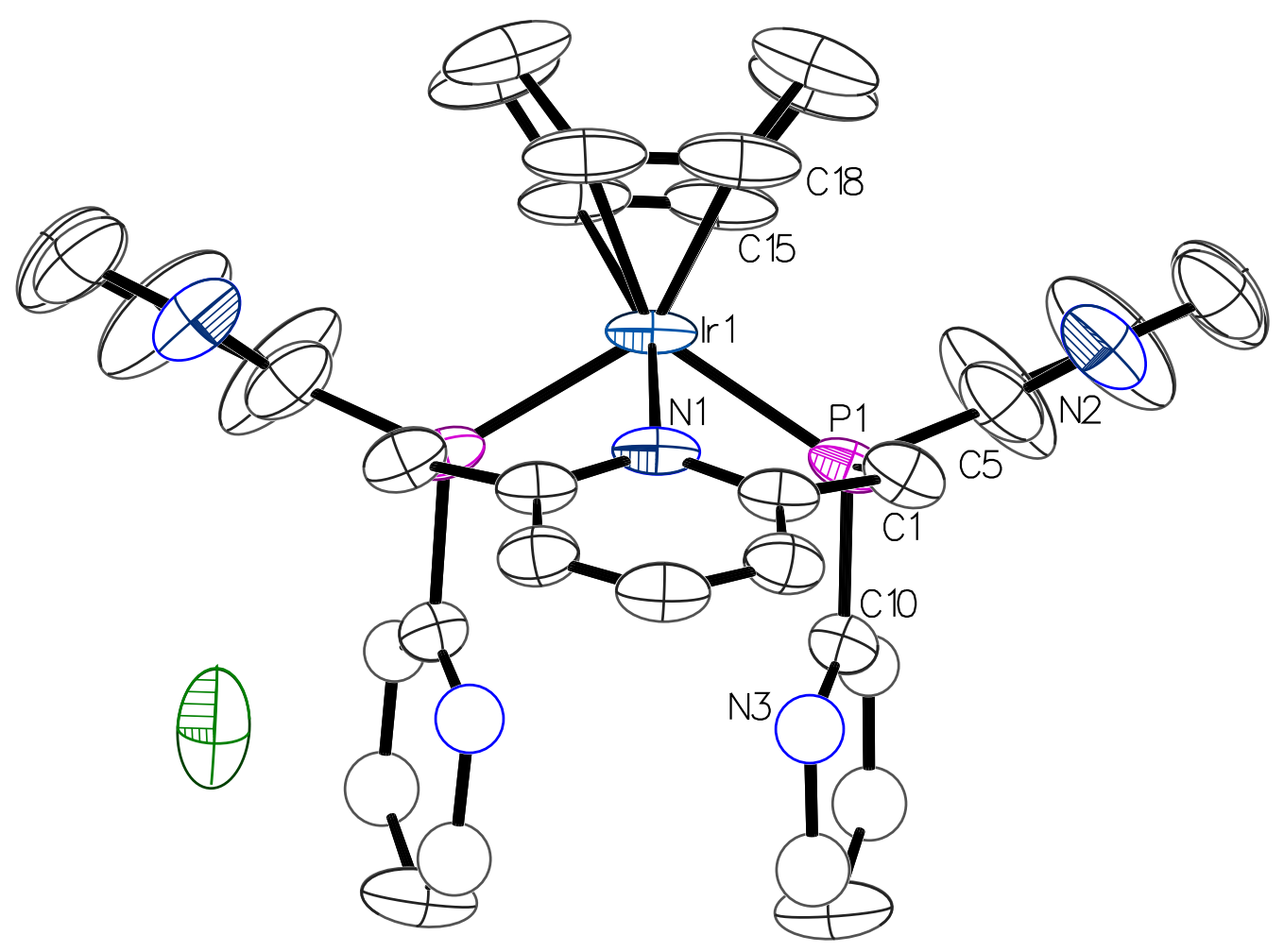

Figure 4.4 X-ray structure of $\left[\left({ }^{2-p y r} \mathrm{PNP}\right) \operatorname{Ir}(\operatorname{cod})\right] \mathrm{Cl}$.

Compound 33 crystallises in the $\mathrm{C} 2 / m$ space group and lies on a mirror plane. One set of pyridyl rings is disordered across two positions by rotation through the 
$\mathrm{P}-\mathrm{C}_{\text {pyridyl }}$ bond and was modelled isotropically.* These rings point directly away from the coordinated cod. Disordered solvent is also omitted from Figure 4.4. ${ }^{\dagger}$

Figure 4.5 shows the trigonal bipyramidal core of the complex. The average $\mathrm{P}-\mathrm{Ir} 1-\mathrm{P}$ angle for five-coordinate iridium PNP complexes in $162.69^{\circ}$. The corresponding value for 33 is $114.42(15)^{\circ}$. This is the smallest such angle for an iridium pincer complex.

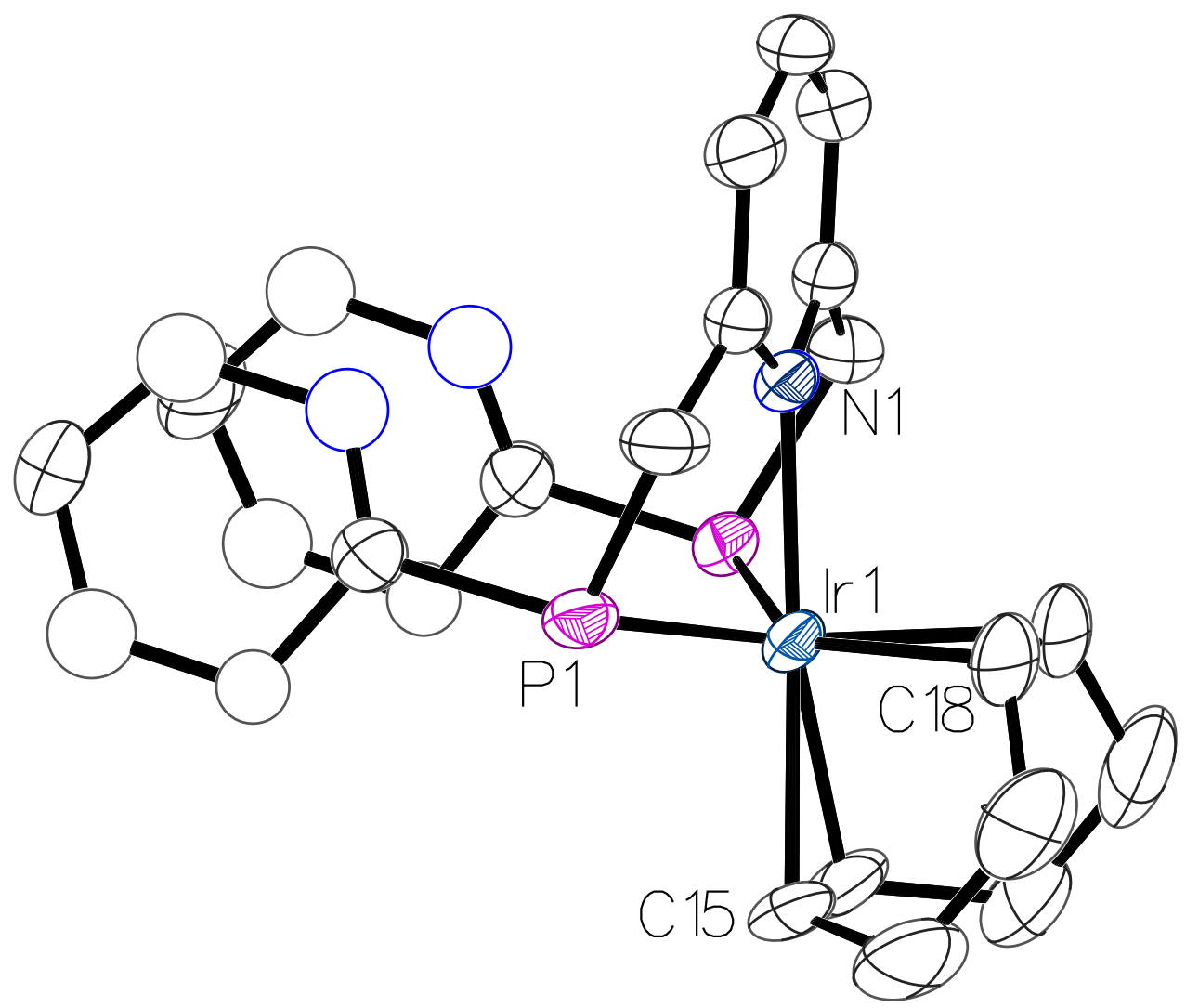

Figure 4.5 Trigonal bipyramidal core of 33; counterion, downwards-facing pyridyl groups, and disordered solvent not shown.

Compound $\mathbf{3 3}$ is insoluble in hydrocarbon solvents, diethyl ether, THF, and acetone. It is soluble in dichloromethane, chloroform, 1,4-dioxane, and methanol. An NMR spectrum of a dilute solution of $\mathbf{3 3}$ in neutral $\mathrm{D}_{2} \mathrm{O}$ was weak but acceptable. The solubility of $\mathbf{3 3}$ in water is greatly increased if a small amount of acetone is added. Wormald et al. have determined that the water-water hydrogen bond is weaker than the acetone-water hydrogen bond. ${ }^{145}$ This new network is able to solubilise 33 . When water is added to acetone, the acetone carbonyl oxygen atom is able to form hydrogen bonds to one of the hydrogen atoms of a nearby water molecule, and the methyl protons are able to interact with the oxygen atom of this water molecule. ${ }^{145}$

${ }^{*}$ Figure 4.4 shows one structure. A view of the other part can be found in Appendix A (Figure 8.2).

†Thanks to Dr Matt Polson from the University of Canterbury's X-ray Crystallography Laboratory for help with the disordered structure. 
Attempts at obtaining accurate elemental analysis data for ${ }^{2-p y r} \mathrm{PNP}$ complexes, including 33, were hampered by their hygroscopic nature. Compound 33 is particularly difficult to dry as its solubility after drying under reduced pressure changes significantly. This suggests it is unstable. Thus, $\mathbf{3 3}$ was usually synthesised and reacted immediately or stored under argon for short periods of time before use.

Preliminary investigations into anion metathesis reactions were carried out with 33 . It is often useful to synthesise cationic (pre-)catalysts with a series of counterions, particularly those that are weakly coordinating. ${ }^{125}$ Chloride counterions are also good ligands, and during a catalytic reaction it is possible that they could bind to the metal, either slowing or inhibiting the reaction. Compound 33 was reacted with one equivalent of $\mathrm{NaBPh}_{4}$ and $\mathrm{NaBArF}$, respectively. The products of these reactions could not be purified by crystallisation, which is surprising as these salts are typically employed in order to crystallise cationic complexes. Each reaction also formed an oily brown residue that was insoluble in chloroform and water. Successful anion exchange was confirmed by comparing the resulting ${ }^{1} \mathrm{H}$ NMR spectrum to that of 33 . In each case, the cod proton resonances remained in the same chemical shift, the methylene resonances were slightly shielded, and the pattern of the pyridyl signals changed. Although integration of the ${ }^{1} \mathrm{H}$ NMR spectrum of $\left[\left({ }^{2-p y r} \mathrm{PNP}\right) \operatorname{Ir}(\operatorname{cod})\right] \mathrm{NaBPh}_{4}$ was consistent with a 1:1 ratio of anion to cation, difficulties were encountered in achieving this for the NaBArF reaction. Even after carrying out a liquid-liquid separation, excess $\mathrm{BArF}^{-}$was present. Further work is required in order to optimise this reaction.

\subsubsection{Comparison of ${ }^{2-p y r} \mathrm{PNP}$ with ${ }^{\mathrm{Ph}} \mathrm{PNP}$}

${ }^{\mathrm{Ph}} \mathrm{PNP}$ was also reacted with $[\operatorname{Ir}(\operatorname{cod})(\mu-\mathrm{Cl})]_{2}$. This formed $\left[\left({ }^{\mathrm{Ph} P N P}\right) \operatorname{Ir}(\operatorname{cod})\right] \mathrm{Cl}$ (35) as a pale yellow-green solid (Scheme 4.3). The NMR data of 35 are very similar to those of 33. Synthesis of $\left[\left({ }^{\mathrm{Ph} P N P}\right) \operatorname{Ir}(\operatorname{cod})\right] \mathrm{ClO}_{4}$ has been reported but only the ${ }^{31} \mathrm{P}\left\{{ }^{1} \mathrm{H}\right\}$ NMR chemical shift was provided (singlet, $\delta_{P} 11.0 \mathrm{ppm}$ ). ${ }^{112}$ This shift is consistent with the data recorded for $\mathbf{3 5}$ - a singlet at $\delta_{P} 11.0 \mathrm{ppm}$. The diastereotopic $\mathrm{P}-\mathrm{CH}_{2}$ protons, distinctive resonances for the cod protons, and overall similarity between the NMR data of $\mathbf{3 3}$ and of $\mathbf{3 5}$ confirm that $\mathbf{3 5}$ is pyridyl-free but otherwise identical in connectivity to 33 .

The stability of 35 is similar to that of its ${ }^{2-p y r} \mathrm{PNP}$ analogue, although 35 seems to be more robust under reduced pressure. The phenyl groups enhanced its solubility in hydrocarbons and ethers compared to 33 . Attempts to crystallise $\mathbf{3 5}$ by slow diffusion of ether into dichloromethane failed as $\mathbf{3 5}$ decomposed and formed a dark brown solid that smelt strongly of cod. 


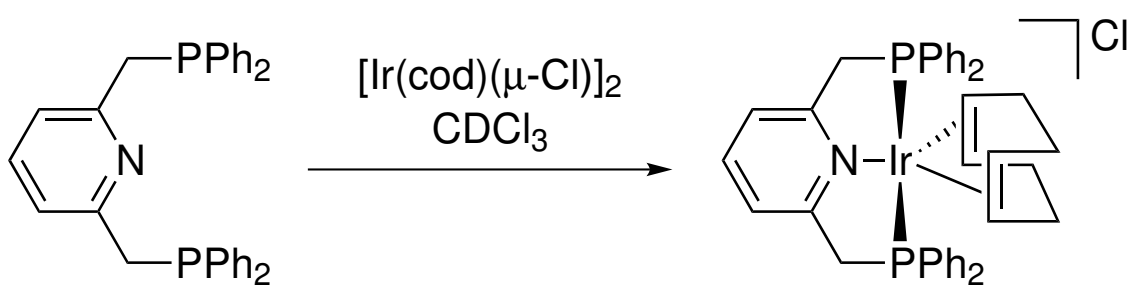

Scheme 4.3 Synthesis of 35 .

\subsection{3 $[\operatorname{Ir}(\operatorname{cod})(\mu-\mathrm{OMe})]_{2}$}

With one significant exception, the NMR data from the reaction of ${ }^{2-p y r} \mathrm{PNP}$ with $[\operatorname{Ir}(\operatorname{cod})(\mu-\mathrm{OMe})]_{2}$ in $\mathrm{CDCl}_{3}$ are very similar to those of 33 . The greatest difference between any of the ${ }^{13} \mathrm{C}$ resonances is $0.24 \mathrm{ppm}$. This slight deshielding occurs for the ispo carbons of the pyridyl rings.

Where the data differ is in the appearance of the $\mathrm{P}-\mathrm{CH}_{2}$ group: the signal for the $\mathrm{P}-\mathrm{CH}_{2}$ protons of the ligand is absent, and the appearance of the $\mathrm{P}-\mathrm{CH}_{2}$ ${ }^{13} \mathrm{C}$ resonance has changed significantly. This is due to deuterium incorporation at this position. The strongly basic nature of the methoxide anion, combined with the similarity in pKa between methanol and chloroform, means that at least one base and one source of deuterium is present in the reaction mixture. The methylene "arms" of pincer ligands are well known to be acidic ${ }^{57}$ and have been deprotonated by a number of strong bases such as potassium tert-butoxide and potassium bis(trimethylsilyl)amide. These reactions are carried out in aprotic solvents so that the neutral dearomatised complex can be isolated. ${ }^{71}$

A proposed mechanism for deuterium incorporation is shown in Figure 4.6. Deprotonation of a methylene group by methoxide results in a neutral complex that can be stabilised by resonance into the pyridine ring. The anionic backbone can then be deuterated by slightly acidic $\mathrm{CDCl}_{3}$ (pKa 15.5). ${ }^{146 *}$ Generation of the trichloromethyl anion in this reaction can result in the formation of methanol as shown in Figure 4.6. A signal at $\delta_{C} 55.05 \mathrm{ppm}$ is good evidence for this. The final product may have a chloride counterion rather than methoxide. When 33 was reacted with $\mathrm{NaBPh}_{4}$, the different counterion resulted in a much greater change to the ${ }^{1} \mathrm{H}$ NMR spectrum compared to that observed when $[\operatorname{Ir}(\operatorname{cod})(\mu-\mathrm{OMe})]_{2}$ was used as the iridium source. The chloride counterion could be generated as a result of trichloromethyl anion decomposition - reaction of sodium methoxide with chloroform is used to generate dichlorocarbene and $\mathrm{Cl}^{-}$via the trichloromethyl anion.

\footnotetext{
* Reported pKa values for chloroform vary from 15 to 24 depending on the method of determination 147
} 
Acid-base equilibrium

$\mathrm{OMe}^{-}+\mathrm{DCCl}_{3} \rightleftharpoons \mathrm{DOMe}+\mathrm{CCl}_{3}{ }^{-}$

Deuteration
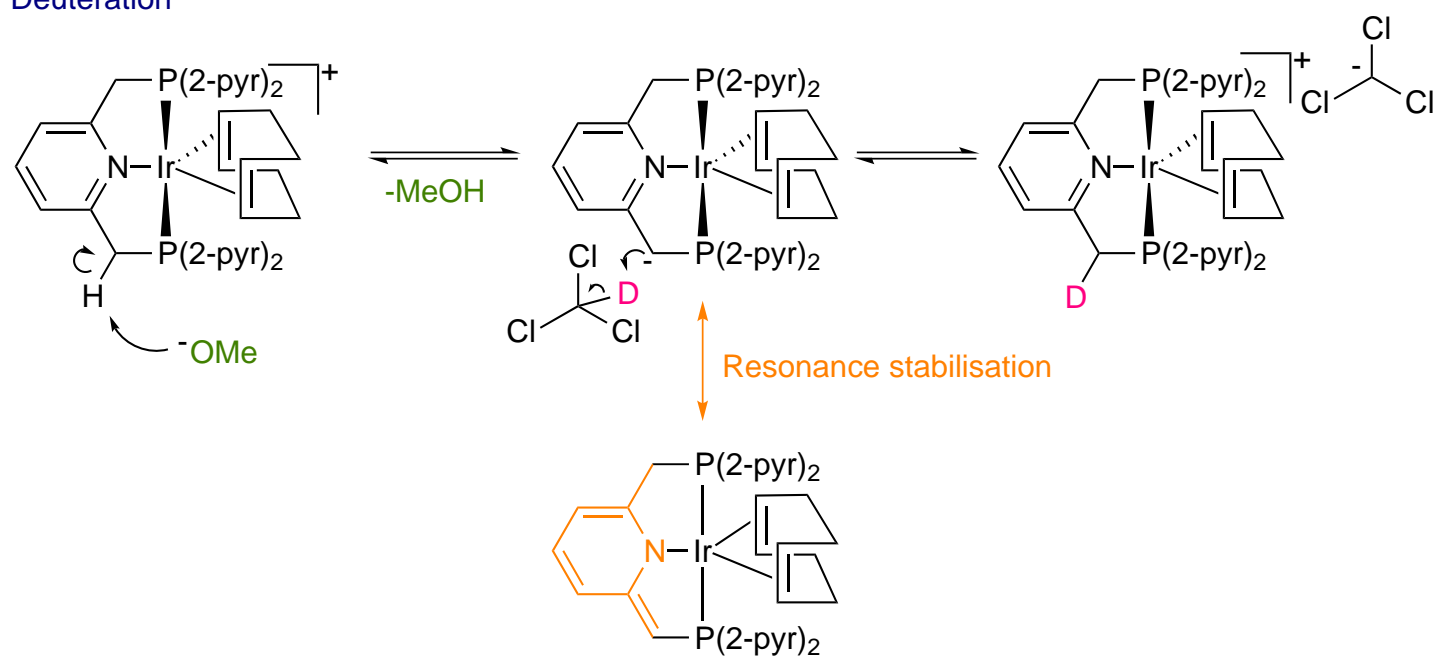

Regeneration of methoxide
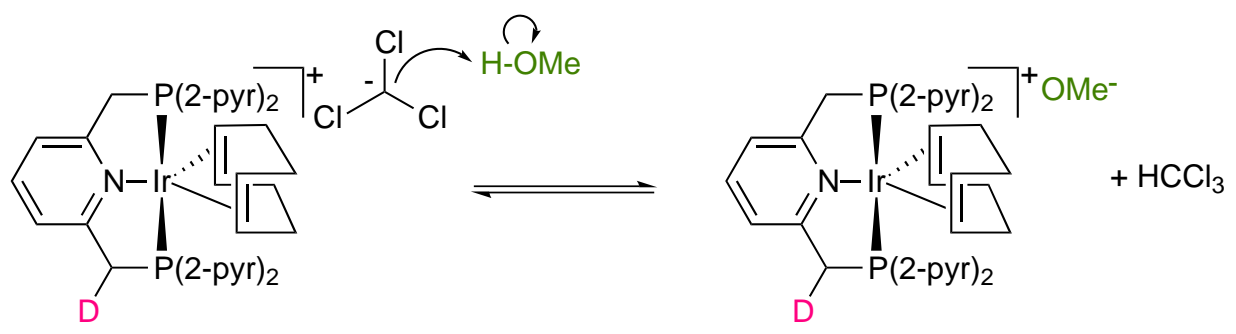

Figure 4.6 Proposed mechanism for deuterium incorporation. Dearomatised backbone of $\left[\left({ }^{2-p y r} \mathrm{PNP}^{*}\right) \operatorname{Ir}(\operatorname{cod})\right]$ shown in orange. 
The implications of this reaction are twofold. Firstly, the deprotonated complex is highly reactive, which may be beneficial if an aromatisation/dearomatisation cycle is required in a catalytic reaction. Secondly, observing the dearomatised complex $\left[\left({ }^{2-p y r} \mathrm{PNP}^{*}\right) \operatorname{Ir}(\operatorname{cod})\right]$ by NMR will be very difficult as complex 33 is not soluble in any aprotic deuterated solvents; furthermore, $\mathrm{CDCl}_{3}$ is able to provide deuterium atoms to ${ }^{2-p y r} \mathrm{PNP}$ complexes. Further investigation is needed to determine whether dichloromethane is a suitable solvent.

In the ${ }^{13} \mathrm{C}\left\{{ }^{1} \mathrm{H}\right\}$ NMR spectrum of $\mathbf{3 3}$, the $\mathrm{P}-\mathrm{CH}_{2}$ resonance is a sharp doublet at $\delta_{C}$ $44.6 \mathrm{ppm}\left({ }^{1} J_{\mathrm{P}-\mathrm{C}}=38.6 \mathrm{~Hz}\right)$ but is broad and unresolved $\left(\nu_{1 / 2}=90 \mathrm{~Hz}\right)$ when $[\operatorname{Ir}(\operatorname{cod})(\mu-$ $\mathrm{OMe})]_{2}$ is used as the iridium(I) precursor (Figure 4.7). The broadness of the signal is unsurprising as the carbon nucleus is coupled to both ${ }^{31} \mathrm{P}$ and deuterium. The $\mathrm{P}-\mathrm{CD}_{2}$ groups should appear as a doublet of 1:2:3:2:1 quintets with a concomitant loss in intensity. Furthermore, the carbon atom is no longer protonated so nuclear Overhauser enhancement is absent.

The upfield shift of the ${ }^{13} \mathrm{C}$ resonance is consistent with reports of isotope-induced changes to ${ }^{13} \mathrm{C}\left\{{ }^{1} \mathrm{H}\right\}$ NMR spectra. ${ }^{148}$ 
$P N P+[\operatorname{Ir}(\operatorname{cod})(\mu-C l)]_{2}$

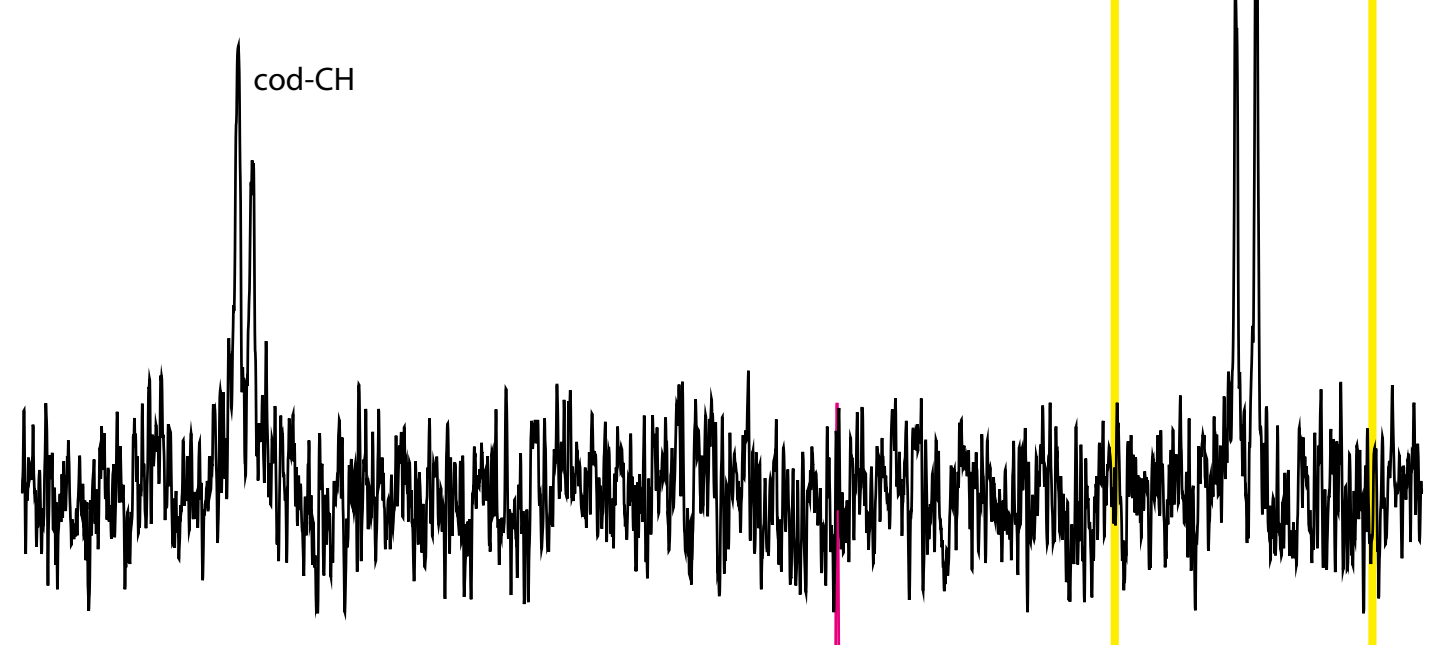

$P N P+[\operatorname{Ir}(\operatorname{cod})(\mu-O M e)]_{2}$

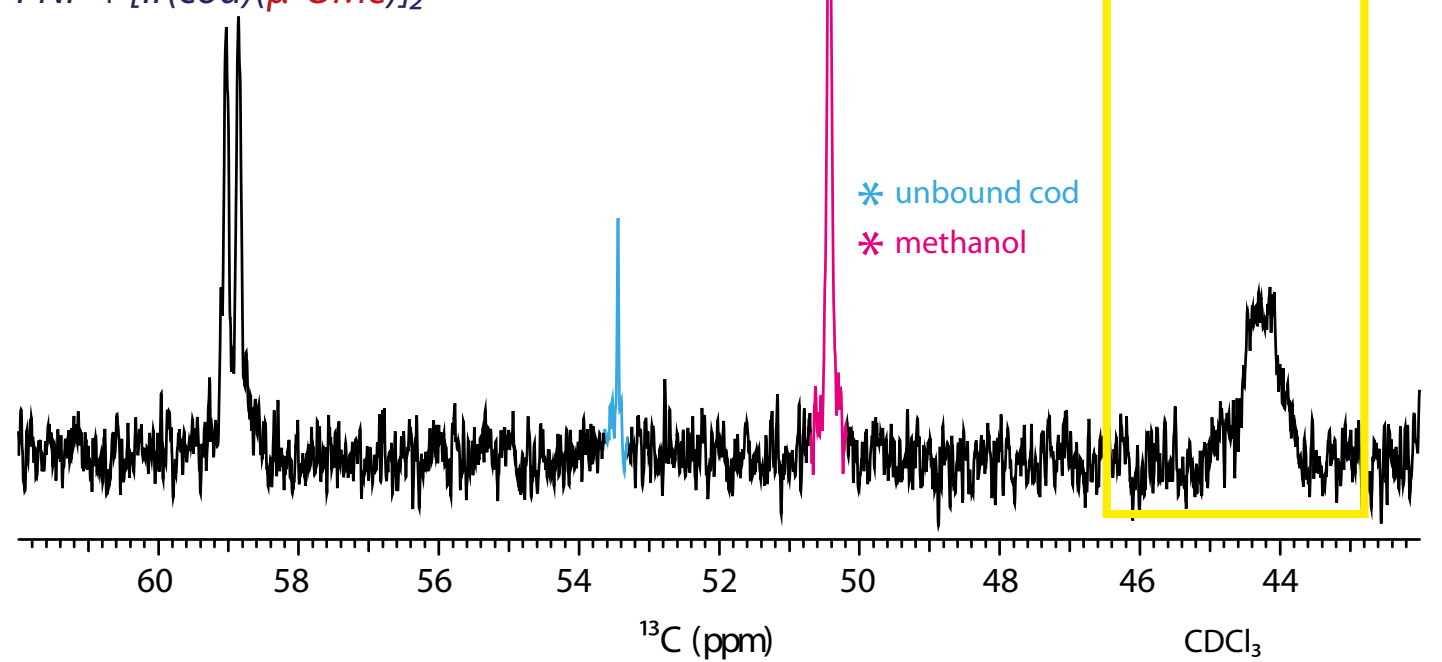

Figure 4.7 Change to the shape and position of the ${ }^{13} \mathrm{C}\left\{{ }^{1} \mathrm{H}\right\}$ NMR signal of $\mathrm{P}-\mathrm{CH}_{2}$ upon deuteration. 


\subsection{Reactivity of $\left[\left({ }^{2-p y r} \mathrm{PNP}\right) \operatorname{Ir}(\operatorname{cod})\right] \mathrm{Cl}$}

\subsubsection{Ethene}

Bis(alkenes) such as $\eta^{4}$-1,5-cyclooctadiene (cod) are often difficult to displace due to the chelate effect. This may have a detrimental effect on the catalytic activity of the complex. Thus, complex $\mathbf{3 3}$ was reacted with ethene in order to determine whether cod could be replaced with a more labile ligand.

When ethene was bubbled through a solution of $\mathbf{3 3}$ for a few minutes, or the solution stirred under an atmosphere of ethene, a new signal in the ${ }^{31} \mathrm{P}\left\{{ }^{1} \mathrm{H}\right\}$ NMR spectrum appeared $\left(\mathrm{CD}_{2} \mathrm{Cl}_{2}\right.$ : singlet at $\delta_{P} 4.0 \mathrm{ppm} ; 1: 1$ acetone- $\mathrm{d}_{6} / \mathrm{D}_{2} \mathrm{O}$ : multiple peaks at approximately $4.5 \mathrm{ppm})$. The appearance of the new signal in the ${ }^{31} \mathrm{P}\left\{{ }^{1} \mathrm{H}\right\} \mathrm{NMR}$ spectrum was accompanied by the appearance of free cod in the ${ }^{1} \mathrm{H}$ NMR spectrum and was ascribed to the formation of the novel bis(ethene) complex 36 (Scheme 4.4). Full conversion as determined by ${ }^{31} \mathrm{P}\left\{{ }^{1} \mathrm{H}\right\}$ NMR occurred over a period of weeks in either solvent. To reduce the reaction time, the solution of $\mathbf{3 3}$ was placed under 5 atm ethene. After two days at room temperature, the ${ }^{31} \mathrm{P}\left\{{ }^{1} \mathrm{H}\right\}$ NMR spectrum showed complete conversion to $\mathbf{3 6}$. The identity of this complex was confirmed by analysing the ${ }^{1} \mathrm{H},{ }^{31} \mathrm{P}$, and ${ }^{13} \mathrm{C}$ NMR spectra.

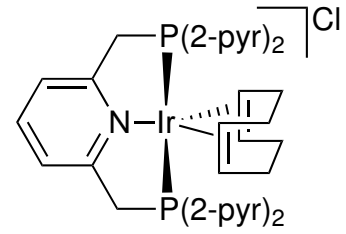

33

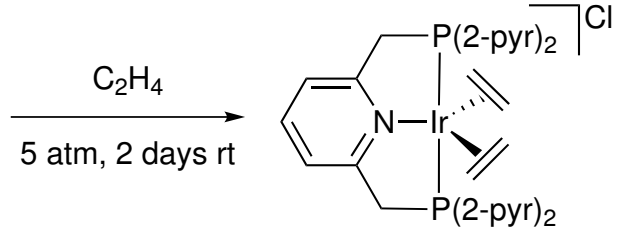

36

Scheme 4.4 Synthesis of bis(ethene) complex 36 .

The ${ }^{1} \mathrm{H}$ and ${ }^{13} \mathrm{C}\left\{{ }^{1} \mathrm{H}\right\}$ NMR spectra show that $\mathbf{3 6}$ has a higher degree of symmetry than 33. All four pyridyl rings are equivalent, and the $\mathrm{P}-\mathrm{CH}_{2}$ resonance is a virtual triplet due to strongly coupled trans phosphorus atoms. This coupling is not present for 33 due to the facial coordination of ${ }^{2-p y r} \mathrm{PNP}$. The ${ }^{1} \mathrm{H},{ }^{31} \mathrm{P}$, and ${ }^{13} \mathrm{C}$ NMR spectra are solvent-dependent, and the ${ }^{1} \mathrm{H}$ and ${ }^{13} \mathrm{C}$ spectra are temperature-dependent.

\section{Solvent}

Complex 36 was synthesised in both $\mathrm{CD}_{2} \mathrm{Cl}_{2}$ and a $1: 1$ mixture of acetone- $\mathrm{d}_{6} / \mathrm{D}_{2} \mathrm{O}$. In dichloromethane a pale brown solid formed as the reaction progressed. This solid could not be dissolved in any of the solvents (or solvent mixtures) that are able to 
solubilise the ${ }^{2-p y r} \mathrm{PNP}$-containing complexes described in this work. In contrast, the reaction that was carried out in acetone- $\mathrm{d}_{6} / \mathrm{D}_{2} \mathrm{O}$ did not form any such byproducts.

Whereas the ${ }^{31} \mathrm{P}\left\{{ }^{1} \mathrm{H}\right\}$ NMR spectrum remained a singlet in $\mathrm{CD}_{2} \mathrm{Cl}_{2}$, the spectrum in acetone- $\mathrm{d}_{6} / \mathrm{D}_{2} \mathrm{O}$ changed over time. Figure 4.8 shows the change in the ${ }^{31} \mathrm{P}\left\{{ }^{1} \mathrm{H}\right\}$ NMR spectrum over 3 days as $\mathbf{3} 3$ reacted with the ethene that was dissolved in the solvent.

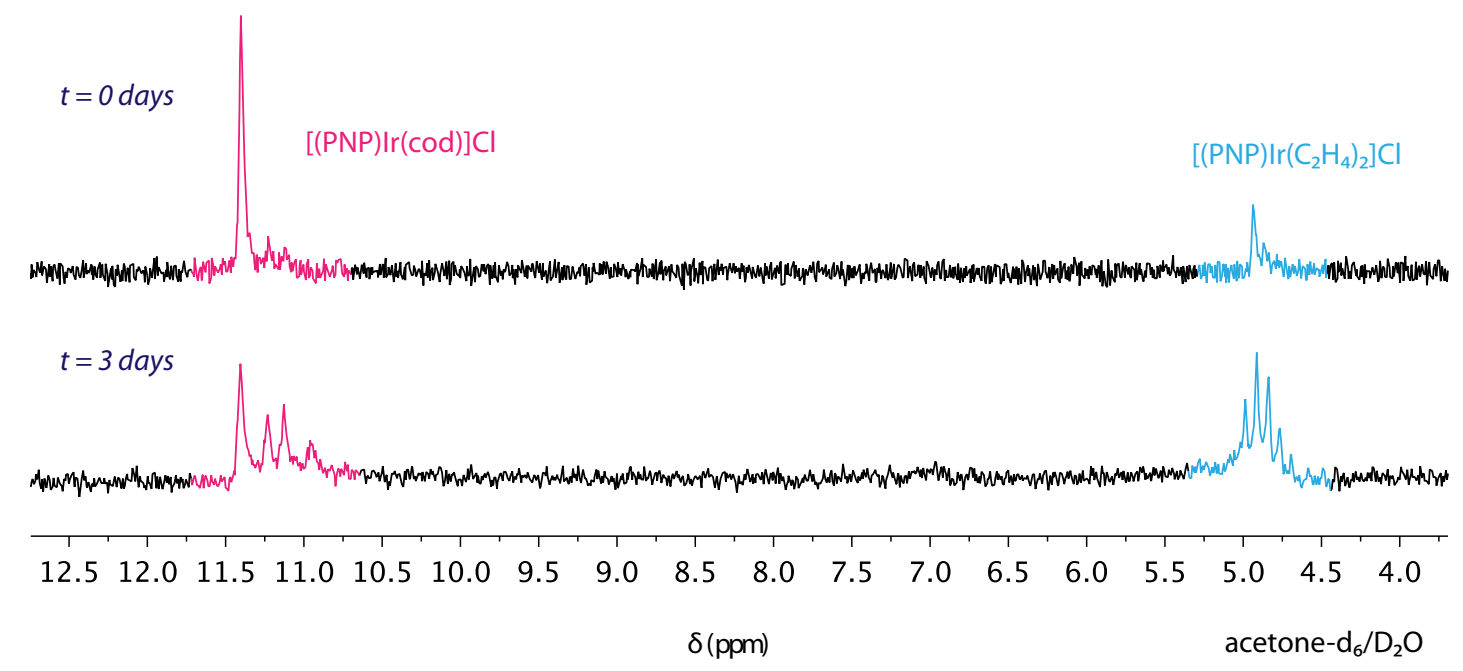

Figure 4.8 Change to ${ }^{31} \mathrm{P}\left\{{ }^{1} \mathrm{H}\right\}$ NMR spectrum.

In acetone- $\mathrm{d}_{6} / \mathrm{D}_{2} \mathrm{O}$, both $\mathbf{3 3}$ and $\mathbf{3 6}$ undergo slow deuteration at the $\mathrm{P}-\mathrm{CH}_{2}$ positions of the pincer ligand. This could be hastened by heating the samples and is reflected in the final ${ }^{31} \mathrm{P}\left\{{ }^{1} \mathrm{H}\right\}$ NMR spectrum as a number of closely-spaced signals. The complex was synthesised in acetone $/ \mathrm{H}_{2} \mathrm{O}$ to determine whether the ${ }^{31} \mathrm{P}\left\{{ }^{1} \mathrm{H}\right\}$ NMR spectrum remained a singlet; unfortunately, the resolution of the resulting NMR spectrum was too low to determine whether the closely-spaced singlets were present. Deuterium incorporation was also evident from a decrease in the integral for the $\mathrm{P}-\mathrm{CH}_{2}$ position from 4 to 0.72 protons.

\section{Temperature}

At $20{ }^{\circ} \mathrm{C}$, the ${ }^{1} \mathrm{H}$ NMR spectra of 36 in both solvents display signals for free cod, free ethene, a small amount of water, and the ${ }^{2-p y r}$ PNP ligand backbone. No signals for coordinated ethene are visible in either $\mathrm{CD}_{2} \mathrm{Cl}_{2}$ or acetone- $\mathrm{d}_{6} / \mathrm{D}_{2} \mathrm{O}$. However, in acetone- $\mathrm{d}_{6} / \mathrm{D}_{2} \mathrm{O}$ the baseline rises in a gentle curve near $2 \mathrm{ppm}$. A broad signal at this chemical shift suggests that although ethene ligands have coordinated to the metal, they are rotating about the metal-ligand axis on a similar timescale to the NMR experiment. Thus, NMR spectra were recorded at different temperatures to 
investigate this process and to determine the number of ethene molecules coordinated to iridium.

Over the range of temperatures measured, the ${ }^{31} \mathrm{P}\left\{{ }^{1} \mathrm{H}\right\}$ NMR spectra did not change in either solvent. This suggests that the dynamic process is limited to the ethene ligands. The ${ }^{1} \mathrm{H}$ and ${ }^{13} \mathrm{C}\left\{{ }^{1} \mathrm{H}\right\}$ NMR spectra changed with temperature in the regions where coordinated ethene signals are expected. The signal for free ethene remained sharp at every temperature.

Figure 4.9 shows the ${ }^{1} \mathrm{H}$ NMR spectra collected at temperatures between 40 and $-70{ }^{\circ} \mathrm{C}$ in $\mathrm{CD}_{2} \mathrm{Cl}_{2}$. At $40{ }^{\circ} \mathrm{C}$, a broad signal appeared at $2 \mathrm{ppm}$. Due to the temperature limit imposed by the boiling point of $\mathrm{CD}_{2} \mathrm{Cl}_{2}$, this signal could not be investigated further. However, the low melting point of $\mathrm{CD}_{2} \mathrm{Cl}_{2}\left(-96{ }^{\circ} \mathrm{C}\right)$ made it ideal for investigating the low temperature behaviour of $\mathbf{3 6}$. As the temperature was lowered, broad signals began to appear at 2.74 and $1.12 \mathrm{ppm}$. At $-70{ }^{\circ} \mathrm{C}$ the signals had sharpened such that integration could be carried out. Integration of each signal for four protons confirmed that two ethene ligands had replaced the cod ligand. The coalescence temperature for the dynamic process is near $20{ }^{\circ} \mathrm{C}$. 


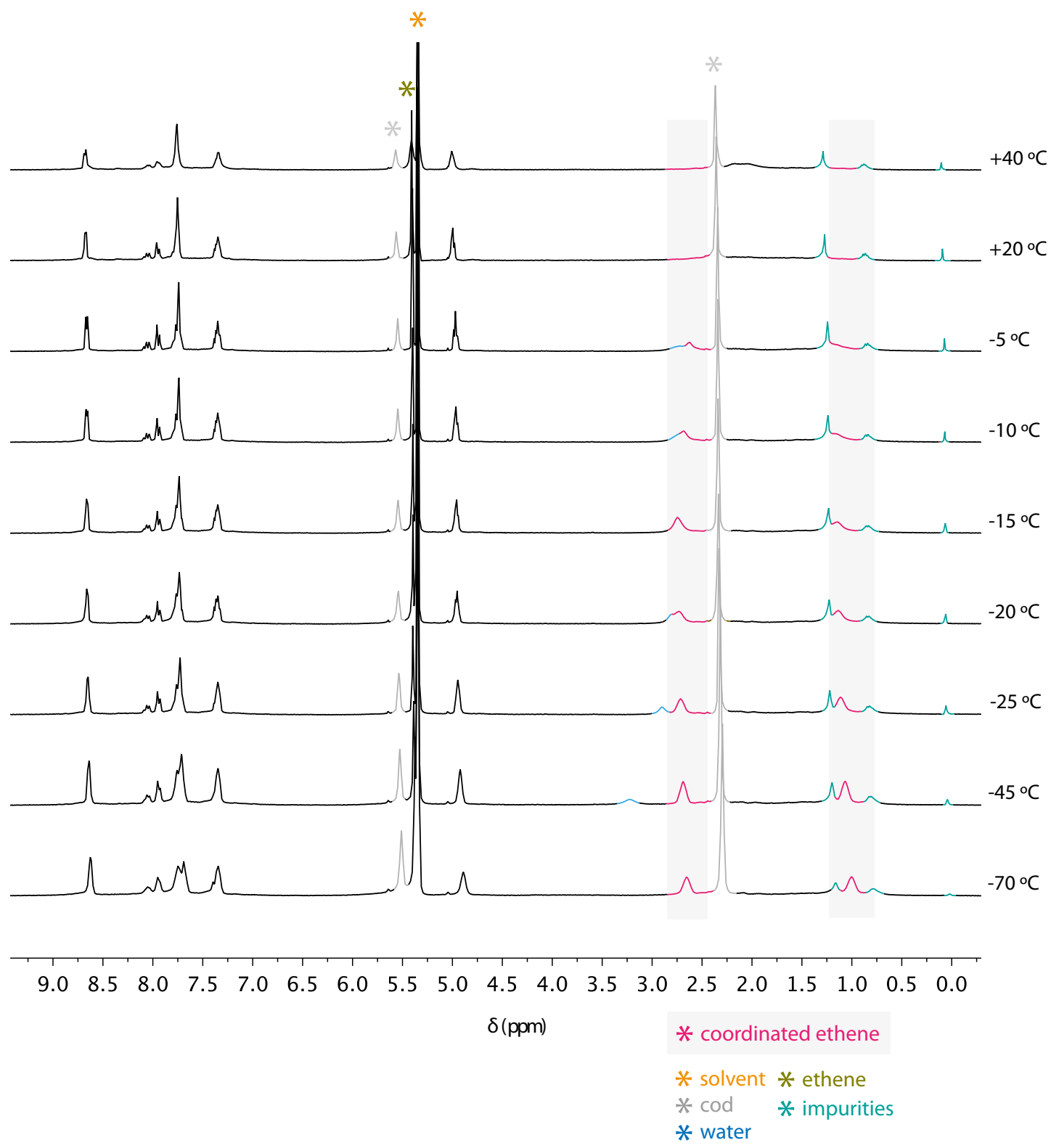

Figure 4.9 ${ }^{1} \mathrm{H}$ NMR spectra of $\mathbf{3 6}$ collected at temperatures between +40 and $-70{ }^{\circ} \mathrm{C}$ in $\mathrm{CD}_{2} \mathrm{Cl}_{2}$. 
The presence of water in the ${ }^{1} \mathrm{H}$ NMR spectra is likely due to the method used to synthesise 36. The ethene was not dried prior to pressurising the Fisher-Porter tube, and only about $0.6 \mu \mathrm{L}$ water would be enough to introduce the amount observed in the NMR spectrum.

NMR spectra of 36 collected between 50 and $-20{ }^{\circ} \mathrm{C}$ in $1: 1$ acetone- $\mathrm{d}_{6} / \mathrm{D}_{2} \mathrm{O}$ are displayed in Figure 4.10. In this solvent mixture, the coalescence temperature is near $0{ }^{\circ} \mathrm{C}$. This is approximately $20{ }^{\circ} \mathrm{C}$ lower than in $\mathrm{CD}_{2} \mathrm{Cl}_{2}$ and implies that the ethene molecules are able to rotate faster at room temperature in acetone- $\mathrm{d}_{6} / \mathrm{D}_{2} \mathrm{O}$. Measurements were limited by the freezing point of water, but it is clear from comparing the spectra measured at $-20{ }^{\circ} \mathrm{C}$ in Figure 4.9 and Figure 4.10 that the low temperature limit is more difficult to reach in acetone- $\mathrm{d}_{6} / \mathrm{D}_{2} \mathrm{O}$ than in $\mathrm{CD}_{2} \mathrm{Cl}_{2}$. The spectra from $20{ }^{\circ} \mathrm{C}$ to $50{ }^{\circ} \mathrm{C}$ show the appearance of a signal at $\delta_{H} 2.0 \mathrm{ppm}$ due to the rapidly-rotating ethene ligands.

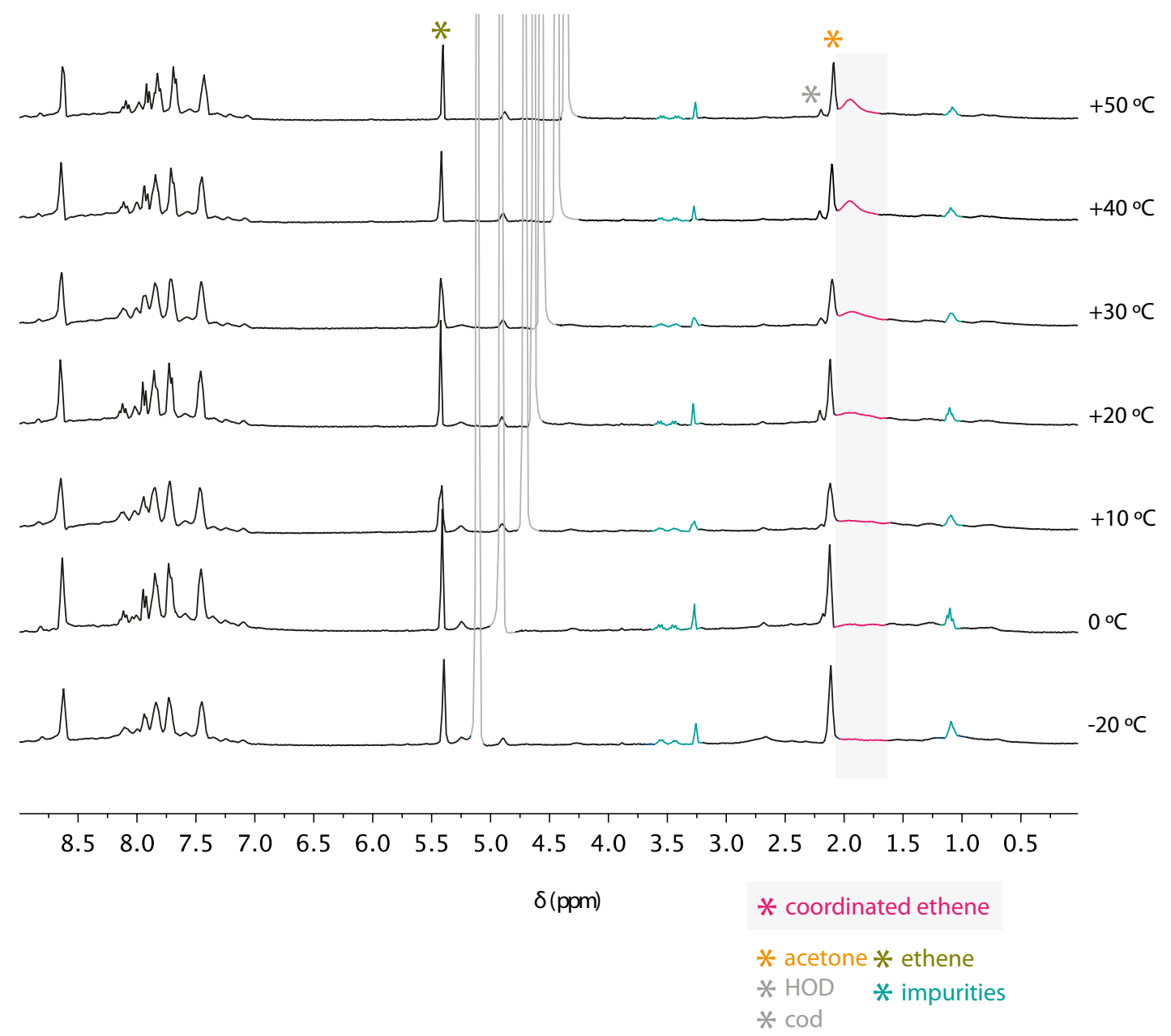

Figure 4.10 ${ }^{1} \mathrm{H}$ NMR spectra of $\mathbf{3 6}$ collected at temperatures between +50 and $-20{ }^{\circ} \mathrm{C}$ in $1: 1$ acetone- $\mathrm{d}_{6} / \mathrm{D}_{2} \mathrm{O}$. 


\section{Geometry of 36}

The coordination geometry of ${ }^{2-p y r}$ PNP changes from facial in cod complex 33 to meridional in bis(ethene) complex 36. This is shown in Figure 4.11, a simplified version of Scheme 4.4. This is due to the two ethene ligands being more flexible with regards to their coordination preferences. When cod coordinates as a chelating bis(alkene) it has a bite angle of approximately $90^{\circ}$. For a trigonal bipyramidal complex, this is best accommodated by taking up a position that spans the axial and equatorial positions. In 33, where the bite angle of cod in the X-ray crystal structure is $86.18^{\circ}$, this requirement forces ${ }^{2-p y r} \mathrm{PNP}$ to be facially coordinated.

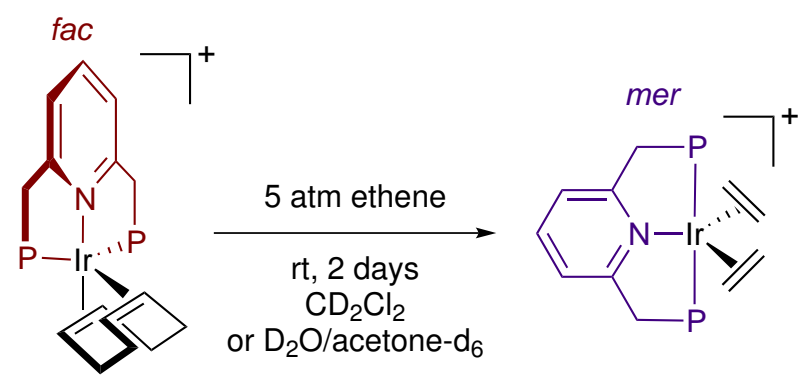

Figure 4.11 The pincer geometry changes when cod is displaced by ethene.

The variable temperature ${ }^{1} \mathrm{H}$ NMR study confirms the presence of two ethene ligands. However, it could not differentiate between structures where the ethene ligands are coordinated parallel or perpendicular to the trigonal bipyramidal plane. In order to do this, ${ }^{13} \mathrm{C}\left\{{ }^{1} \mathrm{H}\right\}$ NMR spectra were required (Figure 4.12). Upright ligands would show one signal at the low temperature limit, whereas ligands lying in the equatorial plane would show two. The absence of a signal at $5{ }^{\circ} \mathrm{C}$ is consistent with the extremely broad signal for the ethene ligands in the ${ }^{1} \mathrm{H}$ NMR spectrum recorded at the same temperature. At $-40{ }^{\circ} \mathrm{C}$, two signals appeared. This is evidence that the ethene ligands lie in the equatorial plane and contrasts with the axial-equatorial arrangement in the only other bis(ethene) pincer complex $\left[\left({ }^{\mathrm{CF}_{3} \mathrm{PCP}}\right) \operatorname{Ir}\left(\mathrm{C}_{2} \mathrm{H}_{4}\right)_{2}\right] .{ }^{144}$ Pyridyl substituents are less electron-withdrawing than $\mathrm{CF}_{3}$ substituents, so ${ }^{2-p y r} \mathrm{PNP}$ cannot exert as great an influence on the electronic properties of the iridium(I) centre; thus, the ethene ligands in $\mathbf{3 6}$ will take up the equatorial positions where there is more favourable overlap between the $\pi^{*}$ orbitals of the ethene ligands and the $\mathrm{d} \pi$ orbitals of the metal. ${ }^{149}$ 


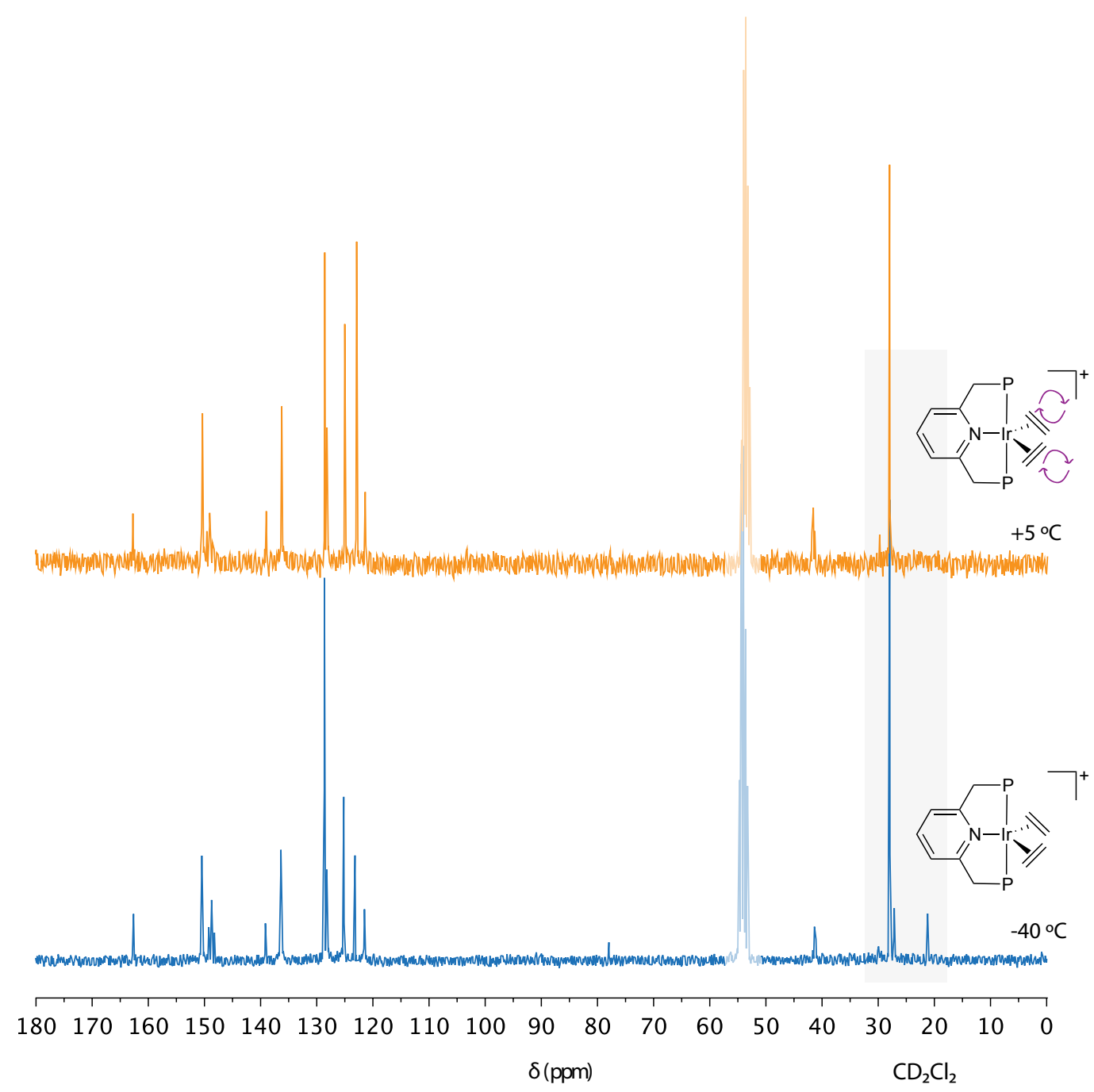

Figure 4.12 ${ }^{13} \mathrm{C}\left\{{ }^{1} \mathrm{H}\right\}$ NMR spectra of 36 collected at $+5{ }^{\circ} \mathrm{C}$ and $-40{ }^{\circ} \mathrm{C}$ in $\mathrm{CD}_{2} \mathrm{Cl}_{2}$. 
Complex 36 is the first example of a bis(monoalkene) iridium PNP complex. Nozaki et al. have reported the sole example of an iridium PNP ethene complex. ${ }^{76}$ This square planar 16 electron complex was generated by reductive elimination of a metallated neopentyl group (see Figure 4.1) in the presence of ethene and was characterised by NMR spectroscopy. Three ethene complexes have been reported with $\mathrm{CF}_{3} \mathrm{PCP}$

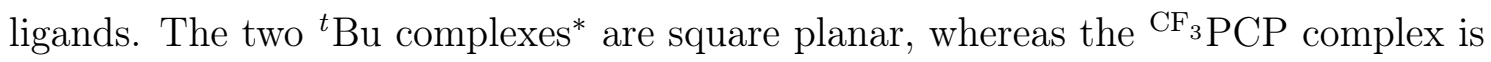
five-coordinate. Like the cod complex discussed previously, $\left[\left({ }^{\mathrm{CF}_{3} \mathrm{PCP}}\right) \operatorname{Ir}\left(\mathrm{C}_{2} \mathrm{H}_{4}\right)_{2}\right]$ has a distorted trigonal bipyramidal geometry. ${ }^{150}$

The method used to synthesise $\mathbf{3 6}$ results in free cod remaining in solution. This has a high boiling point so could not be removed under reduced pressure without resulting in decomposition of 36 . Preliminary attempts at crystallisation by slow diffusion of diethyl ether into a $\mathrm{CD}_{2} \mathrm{Cl}_{2}$ solution at $-18{ }^{\circ} \mathrm{C}$ did not yield a crystalline material. When 36 was synthesised in 1:1 acetone- $\mathrm{d}_{6} / \mathrm{D}_{2} \mathrm{O}$ and left in a sealed Schlenk tube at $4{ }^{\circ} \mathrm{C}$ for 1 month, 36 converted back to 33 . As the ethene diffused out of solution, the equilibrium shifted to replace the ethene in solution. In doing so, this may form a coordinatively unsaturated complex. In the presence of free cod, this would quickly react to form 33; metal complexes with chelating ligands typically have a higher formation constant because of the entropy increase that results when monodentate ligands are displaced by a multidentate ligand.

\subsubsection{Hydrogenation of $\left[\left({ }^{2-p y r} \mathrm{PNP}\right) \operatorname{Ir}(\operatorname{cod})\right] \mathrm{Cl}$}

Compound 33 was reacted with hydrogen in order to determine whether the cod ligand could be replaced with hydride ligands. Transition metal hydride complexes are extremely versatile, as they are able to lose hydrogen in a number of ways to generate coordinatively unsaturated species. These include through reaction with a donor molecule, thermal decomposition, and protonation. ${ }^{151}$ Such complexes are also able to enter catalytic cycles by insertion of a reactant into an $\mathrm{Ir}-\mathrm{H}$ bond.

Iridium PCP hydrido complexes are highly active in acceptorless alkane dehydrogenation and alkane metathesis. ${ }^{53}$ Nozaki's $\left[\left({ }^{t} \mathrm{Bu} \mathrm{PNP}\right) \operatorname{Ir}(\mathrm{H})_{3}\right]$ complex, synthesised from the corresponding chloroiridium(III) di(hydride) complex, is extremely active in the catalytic hydrogenation of $\mathrm{CO}_{2} \cdot{ }^{137}$ The proposed mechanism involves a rate-determining dearomatisation step. ${ }^{152}$

Complex $\left[\left({ }^{2-p y r} \mathrm{PNP}\right) \operatorname{Ir}(\mathrm{cod})\right] \mathrm{Cl}(\mathbf{3 3})$ was dissolved in dichloromethane and placed

${ }^{*}$ Both ligands have ${ }^{t} \mathrm{Bu}$ groups attached to phosphorus but one of the ligands has a $-\mathrm{OP}\left({ }^{t} \mathrm{Bu}\right)_{2}$ group at the 4-position of the meta-xylene backbone 
under 5 atm hydrogen. After five days, the NMR signals of $\mathbf{3 3}$ had disappeared and air-stable chloroiridium(III) di(hydride) complex 37 had formed (Scheme 4.5). The reaction conditions employed in this work differ from the forcing conditions employed by Nozaki et al. (24.7 atm $\mathrm{H}_{2}$ at $90{ }^{\circ} \mathrm{C}$ in THF); however, the reaction described here also took 10 times longer (Scheme 4.5).

The NMR data of $\mathbf{3 7}$ are in accord with those of $\left[\left({ }^{t} \mathrm{Bu} \mathrm{PNP}\right) \operatorname{Ir}(\mathrm{H}){ }_{2} \mathrm{Cl}\right] .{ }^{137}$ The ${ }^{31} \mathrm{P}\left\{{ }^{1} \mathrm{H}\right\}$ NMR chemical shift change from free ${ }^{2-p y r}$ PNP to 37 was similar to that observed by Nozaki et al. $\left(\Delta \delta_{P} 20.2\right.$ vs $\left.22.01 \mathrm{ppm}\right)$.

Nozaki et al.
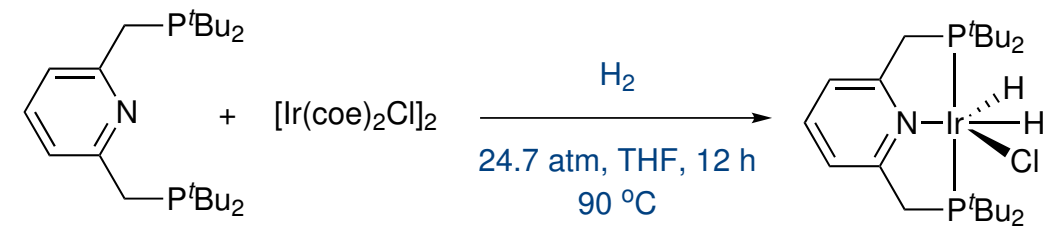

This work

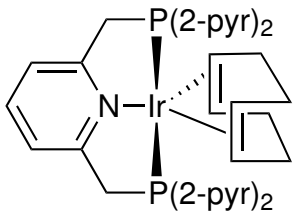

33

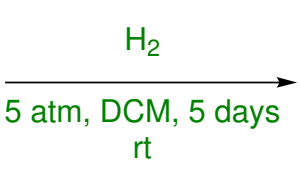

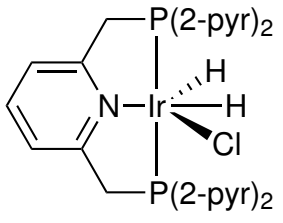

37

Scheme 4.5 Synthesis of 37 .

${ }^{1} \mathrm{H}$ NMR data confirm complex 37 has an octahedral geometry with cis hydride ligands and meridional coordination of ${ }^{2-p y r} \mathrm{PNP}$. The hydride resonances appeared as triplets of doublets at $\delta_{H}-18.34$ and $-21.56 \mathrm{ppm}$. The chemical shifts and coupling patterns of these resonances rule out the formation of a tri(hydride) complex and the presence of trans hydrides. Furthermore, a hydride signal near $-18 \mathrm{ppm}$ is indicative of a hydride trans to a chloride and cis to two identical phosphorus atoms. ${ }^{136}$ Facial coordination of the pincer ligand would not allow a trans relationship between $\mathrm{Cl}^{-}$ and $\mathrm{H}^{-}$to occur. In $\mathrm{CDCl}_{3}$, the $\mathrm{P}-\mathrm{CH}_{2}$ protons appear as an $\mathrm{AB}$ system of virtual triplets due to the $\mathrm{C}_{S}$ symmetry of the complex. In $\mathrm{CD}_{2} \mathrm{Cl}_{2}$, the $\mathrm{AB}$ system collapses into a virtual triplet.

The IR spectrum of $\mathbf{3 7}$ confirmed the positions of the hydride ligands. Ir-H stretches were observed at $2083 \mathrm{~cm}^{-1}$ and $2180 \mathrm{~cm}^{-1}$. These are similar to those reported for $\left[\left({ }^{t} \mathrm{Bu} \mathrm{PNP}\right) \operatorname{Ir}(\mathrm{H}){ }_{2} \mathrm{Cl}\right]\left(2097 \mathrm{~cm}^{-1}\right.$ and $\left.2303 \mathrm{~cm}^{-1}\right)$. The presence of a slightly weaker Ir-H bond in 37 is curious. A difference of $123 \mathrm{~cm}^{-1}$ may be significant, particularly given that pyridyl nitrogens can interact with hydride ligands. ${ }^{153}$ Further data from computational modelling and nOe experiments are required to investigate this further. 
A $50 \%$ yield of $\left[\left({ }^{\mathrm{pyr}} \mathrm{PNP}\right) \operatorname{Ir}(\mathrm{H})_{2} \mathrm{Cl}\right]$ was obtained. The remainder of the material was present as an orange-brown byproduct that could not be dissolved in any solvent or combination of solvents. An infrared spectrum showed the presence of $\mathrm{Ir}-\mathrm{H}$ bonds as a broad signal at $2160 \mathrm{~cm}^{-1}$.

Following the procedure of Nozaki et al., preliminary investigations into the chemistry of 37 involved reacting it with excess sodium hydride in an attempt to form the tri(hydride) species. After six hours at room temperature, no reaction between $\mathbf{3 7}$ and the $\mathrm{NaH}$ had occurred. Employing a more reactive hydride source such as KH, with or without addition of a crown ether, may improve this reaction.

\subsection{Concluding Remarks}

The synthesis of iridium ${ }^{2-p y r} \mathrm{PNP}$ complexes was investigated by reacting ${ }^{2-p y r} \mathrm{PNP}$ with three iridium(I) precursors. It was hoped that these would display much more straightforward reactivity with ${ }^{2-p y r}$ PNP than the rhodium complexes described in chapter three. This was indeed the case when ${ }^{2-p y r} \mathrm{PNP}$ was reacted with $[\operatorname{Ir}(\operatorname{cod})(\mu-$ $\mathrm{Cl})]_{2}$ : ${ }^{2-p y r} \mathrm{PNP}$ displaced $\mathrm{Cl}^{-}$immediately to form five-coordinate pincer complex 33. The reactivity of ${ }^{2-p y r} \mathrm{PNP}$ with iridium complexes bearing cyclooctene ligands is complicated.

Complex 33 displays trigonal bipyramidal geometry, and the unusual facial coordination of the pincer ligand was confirmed by X-ray crystallography. To investigate whether cod could be replaced with more labile ligands, 33 was reacted with ethene to form bis(ethene) complex 36. NMR data of the resulting complex establish that ${ }^{2-p y r} \mathrm{PNP}$ is a rare example of a PNP pincer ligand that is able to take on both meridional and facial geometries. Such flexibility is important for a catalyst as it places fewer constraints on the geometries available throughout the catalytic cycle. VT NMR studies of $\mathbf{3 6}$ were carried out in order to investigate the rotation of the ethene ligands around the ligand-metal axis. The data confirm that two ethene ligands are coordinated and that they lie planar at the low temperature limit.

Complexes 33 and $\mathbf{3 6}$ are sparingly soluble in water and much more soluble in waterrich acetone. Both complexes undergo deuterium incorporation at the methylene positions in hot water or in the presence of methoxide and chloroform. Such reactivity reveals certain benefits and limitations to employing the ${ }^{2-p y r} \mathrm{PNP}$ ligand: deprotonation suggests that dearomatisation/rearomatisation reactions are possible. However, ${ }^{2-p y r} \mathrm{PNP}$ is not soluble in the aprotic solvents that allow the dearomatised 
species to be isolated and studied. The suitability of dichloromethane must be investigated further.

Hydrogenation of $\mathbf{3 3}$ was investigated as a way of removing the cod ligand and replacing it with hydride ligands. Hydride complexes are often effective pre-catalysts. This gave chloroiridium(III) di(hydride) complex 37 in good yield. Complex 37 is octahedral with ${ }^{2-p y r}$ PNP occupying meridional positions and cis hydride ligands. It is much less stable in water than either $\mathbf{3 3}$ or $\mathbf{3 6}$.

\subsection{Future Work}

Three novel pincer complexes, each with two potentially "cooperative" features, have been synthesised. Thus, there exists much more chemistry that could be carried out in order to fully investigate the benefit of combining the PNP backbone with 2-pyridyl substituents.

The ability to study the influence of ${ }^{2-p y r} \mathrm{PNP}$ on the chemistry of iridium pincer complexes will benefit from an understanding of the basicity of the pyridyl nitrogens. For example, knowledge of their pKa values could inform a study of the change in the ${ }^{15} \mathrm{~N}$ NMR data upon protonation. Proton-responsive iridium complexes have been shown to be highly active in the hydrogenation of $\mathrm{CO}_{2}$, and the activities of nitrile hydration catalysts are often $\mathrm{pH}$ dependent. ${ }^{23,154}$

Particularly relevant to the synthesis of catalytically active complexes would be the optimisation of the anion metathesis reactions. Metal complexes without $\mathrm{Cl}^{-}$anions are often more active (pre-)catalysts. Nozaki et al. discovered that the tri(hydride) complex $\left[\left({ }^{t} \mathrm{Bu} \mathrm{PNP}\right) \operatorname{Ir}(\mathrm{H})_{3}\right]$ was much more active in the catalytic hydrogenation of $\mathrm{CO}_{2}$ than its chloroiridium(III) di(hydride) analogue. ${ }^{137}$ Compound 37 should therefore be treated with a hydride source in order to synthesise the analogous tri(hydride) complex. Hydrogenation of the $\mathrm{BArF}^{-}$or $\mathrm{BPh}_{4}^{-}$analogue of $\mathbf{3 3}$ would also be interesting, as the third coordination site would no longer be filled with a chlorido ligand. This may result in a shorter synthesis of $\left[\left({ }^{2-p y r} \mathrm{PNP}\right) \operatorname{Ir}(\mathrm{H})_{3}\right]$ or the formation of a five-coordinate di(hydride) complex. Such a complex complex would resemble the ${ }^{t}$ Bu PCP di(hydride) complex that has shown extremely high activity in alkane metathesis and acceptorless dehydrogenation reactions. ${ }^{53}$

Further work could also involve protonation of ${ }^{2-p y r} \mathrm{PNP}$ hydride complexes. This reaction has been used to synthesise dihydrogen complexes; however, with a ${ }^{2-p y r} \mathrm{PNP}$ 
ligand, further reactivity can be envisaged. For example, interactions between protonated nitrogen atoms on $\mathrm{Ph}_{2} \mathrm{P}(2$-pyr) and ruthenium hydride ligands have been observed by low temperature NMR studies. ${ }^{153}$ Protonation in the presence of alkenes may allow new $\eta^{2}$-alkene complexes to be formed by opening up a coordination site through loss of $\mathrm{H}_{2}$.

Investigating the acidity of the $\mathrm{P}-\mathrm{CH}_{2}$ positions would result in an increased understanding of the dearomatisation/rearomatisation reaction. Although reaction of $[\operatorname{Ir}(\operatorname{cod})(\mu-\mathrm{OMe})]_{2}$ with ${ }^{2-p y r} \mathrm{PNP}$ in chloroform resulted in $\mathrm{H} / \mathrm{D}$ exchange due to the acidic nature of the solvent, the reactivity of the $\mathrm{P}-\mathrm{CH}_{2}$ positions should be investigated under different conditions. For example, 33 could be reacted with a suitable base, followed by the addition of a substrate with an $\mathrm{H}-\mathrm{E}$ bond under solventfree conditions. Ideally, the base would deprotonate the ligand at the methylene position, leading to dearomatisation and a reactive position on the ligand. Subsequent rearomatisation through proton abstraction from $\mathrm{H}-\mathrm{E}$ would result in the desired $\mathrm{H}-\mathrm{E}$ activation reaction. 


\section{Chapter 5}

\section{Catalysis}

Few catalytic reactions have been reported using iridium PNP complexes. These are limited to the hydrogenations of carbon dioxide and levulinic acid. ${ }^{137,155}$ Furthermore, neither alkyne nor nitrile hydration has been carried out using a pincer complex. Therefore, there is scope to investigate whether either reaction can be carried out using such a complex, particularly one bearing groups with the potential for cooperativity.

As discussed in chapter 1, ${ }^{2-p y r} \mathrm{PNP}$ could participate in a hydration reaction in numerous ways. As an example, Figure 5.1 shows a suggested catalytic cycle for the hydration of a terminal alkene using $\left[\left({ }^{2-\mathrm{pyr}} \mathrm{PNP}\right) \operatorname{Ir}(\mathrm{H})_{2} \mathrm{Cl}\right]$ as the pre-catalyst.

Reductive elimination of $\mathrm{H}_{2}$ first generates an iridium(I) [(PNP)IrCl] species. In a basic reaction mixture, this is followed by dearomatisation. ${ }^{156}$ On reaction with water, the backbone rearomatises (Figure 5.1, C). ${ }^{51,156}$ Overall, steps A to C result in substitution of the chlorido ligand for a hydroxo ligand. After coordination of the alkene in step D, followed by 1,2-insertion in step E, the metal complex requires a hydrogen atom to be able to form an alcohol. By employing water as a source of hydrogen in step $\mathrm{F}$, its conjugate base $\left(\mathrm{OH}^{-}\right)$would be available to deprotonate the ligand. This oxidation reaction involves a metal-water interaction that was described in chapter one (Figure 1.4). ${ }^{31}$ The use of hydroxide to deprotonate the ligand is similar to a reaction reported in an alkyne hydration reaction (Figure 1.10). ${ }^{68}$ The final step, reductive elimination to form a $\mathrm{C}-\mathrm{H}$ bond, is a common metal-mediated reaction, and the chloride anion is able to reassociate with the metal to fill the vacant coordination site. ${ }^{40}$

As well as being able to participate in hydrogen bonding, pyridyl phosphines have been reported to deprotonate coordinated water. ${ }^{61,68}$ As shown in Figure 5.2, a tri(hydride) 


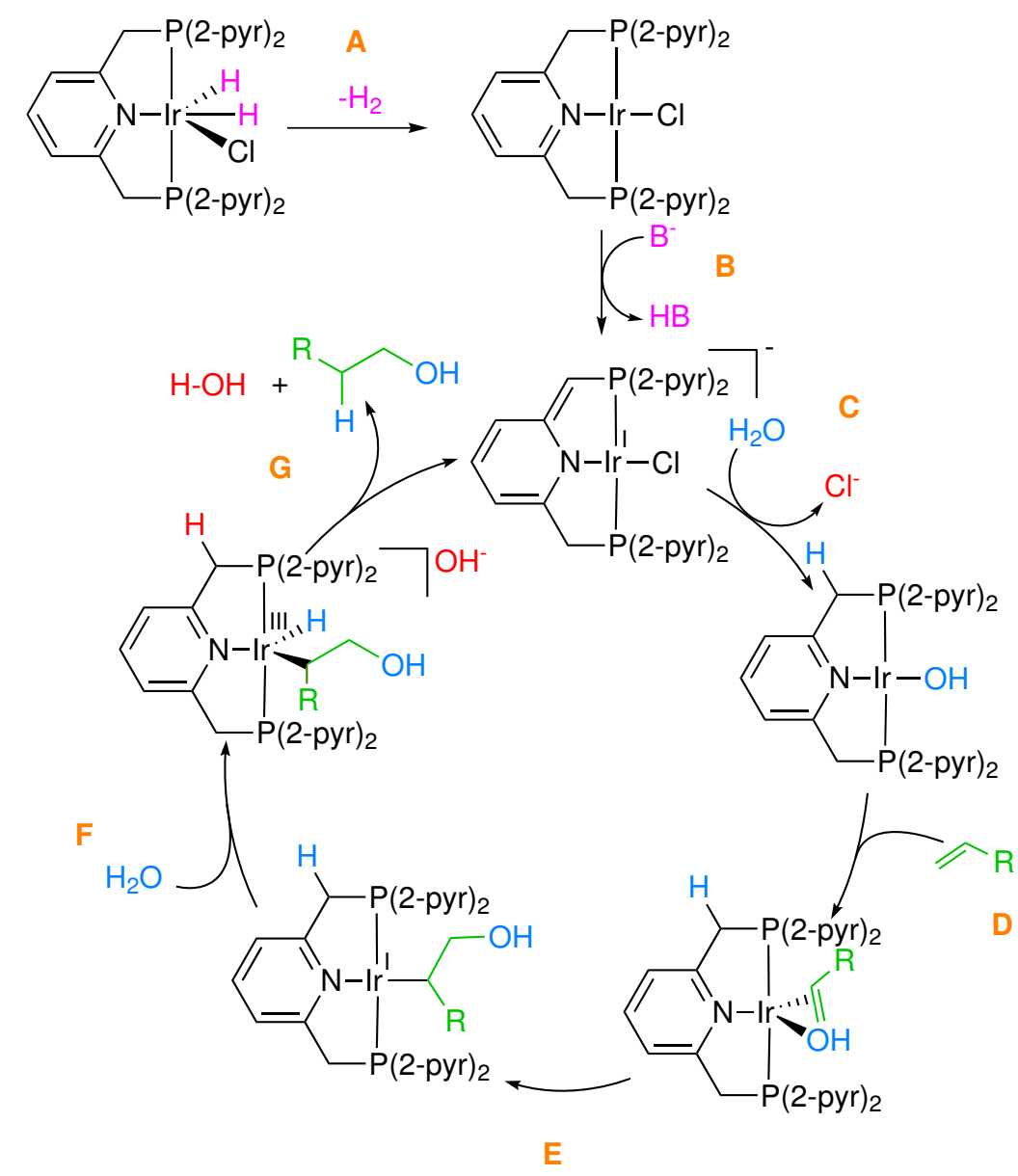

Figure 5.1 Hydration aided by the pincer backbone.

pre-catalyst could undergo reductive elimination of dihydrogen to generate a complex with vacant coordination sites. This cycle differs significantly from Figure 5.1 as steps A and B now act to generate an alkyl ligand, and it is only in step C that water is involved. Hydrogen bonding followed by a deprotonation reaction (similar to that observed by Grotjahn et al.) aids production of a hydroxo ligand in step C. ${ }^{68}$ Proton transfer to the metal and reductive elimination form the alcohol product in step E.

The ability of the iridium complexes synthesised in chapter four to hydrate alkene, terminal alkyne, and nitrile substrates in a water-rich reaction medium was investigated. Such reactions have not previously been realised using a pincer complex.

\subsection{Terminal Alkenes and Alkynes}

To determine whether cod complex 33 can add water across alkene and alkyne functional groups, six such substrates were combined with 33 (5 mol\%) in 1:1 acetone- $\mathrm{d}_{6} / \mathrm{D}_{2} \mathrm{O}$ in NMR tubes and heated at $70{ }^{\circ} \mathrm{C}$ for 16 hours. By this time, those containing substituted phenyl acetylenes $\left(4-{ }^{t} \mathrm{Bu}, 4-\mathrm{Me}\right)$, tert-butylacetylene, and 


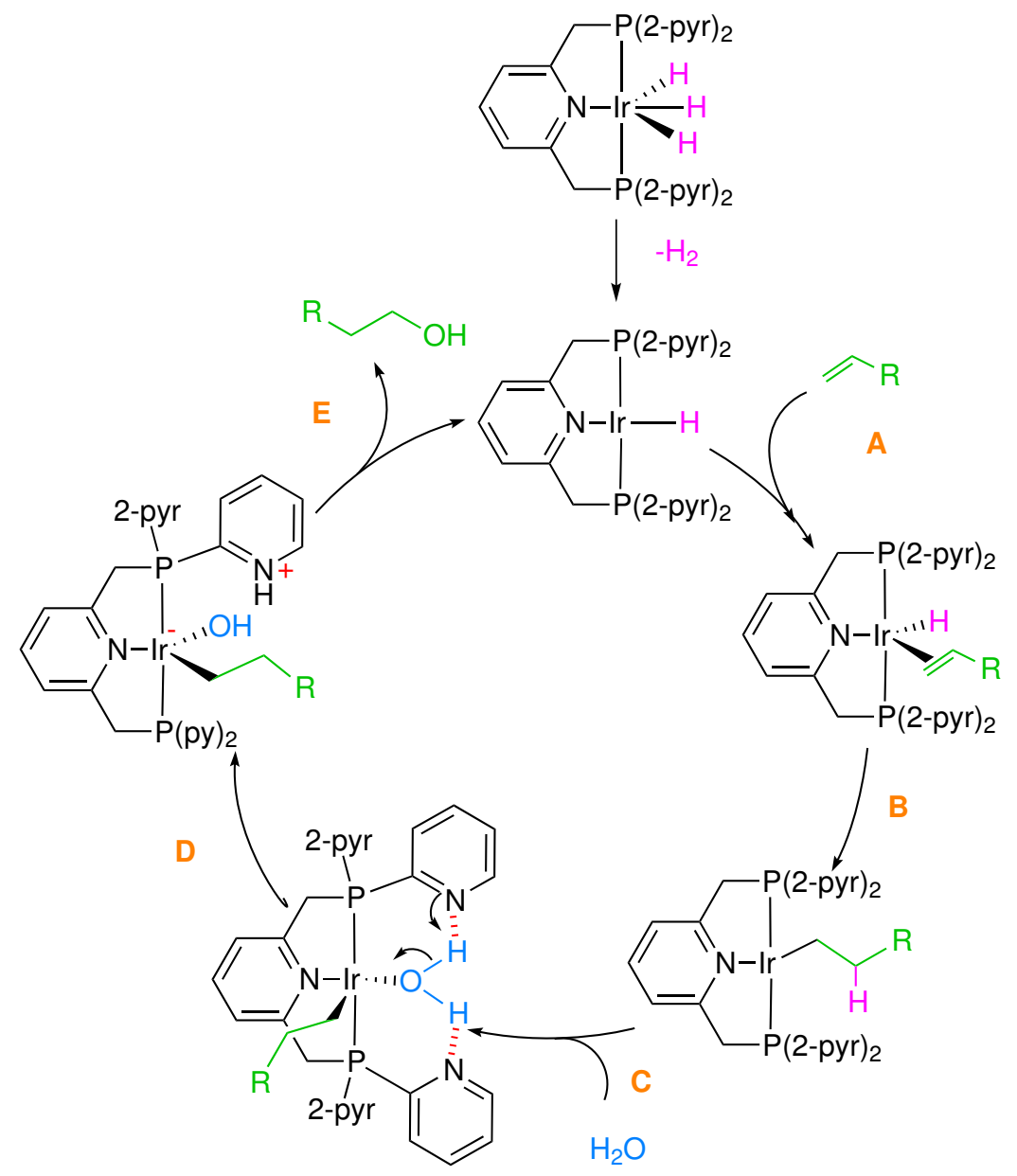

Figure 5.2 Hydration of a terminal alkene aided by the pyridyl substituents.

(triisopropylsilyl)acetylene were full of solid. Although the presence of unreacted substrates could still be observed by NMR, there were few distinctive signals in the aqueous solutions. Thus, each reaction was extracted into $\mathrm{CDCl}_{3}$ and $\mathrm{NMR}$ data collected. The resulting ${ }^{1} \mathrm{H}$ and ${ }^{13} \mathrm{C}$ NMR spectra contained new signals; yet, in almost all cases, the identities of the compounds responsible could not be determined using this information alone. This being said, it is unlikely that 1-hexene was hydrated as no signal between 3-5 ppm was observed. Such a signal would have appeared had a $\mathrm{CH}_{2}-\mathrm{OH}$ or $\mathrm{CH}-\mathrm{OH}$ group been present. Further investigation was not possible because the GC-MS was out of order.

When the reaction mixture containing (triisopropylsilyl)acetylene was extracted into chloroform and the ${ }^{1} \mathrm{H}$ and ${ }^{13} \mathrm{C}$ NMR spectra collected, distinctive signals near $\delta_{H} 6$ ppm were present. Corresponding signals in the ${ }^{13} \mathrm{C}\left\{{ }^{1} \mathrm{H}\right\}$ NMR spectrum suggested the alkyne had undergone head-to-head dimerisation, and comparison with the literature values for the enyne product $(E)$-1,4-bis(triisopropylsilyl)-1-buten-3-yne (Figure 5.3, 38) confirmed this hypothesis. ${ }^{157}$ It was difficult to confirm the presence of the $Z$-isomer as the NMR data for this compound have not been reported in the literature, and there are a number of overlapping signals in the NMR spectra. 


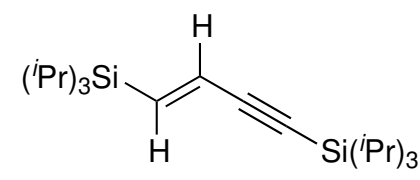

38

Figure 5.3 (E)-1,4-Bis(triisopropylsilyl)-1-buten-3-yne, $\mathbf{3 8}$.

The $\mathrm{C}_{\mathrm{sp}}-\mathrm{H}$ bond is much more reactive towards metals than other $\mathrm{C}-\mathrm{H}$ bonds, and the dimerisation of alkynes facilitated by metal complexes is well-established. Compound $\mathbf{3 8}$ has been synthesised using 8 different metals, ranging from calcium to palladium. ${ }^{157,158}$ However, none of these used an aqueous solvent system. ${ }^{157-164}$ Only one example used iridium to catalyse the dimerisation. This was carried out at $80{ }^{\circ} \mathrm{C}$ for 12 hours in a mixture of cyclohexane and $\mathrm{NEt}_{3}$, and a $50 \%$ yield of $\mathbf{3 8}$ was obtained (with $86 \%$ the $E$-isomer). ${ }^{160}$ GC-MS analysis would have provided the corresponding yield for the dimerisation observed in this work. However, analysis by NMR was the only technique available.

A catalytic cycle for the dimerisation by PNP complex 33 is proposed in Figure 5.4 and is based on a report of iridium-mediated dimerisation of phenylacetylene by a ${ }^{t}$ BuPCP complex. ${ }^{165}$

In Figure 5.4, cod is displaced by triisoproylsilyl acetylene to form, via an $\eta^{2}$ alkyne intermediate, the five-coordinate product of $\mathrm{C}-\mathrm{H}$ oxidative addition (39). The next step is a migratory insertion reaction with 2,1-regioselectivity. Goldman et al. studied this process and found that, in the ${ }^{t}$ Bu $\mathrm{PCP}$ system, the higher energy 2,1-insertion route led to the observed product. Although 1,2-insertion was found to be facile, computational studies confirmed that it led to a higher energy enyne complex that was unable to undergo $\mathrm{C}-\mathrm{C}$ reductive elimination. ${ }^{165}$

Following the insertion reaction shown in Figure 5.4, $\mathrm{C}-\mathrm{C}$ reductive elimination is followed by the formation of an enyne complex. The product is then released by reaction with (triisopropylsilyl)acetylene. 

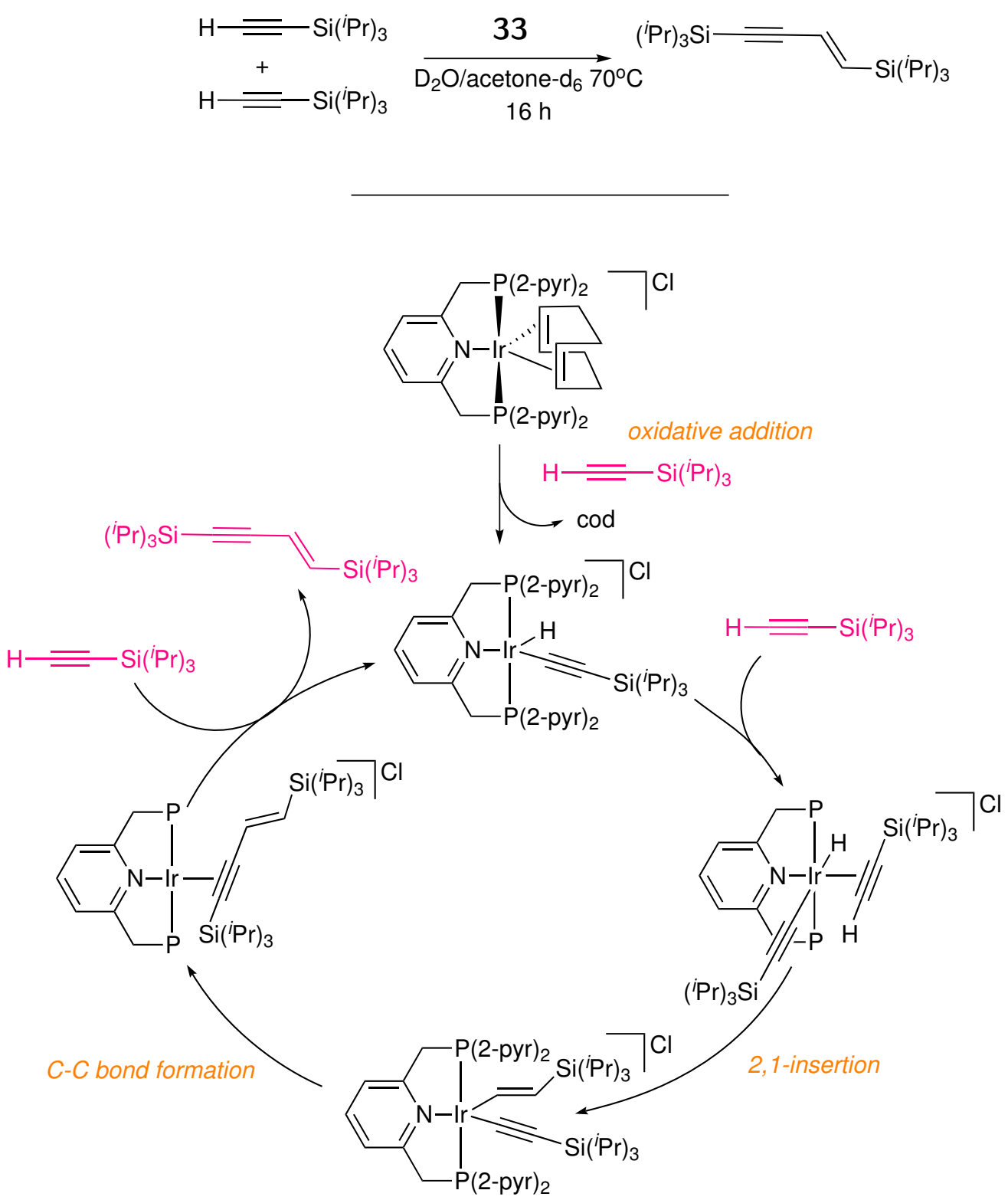

Figure 5.4 Proposed catalytic cycle for (triisopropylsilyl)acetylene dimerisation catalysed by 33 . $\mathrm{P}=\mathrm{P}(2-\mathrm{pyr})_{2}$. 


\subsection{Nitrile Hydration}

As discussed in chapter one, hydration of nitriles is an important and atom-economic method for the synthesis of primary amides. Such a process is particularly "green" if it can be carried out in water.

\subsubsection{Substrate Screening}

Initially, benzonitrile was reacted with $5 \mathrm{~mol} \%\left[\left({ }^{2-p y r} \mathrm{PNP}\right) \operatorname{Ir}(\operatorname{cod})\right] \mathrm{Cl}(33)$ and $\left[\left({ }^{2-p y r} \mathrm{PNP}\right) \operatorname{Ir}\left(\mathrm{C}_{2} \mathrm{H}_{4}\right)_{2}\right] \mathrm{Cl}(\mathbf{3 6})$ in $\mathrm{D}_{2} \mathrm{O}$ to determine if either complex was active at all. There are no reports of iridium pincer complexes catalysing nitrile hydration.<smiles>N#Cc1ccccc1C(=O)OCC(=O)c1ccccc1</smiles>

Scheme 5.1 Hydration of benzonitrile by $\mathbf{3 3}$ and $\mathbf{3 6}$ in $\mathrm{D}_{2} \mathrm{O}$ to form benzamide- $\mathrm{d}_{2}$.

Benzonitrile is only slightly soluble in water, and as the reaction progressed, the yellow reaction mixtures became cloudier. After 16 hours of heating, both samples were dried and the residues dissolved in $\mathrm{CDCl}_{3}$. The ${ }^{1} \mathrm{H}$ NMR spectra from both reactions show a single product whose NMR data match those of benzamide. The yields of these reactions could not be determined as the GC-MS remained out of order.

With confirmation that a nitrile could be hydrated by both pincer complexes, four more substrates were reacted under the same conditions. The results of these reactions are summarised in Table 5.1.

The greatest conversion was obtained with 2-cyanopyridine, with the remaining substrates hydrated very slowly. This may be due to increased electrophilicity of the nitrile carbon in 2-cyanopyridine. It would be interesting to investigate whether the heteroaromatic substrate gives a higher yield than benzonitrile, which could be achieved by carrying out a competition reaction. However, GC-MS analysis would be required to determine accurate yields. Acrylonitrile was hydrated by both complexes, but the vinylic resonances of the product overlap with those of the substrate, precluding determination of the yield by NMR. 
Table 5.1 Substrate screening. Hydration of nitriles RCN using $5 \mathrm{~mol} \%$ $\left[\left({ }^{2-p y r} \mathrm{PNP}\right) \operatorname{Ir}(\mathrm{cod})\right] \mathrm{Cl}(\mathbf{3 3})$ and $\left[\left({ }^{2-\mathrm{pyr}} \mathrm{PNP}\right) \operatorname{Ir}\left(\mathrm{C}_{2} \mathrm{H}_{4}\right)_{2}\right] \mathrm{Cl}(\mathbf{3 6})^{a}$

\begin{tabular}{ccccc} 
entry & $\mathbf{R}$ & time (h) & $\begin{array}{c}\text { yield }(\%)^{a} \\
\text { y3 }\end{array}$ & $\begin{array}{c}\text { yield }(\%)^{a} \\
\mathbf{3 6}\end{array}$ \\
\hline 1 & & & 48 & 66 \\
2 & $\mathrm{CH}_{2} \mathrm{CH}_{3}$ & 32 & 8 & 9 \\
3 & $\mathrm{CH}_{3}$ & 16 & 3 & 9 \\
4 & $\mathrm{CHCH}_{2}$ & 32 & $<10^{b}$ & $<10^{b}$ \\
\hline
\end{tabular}

${ }^{a}$ Reactions performed under argon in NMR tubes capped with septa with 0.1 mmol of each nitrile $\left(0.33 \mathrm{M}\right.$ in $\left.\mathrm{D}_{2} \mathrm{O}\right)$.

${ }^{a}$ Determined by NMR.

${ }^{b}$ Estimated due to overlapping resonances.

The ethene complex 36 is more active than complex 33 ; this is likely due to the presence of more labile ethene ligands.

\subsubsection{Temperature and Catalyst Loading}

After determining that both complexes are able to hydrate nitriles, the effects of temperature and catalyst loading on conversion were investigated. Heating at $60{ }^{\circ} \mathrm{C}$ for 32 hours did not result in complete conversion of any substrates, so temperatures below this were not investigated. 2-Cyanopyridine was used as the test substrate because its hydration product is soluble in $\mathrm{D}_{2} \mathrm{O}$.

The results are summarised in Table 5.2.

Entry 8 in Table 5.2 shows that 2-cyanopyridine can be hydrated in the absence of iridium. With 0.1 mol\% 36 (entry 7), background hydration is the only process occurring.

Increasing the temperature from $60{ }^{\circ} \mathrm{C}$ to $80{ }^{\circ} \mathrm{C}$ had a strong effect on conversion. A comparison between entries 2 and 3 shows that when the reaction was carried out at $80{ }^{\circ} \mathrm{C}$ for 3 hours, a $59 \%$ yield was observed, which only increased by $8 \%$ when the reaction was carried out for 32 hours at $60{ }^{\circ} \mathrm{C}$. Increasing the temperature to $100{ }^{\circ} \mathrm{C}$ increased the yield further. This temperature is classed as "relatively mild" for a nitrile hydration reaction, and many catalysts are tested at $100{ }^{\circ} \mathrm{C} .{ }^{22}$ 
Table 5.2 Reaction conditions. ${ }^{a}$

\begin{tabular}{cccccc} 
entry & catalyst & loading (\%) & temp $\left({ }^{\circ} \mathbf{C}\right)$ & time $(\mathbf{h})$ & Yield $_{(\%)}{ }^{a}$ \\
\hline 1 & $\mathbf{3 3}$ & 5 & 60 & 32 & 47 \\
2 & $\mathbf{3 6}$ & 5 & 60 & 32 & 67 \\
3 & $\mathbf{3 6}$ & 5 & 80 & 3 & 59 \\
4 & $\mathbf{3 3}$ & 5 & 100 & 3 & 58 \\
5 & $\mathbf{3 6}$ & 5 & 100 & 3 & 67 \\
6 & $\mathbf{3 6}$ & 1 & 100 & 3 & 24 \\
7 & $\mathbf{3 6}$ & 0.1 & 100 & 3 & 8 \\
8 & - & 0 & 100 & 3 & 12 \\
\hline
\end{tabular}

${ }^{a}$ Reactions performed under argon in NMR tubes capped with septa with 0.1 mmol of each nitrile $\left(0.33 \mathrm{M}\right.$ in $\left.\mathrm{D}_{2} \mathrm{O}\right)$.

${ }^{a}$ Determined by NMR.

Due to time constraints, nitrile hydration reactions were not investigated with $\left[\left({ }^{2-\text { pyr }} \mathrm{PNP}\right) \operatorname{Ir}(\operatorname{cod})\right] \mathrm{X}$ and $\left[\left({ }^{2-\text { pyr }} \mathrm{PNP}\right) \operatorname{Ir}\left(\mathrm{C}_{2} \mathrm{H}_{4}\right)_{2}\right] \mathrm{X}$ (where $\mathrm{X}$ is a weakly-coordinating anion). These complexes may be more active than their chloride analogues. ${ }^{125}$

\subsection{3 [( $\left.\left.{ }^{2-p y r} \mathrm{PNP}\right) \operatorname{Ir}(\mathrm{H})_{2} \mathrm{Cl}\right]$}

The iridium(III) complex [(2-pyr $\left.\mathrm{PNP}) \operatorname{Ir}(\mathrm{H})_{2} \mathrm{Cl}\right](37)$ was also reacted with nitriles in order to compare its reactivity with the two iridium(I) precursors. The results are summarised in Table 5.3.

Table 5.3 Hydration of nitriles RCN using $5 \mathrm{~mol} \%\left[\left({ }^{2-\mathrm{pyr}} \mathrm{PNP}\right) \operatorname{Ir}(\mathrm{H})_{2} \mathrm{Cl}\right] .{ }^{a}$

\begin{tabular}{ccc} 
entry & $\mathbf{R}$ & Yield $(\%)^{b}$ \\
\hline 1 & 2 -pyridyl & 66 \\
2 & $\mathrm{CH}_{2} \mathrm{CH}_{3}$ & 17 \\
3 & $\mathrm{CH}_{3}$ & 12 \\
4 & $\mathrm{CHCH}_{2}$ & $10-20^{c}$ \\
\hline
\end{tabular}

${ }^{a}$ Reactions performed at $100{ }^{\circ} \mathrm{C}$ for 6 hours in NMR tubes capped with septa with 0.1 mmol of each nitrile $\left(0.33 \mathrm{M}\right.$ in $\left.\mathrm{D}_{2} \mathrm{O}\right)$.

${ }^{b}$ Determined by NMR.

${ }^{c}$ Estimated due to overlapping resonances.

Again, some acrylonitrile was hydrated, but the yield could not be determined because the amide ${ }^{1} \mathrm{H}$ NMR signals overlap with those of the starting material. There was no evidence for hydration of the carbon-carbon double bond of acrylonitrile. 
Complex 37 is less active than $\left[\left({ }^{2-p y r} \mathrm{PNP}\right) \operatorname{Ir}(\operatorname{cod})\right] \mathrm{Cl}$. After 6 hours with cod complex 33, $99 \%$ conversion was achieved, compared to only $66 \%$ with $\mathbf{3 7}$. The hydride ligands may be less labile than the alkene ligands, preventing coordination of the nitrile.

\subsubsection{Pyridyl Groups}

Catalysts with hydrogen bond acceptors located near the active site have been used to increase the rates of hydration reactions (see chapter one, section 1.6.1). To determine whether the inclusion of pyridyl rings on the PNP ligand is beneficial in this case, 33 was compared with its ${ }^{\mathrm{Ph}} \mathrm{PNP}$ analogue 35. Complex 35 was dissolved in a 2:1 mixture of acetone- $\mathrm{d}_{6} / \mathrm{D}_{2} \mathrm{O}$ and the solvent composition of each reaction adjusted so they all contained the same amounts of acetone and water. A control reaction between pyridine and the substrate was also carried out. Table 5.4 summarises the results of this experiment.

Table 5.4 Comparison between ${ }^{2-p y r} \mathrm{PNP}$ and ${ }^{\mathrm{Ph}} \mathrm{PNP}$ ligands. ${ }^{a}$

\begin{tabular}{ccccc} 
entry & catalyst & $\mathbf{R}$ & time $(\mathbf{h})$ & Yield $_{(\%)^{a}}$ \\
\hline 1 & $\mathbf{3 3}$ & 2-pyridyl & 6 & 99 \\
2 & $\mathbf{3 5}$ & & 6 & 66 \\
2 & pyridine & & 6 & 11 \\
4 & - & & 6 & 18 \\
\hline 5 & $\mathbf{3 3}$ & $\mathrm{CH}_{2} \mathrm{CH}_{3}$ & 6 & 26 \\
6 & $\mathbf{3 5}$ & & 6 & 9.8 \\
7 & pyridine & & 6 & 0 \\
8 & - & & 6 & 0 \\
\hline 9 & $\mathbf{3 3}$ & $\mathrm{CH}_{3}$ & 6 & 31 \\
10 & $\mathbf{3 5}$ & & 6 & 14 \\
11 & pyridine & & 6 & 0 \\
12 & - & & 6 & 0 \\
\hline
\end{tabular}

${ }^{a} 6$ hours, $100{ }^{\circ} \mathrm{C}, 5 \mathrm{~mol} \%$ catalyst, $0.45 \mathrm{~mL} \mathrm{D} \mathrm{D}_{2} \mathrm{O} / 0.055 \mathrm{~mL}$ acetone- $\mathrm{d}_{6}$.

${ }^{a}$ Determined by NMR

In each case, employing the pyridylphosphine complex resulted in a higher yield of the amide product. This suggests that activation of water by the pyridyl nitrogens is indeed occurring. However, overall catalyst activities are low compared to many of the previously reported nitrile hydration catalysts. These often show much greater 
conversion at lower temperatures and loadings. For example, the naphthyridinecontaining rhodium complex discussed in chapter one (Figure 1.7) is able to hydrate propionitrile at $25^{\circ} \mathrm{C}$ with 10 times less catalyst (0.5 mol\%, 6 hours, $80 \%$ GC yield). ${ }^{26}$

Only three other iridium complexes have been reported to catalyse nitrile hydration. The earliest report was trans-[Ir $\left.(\mathrm{OH})(\mathrm{CO})\left(\mathrm{PPh}_{3}\right)_{2}\right]$. Otsuka et al. found that this gave a $2.6 \%$ yield of acetamide when heated in water acetonitrile for 20 hours at $140{ }^{\circ} \mathrm{C} .{ }^{166}$ The iridium complexes investigated in this work all gave better yields than this catalyst.

Chin et al. employed an iridium complex bearing water-soluble phosphine ligands, $\left[\mathrm{IrH}(\mathrm{CO})(\mathrm{TPPTS})_{3}\right] . *$ This gave a $38 \%$ yield of acetamide after 4 hours of heating at $100{ }^{\circ} \mathrm{C}(1.7 \mathrm{~mol} \%$ in water $) .{ }^{167}$ In comparison, a $31 \%$ yield was observed by NMR after 6 hours using $5 \mathrm{~mol} \%$ of the ${ }^{2-p y r} \mathrm{PNP}$ catalyst (entry 9, Table 5.4).

The most recent report is by the Marder group. ${ }^{168}$ The iridium peroxo complex cis$\left[\operatorname{Ir}\left(\mathrm{O}_{2}\right)\left(\mathrm{PMe}_{3}\right)_{4}\right] \mathrm{Cl}$ was used to hydrate acetonitrile in water. Although the catalyst loading was much lower than the $5 \mathrm{~mol} \%$ employed in the acetonitrile hydration reactions described here, the much higher temperature $\left(140{ }^{\circ} \mathrm{C}\right)$ and longer reaction time $(200 \mathrm{~h})$ mean that the activities of iridium complexes described here cannot be compared. However, one could hypothesise that the PNP complexes in this work would also reach that conversion given the extended time period and high temperature.

\subsection{Concluding Remarks}

The hydration of unsaturated substrates was investigated with four iridium PNP pincer complexes. NMR analysis of the reactions of $\mathbf{3 3}$ with alkenes and alkynes was limited with respect to determining the products of the reactions; however, in at least one case, a $\mathrm{C}-\mathrm{C}$ bond was formed. Head-to-head dimerisation of (triisopropylsilyl)acetylene occurred in the presence of $5 \mathrm{~mol} \% 33$ and formed $(E)$ 1,4-bis(triisopropylsilyl)-1-buten-3-yne (38). This is the first synthesis of $\mathbf{3 8}$ in an aqueous reaction medium.

Nitrile hydration reactions were followed by ${ }^{1} \mathrm{H}$ NMR spectroscopy. All three ${ }^{2-p y r} \mathrm{PNP}$ iridium pincer complexes are able to catalyse this reaction, albeit with lower activity than previously reported ruthenium and rhodium hydration catalysts. Iridium is

\footnotetext{
${ }^{*}$ TPPTS $=\mathrm{P}\left(\text { meta }-\mathrm{C}_{6} \mathrm{H}_{4} \mathrm{SO}_{3} \mathrm{Na}\right)_{3} \cdot \mathrm{xH}_{2} \mathrm{O}$
} 
rarely used to hydrate nitriles, and the activities of the complexes investigated in this work are likely to be comparable to those in the literature.

The bis(ethene) complex $\mathbf{3 6}$ is more active than cod complex 33 and more active than bis(hydride) complex 37 . Compound 33 was compared with its pyridyl-free analogue 35, and the pyridyl substituents were found to result in a higher yield of the amide.

\subsection{Future Work}

Investigation of the coordination chemistry of ${ }^{2-p y r} \mathrm{PNP}$ with ruthenium precursors should be carried out, as the use of a more suitable metal has a good chance of improving the catalytic activity of ${ }^{2-p y r}$ PNP complexes. Ruthenium is the most wellstudied late transition metal for nitrile hydration, and many ruthenium complexes show high activity. ${ }^{23}$ For example, the Oshiki group have reported a ruthenium $\mathrm{Ph}_{2} \mathrm{P}(2$-pyr $)$ complex that shows the highest-reported turnover frequency for the hydration of acetonitrile. ${ }^{70}$

Reactions of $\mathbf{3 3}$ with alkene and alkyne substrates should be repeated and the products investigated by GC-MS. Determining a yield for the reaction is particularly important in the case of (triisopropylsilyl)acetylene dimerisation, as the report by the Goldman group of an iridium ${ }^{t}$ Bu $\mathrm{PCP}$ complex is not catalytic. ${ }^{165}$

Due to time constraints, stoichiometric experiments to determine the process by which alkyne dimerisation occurs were not carried out. It would be interesting to determine whether it goes by the same mechanism as the iridium ${ }^{t} \mathrm{Bu} P \mathrm{CP}$ complex reaction or if the pyridyl groups or PNP backbone are involved. The nitrile hydration reactions also need to be carried out for a longer period of time to confirm that the product amides are not converted to carboxylic acids - this would result in undesirable product mixtures. 


\section{Chapter 6}

\section{Conclusion}

This study has aimed to investigate the coordination chemistry of a novel PNP pincer ligand bearing 2-pyridylphosphine substituents ( $\left.{ }^{2-p y r} \mathrm{PNP}\right)$, with the end goal being a metal complex that catalyses the hydration of unsaturated substrates in water. Such a reaction is very desirable as it forms useful products and employs an environmentally friendly solvent. A synthetic route to ${ }^{2-p y r}$ PNP has been developed, and the coordination chemistry of this ligand investigated with rhodium and iridium precursors. The iridium complexes have been evaluated as catalysts in the hydration of simple unsaturated substrates.

The first goal of this project was to synthesise ${ }^{2-p y r} \mathrm{PNP}$. Literature methods to pyridylphosphines generate intractable impurities when applied to the synthesis of ${ }^{2-p y r} \mathrm{PNP}$, so the synthesis of pure ${ }^{2-p y r} \mathrm{PNP}$ was investigated (through a sometimes frustrating process). Satisfyingly, the sources of the impurities were identified. By using high sodium lithium powder to generate $\operatorname{LiP}(2 \text {-pyr })_{2}$ and replacing the tertbutyl chloride used to quench the 2-pyridyl anions with ammonium chloride, pure ${ }^{2-p y r}$ PNP could be synthesised in high yields. This method represents an improved synthetic route to pyridylphosphines and may be applicable to the syntheses of other aryl- or heteroaryl-substituted phosphines.

The ${ }^{2-p y r}$ PNP ligand was then reacted with rhodium(I) and iridium(I) precursors. Results of the rhodium study show that ${ }^{2-p y r}$ PNP reacts very differently with rhodium to known PNP ligands and suggest that a pathway to rhodium(II) is accessible with ${ }^{2-p y r}$ PNP. This is particularly interesting as the redox activity of PNP ligands is not well-understood. However, this study is limited by the difficulty in characterising any of the rhodium ${ }^{2-p y r} \mathrm{PNP}$ complexes. When ${ }^{2-p y r} \mathrm{PNP}$ was reacted with $[\operatorname{Ir}(\operatorname{cod})(\mu-$ $\mathrm{Cl})]_{2}$, a rare example of a five-coordinate complex bearing a facially-coordinated 
pincer ligand was formed. On reaction with 5 atm ethene, bis(ethene) complex $\left[\left({ }^{2-\text { pyr }} \mathrm{PNP}\right) \operatorname{Ir}\left(\mathrm{C}_{2} \mathrm{H}_{4}\right)_{2}\right] \mathrm{Cl}$ (36) formed. In 36, the pincer ligand had rearranged to take up a meridional position, demonstrating that ${ }^{2-p y r} \mathrm{PNP}$ is flexible with regards to its coordination geometry. Such flexibility can be beneficial for a catalyst as it places fewer constraints on the geometries available throughout the catalytic cycle. Hydrogenation of $\mathbf{3 3}$ gave chloroiridium(III) bis(hydride) complex $\left[\left({ }^{2-p y r} \mathrm{PNP}\right) \operatorname{Ir}(\mathrm{H})_{2} \mathrm{Cl}\right]$ $(37)$.

Complex 33 was tested in the hydration of alkenes and alkynes in 1:1 $\mathrm{D}_{2} \mathrm{O} /$ acetone- $_{6}$. The products of these reactions could only be analysed by NMR. However, in one case, the product was easily identified as the head-to-head dimer of (triisopropylsilyl)acetylene, enyne 38. Dimerisation of terminal alkynes facilitated by transition metal complexes is a common reaction, but this is the first time that $\mathbf{3 8}$ has been synthesised under aqueous conditions.

Complexes 33, 36, and $\mathbf{3 7}$ displayed fair activity towards the hydration of nitriles in water. This is the first example of hydration carried out by a pincer complex, and is one of three examples of iridium-catalysed nitrile hydration. Gratifyingly, the complex containing pyridyl groups was more active in the hydration of nitriles than its phenyl-substituted analogue. As a result of this success, an investigation into the coordination chemistry of ${ }^{2-p y r} \mathrm{PNP}$ would be warranted, as ruthenium complexes show more promise as nitrile hydration catalysts than iridium complexes.

Over the years, the pincer framework has been shown by many researchers to be extremely versatile. Development of a new pincer ligand in this work has not only shown that literature routes to pyridylphosphines have problems, and solved them: it has also shown that the ligand is able to facilitate improved yields of nitrile hydration reactions compared to a pyridyl-free analogue. 


\section{Chapter 7}

\section{Experimental}

\subsection{General Procedures}

Reactions and manipulations of products and reagents were carried out under an inert argon atmosphere using standard Schlenk techniques, unless otherwise stated. Diethyl ether and tetrahydrofuran were distilled under an argon atmosphere from sodium/benzophenone ketyl prior to use. Other solvents were degassed prior to use, and were stored over molecular sieves if appropriate. $\mathrm{Vi}_{2} \mathrm{P}\left(\mathrm{NEt}_{2}\right),{ }^{169} \mathrm{P}(2 \text {-pyr })_{3},{ }^{170} 2,6$ bis(chloromethyl)pyridine, ${ }^{82}$ 2,6-bis(bromomethyl)pyridine, ${ }^{81}\left[\mathrm{Mg}(\text { anth })(\mathrm{THF})_{3}\right]^{171}$ and the metal precursors $\left[\operatorname{Ir}(\operatorname{cod})_{2}\right] \mathrm{BF}_{4},{ }^{172}[\operatorname{Ir}(\operatorname{cod})(\mu-\mathrm{Cl})]_{2},{ }^{173}[\operatorname{Ir}(\operatorname{cod})(\mu-\mathrm{OMe})]_{2},{ }^{174}$ $\left[\mathrm{Rh}(\mathrm{coe})_{2}(\mu-\mathrm{Cl})\right]_{2},{ }^{175}$ were synthesised according to literature procedures. All other reagents were obtained from commercial suppliers and used without further purification.

NMR spectra were recorded on a Varian Inova 300 spectrometer $\left(300 \mathrm{MHz}\right.$ for ${ }^{1} \mathrm{H}$, $282 \mathrm{MHz}$ for ${ }^{19} \mathrm{~F}, 121 \mathrm{MHz}$ for ${ }^{31} \mathrm{P}$, and $75 \mathrm{MHz}$ for ${ }^{13} \mathrm{C}$ ); a Varian Unity Inova 500 (500 MHz for ${ }^{1} \mathrm{H}, 160 \mathrm{MHz}$ for ${ }^{11} \mathrm{~B}, 125 \mathrm{MHz}$ for ${ }^{13} \mathrm{C}$, and $121 \mathrm{MHz}$ for ${ }^{31} \mathrm{P}$ ); and a Varian DirectDrive 600 (600 MHz for ${ }^{1} \mathrm{H}, 150 \mathrm{MHz}$ for ${ }^{13} \mathrm{C}, 60 \mathrm{MHz}$ for $\left.{ }^{15} \mathrm{~N}\right) .{ }^{31} \mathrm{P},{ }^{13} \mathrm{C}$ and ${ }^{19} \mathrm{~F}$ spectra were proton-decoupled unless specified. Chemical shifts are reported in ppm relative to the residual solvent peak; ${ }^{176}{ }^{31} \mathrm{P},{ }^{19} \mathrm{~F},{ }^{15} \mathrm{~N}$, and ${ }^{11} \mathrm{~B}$ resonances are referenced to $\mathrm{H}_{3} \mathrm{PO}_{4}, \mathrm{CFCl}_{3}, \mathrm{CH}_{3} \mathrm{NO}_{2}$, and $\mathrm{BF}_{3}$. OEt 2 respectively. Splitting patterns are abbreviated as: s: singlet, $\mathrm{d}$ : doublet, $\mathrm{t}$ : triplet, vt: virtual triplet, $\mathrm{m}$ : multiplet, dm: doublet of multiplets, br: broad.

Infrared spectra were obtained with a PerkinElmer Spectrum One FT-IR spectrometer as KBr discs, or with a Bruker ALPHA Platinum FT-IR spectrometer. 
Electrospray ionisation mass spectroscopy was recorded using an Agilent 6530 Q-TOF mass spectrometer. Elemental analysis was performed at the Campbell Microanalytical Laboratory at the University of Otago, Dunedin.

X-ray diffraction data were collected at the University of Canterbury on an Agilent SuperNova CCD diffractometer. Data were reduced using Agilent CrysAlisPro software. Absorption correction was performed using the SCALE3 ABSPACK programs. OLEX2 (Version 1.2.6) ${ }^{177}$ was used as a front-end for SHELX or Superflip ${ }^{178-180}$ executables during structure solution and refinement. The positions of all hydrogen atoms were calculated during refinement.

\subsection{Ligand Synthesis}

\section{$1,3-\left[\left({ }^{t} \mathrm{Bu}\right)_{2} \mathrm{P}\left(\mathrm{BH}_{3}\right) \mathrm{CH}_{2}\right]_{2} \mathrm{C}_{5} \mathrm{H}_{3} \mathrm{~N}\left({ }^{t} \mathrm{Bu} \mathrm{PNP}\left(\mathrm{BH}_{3}\right), 8\right)$}

${ }^{t} \mathrm{Bu}_{2} \mathrm{PH}\left(\mathrm{BH}_{3}\right)$ (0.290 g, $\left.1.81 \mathrm{mmol}\right)$ was dissolved in THF $(15 \mathrm{~mL})$ and the solution cooled to $0{ }^{\circ} \mathrm{C}$. $n$-BuLi $(1.00 \mathrm{~mL}, 2.00 \mathrm{mmol})$ was added slowly with stirring. The cooling bath was removed and the solution allowed to warm to room temperature over 30 minutes. After cooling the reaction mixture to $-78{ }^{\circ} \mathrm{C}$, a solution of 2,6 bis(chloromethyl)pyridine $(0.160 \mathrm{~g}, 0.909 \mathrm{mmol})$ in THF $(2 \mathrm{~mL})$ was added dropwise. The resulting pale yellow solution was stirred for 2 hours. The solution was washed with water $(2 \times 20 \mathrm{~mL})$, the organic layer dried with $\mathrm{MgSO}_{4}$, and finally the solvent removed in vacuo. Purification by recrystallisation from 2:1 hexane/toluene gave 8 as small white needles (257 mg, 67\%).

${ }^{1} \mathrm{H}$ NMR: $\left(500 \mathrm{MHz}, \mathrm{CDCl}_{3}\right) \delta_{H} 7.53\left(\mathrm{t},{ }^{3} J_{\mathrm{H}-\mathrm{H}}=7.8 \mathrm{~Hz}, 1 \mathrm{H}\right.$, pyridine-4), 7.45 (d, ${ }^{3} J_{\mathrm{H}-\mathrm{H}}=7.7 \mathrm{~Hz}, 2 \mathrm{H}$, pyridine-3,5), $3.27\left(\mathrm{~d},{ }^{2} J_{\mathrm{P}-\mathrm{H}}=12.3 \mathrm{~Hz}, 4 \mathrm{H}, \mathrm{P}-\mathrm{CH}_{2}\right), 1.27$ (d, $\left.{ }^{3} J_{\mathrm{P}-\mathrm{H}}=12.6 \mathrm{~Hz}, 36 \mathrm{H}, \mathrm{CH}_{3}\right) .{ }^{13} \mathrm{C}\left\{{ }^{1} \mathrm{H}\right\}$ NMR: (125 MHz, $\left.\mathrm{CDCl}_{3}\right) \delta_{C} 154.6$ (s, pyridine2,6), 136.1 (s, pyridine-4), 123.9 (s, pyridine-3,5), 32.9 (d, ${ }^{1} J_{\mathrm{P}-\mathrm{C}}=23.7 \mathrm{~Hz}, C_{C H}$ ), $29.0\left(\mathrm{~d},{ }^{1} J_{\mathrm{P}-\mathrm{C}}=22.8 \mathrm{~Hz}, \mathrm{P}-\mathrm{CH}_{2}\right), 28.3\left(\mathrm{~s}, \mathrm{CH}_{3}\right) .{ }^{31} \mathrm{P}\left\{{ }^{1} \mathrm{H}\right\} \mathrm{NMR}:\left(202 \mathrm{MHz}, \mathrm{CDCl}_{3}\right)$ $\delta_{P} 46.91$ (br m). ${ }^{11} \mathrm{~B}\left\{{ }^{1} \mathrm{H}\right\}$ NMR: (160 MHz, $\left.\mathrm{CDCl}_{3}\right) \delta_{B}-42.29$ (br m). HRMS calcd for $\mathrm{C}_{23} \mathrm{H}_{50} \mathrm{~B}_{2} \mathrm{NP}_{2}[\mathrm{M}+\mathrm{H}]^{+}: \mathrm{m} / z=424.3607$; found: 421.3599. Anal. calcd for $\mathrm{C}_{23} \mathrm{H}_{49} \mathrm{~B}_{2} \mathrm{NP}_{2}$ : C, 65.12; H, 11.88 ; N, 3.30; found: C, 65.24; H, 11.74; N, 3.14. IR $(\mathrm{KBr}): \nu_{\max } / \mathrm{cm}^{-1}=2408,2369,2338(\mathrm{~B}-\mathrm{H}$ stretch$) ; 1587,1579$ (backbone $\mathrm{C}=\mathrm{N}$ stretch); 1450 (backbone $\mathrm{C}=\mathrm{C}$ stretch). 


\section{$1,3-\left[\left({ }^{t} \mathrm{Bu}\right)_{2} \mathrm{PCH}_{2}\right]_{2} \mathrm{C}_{5} \mathrm{H}_{3} \mathrm{~N}\left({ }^{t}{ }^{\mathrm{Bu}} \mathrm{PNP}, 6\right)$}

8 (20.0 mg, $0.0472 \mathrm{mmol})$ was heated with morpholine $(0.5 \mathrm{~mL})$ at $100{ }^{\circ} \mathrm{C}$ for 1 hour. The solvent was removed under reduced pressure, and the white residue extracted with $n$-hexane $(2 \times 0.5 \mathrm{~mL})$, and filtered through a plug of alumina. Removal of the solvent gave 6 as a white solid (16.0 mg, 86\%). NMR spectra agreed with reported data. $^{79}$

${ }^{31} \mathrm{P}\left\{{ }^{1} \mathrm{H}\right\}$ NMR: (121 MHz, $\left.\mathrm{C}_{6} \mathrm{D}_{6}\right) \delta_{P} 35.04(\mathrm{~s})$.

\section{1,3-[(2-pyr $\left.)_{2} \mathrm{PCH}_{2}\right]_{2} \mathrm{C}_{5} \mathrm{H}_{3} \mathrm{~N}\left({ }^{2-p y r} \mathrm{PNP}\right)$ via $\mathrm{P}(2-\mathrm{pyr})_{3}$ and potassium}

To a solution of $\mathrm{P}(2 \text {-pyr })_{3}(0.716 \mathrm{~g}, 2.70 \mathrm{mmol})$ in THF $(10 \mathrm{~mL})$ was added small chunks of potassium $(0.296 \mathrm{~g}, 7.57 \mathrm{mmol})$. This was stirred vigorously for 16 hours, resulting in a very dark solution. The reaction mixture was cooled to $-78^{\circ} \mathrm{C}$, then tert-butyl chloride added, resulting in a colour change to dark red. The cooling bath was removed and the solution stirred for a further 30 minutes before being cooled to $-78{ }^{\circ} \mathrm{C}$. A solution of 2,6-bis(bromomethyl)pyridine in THF (2 mL) was added, and the resulting dark purple solution stirred for 40 minutes. THF was removed under vacuum, followed by the addition of dichloromethane $(20 \mathrm{~mL})$ and degassed distilled water $(20 \mathrm{~mL})$. The cloudy orange solution was stirred for 30 minutes, then the layers allowed to separate. The organic layer was dried under reduced pressure to yield a brown oil (56\%).

${ }^{31} \mathrm{P}\left\{{ }^{1} \mathrm{H}\right\}$ NMR: $\left(202 \mathrm{MHz}, \mathrm{CDCl}_{3}\right) \delta_{P}-6.48(\mathrm{~s})$.

NMR data showed that the desired product was present but very impure. Recrystallisation was attempted from $n$-propanol/ethanol, $n$-propanol/hexane, and pyridine $/ n$-pentane, but did not yield pure product.

\section{1,3-[(2-pyr $\left.)_{2} \mathrm{PCH}_{2}\right]_{2} \mathrm{C}_{5} \mathrm{H}_{3} \mathrm{~N}\left({ }^{2-p y r} \mathrm{PNP}\right)$ via $\mathrm{P}(2-\mathrm{pyr})_{3}$ and lithium powder}

Lithium dispersion (high sodium, dispersed in paraffin oil, $80 \mathrm{mg}$ ) was washed with $n$-hexane $(3 \times 2 \mathrm{~mL})$, then the $n$-hexane was removed and the lithium re-weighed 
(68.8 $\mathrm{mg}, 9.91 \mathrm{mmol})$. The lithium powder was suspended in THF ( $5 \mathrm{~mL}$ ) and cooled to $-78{ }^{\circ} \mathrm{C}$ then $\mathrm{P}(2 \text {-pyr })_{3}(0.904 \mathrm{~g}, 3.41 \mathrm{mmol})$ was added as a solid. The reaction mixture was stirred for 60 minutes, after which time it was dark red. Unreacted lithium was filtered off, then the solution cooled to $-78{ }^{\circ} \mathrm{C} . \mathrm{NH}_{4} \mathrm{Cl}(0.170 \mathrm{~g}, 3.18$ mmol) was added, then the cooling bath removed and the reaction mixture stirred for 30 minutes. Solid 2,6-bis(bromomethyl)pyridine (0.307 g, $1.16 \mathrm{mmol})$ was added in portions, resulting in immediate lightening of the solution. After 1 hour the THF was removed leaving a bubbly orange residue. Dichloromethane $(5 \mathrm{~mL})$ and water $(5$ $\mathrm{mL}$ ) were added, the water layer removed, then the yellow organic layer washed with saturated $\mathrm{NaHCO}_{3}$. The dichloromethane was evaporated under reduced pressure and the 2,2-bipyridine impurity removed by sublimation to leave the title compound as a yellow-orange oil (78\%).

${ }^{1} \mathrm{H}$ NMR: (500 MHz, $\left.\mathrm{CDCl}_{3}\right) \delta_{H} 8.66(\mathrm{~m}, 4 \mathrm{H}$, pyr-3), 7.54 (m, 4H, pyr-5), 7.51 $\left(\mathrm{m}, 4 \mathrm{H}\right.$, pyr-6), $7.25\left(\mathrm{t},{ }^{3} J_{\mathrm{H}-\mathrm{H}}=8.1 \mathrm{~Hz}, 1 \mathrm{H}\right.$, pyridine- 4$), 7.13(\mathrm{~m}, 4 \mathrm{H}$, pyr-4), 6.90 $\left(\mathrm{d},{ }^{3} J_{\mathrm{H}-\mathrm{H}}=8.0 \mathrm{~Hz}, 2 \mathrm{H}\right.$, pyridine-3,5), $3.95\left(\mathrm{~s}, 4 \mathrm{H}, \mathrm{P}-\mathrm{CH}_{2}\right),{ }^{13} \mathrm{C}\left\{{ }^{1} \mathrm{H}\right\}$ NMR: $(125$ $\left.\mathrm{MHz}, \mathrm{CDCl}_{3}\right) \delta_{C} 163.0\left(\mathrm{~d},{ }^{2} J_{\mathrm{P}-\mathrm{C}}=4.9 \mathrm{~Hz}\right.$, pyridine-2,6), $157.8\left(\mathrm{~d},{ }^{3} J_{\mathrm{P}-\mathrm{C}}=8.6 \mathrm{~Hz}\right.$, pyr-1), 150.1 (d, ${ }^{1} J_{\mathrm{P}-\mathrm{C}}=8.6 \mathrm{~Hz}$, pyr-3), 136.3 (s, pyridine-4), 135.5 (d, ${ }^{2} J_{\mathrm{P}-\mathrm{C}}=5.4 \mathrm{~Hz}$, pyr-5), 129.0 (d, ${ }^{2} J_{\mathrm{P}-\mathrm{C}}=25.6 \mathrm{~Hz}$, pyr-6), 122.5 (s, pyr-4), 120.9 (d, ${ }^{3} J_{\mathrm{P}-\mathrm{C}}=5.9 \mathrm{~Hz}$, pyridine-3,5), $35.8\left(\mathrm{~d},{ }^{1} J_{\mathrm{P}-\mathrm{C}}=13.8 \mathrm{~Hz}, \mathrm{P}-\mathrm{CH}_{2}\right) .{ }^{31} \mathrm{P}\left\{{ }^{1} \mathrm{H}\right\} \mathrm{NMR}:\left(202 \mathrm{MHz}, \mathrm{CDCl}_{3}\right)$ $\delta_{P}-6.48(\mathrm{~s}) .{ }^{15} \mathrm{~N}$ NMR: (60 MHz, $\left.\mathrm{CDCl}_{3}\right) \delta_{N}-57.4$ (pyr), -66.1 (pyridine).

Infrared spectroscopy and mass spectrometry were carried out on a sample of ${ }^{2-p y r} P N P$ oxide, as ${ }^{2-p y r}$ PNP is extremely air-sensitive.

\section{$1,3-\left[\mathrm{Ph}_{2} \mathrm{PCH}_{2}\right]_{2} \mathrm{C}_{5} \mathrm{H}_{3} \mathrm{~N}\left({ }^{\mathrm{Ph}} \mathrm{PNP}\right)$ via $\mathrm{P}(2-\mathrm{pyr})_{3}$ and lithium powder}

Lithium dispersion (high sodium, dispersed in paraffin oil) was washed with $n$-hexane $(3 \times 2 \mathrm{~mL})$, then the $n$-hexane was removed and the lithium re-weighed $(35.0 \mathrm{mg}, 5.04$ $\mathrm{mmol})$. The lithium powder was suspended in THF $(4 \mathrm{~mL})$ and cooled to $-78{ }^{\circ} \mathrm{C}$ then $\mathrm{PPh}_{3}(0.438 \mathrm{~g}, 1.67 \mathrm{mmol})$ was added as a solid. The reaction mixture was stirred for 60 minutes, after which time it was dark red. Unreacted lithium was filtered off, then the solution cooled to $-78{ }^{\circ} \mathrm{C} . \mathrm{NH}_{4} \mathrm{Cl}(89.0 \mathrm{mg}, 1.66 \mathrm{mmol})$ was added, then the cooling bath removed and the reaction mixture stirred for 30 minutes. Solid 2,6-bis(chloromethyl)pyridine (123 mg, $0.699 \mathrm{mmol}$ ) was added in portions, resulting in immediate lightening of the solution. After 10 minutes the THF was removed leaving a greyish residue. Dichloromethane $(5 \mathrm{~mL})$ and water $(5 \mathrm{~mL})$ were added and the water layer removed. The dichloromethane was evaporated under reduced 
pressure and the white powdery solid washed with $n$-hexane (282 $\mathrm{mg}, 85 \%)$.

${ }^{1} \mathrm{H}$ NMR: $\left(500 \mathrm{MHz}, \mathrm{CDCl}_{3}\right) \delta_{H} 8.66(\mathrm{~m}, 4 \mathrm{H}, \mathrm{pyr}-3), 7.54(\mathrm{~m}, 4 \mathrm{H}, \mathrm{pyr}-5), 7.51(\mathrm{~m}, 4 \mathrm{H}$, pyr-6), 7.25 (t, ${ }^{3} J_{\mathrm{H}-\mathrm{H}}=8.1 \mathrm{~Hz}, 1 \mathrm{H}$, pyridine-4), $7.13\left(\mathrm{~m}, 4 \mathrm{H}\right.$, pyr-4), 6.90 (d, ${ }^{3} J_{\mathrm{H}-\mathrm{H}}=8$ $\mathrm{Hz}, 2 \mathrm{H}$, pyridine-3,5), $3.95\left(\mathrm{~s}, 4 \mathrm{H}, \mathrm{P}-\mathrm{CH}_{2}\right),{ }^{13} \mathrm{C}\left\{{ }^{1} \mathrm{H}\right\} \mathrm{NMR}:\left(125 \mathrm{MHz}, \mathrm{CDCl}_{3}\right) \delta_{C}$ $163.0\left(\mathrm{~d},{ }^{2} J_{\mathrm{P}-\mathrm{C}}=4.9 \mathrm{~Hz}\right.$, pyridine-2,6), 157.8 (d, ${ }^{3} J_{\mathrm{P}-\mathrm{C}}=8.6 \mathrm{~Hz}$, pyr-1), 150.1 (d, ${ }^{1} J_{\mathrm{P}-\mathrm{C}}=8.6 \mathrm{~Hz}$, pyr-3), 136.3 (s, pyridine-4), 135.5 (d, ${ }^{2} J_{\mathrm{P}-\mathrm{C}}=5.4 \mathrm{~Hz}$, pyr-5), 129.0 (d,

${ }^{2} J_{\mathrm{P}-\mathrm{C}}=25.6 \mathrm{~Hz}$, pyr-6), 122.5 (s, pyr-4), $120.9\left(\mathrm{~d},{ }^{3} J_{\mathrm{P}-\mathrm{C}}=5.9 \mathrm{~Hz}\right.$, pyridine-3,5), 35.8 $\left(\mathrm{d},{ }^{1} J_{\mathrm{P}-\mathrm{C}}=13.8 \mathrm{~Hz}, \mathrm{P}-\mathrm{CH}_{2}\right) .{ }^{31} \mathrm{P}\left\{{ }^{1} \mathrm{H}\right\}$ NMR: (202 $\left.\mathrm{MHz} \mathrm{CDCl}_{3}\right) \delta_{P}-10.96(\mathrm{~s})$.

\section{$(2-p y r){ }_{2} \mathrm{P}(\mathrm{O}) \mathrm{H}(24)$}

This reaction produces strong-smelling products. All manipulations were carried out in a fume hood on "boost"; all glassware, needles, and syringes were rinsed copiously with a bleach solution after use.

A two-necked flask fitted with a dropping funnel was charged with isopropylmagnesium chloride $(10 \mathrm{~mL}, 20.0 \mathrm{mmol})$, and the solution cooled to $0{ }^{\circ} \mathrm{C}$. A solution of 2-bromopyridine $(1.91 \mathrm{~mL}, 20.0 \mathrm{mmol})$ in THF $(10 \mathrm{~mL})$ was added dropwise. The cooling bath was removed following addition, and the dark brown solution stirred for three hours. The reaction mixture (which may be clear, or contain a brown precipitate) was then cooled to $0{ }^{\circ} \mathrm{C}$ and diethyl phosphite $(0.810 \mathrm{~mL}, 6.25 \mathrm{mmol})$ added dropwise. The reaction mixture was stirred overnight, then degassed water added $(0.3 \mathrm{~mL})$. The solvent was removed under reduced pressure, resulting in a pale orange residue. Chloroform $(30 \mathrm{~mL})$ and water $(20 \mathrm{~mL})$ were added, and the mixture transferred to a separating funnel. The organic layer was dried over $\mathrm{MgSO}_{4}$, then dried under reduced pressure to give $(2-\mathrm{pyr}){ }_{2} \mathrm{P}(\mathrm{O}) \mathrm{H}$ as a tan powder $(1.04 \mathrm{~g}, 82 \%)$.

${ }^{1} \mathrm{H}$ NMR: $\left(500 \mathrm{MHz}, \mathrm{CDCl}_{3}\right) \delta_{H} 8.77\left(\mathrm{~m}, 2 \mathrm{H}\right.$, pyr-3), $8.07\left(\mathrm{~m},{ }^{1} J_{\mathrm{PH}}=511.0 \mathrm{~Hz}, 1 \mathrm{H}\right.$, $\mathrm{PH}), 8.04$ (m, 2H, pyr-6), 7.85 ( $\mathrm{m}, 2 \mathrm{H}$, pyr-5), 7.43 (m, 2H, pyr-4). ${ }^{13} \mathrm{C}\left\{{ }^{1} \mathrm{H}\right\} \mathrm{NMR}$ : $\left(125 \mathrm{MHz}, \mathrm{CDCl}_{3}\right) \delta_{C} 153.9\left(\mathrm{~d},{ }^{1} J_{\mathrm{P}-\mathrm{C}}=130.0 \mathrm{~Hz}, \mathrm{pyr}-1\right), 150.7\left(\mathrm{~d},{ }^{3} J_{\mathrm{P}-\mathrm{C}}=22.0 \mathrm{~Hz}\right.$, pyr-3), 136.5 (d, ${ }^{3} J_{\mathrm{P}-\mathrm{C}}=10.0 \mathrm{~Hz}$, pyr-5), $127.6\left(\mathrm{~d},{ }^{2} J_{\mathrm{P}-\mathrm{C}}=22.7 \mathrm{~Hz}\right.$, pyr-6), 126.2 $\left(\mathrm{d},{ }^{4} J_{\mathrm{P}-\mathrm{C}}=4.0 \mathrm{~Hz}\right.$, pyr-4). ${ }^{31} \mathrm{P}\left\{{ }^{1} \mathrm{H}\right\} \mathrm{NMR}:\left(202 \mathrm{MHz}, \mathrm{CDCl}_{3}\right) \delta_{P} 17.2(\mathrm{~s})$. HRMS calcd for $\mathrm{C}_{10} \mathrm{H}_{10} \mathrm{~N}_{2} \mathrm{OP}[\mathrm{M}+\mathrm{H}]^{+}: m / z=205.0525$; found: 205.0529. Anal. calcd for $\mathrm{C}_{10} \mathrm{H}_{9} \mathrm{~N}_{2} \mathrm{OP}$ : C, 58.83; H, 4.44; N, 13.72; found: C, 57.26; H, 4.56; N, 13.10 . Compound $\mathbf{2 4}$ is hygroscopic. The sample was dried under vacuum for 24 hours but this was not sufficient. Heating causes decomposition to (2-pyr) ${ }_{2} \mathrm{PH}$. IR (KBr): $\nu_{\max } / \mathrm{cm}^{-1}=2333(\mathrm{P}-\mathrm{H}$ stretch $), 1575(\mathrm{C}=\mathrm{N}$ stretch $), 1193(\mathrm{P}=\mathrm{O}$ stretch$)$. 


\section{1,3-[(2-pyr $\left.)_{2} \mathrm{P}(\mathrm{O}) \mathrm{CH}_{2}\right]_{2} \mathrm{C}_{5} \mathrm{H}_{3} \mathrm{~N}\left({ }^{2-p y r} \mathrm{PNP}-\mathrm{O}_{2}, 25\right)$}

A $60 \%$ dispersion of sodium hydride in mineral oil $(53.0 \mathrm{mg}, 2.20 \mathrm{mmol})$ was suspended in THF $(5 \mathrm{~mL})$. Solid (2-pyr $)_{2} \mathrm{P}(\mathrm{O}) \mathrm{H}(0.301 \mathrm{~g}, 1.47 \mathrm{mmol})$ was added in small portions. The resulting orange solution was stirred at room temperature for 10 minutes, then 2,6-bis(bromomethyl)pyridine added (0.195 mg, $0.736 \mathrm{mmol}$ ). This resulted in the immediate formation of a white solid. After addition of water $(0.1$ $\mathrm{mL})$, the solid was collected on a frit, washed with petroleum ether $(3 \mathrm{~mL})$, then dried under reduced pressure. (252 $\mathrm{mg}, 67 \% \mathbf{2 5}$ ).

${ }^{1} \mathrm{H}$ NMR: (500 MHz, D $\left.2 \mathrm{O}\right) \delta_{H} 8.68$ (m, 4H, pyr-3), 7.98 (m, 4H, pyr-5), 7.85 (n. $4 \mathrm{H}$, pyr-6), $7.62\left(\mathrm{~m}, 4 \mathrm{H}\right.$, pyr-4), $7.47\left(\mathrm{t},{ }^{3} J_{\mathrm{H}-\mathrm{H}}=7.8 \mathrm{~Hz}, 1 \mathrm{H}\right.$, pyridine-4), 6.97 (d, ${ }^{3} J_{\mathrm{H}-\mathrm{H}}=7.8 \mathrm{~Hz}, 2 \mathrm{H}$. pyridine-3,5), $4.05\left(\mathrm{~d},{ }^{2} J_{\mathrm{P}-\mathrm{H}}=14.8 \mathrm{~Hz}, 4 \mathrm{H}, \mathrm{P}-\mathrm{CH}_{2}\right) .{ }^{13} \mathrm{C}\left\{{ }^{1} \mathrm{H}\right\}$ NMR: (125 MHz, D $\left.\mathrm{D}_{2} \mathrm{O}\right) \delta_{C} 151.4\left(\mathrm{~d},{ }^{1} J_{\mathrm{P}-\mathrm{C}}=132.0 \mathrm{~Hz}, \mathrm{pyr}-1\right), 150.9\left(\mathrm{~d},{ }^{2} J_{\mathrm{P}-\mathrm{C}}=9.4\right.$ $\mathrm{Hz}$, pyridine-2,6), 150.5 (d, ${ }^{3} J_{\mathrm{P}-\mathrm{C}}=19.1 \mathrm{~Hz}$, pyr-3), 138.1 (s, pyridine-4), 137.7 (d, ${ }^{3} J_{\mathrm{P}-\mathrm{C}}=9.5 \mathrm{~Hz}$, pyr-5), $128.5\left(\mathrm{~d},{ }^{6} J_{\mathrm{P}-\mathrm{C}}=20.4 \mathrm{~Hz}\right.$, pyr-6$), 127.3\left(\mathrm{~d},{ }^{4} J_{\mathrm{P}-\mathrm{C}}=3.1 \mathrm{~Hz}\right.$, pyr4), $123.6\left(\mathrm{~m}\right.$, pyridine-3,5), $36.1\left(\mathrm{~d},{ }^{1} J_{\mathrm{P}-\mathrm{C}}=65.5 \mathrm{~Hz}, \mathrm{P}-\mathrm{CH}_{2}\right) .{ }^{31} \mathrm{P}\left\{{ }^{1} \mathrm{H}\right\} \mathrm{NMR}:(202$ $\left.\mathrm{MHz}, \mathrm{D}_{2} \mathrm{O}\right) \delta_{P} 31.67(\mathrm{~s}) .{ }^{31} \mathrm{P}\left\{{ }^{1} \mathrm{H}\right\} \mathrm{NMR}:\left(121 \mathrm{MHz}, \mathrm{CDCl}_{3}\right) \delta_{P} 26.3(\mathrm{~s}) .{ }^{15} \mathrm{~N}$ NMR: $\left(60 \mathrm{MHz}, \mathrm{D}_{2} \mathrm{O}\right) \delta_{N}-71.4$ (pyr), -71.8 (pyridine). HRMS calcd for $\mathrm{C}_{27} \mathrm{H}_{24} \mathrm{~N}_{5} \mathrm{O}_{2} \mathrm{P}_{2}$ $[\mathrm{M}+\mathrm{H}]^{+}: m / z=512.14$; found: 512.1351. IR (KBr): $\nu_{\max } / \mathrm{cm}^{-1}=1638,1618$ (pyridyl $\mathrm{C}=\mathrm{N}$ stretch); 1589, 1573 (backbone $\mathrm{C}=\mathrm{N}$ stretch); 1453, 1424 (backbone $\mathrm{C}=\mathrm{C}$ stretch).

\section{1,3-[(2-pyr $\left.)_{2} \mathrm{PCH}_{2}\right]_{2} \mathrm{C}_{5} \mathrm{H}_{3} \mathrm{~N}\left({ }^{2-p y r} \mathrm{PNP}\right)$ via reduction of 25}

25 (0.250 g, $0.490 \mathrm{mmol})$ was dissolved in dry acetonitrile $(6 \mathrm{~mL})$. Triethylamine $(0.68 \mathrm{~mL}, 4.99 \mathrm{mmol})$ was added, followed by careful addition of trichlorosilane $(0.49$ $\mathrm{mL}, 4.85 \mathrm{mmol})$. The mixture was refluxed for 24 hours. A solution of $\mathrm{KOH}(4.7 \mathrm{~g}$ pellets $(85 \%)$ in $10 \mathrm{~mL}$ water) was added dropwise. The organic layer was removed and the aqueous layer extracted with dichloromethane $(3 \times 5 \mathrm{~mL})$. The yellow solution was dried under reduced pressure then washed with pentane. Approximately $90 \%$ pure by ${ }^{1} \mathrm{H}$ NMR, single ${ }^{31} \mathrm{P}\left\{{ }^{1} \mathrm{H}\right\}$ NMR signal at $-6.4 \mathrm{ppm}(\mathrm{s})$. NMR data of the pyridyl phosphine product match those obtained via $\mathrm{P}(2 \text {-pyr })_{3}$ and lithium powder. 


\section{2,6-bis[(diethoxyphosphoryl)methyl]pyridine (15)}

Synthesised using a modified literature procedure ${ }^{98,99}$

To 2,6-bis(bromomethyl)pyridine $(0.360 \mathrm{~g}, 1.36 \mathrm{mmol})$ under argon was added triethyl phosphite $(5.00 \mathrm{~mL}, 29.2 \mathrm{mmol})$. The mixture was heated to $140{ }^{\circ} \mathrm{C}$ with stirring for 3 hours, then the excess triethyl phosphite was removed under vacuum to give a pale yellow oil. Quantitative conversion. NMR data matched literature values.

${ }^{1} \mathrm{H}$ NMR: $\left(500 \mathrm{MHz}, \mathrm{CDCl}_{3}\right) \delta_{H} 7.55\left(\mathrm{t},{ }^{3} J_{\mathrm{H}-\mathrm{H}}=7.8 \mathrm{~Hz}, 1 \mathrm{H}\right.$, pyridine-4) $7.23(\mathrm{~d}$, ${ }^{3} J_{\mathrm{H}-\mathrm{H}}=7.5 \mathrm{~Hz}, 2 \mathrm{H}$, pyridine-3,5), $4.03\left(\mathrm{~m}, 8 \mathrm{H}, \mathrm{P}-\mathrm{CH}_{2}\right), 3.34\left(\mathrm{~d},{ }^{2} J_{\mathrm{P}-\mathrm{H}}=21.3 \mathrm{~Hz}, 4 \mathrm{H}\right.$, $\left.\mathrm{P}-\mathrm{CH}_{2}\right), 1.22\left(\mathrm{t},{ }^{3} J_{\mathrm{H}-\mathrm{H}}=7.3 \mathrm{~Hz}, \mathrm{CH}_{3}\right) .{ }^{31} \mathrm{P}\left\{{ }^{1} \mathrm{H}\right\} \mathrm{NMR}:\left(121 \mathrm{MHz}, \mathrm{CDCl}_{3}\right) \delta_{P} 25.18$ $(\mathrm{s})$.

\section{2,6-bis(phosphinomethyl)pyridine $\left({ }^{\mathrm{H}} \mathrm{PNP}\right)$}

Procedure adapted from literature synthesis of ${ }^{H} \mathrm{PCP} .{ }^{97}$

A flame-dried Schlenk tube was charged with diethyl ether $(5 \mathrm{~mL})$, which was then cooled to $-78{ }^{\circ} \mathrm{C}$ and $\mathrm{LiAlH}_{4}(0.250 \mathrm{~g}, 6.59 \mathrm{mmol})$ added. TMSCl $(0.838$ $\mathrm{mL}, 6.60 \mathrm{mmol}$ ) was added in portions over 5 minutes. The grey suspension was stirred at $-78^{\circ} \mathrm{C}$ for 15 minutes before being allowed to warm to room temperature over 1 hour. The reaction mixture was then cooled to $0{ }^{\circ} \mathrm{C}$ and a solution of 2,6 bis[(diethoxyphosphoryl)methyl]pyridine (15) (0.493 g, $1.30 \mathrm{mmol})$ in diethyl ether $(4 \mathrm{~mL})$ added in $0.5 \mathrm{~mL}$ portions. After 1 hour, the reaction mixture was cooled to $0{ }^{\circ} \mathrm{C}$. Degassed $\mathrm{H}_{2} \mathrm{O}(5 \mathrm{~mL})$ was added carefully, followed by $5 \mathrm{~mL}$ of a $30 \% \mathrm{NaOH}$ solution. The aqueous layer of the yellowish mixture was extracted with diethyl ether $(5 \times 8 \mathrm{~mL})$. The ether layer was washed with brine $(8 \mathrm{~mL})$, then dried over $\mathrm{MgSO}_{4}$. The solvent was removed to give a colourless oil, which became greenish brown over time as the product decomposed. A yield was not obtained due to the decomposition of ${ }^{\mathrm{H}} \mathrm{PNP}$ under reduced pressure.

${ }^{1} \mathrm{H}$ NMR: $\left(600 \mathrm{MHz}, \mathrm{C}_{6} \mathrm{D}_{6}\right) \delta_{H} 6.95\left(\mathrm{t},{ }^{3} J_{\mathrm{H}-\mathrm{H}}=7.7 \mathrm{~Hz}, 1 \mathrm{H}\right.$, pyridine-4), $6.44(\mathrm{~d}$, ${ }^{3} J_{\mathrm{H}-\mathrm{H}}=7.7 \mathrm{~Hz}, 2 \mathrm{H}$, pyridine-3,5), $2.97\left(\mathrm{dt},{ }^{1} J_{\mathrm{P}-\mathrm{H}}=192.0 \mathrm{~Hz},{ }^{2} J_{\mathrm{H}-\mathrm{H}}=7.6 \mathrm{~Hz}, 4 \mathrm{H}, \mathrm{PH}_{2}\right)$, $2.72\left(\mathrm{~m}, 4 \mathrm{H}, \mathrm{P}-\mathrm{CH}_{2}\right) .{ }^{13} \mathrm{C}\left\{{ }^{1} \mathrm{H}\right\}$ NMR: $\left(150 \mathrm{MHz}, \mathrm{C}_{6} \mathrm{D}_{6}\right) \delta_{C} 162.3$ (s, pyridine2,6), 137.0 (s, pyridine-4), 118.9 (s, pyridine-3,5), 23.1 (d, ${ }^{1} J_{\mathrm{P}-\mathrm{C}}=12.1 \mathrm{~Hz}, \mathrm{P}-\mathrm{CH}_{2}$ ). ${ }^{31} \mathrm{P}\left\{{ }^{1} \mathrm{H}\right\}$ NMR: $\left(121 \mathrm{MHz}, \mathrm{C}_{6} \mathrm{D}_{6}\right) \delta_{P}-131.7$ (s). 


\section{Attempted chlorination of ${ }^{\mathrm{H}} \mathrm{PNP}$ using $\mathrm{PCl}_{5}$}

A three-necked flask under was connected to a bubbler containing $\mathrm{NEt}_{3}$. Into the flask was added $\mathrm{PCl}_{5}(2.74 \mathrm{~g}, 13.2 \mathrm{mmol})$ and diethyl ether $(15 \mathrm{~mL})$. The cloudy solution was cooled in ice then a freshly prepared ethereal solution of ${ }^{\mathrm{H}} \mathrm{PNP}$ was added slowly via cannula. After two hours the solvent was removed leaving a white solid residue. Samples of the crude solid were dissolved in both $\mathrm{C}_{6} \mathrm{D}_{6}$ and $\mathrm{CDCl}_{3}$ but no ${ }^{31} \mathrm{P}\left\{{ }^{1} \mathrm{H}\right\}$ NMR signals were visible in either spectrum.

\section{1,3- $\left[(2-\mathrm{pyr})_{2} \mathrm{P}(\mathrm{Se}) \mathrm{CH}_{2}\right]_{2} \mathrm{C}_{5} \mathrm{H}_{3} \mathrm{~N}\left({ }^{2-\mathrm{pyr}} \mathrm{PNP}-\mathrm{Se}_{2}, 26\right)$}

${ }^{2-p y r}$ PNP (82 mg, $0.17 \mathrm{mmol}$ ) was dissolved in chloroform $(5 \mathrm{~mL})$. Excess selenium powder (grey selenium, $176 \mathrm{mg}, 2.2 \mathrm{mmol}$ ) was added and the reaction refluxed overnight. The solution was cooled, the selenium filtered, and the chloroform removed under reduced pressure to give ${ }^{2-\text { pyr }} \mathrm{PNPSe}_{2}$ (26). Tan air-sensitive solid, $94 \%$.

${ }^{1} \mathrm{H}$ NMR: (300 MHz, $\left.\mathrm{CDCl}_{3}\right) \delta_{H} 8.70(\mathrm{~m}, 4 \mathrm{H}$, pyr-3), 8.19 (m, 4H, pyr-5), 7.71 (m, $4 \mathrm{H}$, pyr-6), 7.37 (t, ${ }^{3} J_{\mathrm{H}-\mathrm{H}}=8.6 \mathrm{~Hz}, 1 \mathrm{H}$, pyridine-4), $7.28(\mathrm{~m}, 4 \mathrm{H}$, pyr-4), $7.12(\mathrm{~d}$, ${ }^{3} J_{\mathrm{H}-\mathrm{H}}=7.6 \mathrm{~Hz}, 2 \mathrm{H}$, pyridine-3,5), $4.41\left(\mathrm{~s}, 4 \mathrm{H},{ }^{2} J_{\mathrm{P}-\mathrm{H}}=15.3 \mathrm{~Hz}, 4 \mathrm{H}, \mathrm{P}-\mathrm{CH}_{2}\right) \cdot{ }^{13} \mathrm{C}\left\{{ }^{1} \mathrm{H}\right\}$ NMR: $\left(125 \mathrm{MHz}, \mathrm{CDCl}_{3}\right) \delta_{C} 154.8\left(\mathrm{~d}, J_{\mathrm{P}-\mathrm{C}}=102.0 \mathrm{~Hz}\right.$, pyr-1), 152.3 (m, pyridine-2,6), $149.7\left(\mathrm{~d}, J_{\mathrm{P}-\mathrm{C}}=18.4 \mathrm{~Hz}\right.$, pyr-3), $136.5\left(\mathrm{~d}, J_{\mathrm{P}-\mathrm{C}}=10.9 \mathrm{~Hz}\right.$, pyr-5), 135.9 (s, pyridine-4), $129.1\left(\mathrm{~d}, J_{\mathrm{P}-\mathrm{C}}=26.2 \mathrm{~Hz}\right.$, pyr-6), $125.1\left(\mathrm{~d}, J_{\mathrm{P}-\mathrm{C}}=2.9 \mathrm{~Hz}\right.$, pyr-4), 123.5 (vt, ${ }^{3} J_{\mathrm{P}-\mathrm{C}}+$ ${ }^{5} J_{\mathrm{P}-\mathrm{C}}=3.93 \mathrm{~Hz}$, pyridine-3,5), $39.2\left(\mathrm{~d},{ }^{1} J_{\mathrm{P}-\mathrm{C}}=45.4 \mathrm{~Hz}, \mathrm{P}-\mathrm{CH}_{2}\right) .{ }^{31} \mathrm{P}\left\{{ }^{1} \mathrm{H}\right\} \mathrm{NMR}$ : $\left(121 \mathrm{MHz}, \mathrm{CDCl}_{3}\right) \delta_{P} 32.78\left(\mathrm{~s},{ }^{1} J_{\mathrm{P}-\mathrm{Se}}=742.0 \mathrm{~Hz}\right.$ ). HRMS calcd for $\mathrm{C}_{27} \mathrm{H}_{24} \mathrm{~N}_{5} \mathrm{P}_{2} \mathrm{Se}_{2}$ $[\mathrm{M}+\mathrm{H}]^{+}: m / z=637.9848$; found: 637.9851 .

\section{$1,3-\left[\mathrm{Ph}_{2} \mathrm{P}(\mathrm{Se}) \mathrm{CH}_{2}\right]_{2} \mathrm{C}_{5} \mathrm{H}_{3} \mathrm{~N}\left({ }^{\mathrm{Ph}} \mathrm{PNP}-\mathrm{Se}_{2}, 27\right)$}

${ }^{\mathrm{Ph}} \mathrm{PNP}$ (32.0 mg, $0.067 \mathrm{mmol}$ ) was dissolved in chloroform $(5 \mathrm{~mL})$. Excess selenium powder (grey selenium; $69.0 \mathrm{mg}, 0.875 \mathrm{mmol}$ ) was added and the reaction refluxed overnight. The solution was cooled, the selenium filtered, and the chloroform removed

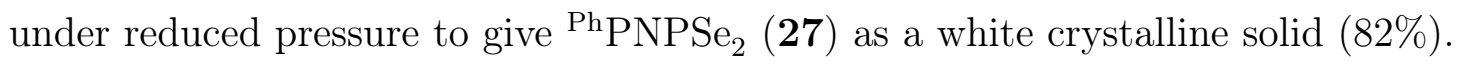

${ }^{1} \mathrm{H}$ NMR: $\left(500 \mathrm{MHz}, \mathrm{CDCl}_{3}\right) \delta_{H} 7.79\left(\mathrm{dd},{ }^{3} J_{\mathrm{P}-\mathrm{C}}=13.5 \mathrm{~Hz},{ }^{3} J_{\mathrm{H}-\mathrm{H}}=7.71 \mathrm{~Hz}, 8 \mathrm{H}\right.$, ortho$\mathrm{Ph}), 7.47$ (m, 4H, para- $\mathrm{Ph}$ ), 7.41 (m, 8H, meta- $\mathrm{Ph}$ ), 7.40 (overlap with meta- $\mathrm{Ph}, 1 \mathrm{H}$, pyridine-4), 7.15 (d, ${ }^{3} J_{\mathrm{H}-\mathrm{H}}=7.7 \mathrm{~Hz}, 2 \mathrm{H}$, pyridine-3,5), $3.98\left(\mathrm{~d},{ }^{2} J_{\mathrm{P}-\mathrm{H}}=14.3 \mathrm{~Hz}, 4 \mathrm{H}\right.$, $\left.\mathrm{P}-\mathrm{CH}_{2}\right) .{ }^{13} \mathrm{C}\left\{{ }^{1} \mathrm{H}\right\}$ NMR: $\left(125 \mathrm{MHz}, \mathrm{CDCl}_{3}\right) \delta_{\mathrm{C}} 151.7\left(\mathrm{~d},{ }^{2} J_{\mathrm{P}-\mathrm{C}}=8.7 \mathrm{~Hz}\right.$, pyridine- 
2,6), 135.6 (vt, $J_{\mathrm{P}-\mathrm{C}}=5.27 \mathrm{~Hz}$, pyridine-4), 132.2 (d, ${ }^{2} J_{\mathrm{P}-\mathrm{C}}=10.5 \mathrm{~Hz}$, ortho-Ph), 131.5 (m, para-Ph), 131.0 (s, ipso-Ph), 128.3 (d, ${ }^{3} J_{\mathrm{P}-\mathrm{C}}=12.4 \mathrm{~Hz}$, meta-Ph), 123.8 (vt, ${ }^{3} J_{\mathrm{P}-\mathrm{C}}$ $+{ }^{5} J_{\mathrm{P}-\mathrm{C}}=7.6 \mathrm{~Hz}$, pyridine-3,5), $43.2\left(\mathrm{~d},{ }^{1} J_{\mathrm{P}-\mathrm{C}}=42.6 \mathrm{~Hz}, \mathrm{P}-\mathrm{CH}_{2}\right) .{ }^{31} \mathrm{P}\left\{{ }^{1} \mathrm{H}\right\} \mathrm{NMR}$ : $\left(121 \mathrm{MHz}, \mathrm{CDCl}_{3}\right) \delta_{P} 33.11\left(\mathrm{~s},{ }^{1} J_{\mathrm{P}-\mathrm{Se}}=732.0 \mathrm{~Hz}\right.$ ). HRMS calcd for $\mathrm{C}_{31} \mathrm{H}_{28} \mathrm{NP}_{2} \mathrm{Se}_{2}$ $[\mathrm{M}+\mathrm{H}]^{+}: m / z=632.0051$; found: 632.0045. Anal. calcd for $\mathrm{C}_{31} \mathrm{H}_{27} \mathrm{NP}_{2} \mathrm{Se}_{2}: \mathrm{C}, 58.72$; H, 4.30; N, 2.21; found: C, 58.82; H, 4.27; N, 2.28 .

\section{$1,3-\left[{ }^{t} \mathrm{Bu}_{2} \mathrm{P}(\mathrm{Se}) \mathrm{CH}_{2}\right]_{2} \mathrm{C}_{5} \mathrm{H}_{3} \mathrm{~N}\left({ }^{t} \mathrm{Bu}^{\mathrm{PNP}}-\mathrm{Se}_{2}, 28\right)$}

${ }^{t}$ BuPNP (36.0 mg, $0.0910 \mathrm{mmol}$ ) was dissolved in toluene $(5 \mathrm{~mL})$. Excess selenium powder (grey selenium, $93.0 \mathrm{mg}, 1.18 \mathrm{mmol}$ ) was added and the reaction refluxed overnight. The solution was cooled, the selenium filtered, and the toluene removed under reduced pressure. The white solid was washed with diethyl ether to give ${ }^{{ }^{P h}} \mathrm{PNPSe}_{2}(\mathbf{2 8})$ as a white crystalline solid, $60 \%$.

${ }^{1} \mathrm{H}$ NMR: $\left(500 \mathrm{MHz}, \mathrm{CDCl}_{3}\right) \delta_{H} 7.80\left(\mathrm{~d},{ }^{3} J_{\mathrm{H}-\mathrm{H}}=8.0 \mathrm{~Hz}, 2 \mathrm{H}\right.$, pyridine-3,5), 7.59 $\left(\mathrm{t},{ }^{3} J_{\mathrm{H}-\mathrm{H}}=8.0 \mathrm{~Hz}, 1 \mathrm{H}\right.$, pyridine-4), $3.60\left(\mathrm{~d},{ }^{2} J_{\mathrm{P}-\mathrm{H}}=11.9 \mathrm{~Hz}, 4 \mathrm{H}, \mathrm{P}-\mathrm{CH}_{2}\right), 1.35$ (d, $\left.{ }^{3} J_{\mathrm{P}-\mathrm{H}}=15.2 \mathrm{~Hz}, 36 \mathrm{H}, \mathrm{CH}_{3}\right) .{ }^{13} \mathrm{C}\left\{{ }^{1} \mathrm{H}\right\} \mathrm{NMR}:\left(125 \mathrm{MHz}, \mathrm{CDCl}_{3}\right) \delta_{C} 153.4\left(\mathrm{~d},{ }^{2} J_{\mathrm{P}-\mathrm{C}}=5.7\right.$ $\mathrm{Hz}$, pyridine-2,6), 135.5 (m, pyridine-4), 132.2 (d, ${ }^{2} J_{\mathrm{P}-\mathrm{C}}=10.5 \mathrm{~Hz}$, ortho-Ph), 131.5 (m, para-Ph), 131.1 (s, ipso-Ph), 128.2 (d, ${ }^{3} J_{\mathrm{P}-\mathrm{C}}=12.4 \mathrm{~Hz}$, meta $\left.-\mathrm{Ph}\right), 124.6$ (vt, ${ }^{3} J_{\mathrm{P}-\mathrm{C}}+{ }^{5} J_{\mathrm{P}-\mathrm{C}}=2.7 \mathrm{~Hz}$, pyridine-3,5), $38.1\left(\mathrm{~d},{ }^{1} J_{\mathrm{P}-\mathrm{C}}=32.7 \mathrm{~Hz}, \mathrm{P}-\mathrm{CH}_{2}\right), 34.0(\mathrm{~d}$, $\left.{ }^{P-C} J_{29.3}=, \mathrm{Hz} C \mathrm{CH}_{3}\right), 28.4\left(\mathrm{~s}, \mathrm{CH}_{3}\right) .{ }^{31} \mathrm{P}\left\{{ }^{1} \mathrm{H}\right\} \mathrm{NMR}:\left(121 \mathrm{MHz}, \mathrm{CDCl}_{3}\right) \delta_{P} 75.96(\mathrm{~s}$, ${ }^{1} J_{\mathrm{P}-\mathrm{Se}}=702.8 \mathrm{~Hz}$ ). HRMS calcd for $\mathrm{C}_{23} \mathrm{H}_{44} \mathrm{NP}_{2} \mathrm{Se}_{2}[\mathrm{M}+\mathrm{H}]^{+}: m / z=554.1289$; found: 554.1305. Anal. calcd for $\mathrm{C}_{23} \mathrm{H}_{43} \mathrm{NP}_{2} \mathrm{Se}_{2}$ : C, 49.91; H, 7.83; N, 2.53; found: C, 50.11; H, 8.09; N, 2.68 .

\subsection{Metal Complexes}

\section{$\left[\left({ }^{2-p y r} \mathrm{PNP}\right) \operatorname{Ir}(\operatorname{cod})\right] \mathrm{Cl}(33)$}

To a solution of ${ }^{2-p y r} \mathrm{PNP}$ (50.0 $\left.\mathrm{mg}, 0.100 \mathrm{mmol}\right)$ in chloroform $(0.9 \mathrm{~mL})$ was added $[\operatorname{Ir}(\operatorname{cod})(\mu-\mathrm{Cl})]_{2}(35.0 \mathrm{mg}, 0.0521 \mathrm{mmol})$, resulting in a colour-change from orange to dark red-brown. Diethyl ether $(15 \mathrm{~mL})$ was added to precipitate a pale brown solid. 33 was isolated by decantation and dried briefly under reduced pressure $(70.0 \mathrm{mg}$, $83 \%)$. 
${ }^{1} \mathrm{H}$ NMR: $\left(500 \mathrm{MHz}, \mathrm{CDCl}_{3}\right) \delta_{H} 8.85(\mathrm{~m}, 2 \mathrm{H}$, pyrA-3), $8.06(\mathrm{~m}, 2 \mathrm{H}$, pyrB-3), 7.95 $\left(\mathrm{m}, 2 \mathrm{H}\right.$, pyrA-6), $7.86\left(\mathrm{~m}, 2 \mathrm{H}\right.$, pyrA-5), $7.83\left(\mathrm{t},{ }^{3} J_{\mathrm{H}-\mathrm{H}}=7.9 \mathrm{~Hz}, 1 \mathrm{H}\right.$, pyridine- 4$), 7.71$ $\left(\mathrm{d},{ }^{3} J_{\mathrm{H}-\mathrm{H}}=7.9 \mathrm{~Hz}, 2 \mathrm{H}\right.$, pyridine-3,5), $7.42(\mathrm{~m}, 2 \mathrm{H}$, pyrB-6), 7.41 (m, 2H, pyrA-4), 7.27 (m, 2H, pyrB-5), 6.93 (m, 2H, pyrB-4), $5.05\left(\mathrm{~m}, 2 \mathrm{H}, \mathrm{P}-\mathrm{CH}_{a}\right), 4.89\left(\mathrm{~m}, 2 \mathrm{H}, \mathrm{P}-\mathrm{CH}_{b}\right)$, 4.37 (br s, 2H, cod-CH-A), 2.70 (br s, 2H, cod-CH-B), 2.29 (br s, 2H, cod-CH $\mathrm{CH}_{a} \mathrm{~B}$ ), 1.70 (br s, 2H, cod-CH$-\mathrm{CH}_{b}$-B), 1.34 (br s, $2 \mathrm{H}$, cod-CH $\mathrm{CH}_{a}-\mathrm{A}$ ), 0.86 (br s, $2 \mathrm{H}$, cod- $\mathrm{CH}_{b}-\mathrm{A}$ ). ${ }^{13} \mathrm{C}\left\{{ }^{1} \mathrm{H}\right\}$ NMR: $\left(125 \mathrm{MHz}, \mathrm{CDCl}_{3}\right) \delta_{C} 161.9\left(\mathrm{~d},{ }^{2} J_{\mathrm{P}-\mathrm{C}}=10.2 \mathrm{~Hz}\right.$, pyridine-2,6), 157.2 $\left(\mathrm{d},{ }^{1} J_{\mathrm{P}-\mathrm{C}}=44.4 \mathrm{~Hz}, \mathrm{pyrB}-1\right), 156.2\left(\mathrm{~d},{ }^{1} J_{\mathrm{P}-\mathrm{C}}=62.6 \mathrm{~Hz}, \mathrm{pyrA}-1\right), 150.4\left(\mathrm{~d},{ }^{3} J_{\mathrm{P}-\mathrm{C}}=14.9\right.$ $\mathrm{Hz}, \operatorname{pyrA}-3), 150.1$ (d, ${ }^{3} J_{\mathrm{P}-\mathrm{C}}=13.1 \mathrm{~Hz}$, pyrB-3), 139.5 (s, pyridine-4), 136.9 (d, ${ }^{3} J_{\mathrm{P}-\mathrm{C}}=7.2 \mathrm{~Hz}$, pyrA-5), $136.2\left(\mathrm{~d},{ }^{3} J_{\mathrm{P}-\mathrm{C}}=8.9 \mathrm{~Hz}\right.$, pyrB-5$), 128.2\left(\mathrm{~d},{ }^{2} J_{\mathrm{P}-\mathrm{C}}=19.3 \mathrm{~Hz}\right.$, pyrA-6), 127.8 (d, ${ }^{2} J_{\mathrm{P}-\mathrm{C}}=27.1 \mathrm{~Hz}$, pyrB-6), 124.4 (s, pyrA-4), 123.6 (s, pyrB-4), $122.3\left(\mathrm{~d},{ }^{3} J_{\mathrm{P}-\mathrm{C}}=9.9 \mathrm{~Hz}\right.$, pyridine-3,5), $72.3(\mathrm{~s}, \operatorname{cod}-\mathrm{CH}), 59.1\left(\mathrm{~d},{ }^{2} J_{\mathrm{P}-\mathrm{C}}=26.8 \mathrm{~Hz}\right.$, cod-CH), $44.9\left(\mathrm{~d},{ }^{1} J_{\mathrm{P}-\mathrm{C}}=36.2 \mathrm{~Hz}, \mathrm{P}-\mathrm{CH}_{2}\right), 32.0$ (m, cod-CH2). ${ }^{31} \mathrm{P}\left\{{ }^{1} \mathrm{H}\right\}$ NMR: $(121$ $\mathrm{MHz}, \mathrm{CDCl}_{3}$ ) $\delta_{P} 13.77$ (s). ${ }^{15} \mathrm{~N}$ NMR: (60 $\mathrm{MHz} \mathrm{CDCl}_{3}$ ) $\delta_{N}-60.4$ (pyrA), -61.5 (pyrB), -152.6 (pyridine) HRMS calcd for $\mathrm{C}_{35} \mathrm{H}_{35} \mathrm{IrN}_{5} \mathrm{P}_{2}[\mathrm{M}-\mathrm{Cl}]^{+}: \mathrm{m} / z=780.1997$; found: 780.2041. HRMS calcd for $\mathrm{C}_{35} \mathrm{H}_{36} \mathrm{IrN}_{5} \mathrm{P}_{2}[\mathrm{M}-\mathrm{Cl}+\mathrm{H}]^{+}: m / z=781.2075$; found: 781.2057. Anal. calcd for $\mathrm{C}_{35} \mathrm{H}_{35} \mathrm{ClIrN}_{5} \mathrm{P}_{2}+2 \mathrm{H}_{2} \mathrm{O}+0.2 \mathrm{CHCl}_{3}{ }^{*}$ : C, 47.79; H, 4.47; N, 7.89. Found: C, 48.21; H, 4.59; N, 7.51.

\section{${ }^{2-p y r} \mathrm{PNP}+[\operatorname{Ir}(\operatorname{cod})(\mu-\mathrm{OMe})]_{2}$}

To a solution of ${ }^{2-p y r}$ PNP $(28.0 \mathrm{mg}, 0.0588 \mathrm{mmol})$ in chloroform $(0.4 \mathrm{~mL})$ was added $[\operatorname{Ir}(\operatorname{cod})(\mu-\mathrm{OMe})]_{2}(19.0 \mathrm{mg}, 0.0300 \mathrm{mmol})$, resulting in a dark red-brown solution. $4 \mathrm{~mL}$ diethyl ether was added to precipitate a pale brown solid. The product was isolated by decantation and dried briefly under reduced pressure.

${ }^{1} \mathrm{H}$ NMR: (500 MHz, $\left.\mathrm{CDCl}_{3}\right) \delta_{H} 8.82(\mathrm{~m}, 2 \mathrm{H}$, pyrA-3), 8.01 (m, 2H, pyrB-3), 7.84 $\left(\mathrm{m}, 2 \mathrm{H}\right.$, pyrA-6), $7.83\left(\mathrm{~m}, 2 \mathrm{H}\right.$, pyrA-5), $7.81\left(\mathrm{t},{ }^{3} J_{\mathrm{H}-\mathrm{H}}=8.0 \mathrm{~Hz}, 1 \mathrm{H}\right.$, pyridine- 4$), 7.66$ $\left(\mathrm{d},{ }^{3} J_{\mathrm{H}-\mathrm{H}}=7.6 \mathrm{~Hz}, 2 \mathrm{H}\right.$, pyridine-3,5), 7.38 (m, 2H, pyrA-4), 7.26 (m, 2H, pyrB-6), $7.23\left(\mathrm{~m}, 2 \mathrm{H}\right.$, pyrB-5), $6.91\left(\mathrm{~m}, 2 \mathrm{H}\right.$, pyrB-4), $4.99\left(\mathrm{~m}, 0.02 \mathrm{H}, \mathrm{P}-\mathrm{CH}_{a}\right), 4.81(\mathrm{~m}$, $0.04 \mathrm{H}, \mathrm{P}-\mathrm{CH}_{b}$ ), 4.35 (br s, 2H, cod-CH-A), 2.71 (br s, 2H, cod-CH-B), 2.30 (br s, $2 \mathrm{H}$, cod- $\mathrm{CH}_{a}-\mathrm{B}$ ), 1.71 (br s, 2H, cod- $\mathrm{CH}_{b}-\mathrm{B}$ ), 1.34 (br s, $2 \mathrm{H}$, cod- $\mathrm{CH}_{a}-\mathrm{A}$ ), 0.88 (br s, $2 \mathrm{H}$, cod- $\left.\mathrm{CH}_{b}-\mathrm{A}\right) .{ }^{13} \mathrm{C}\left\{{ }^{1} \mathrm{H}\right\}$ NMR: $\left(125 \mathrm{MHz}, \mathrm{CDCl}_{3}\right) \delta_{C} 162.0\left(\mathrm{~d},{ }^{2} J_{\mathrm{P}-\mathrm{C}}=8.9\right.$ $\mathrm{Hz}$, pyridine-2,6), 157.0 (d, ${ }^{1} J_{\mathrm{P}-\mathrm{C}}=44.4 \mathrm{~Hz}$, pyrB-1), 156.0 (d, ${ }^{1} J_{\mathrm{P}-\mathrm{C}}=63.1 \mathrm{~Hz}$, pyrA1), $150.3\left(\mathrm{~d},{ }^{3} J_{\mathrm{P}-\mathrm{C}}=14.9 \mathrm{~Hz}\right.$, pyrA-3), 150.1 (d, ${ }^{3} J_{\mathrm{P}-\mathrm{C}}=13.2 \mathrm{~Hz}$, pyrB-3), 139.5 ( $\mathrm{s}$, pyridine-4), 136.9 (d, ${ }^{3} J_{\mathrm{P}-\mathrm{C}}=7.2 \mathrm{~Hz}$, pyrA-5), 136.1 (d, ${ }^{3} J_{\mathrm{P}-\mathrm{C}}=8.3 \mathrm{~Hz}$, pyrB-5), 127.8 $\left(\mathrm{d},{ }^{2} J_{\mathrm{P}-\mathrm{C}}=18.7 \mathrm{~Hz}\right.$, pyrA-6), $127.4\left(\mathrm{~d},{ }^{2} J_{\mathrm{P}-\mathrm{C}}=27.0 \mathrm{~Hz}\right.$, pyrB-6), 124.4 (s, pyrA-4), $123.6\left(\mathrm{~s}\right.$, pyrB-4), $122.2\left(\mathrm{~d},{ }^{3} J_{\mathrm{P}-\mathrm{C}}=9.2 \mathrm{~Hz}\right.$, pyridine-3,5), 72.1 (s, cod-CH), 59.0 (d,

\footnotetext{
${ }^{*}$ It is difficult to dry $\mathbf{3 3}$ without formation of an insoluble product.
} 
${ }^{2} J_{\mathrm{P}-\mathrm{C}}=28.3 \mathrm{~Hz}$, cod-CH), 44.6 (br m, P- $\mathrm{CH}_{2}$ ), 31.8 (m, cod-CH2). ${ }^{31} \mathrm{P}\left\{{ }^{1} \mathrm{H}\right\} \mathrm{NMR}$ : $\left(202 \mathrm{MHz}, \mathrm{CDCl}_{3}\right) \delta_{p} 12.78(\mathrm{~s})$.

\section{$\left[\left({ }^{2-p y r} \mathrm{PNP}\right) \operatorname{Ir}(\operatorname{cod})\right] \mathrm{BPh}_{4}$}

${ }^{1} \mathrm{H}$ NMR: $\left(500 \mathrm{MHz}, \mathrm{CDCl}_{3}\right) \delta_{H} 8.80(\mathrm{~m}, 2 \mathrm{H}$, pyrA-3), $7.94(\mathrm{~m}, 2 \mathrm{H}$, pyrB-3), 7.66 (m, 2H, pyrA-5), 7.44 (m, 8H, meta-Ph), 7.39 (m, 2H, pyrA), 7.39 (m, 2H, pyrA), 7.39 (t (overlapping), 1H, pyridine-4), 7.30 (m, 2H, pyrB), 7.11 (d, ${ }^{3} J_{\mathrm{H}-\mathrm{H}}=7.8 \mathrm{~Hz}$, $2 \mathrm{H}$, pyridine-3,5), $7.01(\mathrm{~m}, 2 \mathrm{H}, \mathrm{pyrB}), 6.93\left(\mathrm{t},{ }^{3} J_{\mathrm{H}-\mathrm{H}}=7.2 \mathrm{~Hz}, 8 \mathrm{H}\right.$, ortho- $\left.\mathrm{Ph}\right), 6.80$ $\left(\mathrm{m}, 2 \mathrm{H}\right.$, pyrB-4), $6.75\left(\mathrm{t},{ }^{3} J_{\mathrm{H}-\mathrm{H}}=7.2 \mathrm{~Hz}, 4 \mathrm{H}\right.$, para $\left.-\mathrm{Ph}\right), 4.60\left(\mathrm{~m}, 2 \mathrm{H}, \mathrm{P}-\mathrm{CH}_{a}\right), 4.31$ $\left(\mathrm{m}, 2 \mathrm{H}, \mathrm{P}-\mathrm{CH}_{b}\right.$ ), 4.30 (br s, 2H, cod-CH-A), 2.61 (br s, 2H, cod-CH-B), 2.24 (br s, $2 \mathrm{H}$, cod- $\mathrm{CH}_{a}-\mathrm{B}$ ), 1.70 (br s, 2H, cod- $\mathrm{CH}_{b}-\mathrm{B}$ ), 1.30 (br s, 2H, cod-CH ${ }_{a}-\mathrm{A}$ ), 0.82 (br s, $2 \mathrm{H}$, cod- $\left.\mathrm{CH}_{b}-\mathrm{A}\right) .{ }^{31} \mathrm{P}\left\{{ }^{1} \mathrm{H}\right\}$ NMR: (121 MHz, $\left.\mathrm{CDCl}_{3}\right) \delta_{p} 12.30$ (s).

\section{$\left[\left({ }^{\mathrm{Ph}} \mathrm{PNP}\right) \operatorname{Ir}(\operatorname{cod})\right] \mathrm{Cl}(35)$}

${ }^{\mathrm{Ph}} \mathrm{PNP}(67.0 \mathrm{mg}, 0.141 \mathrm{mmol})$ and $[\mathrm{Ir}(\operatorname{cod})(\mu-\mathrm{Cl})]_{2}(47.4 \mathrm{mg}, 0.0700 \mathrm{mmol})$ were combined in $\mathrm{CHCl}_{3}(3 \mathrm{~mL})$. The orange solution was then dried under reduced pressure, and the resulting solid washed with pentane $(3 \mathrm{~mL})$. After drying under reduced pressure $\mathbf{3 5}$ was obtained as a yellow solid (88 $\mathrm{mg}, 77 \%$ ).

${ }^{1} \mathrm{H}$ NMR: $\left(500 \mathrm{MHz}, \mathrm{CDCl}_{3}\right) \delta_{H} 7.94\left(\mathrm{~d},{ }^{3} J_{\mathrm{H}-\mathrm{H}}=8.1 \mathrm{~Hz}, 2 \mathrm{H}\right.$, pyridine-3,5), $7.85(\mathrm{t}$, ${ }^{3} J_{\mathrm{H}-\mathrm{H}}=7.9 \mathrm{~Hz}, 1 \mathrm{H}$, pyridine-4), $7.76(\mathrm{t}, J=8.9 \mathrm{~Hz}, 4 \mathrm{H}$, ortho-PhA), 7.59 (m, 4H, meta-PhA), $7.53(\mathrm{~m}, 2 \mathrm{H}$, para- $\mathrm{PhA}), 7.10\left(\mathrm{t},{ }^{3} J_{\mathrm{H}-\mathrm{H}}=7.8 \mathrm{~Hz}, 2 \mathrm{H}\right.$, para- $\left.\mathrm{PhB}\right), 6.84(\mathrm{t}$, J3H-H7.6, 4H, meta-PhB), $6.76\left(\mathrm{t}, J=9.9 \mathrm{~Hz}\right.$, ortho-PhB), $4.82\left(\mathrm{~m}, 2 \mathrm{H}, \mathrm{P}-\mathrm{CH}_{a}\right)$, $4.70\left(\mathrm{~m}, 2 \mathrm{H}, \mathrm{P}-\mathrm{CH}_{b}\right), 3.96$ (br s, 2H, cod-CH-A), 2.69 (br s, 2H, cod-CH-B), 2.17 (br s, 2H, cod-CH $\mathrm{CH}_{a} \mathrm{~B}$ ), 1.60 (br s, 2H, cod- $\mathrm{CH}_{b}$-B), 1.19 (br s, 2H, cod-CH $\mathrm{CH}_{a}-\mathrm{A}$ ), 0.93 (br s, $2 \mathrm{H}$, cod- $\left.\mathrm{CH}_{b}-\mathrm{A}\right) \cdot{ }^{13} \mathrm{C}\left\{{ }^{1} \mathrm{H}\right\}$ NMR: $\left(125 \mathrm{MHz}, \mathrm{CDCl}_{3}\right) \delta_{C} 161.0\left(\mathrm{~d},{ }^{2} J_{\mathrm{P}-\mathrm{C}}=8.5\right.$ $\mathrm{Hz}$, pyridine-2,6), 140.1 (s, pyridine-4), 132.7 (d, ${ }^{1} J_{\mathrm{P}-\mathrm{C}}=24.2 \mathrm{~Hz}$, ipso-PhB), 132.3 (d, ${ }^{1} J_{\mathrm{P}-\mathrm{C}}=42.4 \mathrm{~Hz}$, ipso-PhA), $131.6\left(\mathrm{~d},{ }^{4} J_{\mathrm{P}-\mathrm{C}}=12.7 \mathrm{~Hz}\right.$, ortho-PhB $), 131.0\left(\mathrm{~d},{ }^{4} J_{\mathrm{P}-\mathrm{C}}=8.9\right.$ $\mathrm{Hz}$, ortho-PhA), 130.5 (s, para- $\mathrm{PhB}), 130.2$ (s, para-PhA), 129.4 (d, ${ }^{2} J_{\mathrm{P}-\mathrm{C}}=9.6 \mathrm{~Hz}$ meta-PhA), 128.6 (d, ${ }^{2} J_{\mathrm{P}-\mathrm{C}}=9.6 \mathrm{~Hz}$, meta-PhB), 123.9 (d, ${ }^{3} J_{\mathrm{P}-\mathrm{C}}=8.6 \mathrm{~Hz}$, pyridine3,5), 72.1 (s, cod-CH), $58.6\left(\mathrm{~d},{ }^{2} J_{\mathrm{P}-\mathrm{C}}=27.8 \mathrm{~Hz}, \operatorname{cod}-\mathrm{CH}\right), 46.8\left(\mathrm{~d},{ }^{1} J_{\mathrm{P}-\mathrm{C}}=32.6 \mathrm{~Hz}\right.$, $\left.\mathrm{P}-\mathrm{CH}_{2}\right), 31.4$ (m, cod-CH2). ${ }^{31} \mathrm{P}\left\{{ }^{1} \mathrm{H}\right\} \mathrm{NMR}$ : (121 MHz, $\left.\mathrm{CDCl}_{3}\right) \delta_{P} 11.04(\mathrm{~s})$. 


\section{$\left[\left({ }^{2-p y r} \mathrm{PNP}\right) \operatorname{Ir}\left(\mathrm{C}_{2} \mathrm{H}_{4}\right)_{2}\right] \mathrm{Cl}(36)$}

To a solution of ${ }^{2-p y r}$ PNP (30.0 mg, $\left.0.0626 \mathrm{mmol}\right)$ in chloroform $(2 \mathrm{~mL})$ was added $[\operatorname{Ir}(\operatorname{cod})(\mu-\mathrm{Cl})]_{2}(21.0 \mathrm{mg}, 0.0313 \mathrm{mmol})$, resulting in a colour-change from orange to dark red-brown that indicates the formation of $\mathbf{3 3}$. The solvent was removed and $\mathbf{3 3}$ dissolved in 1:1 $\mathrm{D}_{2} \mathrm{O} /$ acetone- $\mathrm{d}_{6}$. This solution was transferred to a Fisher-Porter tube and pressurised with ethene to 5 atm. After 5 days the pressure was released and the solution transferred to a Schlenk tube under ethene.

For variable temperature NMR studies 36 was synthesised in $\mathrm{CD}_{2} \mathrm{Cl}_{2}$. Spectra collected in $\mathrm{D}_{2} \mathrm{O} /$ acetone- $\mathrm{d}_{6}$ are referenced to acetone at $\delta_{H} 2.22 \mathrm{ppm}$.

${ }^{1} \mathrm{H}$ NMR: $\left(500 \mathrm{MHz}, 20{ }^{\circ} \mathrm{C}, \mathbf{D}_{\mathbf{2}} \mathbf{O} /\right.$ acetone-d $\left.{ }_{6}\right) \delta_{H} 8.75(\mathrm{~m}, 4 \mathrm{H}$, pyr-3), $8.23(\mathrm{t}$, ${ }^{3} J_{\mathrm{H}-\mathrm{H}}=8.1 \mathrm{~Hz}, 1 \mathrm{H}$, pyridine-4), $8.05\left(\mathrm{~d},{ }^{3} J_{\mathrm{H}-\mathrm{H}}=8.1 \mathrm{~Hz}, 2 \mathrm{H}\right.$, pyridine-3,5), $7.95(\mathrm{~m}$, $4 \mathrm{H}$, pyr-5), $7.83\left(\mathrm{~m}, 4 \mathrm{H}\right.$, pyr-6), $7.56\left(\mathrm{~m}, 4 \mathrm{H}\right.$, pyr-4), $5.03\left(\mathrm{~m}, 4 \mathrm{H}, \mathrm{P}-\mathrm{CH}_{2}\right) \cdot{ }^{1} \mathrm{H}$ NMR: $\left(300 \mathrm{MHz}, 50{ }^{\circ} \mathrm{C}, \mathrm{D}_{2} \mathrm{O} /\right.$ acetone- $\left._{6}\right) \delta_{H} 2.10$ (br s, $\left.\mathrm{C}_{2} \mathrm{H}_{4}\right) .{ }^{13} \mathrm{C}\left\{{ }^{1} \mathrm{H}\right\} \mathrm{NMR}$ : $\left(125 \mathrm{MHz}, 20{ }^{\circ} \mathrm{C}, \mathrm{D}_{2} \mathrm{O} /\right.$ acetone- $\left.\mathrm{d}_{6}\right) \delta_{C} 164.7$ (m, pyridine-2,6), 152.9 (vt, ${ }^{3} J_{\mathrm{P}-\mathrm{C}}+$ ${ }^{5} J_{\mathrm{P}-\mathrm{C}}=7.4 \mathrm{~Hz}$, pyr-3), 151.0 (m, pyr-1), 141.9 (s, pyridine-4), 139.2 (m, pyr-5), 130.6 (br s, pyr-6), 127.9 (s, pyr-4), 123.8 (s, pyridine-3,5), 43.6 (br s, $\mathrm{P}-\mathrm{CH}_{2}$ ). ${ }^{31} \mathrm{P}\left\{{ }^{1} \mathrm{H}\right\}$ NMR: $\left(202 \mathrm{MHz}, \mathrm{D}_{2} \mathrm{O} /\right.$ acetone- $\left.\mathrm{d}_{6}\right) \delta_{P} 4.48(\mathrm{~m})$.

${ }^{1} \mathrm{H}$ NMR: $\left(300 \mathrm{MHz}, 20{ }^{\circ} \mathrm{C}, \mathbf{C D}_{\mathbf{2}} \mathbf{C l}_{\mathbf{2}}\right) \delta_{H} 8.67\left(\mathrm{~m}, 4 \mathrm{H}\right.$, pyr-3), $8.06\left(\mathrm{t},{ }^{3} J_{\mathrm{H}-\mathrm{H}}=7.8\right.$ $\mathrm{Hz}, 1 \mathrm{H}$, pyridine-4), $7.95\left(\mathrm{~d},{ }^{3} J_{\mathrm{H}-\mathrm{H}}=7.8 \mathrm{~Hz}, 2 \mathrm{H}\right.$, pyridine- 3,5$), 7.75(\mathrm{~m}, 8 \mathrm{H}$, pyr-5,6), 7.35 (m, 4H, pyr-4), 5.00 (vt, $\left.J=5.4 \mathrm{~Hz}, 4 \mathrm{H}, \mathrm{P}-\mathrm{CH}_{2}\right) .{ }^{1} \mathrm{H}$ NMR: $\left(300 \mathrm{MHz},-70{ }^{\circ} \mathrm{C}\right.$, $\mathrm{CD}_{2} \mathrm{Cl}_{2}$ ) $\delta_{H} 2.73$ (br s, $\left.4 \mathrm{H}, \mathrm{C}_{2} \mathrm{H}_{4}\right), 1.07$ (br s, $\left.4 \mathrm{H}, \mathrm{C}_{2} \mathrm{H}_{4}\right) \cdot{ }^{13} \mathrm{C}\left\{{ }^{1} \mathrm{H}\right\}$ NMR: $(75 \mathrm{MHz}$, $\left.-70{ }^{\circ} \mathrm{C}, \mathrm{CD}_{2} \mathrm{Cl}_{2}\right) \delta_{C} 162.5$ (s, pyridine-2,6), 150.3 (vt, ${ }^{3} J_{\mathrm{P}-\mathrm{C}}+{ }^{5} J_{\mathrm{P}-\mathrm{C}}=8.1 \mathrm{~Hz}$, pyr-3), 148.6 (vt, $J=38.1 \mathrm{~Hz}$, pyr-1), 139.0 (s, pyridine-4), 136.3 (s, pyr-5), 128.1 (vt, ${ }^{2} J_{\mathrm{P}-\mathrm{C}}$ $+{ }^{6} J_{\mathrm{P}-\mathrm{C}}=10.6 \mathrm{~Hz}$, pyr-6), 125.1 (s, pyr-4), 121.4 (vt, $J=5.2 \mathrm{~Hz}$, pyridine-3,5), 41.2 (vt, $\left.J=20.8 \mathrm{~Hz}, \mathrm{P}-\mathrm{CH}_{2}\right), 27.0\left(\mathrm{~s}, \mathrm{C}_{2} \mathrm{H}_{4}\right), 21.1\left(\mathrm{~s}, \mathrm{C}_{2} \mathrm{H}_{4}\right) .{ }^{31} \mathrm{P}\left\{{ }^{1} \mathrm{H}\right\} \mathrm{NMR}:(121 \mathrm{MHz}$, $\left.20{ }^{\circ} \mathrm{C}, \mathrm{CD}_{2} \mathrm{Cl}_{2}\right) \delta_{P} 3.97(\mathrm{~s}) .{ }^{15} \mathrm{~N}$ NMR: $\left(60 \mathrm{MHz}, 20{ }^{\circ} \mathrm{C}, \mathrm{CD}_{2} \mathrm{Cl}_{2}\right) \delta_{N}-58.3$ (pyr), -152.2 (pyridine).

\section{$\left[\left({ }^{2-p y r} \mathrm{PNP}\right) \operatorname{Ir}(\mathrm{H})_{2} \mathrm{Cl}\right] \mathrm{Cl}(37)$}

To a solution of ${ }^{2-p y r} \mathrm{PNP}(82.0 \mathrm{mg}, 0.171 \mathrm{mmol})$ in dichloromethane $(7 \mathrm{~mL})$ was added $[\operatorname{Ir}(\operatorname{cod})(\mu-\mathrm{Cl})]_{2}(57.0 \mathrm{mg}, 0.0849 \mathrm{mmol})$, resulting in a colour-change from orange to dark red-brown indicating the formation of 33. This solution was transferred to a Fisher-Porter tube and pressurised with hydrogen to $5 \mathrm{~atm}$. After 5 days the 
pressure was released and the orange solution was filtered through a plug made from glass fibre filter paper. The solution was concentrated and $n$-hexane $(5 \mathrm{~mL})$ added to precipitate the pale yellow solid. (72 mg, 59\%).

${ }^{1} \mathrm{H}$ NMR: (500 MHz, $\left.\mathrm{CD}_{2} \mathrm{Cl}_{2}\right) \delta_{H} 8.67$ (m, 2H, pyrA-3), $8.62(\mathrm{~m}, 2 \mathrm{H}$, pyrB-3), 8.41 (m, 2H, pyrA-6), 7.85 (m, 2H, pyrB-6), 7.81 (m, 2H, pyrA-5), 7.70 (t, ${ }^{3} J_{\mathrm{H}-\mathrm{H}}=7.8 \mathrm{~Hz}$, 1H, pyridine-4), $7.68\left(\mathrm{~m}, 2 \mathrm{H}\right.$, pyrB-5), $7.49\left(\mathrm{~d},{ }^{3} J_{\mathrm{H}-\mathrm{H}}=7.8 \mathrm{~Hz}, 2 \mathrm{H}\right.$, pyridine-3,5), 7.33 (m, 2H, pyrA-4), 7.27 (m, 2H, pyrB-4), 4.85 (vt, $J=5.3 \mathrm{~Hz}, 4 \mathrm{H}, \mathrm{P}-\mathrm{CH}_{2}$ ), -18.34 (td, $\left.{ }^{2} J_{\mathrm{P}-\mathrm{H}}=13.5 \mathrm{~Hz},{ }^{2} J_{\mathrm{H}-\mathrm{H}}=7.67 \mathrm{~Hz}, 1 \mathrm{H}, \mathrm{Ir}-\mathrm{H}\right),-21.56\left(\mathrm{td},{ }^{2} J_{\mathrm{P}-\mathrm{H}}=16.8 \mathrm{~Hz},{ }^{2} J_{\mathrm{H}-\mathrm{H}}=7.29 \mathrm{~Hz}\right.$, $1 \mathrm{H}, \mathrm{Ir}-\mathrm{H}) .{ }^{13} \mathrm{C}\left\{{ }^{1} \mathrm{H}\right\}$ NMR: (125 MHz, $\left.\mathrm{CD}_{2} \mathrm{Cl}_{2}\right) \delta_{C} 162.3$ (vt, $J=5.1 \mathrm{~Hz}$, pyridine-2,6). 160.5 (vt, $J=39.0 \mathrm{~Hz}$, pyrB-1), 155.2 (vt, $J=34.6 \mathrm{~Hz}$, pyrA-1), 150.3 (vt, ${ }^{3} J_{\mathrm{P}-\mathrm{C}}$ $+{ }^{5} J_{\mathrm{P}-\mathrm{C}}=6.7 \mathrm{~Hz}$, pyrA-3), $149.9\left(\mathrm{vt},{ }^{3} J_{\mathrm{P}-\mathrm{C}}+{ }^{5} J_{\mathrm{P}-\mathrm{C}}=6.7 \mathrm{~Hz}\right.$, pyrB-3), $136.6(\mathrm{~m}$, pyridine-4), 136.3 (vt, ${ }^{3} J_{\mathrm{P}-\mathrm{C}}+{ }^{5} J_{\mathrm{P}-\mathrm{C}}=4.7 \mathrm{~Hz}$, pyrB-5), 136.1 (vt, ${ }^{3} J_{\mathrm{P}-\mathrm{C}}+{ }^{5} J_{\mathrm{P}-\mathrm{C}}=5.5$ Hz, pyrA-5), 132.5 (vt, $J=16.5 \mathrm{~Hz}$, pyrA-6), 129.7 (vt, $J=14.9 \mathrm{~Hz}$, pyrB-6), 124.7 (s, pyrA-4), 124.2 (s, pyrB-4), 121.0 (vt, ${ }^{3} J_{\mathrm{P}-\mathrm{C}}+{ }^{5} J_{\mathrm{P}-\mathrm{C}}=6.0 \mathrm{~Hz}$, pyridine-3,5), 43.80 (vt, $\left.J=19.7 \mathrm{~Hz}, \mathrm{P}-\mathrm{CH}_{2}\right) .{ }^{31} \mathrm{P}\left\{{ }^{1} \mathrm{H}\right\} \mathrm{NMR}:\left(202 \mathrm{MHz}, \mathrm{CD}_{2} \mathrm{Cl}_{2}\right) \delta_{P} 26.86(\mathrm{~m}) .{ }^{15} \mathrm{~N}$ NMR: $\left(60 \mathrm{MHz}, \mathrm{CDCl}_{3}\right.$ ) $\delta_{N}-60.4$ (pyrA), -62.9 (pyrB), -120.8 (pyridine). HRMS calcd for $\mathrm{C}_{27} \mathrm{H}_{25} \mathrm{IrN}_{5} \mathrm{P}_{2}[\mathrm{M}-\mathrm{Cl}]^{+}: \mathrm{m} / z=674.1214$; found: 674.1205 . HRMS calcd for $\mathrm{C}_{27} \mathrm{H}_{26} \mathrm{ClIrN}_{5} \mathrm{P}_{2}[\mathrm{M}+\mathrm{H}]^{+}: m / z=709.0903$; found: 709.0936. IR (KBr): $\nu_{\max } / \mathrm{cm}^{-1}$ $=2180,2083(\mathrm{Ir}-\mathrm{H})$.

\section{$\left[\left({ }^{2-p y r} \mathrm{PNP}\right) \mathrm{RhCl}_{3}\right.$}

To a solution of ${ }^{2-p y r} \mathrm{PNP}(20 \mathrm{mg}, 0.042 \mathrm{mmol})$ in $\mathrm{CDCl}_{3}$ was added $[\mathrm{Rh}(\operatorname{cod})(\mu-\mathrm{Cl})]_{2}$ (10.3 $\mathrm{mg}, 0.0210 \mathrm{mmol}$ ). NMR spectra of the result were obtained, then the turbid orange solution was allowed to settle. The solution was then decanted and poured into diethyl ether. The resulting tan solid was filtered, washed with hexane, then dried under vacuum for 3 hours to yield $3.4 \mathrm{mg}$. The dark red chloroform-insoluble material $(21 \mathrm{mg})$ is insoluble in water and water/acetone.

${ }^{1} \mathrm{H}$ NMR: $\left(500 \mathrm{MHz}, \mathrm{CDCl}_{3}\right) \delta_{H} 8.68$ (m, pyr-3), 8.66 (m, pyr-6), 7.77 (m, pyr-5), $7.68\left(\mathrm{t},{ }^{3} J_{\mathrm{H}-\mathrm{H}}=7.8 \mathrm{~Hz}, 1 \mathrm{H}\right.$, pyridine-4), $7.56\left(\mathrm{~d},{ }^{3} J_{\mathrm{H}-\mathrm{H}}=7.8 \mathrm{~Hz}, 2 \mathrm{H}\right.$, pyridine- 3,5$), 7.32$ (m, pyr-4), 5.25 (vt, $\left.J=5.9 \mathrm{~Hz}, 4 \mathrm{H}, \mathrm{P}-\mathrm{CH}_{2}\right) \cdot{ }^{13} \mathrm{C}\left\{{ }^{1} \mathrm{H}\right\} \mathrm{NMR}:\left(150 \mathrm{MHz}, \mathrm{CDCl}_{3}\right)$ $\delta_{C} 163.3$ (m, pyridine-2,6), 152.2 (m, pyr-1), 149.7 (vt, $J=6.8 \mathrm{~Hz}$, pyr-3), 138.6 (s, pyridine-4), 136.8 (s, pyr-5), 132.4 (vt, ${ }^{2} J_{\mathrm{P}-\mathrm{C}}+{ }^{6} J_{\mathrm{P}-\mathrm{C}}=14.4 \mathrm{~Hz}$, pyr-6), 125.2 (s, pyr4), 123.3 (m, pyridine-3,5), 37.5 (vt, $\left.J=17.8 \mathrm{~Hz}, \mathrm{P}-\mathrm{CH}_{2}\right) .{ }^{31} \mathrm{P}\left\{{ }^{1} \mathrm{H}\right\} \mathrm{NMR}$ : (202 MHz, $\left.\mathrm{CDCl}_{3}\right) \delta_{P} 29.22\left(\mathrm{~d},{ }^{1} J_{\mathrm{Rh}-\mathrm{P}}=92.6 \mathrm{~Hz}\right)$. HRMS calcd for $\mathrm{C}_{27} \mathrm{H}_{24} \mathrm{Cl}_{3} \mathrm{~N}_{5} \mathrm{P}_{2} \mathrm{Rh}[\mathrm{M}+\mathrm{H}]^{+}$: $m / z=687.9628$; found: 687.9718. Anal. calcd for $\mathrm{C}_{27} \mathrm{H}_{23} \mathrm{Cl}_{3} \mathrm{~N}_{5} \mathrm{P}_{2} \mathrm{Rh}+1.5 \mathrm{H}_{2} \mathrm{O}$ : $\mathrm{C}$, 45.31; H, 3.66; N, 9.78. Found: C, 45.47; H, 3.87; N, 9.51. 


\subsection{Catalysis}

Hydration reactions were performed under argon in NMR tubes capped with septa. Reactions were heated in an aluminium heating block designed to fit 14 NMR tubes.

\section{Reaction of terminal alkynes and hexene with 33}

\section{Substrates:}

Phenylacetylene

4-tert-Butylphenylacetylene

tert-Butylacetylene

4-Ethynyltoluene

(Triisopropylsilyl)acetylene

1-Hexyne

1-Hexene

\section{Conditions:}

$5 \mathrm{~mol} \% 33$ (142 $\mu \mathrm{L}$ acetone/water solution)

$0.1 \mathrm{mmol}$ substrate

16 hours

$100{ }^{\circ} \mathrm{C}$

$0.2 \mathrm{~mL} \mathrm{D}_{2} \mathrm{O} / 0.2 \mathrm{~mL}$ acetone- $\mathrm{d}_{6}$.

GC-MS analysis was required in order to fully analyse the products of these reactions.

(E)-1,4-Bis(triisopropylsilyl)-1-buten-3-yne (38)

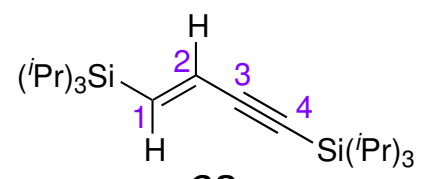

38

Figure 7.1 Numbering scheme for 38 .

NMR data match those reported by Sidera et al. ${ }^{157}{ }^{1} \mathrm{H}$ NMR: $\left(500 \mathrm{MHz}, \mathrm{CDCl}_{3}\right)$ $\delta_{H} 6.36\left(\mathrm{~d},{ }^{3} J_{\mathrm{H}-\mathrm{H}}=19.6 \mathrm{~Hz}, 1 \mathrm{H}, \mathrm{H} 1\right), 6.03\left(\mathrm{~d},{ }^{3} J_{\mathrm{H}-\mathrm{H}}=20.3 \mathrm{~Hz}, 1 \mathrm{H}, \mathrm{H} 2\right), 1.00-1.07$ (s, $\left.42 \mathrm{H}, 6\left(\mathrm{CH}_{3}\right)_{2} \mathrm{CH}\right) \cdot{ }^{13} \mathrm{C}\left\{{ }^{1} \mathrm{H}\right\} \mathrm{NMR}:\left(125 \mathrm{MHz}, \mathrm{CDCl}_{3}\right) \delta_{\mathrm{C}} 141.16(\mathrm{~s}, \mathrm{C} 1), 125.76(\mathrm{~s}$, C2), 107.7 (s, C3), 90.44 (s, C4), $18.75\left(\mathrm{~s}, \mathrm{CH}_{3}\right), 18.68$ (s, $\left.\mathrm{CH}_{3}\right), 11.43$ (s, CH), 10.89 $(\mathrm{s}, \mathrm{CH})$. 


\section{Hydration of Nitriles}

Nitriles were passed through a short plug of alumina before use. Reactions were monitored by ${ }^{1} \mathrm{H}$ NMR spectroscopy and carried out in $\mathrm{D}_{2} \mathrm{O}(0.3 \mathrm{~mL})$. Catalyst solutions were made up in a mixture of acetone and water. Dimethyl sulfone was used as an internal standard as the singlet resonance at $\delta_{H} 3.18 \mathrm{ppm}\left(\mathrm{D}_{2} \mathrm{O}\right)$ did not overlap with any of the resonances of the substrates or product amides.

\section{Acetonitrile}

Acetonitrile $\delta_{H} 2.06\left(\mathrm{~s}, 3 \mathrm{H}, \mathrm{CH}_{3}\right)$; acetamide $1.99\left(\mathrm{~s}, 3 \mathrm{H}, \mathrm{CH}_{3}\right)$.

\section{2-cyanopyridine}

2-cyanopyridine $\delta_{H} 7.71(\mathrm{~m}, 1 \mathrm{H}) ; 2$-pyridinecarboxamide $\delta_{H} 7.58(\mathrm{~m}, 1 \mathrm{H})$.

\section{Propionitrile}

Propionitrile $\delta_{H} 1.25\left(\mathrm{t},{ }^{3} J_{\mathrm{H}-\mathrm{H}}=7.54 \mathrm{~Hz}, 3 \mathrm{H}\right)$; propanamide $\delta_{H} 1.11\left(\mathrm{t},{ }^{3} J_{\mathrm{H}-\mathrm{H}}=7.59\right.$ $\mathrm{Hz}, 3 \mathrm{H})$.

\section{Comparison between ${ }^{2-p y r} \mathrm{PNP}$ and ${ }^{\mathrm{Ph}} \mathrm{PNP}$}

\section{Substrates}

2-cyanopyridine

Propionitrile

Acetonitrile

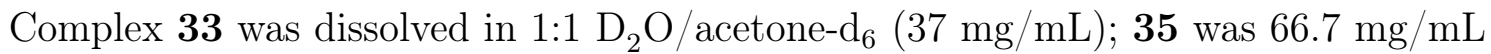
2:1 acetone- $\mathrm{d}_{6} / \mathrm{D}_{2} \mathrm{O}$.

The nitrile substrate $(0.1 \mathrm{mmol})$ in $\mathrm{D}_{2} \mathrm{O}(0.3 \mathrm{~mL})$ was combined with $0.5 \mathrm{~mol} \%$ 33, 35, and pyridine, respectively. The solvent mixtures were adjusted so that the amounts of acetone and water were equivalent $\left(0.355 \mathrm{~mL} \mathrm{D}_{2} \mathrm{O}, 55 \mu \mathrm{L}\right.$ acetone- $\left.\mathrm{d}_{6}\right)$. Reaction were also set up for each substrate with no added catalyst. 


\section{Appendix}

\subsection{Crystallographic Data for $\left[\left({ }^{2-p y r} \mathrm{PNP}\right) \operatorname{Ir}(\operatorname{cod})\right] \mathrm{Cl}$}

(33)

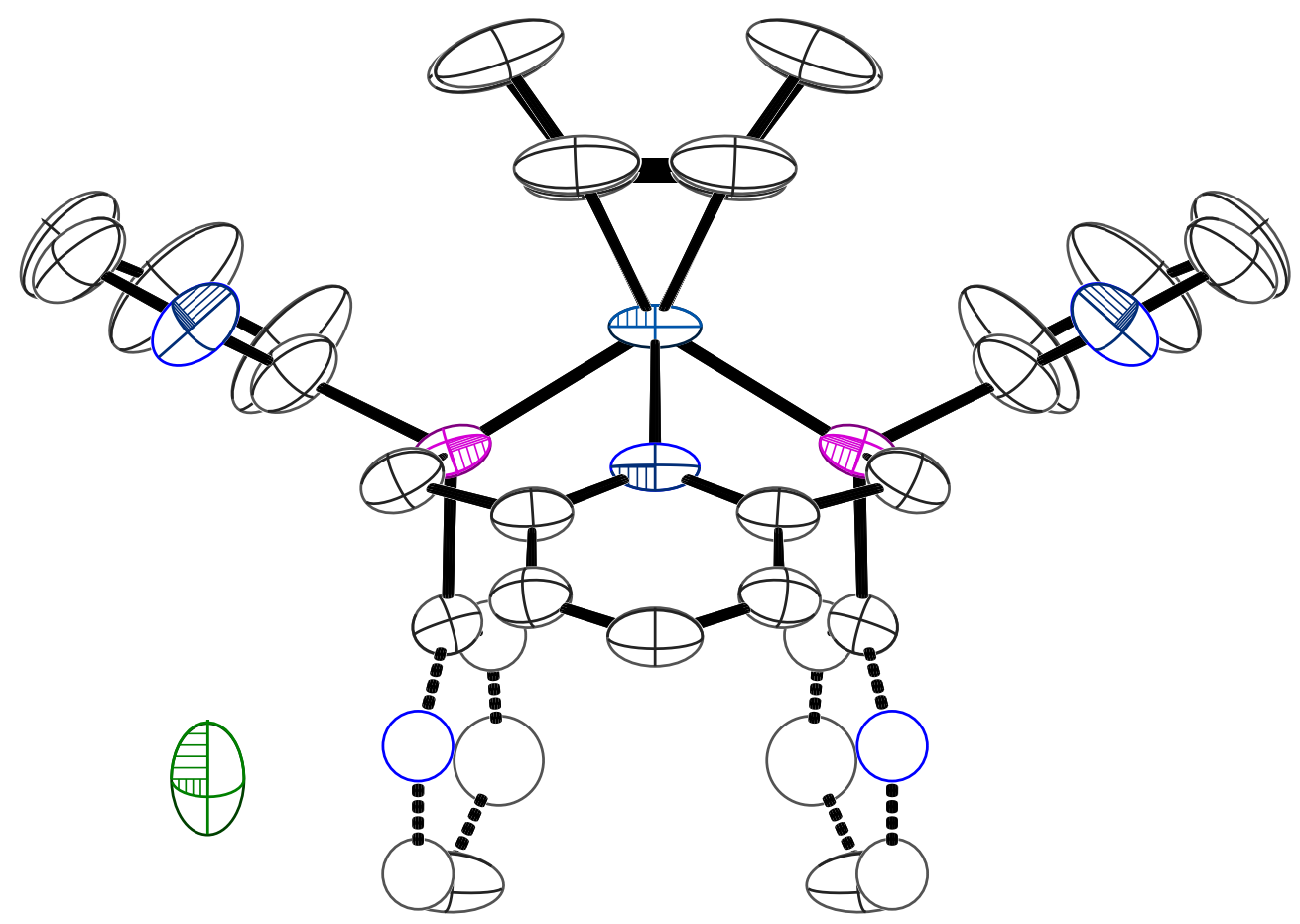

Figure 8.2 X-ray structure of $\left[\left({ }^{2-p y r} \mathrm{PNP}\right) \operatorname{Ir}(\operatorname{cod})\right] \mathrm{Cl}(\mathbf{3 3})$ showing the second part of the disordered pyridyl rings.

One set of pyridyl rings in $\mathbf{3 3}$ is disordered by rotation about the $\mathrm{P}-\mathrm{C}_{\text {pyridyl }}$ bond. The disordered solvent was unable to be modelled successfully, hence the high R-factors displayed in Table 8.1. 
Table 8.1 Crystallographic data of $\left[\left({ }^{2-p y r} \mathrm{PNP}\right) \operatorname{Ir}(\operatorname{cod})\right] \mathrm{Cl}(\mathbf{3 3})$.

\begin{tabular}{|c|c|}
\hline Empirical formula & $\mathrm{C}_{35} \mathrm{H}_{35} \mathrm{~N}_{5} \mathrm{P}_{2} \mathrm{ClIr}$ \\
\hline Formula weight & 813.3 \\
\hline Temperature/K & $120.01(10)$ \\
\hline Crystal system & monoclinic \\
\hline Space group & $\mathrm{C} 2 / m$ \\
\hline $\mathrm{a} / \AA$ & $21.0704(13)$ \\
\hline $\mathrm{b} / \AA$ & $22.6762(14)$ \\
\hline $\mathrm{c} / \AA$ & $11.6280(7)$ \\
\hline$\alpha /{ }^{\circ}$ & 90 \\
\hline$\beta /{ }^{\circ}$ & $125.924(9)$ \\
\hline$\gamma /{ }^{\circ}$ & 90 \\
\hline Volume $/ \AA^{3}$ & $4499.1(7)$ \\
\hline $\mathrm{Z}$ & 4 \\
\hline$\rho_{\text {calc }} \mathrm{g} / \mathrm{cm}^{3}$ & 1.2006 \\
\hline$\mu / \mathrm{mm}^{-1}$ & 3.122 \\
\hline $\mathrm{F}(000)$ & 1604.8 \\
\hline Crystal size $/ \mathrm{mm}^{3}$ & $0.1629 \times 0.0992 \times 0.0463$ \\
\hline Radiation & Mo $\mathrm{K} \alpha(\lambda=0.71073)$ \\
\hline $2 \Theta$ range for data collection $/{ }^{\circ}$ & 5.5 to 54.96 \\
\hline Index ranges & $-27 \leq \mathrm{h} \leq 27,-29 \leq \mathrm{k} \leq 29,-14 \leq \mathrm{l} \leq 15$ \\
\hline Reflections collected & 28619 \\
\hline Independent reflections & $5291\left[\mathrm{R}_{\text {int }}=0.0618, \mathrm{R}_{\text {sigma }}=0.0377\right]$ \\
\hline Data/restraints/parameters & $5291 / 0 / 200$ \\
\hline Goodness-of-fit on $\mathrm{F}^{2}$ & 1.771 \\
\hline Final $\mathrm{R}$ indexes $[\mathrm{I}>=2 \sigma(\mathrm{I})]$ & $\mathrm{R}_{1}=0.1293, \mathrm{wR}_{2}=0.3728$ \\
\hline Final $R$ indexes [all data] & $\mathrm{R}_{1}=0.1431, \mathrm{wR}_{2}=0.3936$ \\
\hline Largest diff. peak/hole / e $\AA^{-3}$ & $6.27 /-2.50$ \\
\hline
\end{tabular}




\subsection{NMR Spectra of Selected Compounds}

\section{Ligand Synthesis}

${ }^{2-p y r} \mathrm{PNP}$

${ }^{2-p y r} \mathrm{PNP}^{-\mathrm{O}_{2}}(\mathbf{2 5})$

${ }^{t} \mathrm{Bu} \mathrm{PNP}\left(\mathrm{BH}_{3}\right)$ (8)

Vinyl region of the ${ }^{1} \mathrm{H}$ NMR spectrum from the synthesis of ${ }^{\mathrm{Vi}} \mathrm{PNP}$

\section{Rhodium}

${ }^{2-\text { pyr }} \mathrm{PNP}+\left[\mathrm{Rh}(\text { acetone })_{2}(\text { coe })_{2}\right]$ in acetone

${ }^{2-\text { pyr }} \mathrm{PNP}+\left[\mathrm{Rh}(\text { coe })_{2}(\mu \text {-Cl) }]_{2}\right.$ in toluene

${ }^{t} \mathrm{Bu} \mathrm{PNP}+\left[\mathrm{Rh}(\text { coe })_{2}(\mu-\mathrm{Cl})\right]_{2}$

$\mathrm{AgBF}_{4}+\left[\left({ }^{t} \mathrm{Bu} \mathrm{PNP}\right) \mathrm{RhCl}\right]$

\section{Iridium}

Full set of $\left[\left({ }^{2-p y r} \mathrm{PNP}\right) \operatorname{Ir}(\mathrm{cod})\right] \mathrm{Cl}(\mathbf{3 3})$

${ }^{2-\text { pyr }} \mathrm{PNP}+[\operatorname{Ir}(\operatorname{cod})(\mu \text {-OMe })]_{2}$

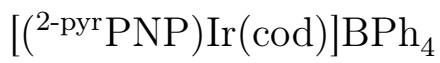

$\left[\left({ }^{2-p y r} \mathrm{PNP}\right) \operatorname{Ir}(\mathrm{H}){ }_{2} \mathrm{Cl}\right](\mathbf{3 7})$

Other NMR data are available on request. 


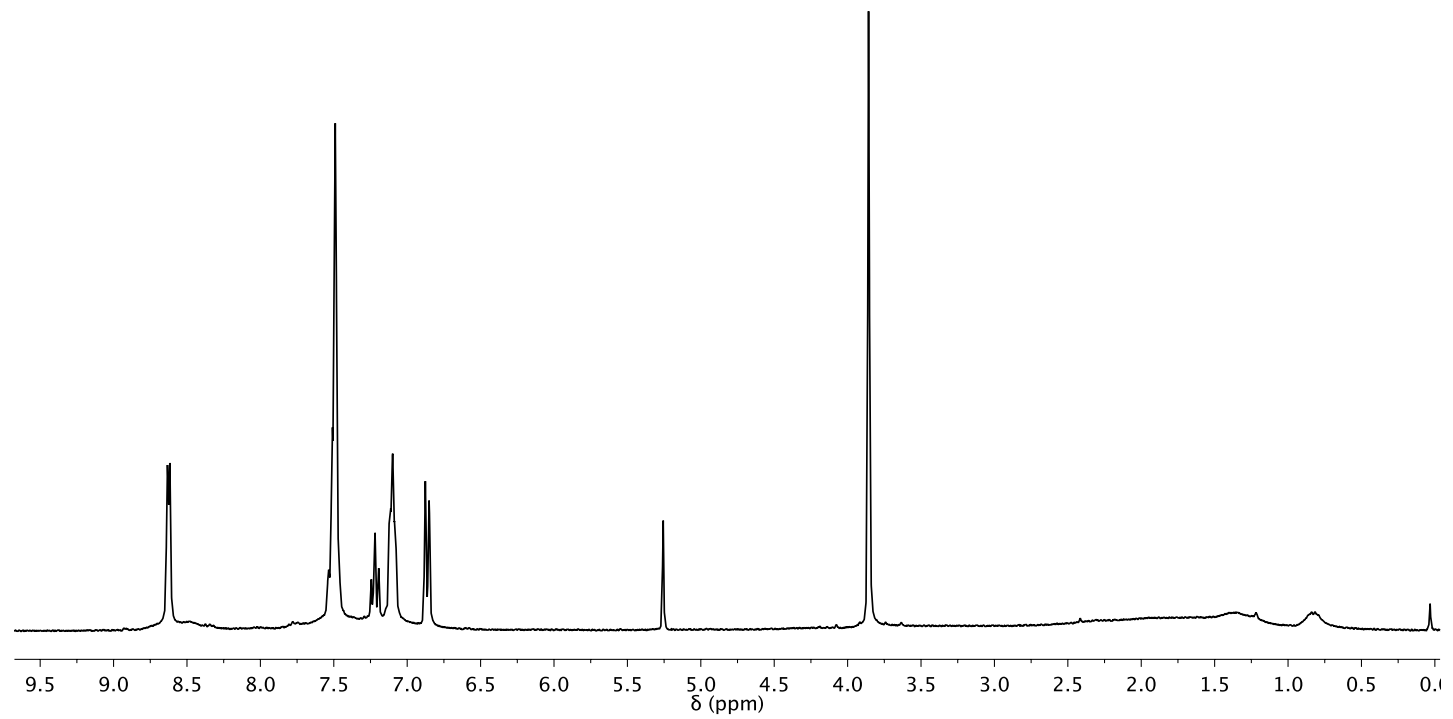

Figure 8.3 ${ }^{1} \mathrm{H}$ NMR spectrum of ${ }^{2-p y r} \mathrm{PNP} . \mathrm{CDCl}_{3}, 300 \mathrm{MHz}$. Dichloromethane at $\delta_{H} 5.30 \mathrm{ppm}$. 


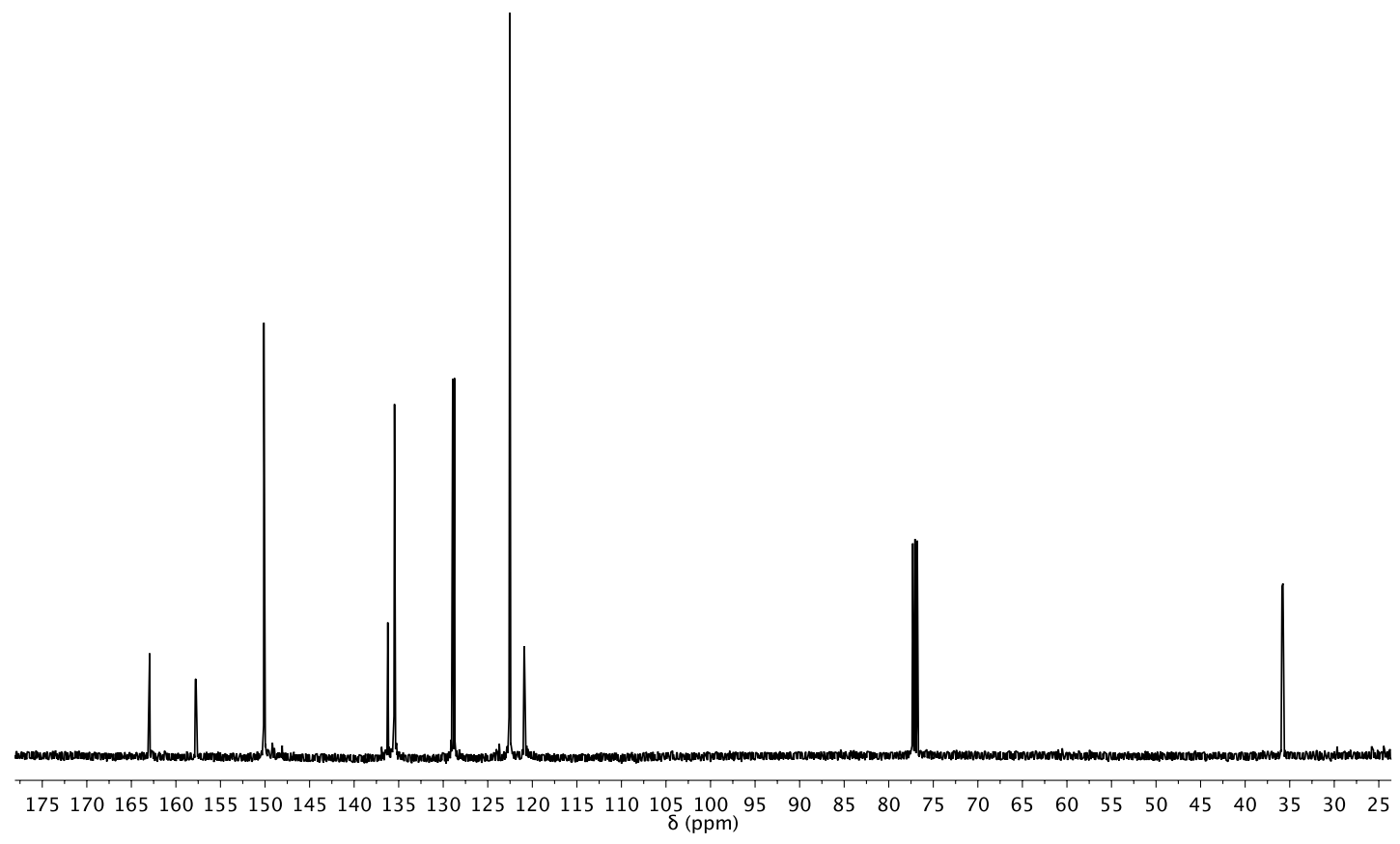

Figure 8.4 ${ }^{13} \mathrm{C}\left\{{ }^{1} \mathrm{H}\right\}$ NMR spectrum of ${ }^{2-p y r} \mathrm{PNP} . \mathrm{CDCl}_{3}, 125 \mathrm{MHz}$.

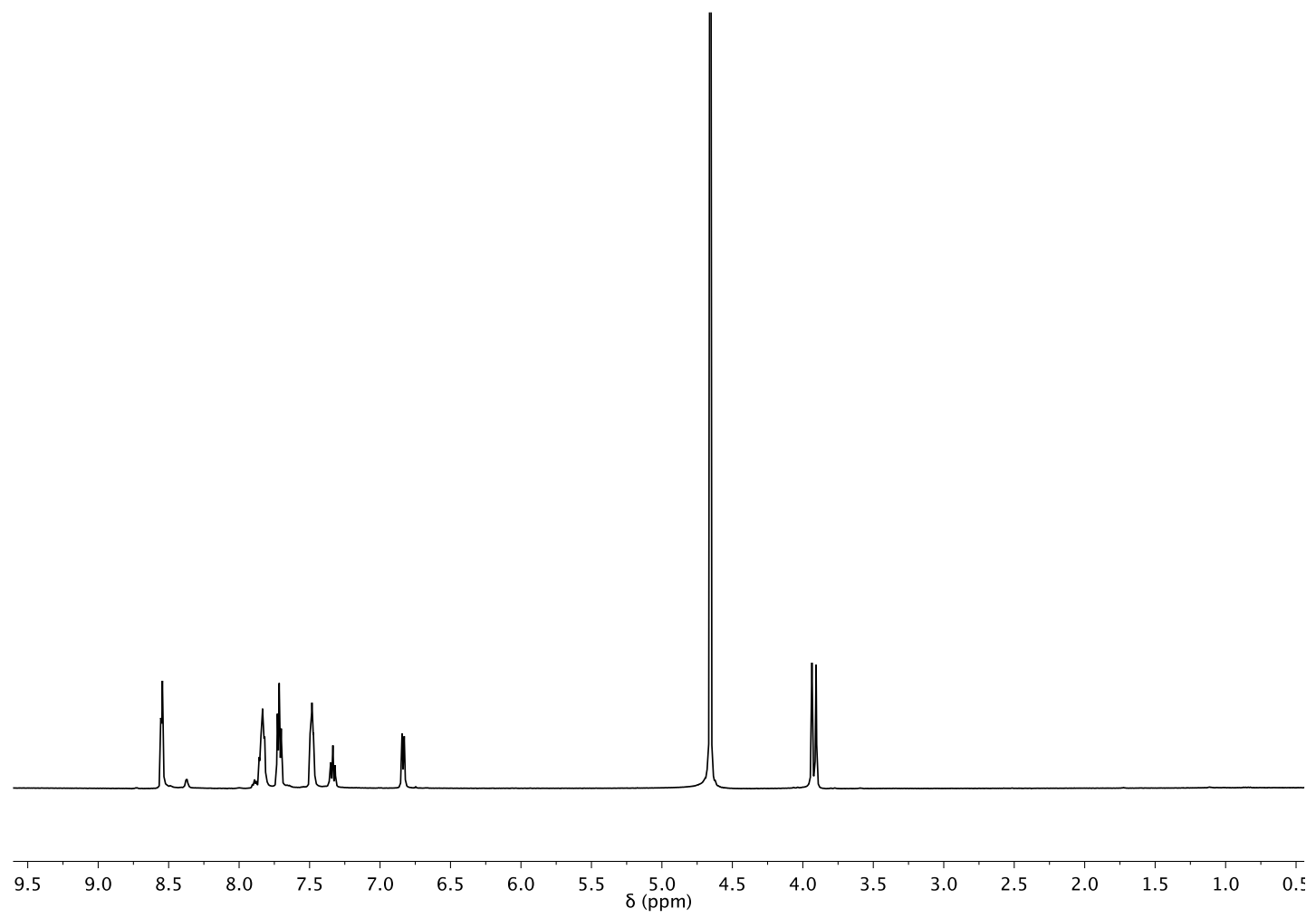

Figure 8.5 ${ }^{1} \mathrm{H}$ NMR spectrum of ${ }^{2-p y r} \mathrm{PNP}_{-} \mathrm{O}_{2}(\mathbf{2 5}) . \mathrm{D}_{2} \mathrm{O}, 500 \mathrm{MHz}$. 


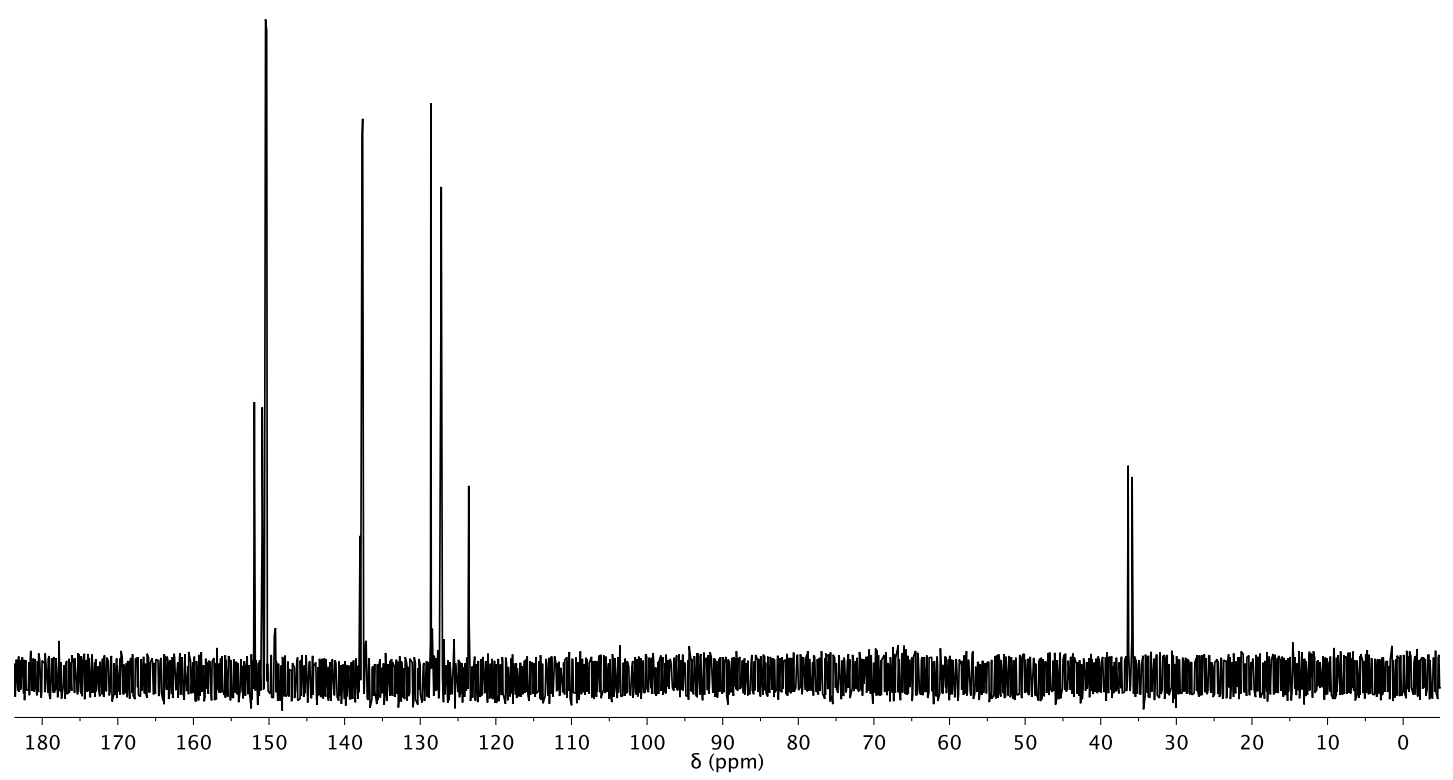

Figure 8.6 ${ }^{13} \mathrm{C}\left\{{ }^{1} \mathrm{H}\right\}$ NMR spectrum of ${ }^{2-p y r} \mathrm{PNP}_{-} \mathrm{O}_{2}(\mathbf{2 5}) . \mathrm{D}_{2} \mathrm{O}, 125 \mathrm{MHz}$.

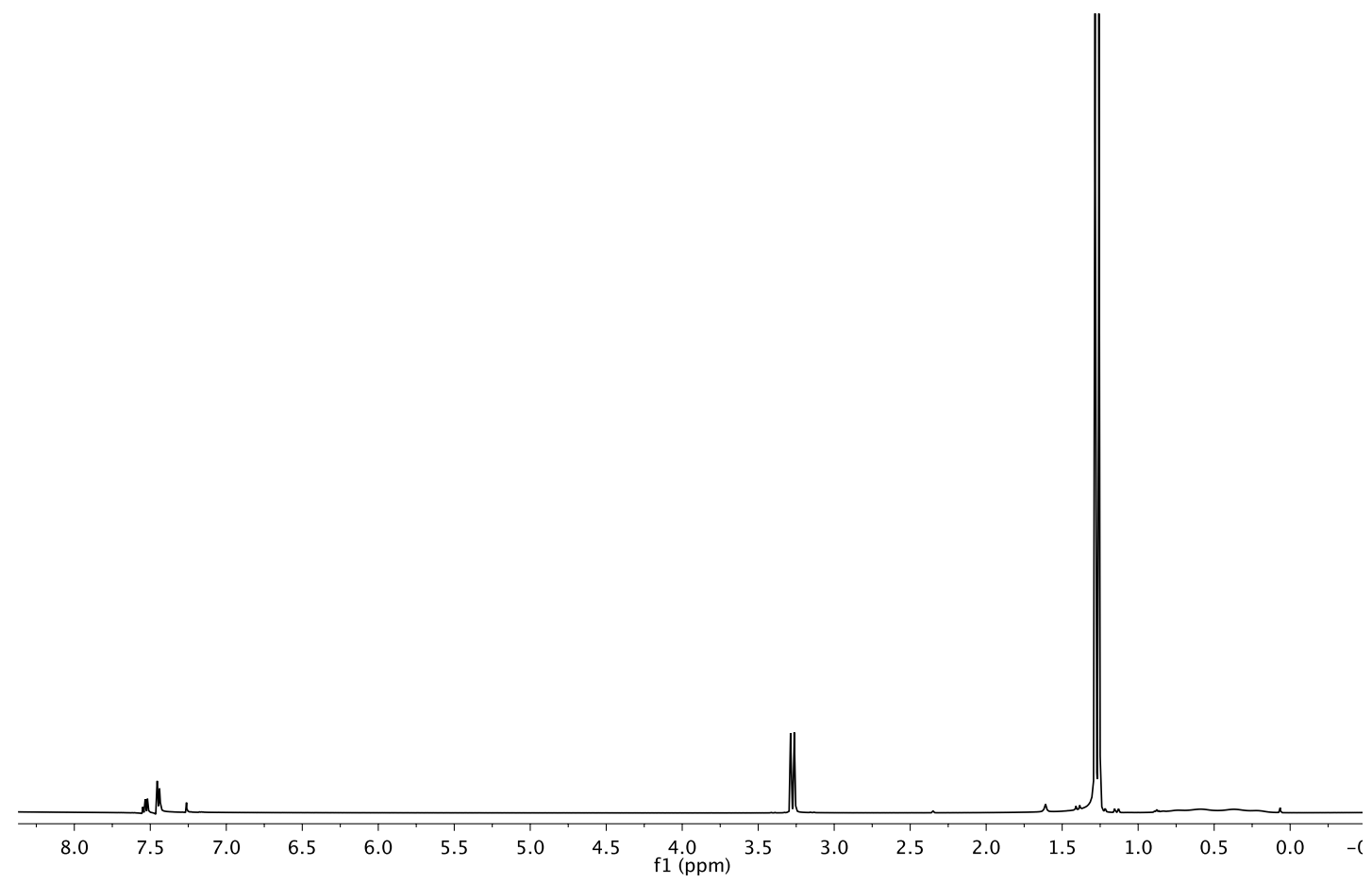

Figure 8.7 ${ }^{1} \mathrm{H}$ NMR spectrum of ${ }^{t} \mathrm{Bu}^{\mathrm{PNP}}-\mathrm{BH}_{3}$ (8). $\mathrm{CDCl}_{3}, 500 \mathrm{MHz}$. 


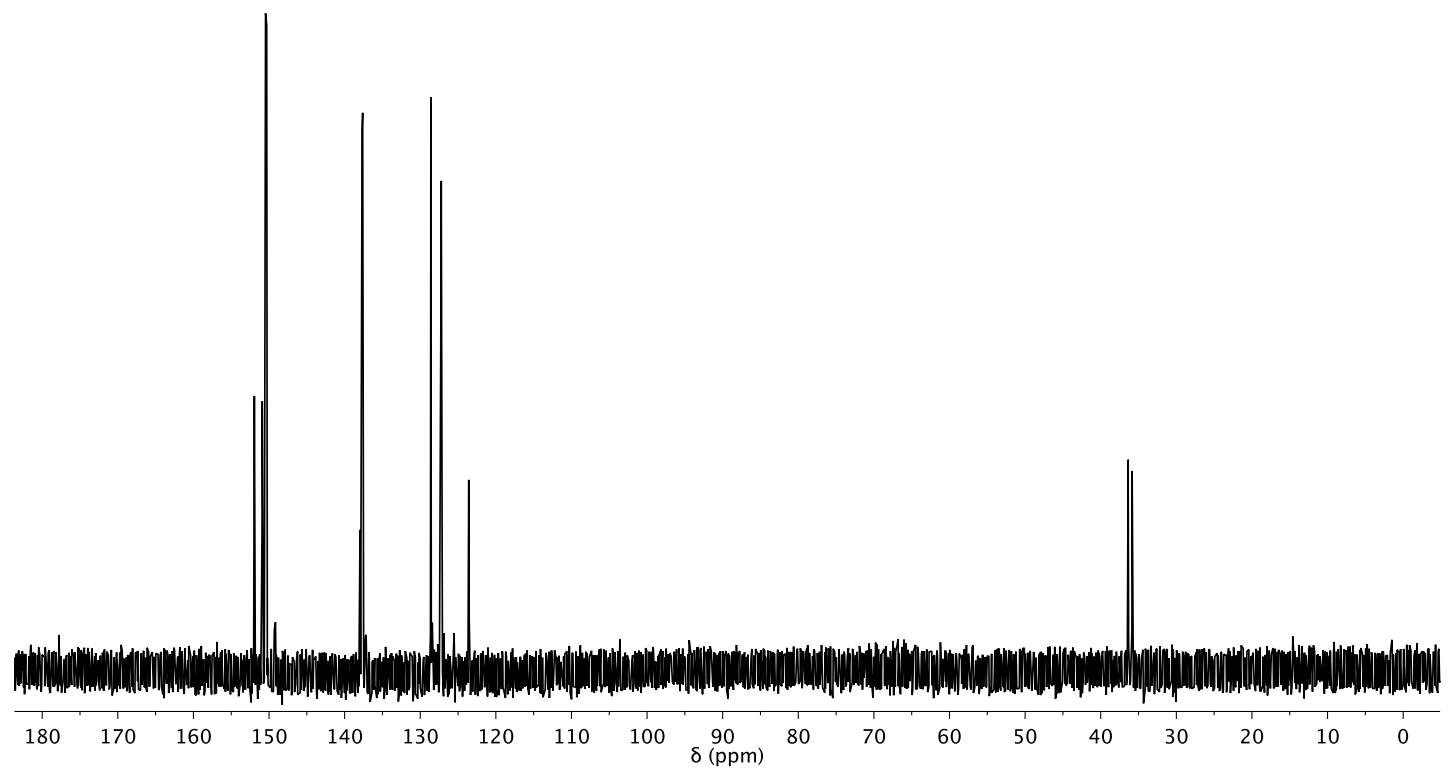

Figure 8.8 ${ }^{13} \mathrm{C}\left\{{ }^{1} \mathrm{H}\right\}$ NMR spectrum of ${ }^{t} \mathrm{Bu}^{\mathrm{PNP}-\mathrm{BH}} 3$ (8). $\mathrm{CDCl}_{3}, 125 \mathrm{MHz}$.

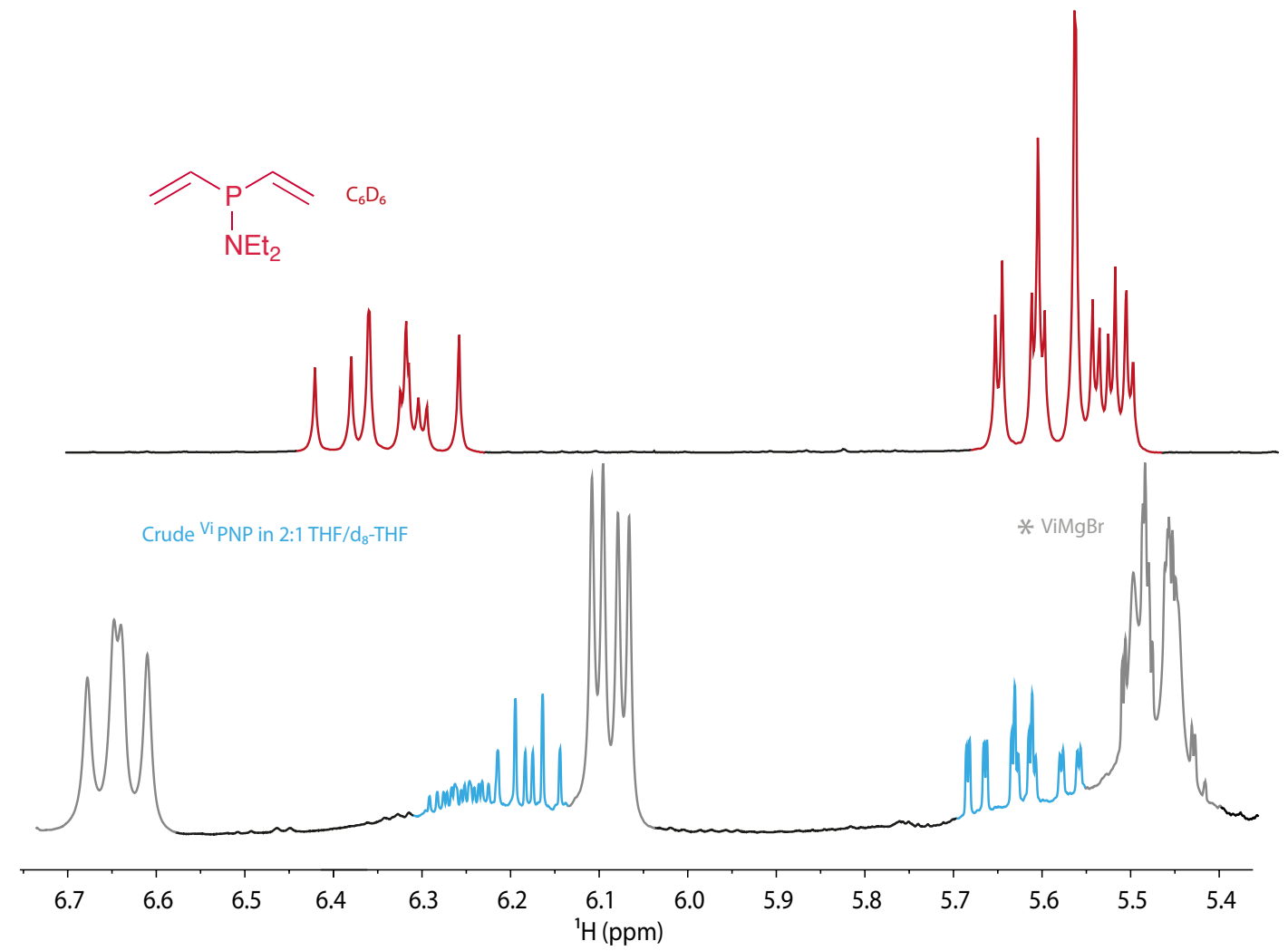

Figure 8.9 The P-Vi groups have distinctive ${ }^{1} \mathrm{H}$ NMR signals. 


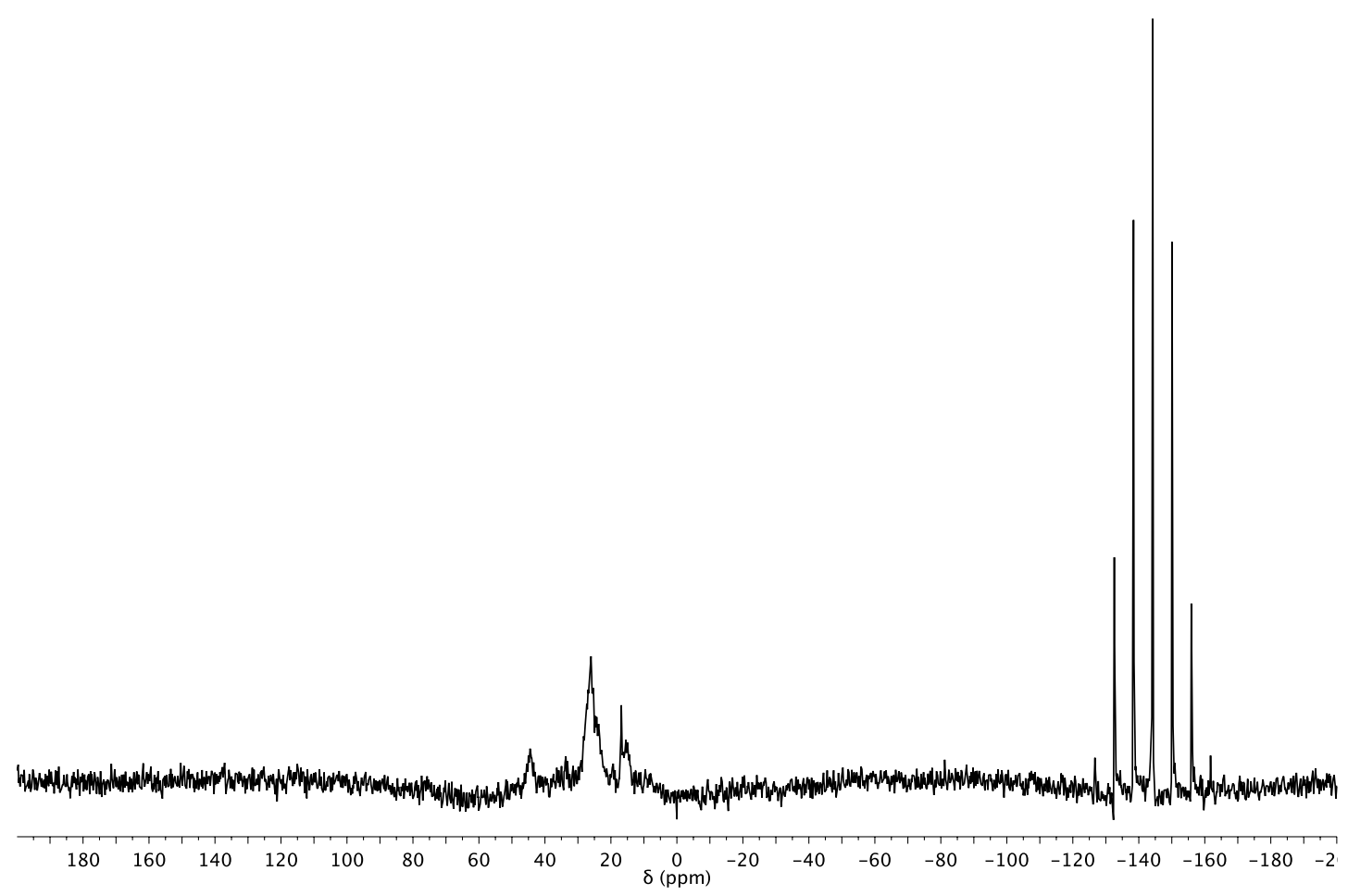

Figure 8.10 ${ }^{31} \mathrm{P}\left\{{ }^{1} \mathrm{H}\right\}$ NMR spectrum of ${ }^{2-\text { pyr }} \mathrm{PNP}+\left[\mathrm{Rh}(\text { acetone })_{2}(\text { coe })_{2}\right]$, at $=0.1$ s. Acetone- $\mathrm{d}_{6}, 121 \mathrm{MHz}$.

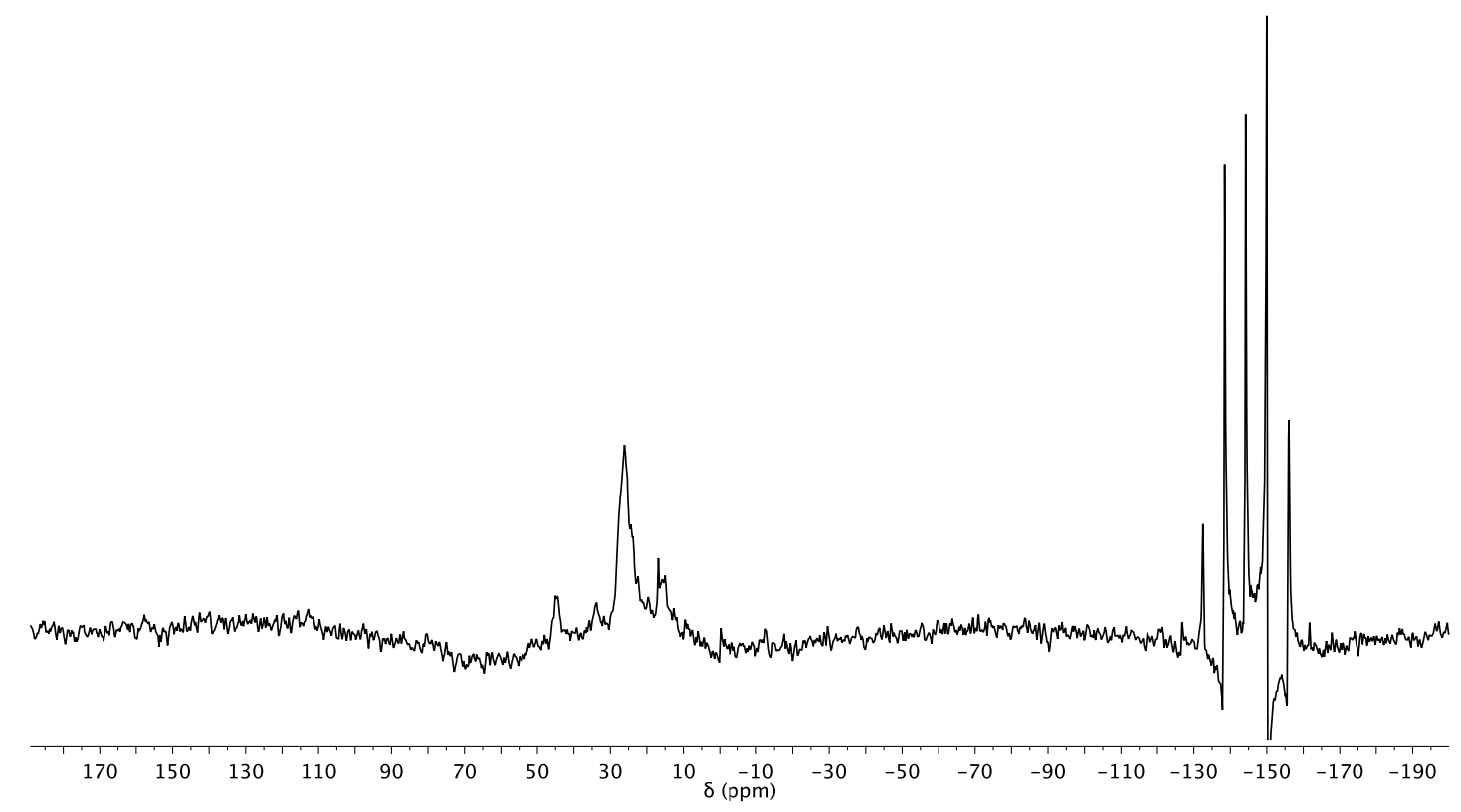

Figure 8.11 ${ }^{31} \mathrm{P}\left\{{ }^{1} \mathrm{H}\right\}$ NMR spectrum of ${ }^{2-p y r} \mathrm{PNP}+\left[\mathrm{Rh}(\text { acetone })_{2}(\mathrm{coe})_{2}\right]$, at $=0.025 \mathrm{~s}$. Acetone- $\mathrm{d}_{6}, 121 \mathrm{MHz}$. 


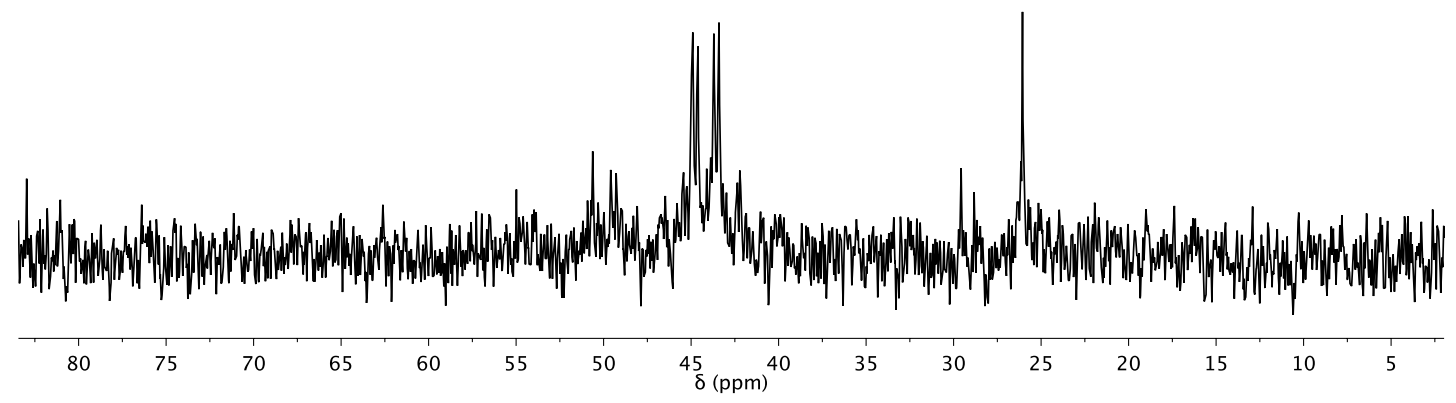

Figure 8.12 ${ }^{31} \mathrm{P}\left\{{ }^{1} \mathrm{H}\right\}$ NMR spectrum of ${ }^{2-\text { pyr }} \mathrm{PNP}+\left[\mathrm{Rh}(\text { coe })_{2}(\mu-\mathrm{Cl})\right]_{2}$ in toluene, at $=1$ s. $\mathrm{CD}_{2} \mathrm{Cl}_{2}, 121 \mathrm{MHz} . \delta_{P} 26.3{ }^{2-\mathrm{pyr}} \mathrm{PNP}-\mathrm{O}_{2}$.

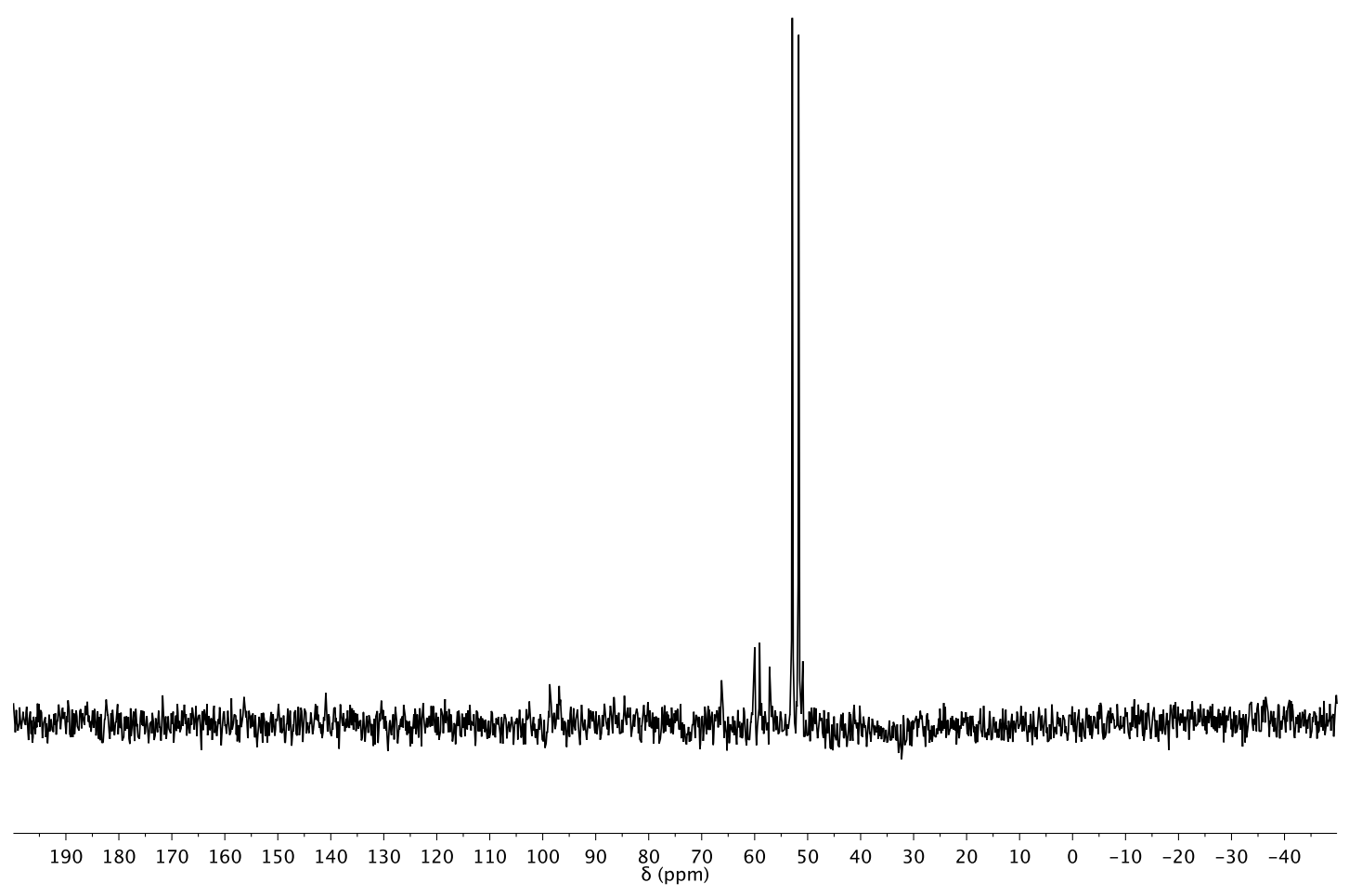

Figure 8.13 ${ }^{31} \mathrm{P}\left\{{ }^{1} \mathrm{H}\right\}$ NMR spectrum of ${ }^{t} \mathrm{Bu} P N P+\left[\mathrm{Rh}(\text { coe })_{2}(\mu-\mathrm{Cl})\right]_{2}$, at $=1 \mathrm{~s}$. $\mathrm{C}_{6} \mathrm{D}_{6}, 121 \mathrm{MHz}$. 


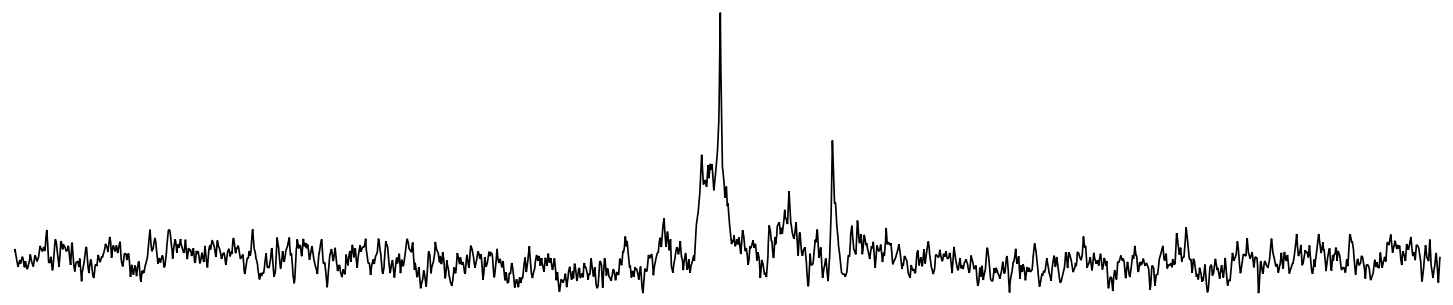

$\begin{array}{llllllllllllllllllllll}190 & 180 & 170 & 160 & 150 & 140 & 130 & 120 & 110 & 100 & \underset{\substack{90 \\(\mathrm{ppm})}}{80} & 70 & 60 & 50 & 40 & 30 & 20 & 10 & 0 & -10 & -20\end{array}$

Figure 8.14 ${ }^{31} \mathrm{P}\left\{{ }^{1} \mathrm{H}\right\}$ NMR spectrum of $\left[\left({ }^{\mathrm{t}}{ }^{\mathrm{PuNP}}\right) \mathrm{RhCl}\right]+\mathrm{AgBF}_{4}$, at $=0.05 \mathrm{~s}$. Acetone- $\mathrm{d}_{6}, 121 \mathrm{MHz}$.

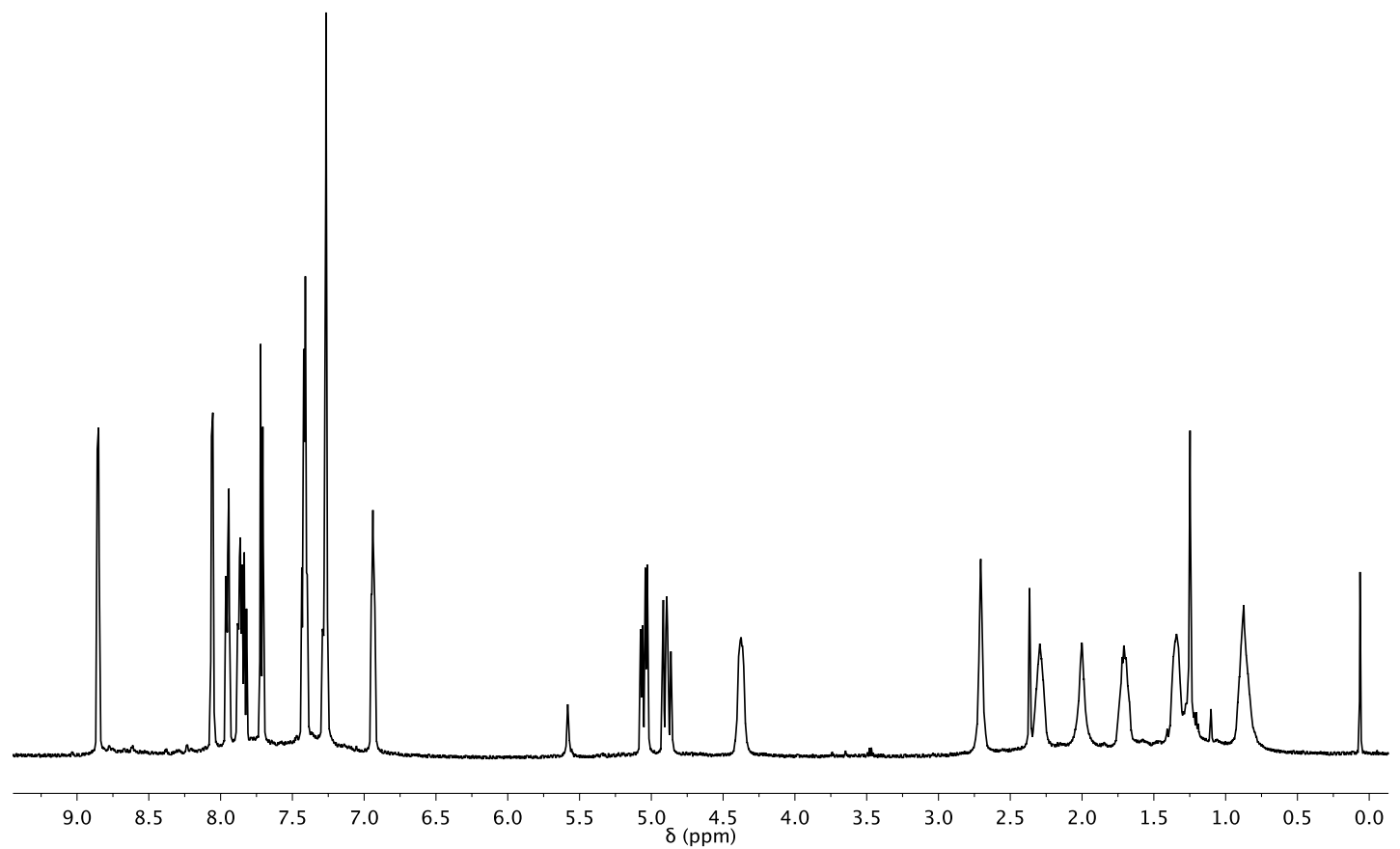

Figure 8.15 ${ }^{1} \mathrm{H}$ NMR spectrum of $\left[\left({ }^{2-p y r} \mathrm{PNP}\right) \operatorname{Ir}(\operatorname{cod})\right] \mathrm{Cl}(33) . \mathrm{CDCl}_{3}, 500 \mathrm{MHz}$. Impurities: cod (5.5 and $2.4 \mathrm{ppm})$, paraffin oil impurity (1.26 and $0.88 \mathrm{ppm})$, and silicon grease $(0.07 \mathrm{ppm})$. 


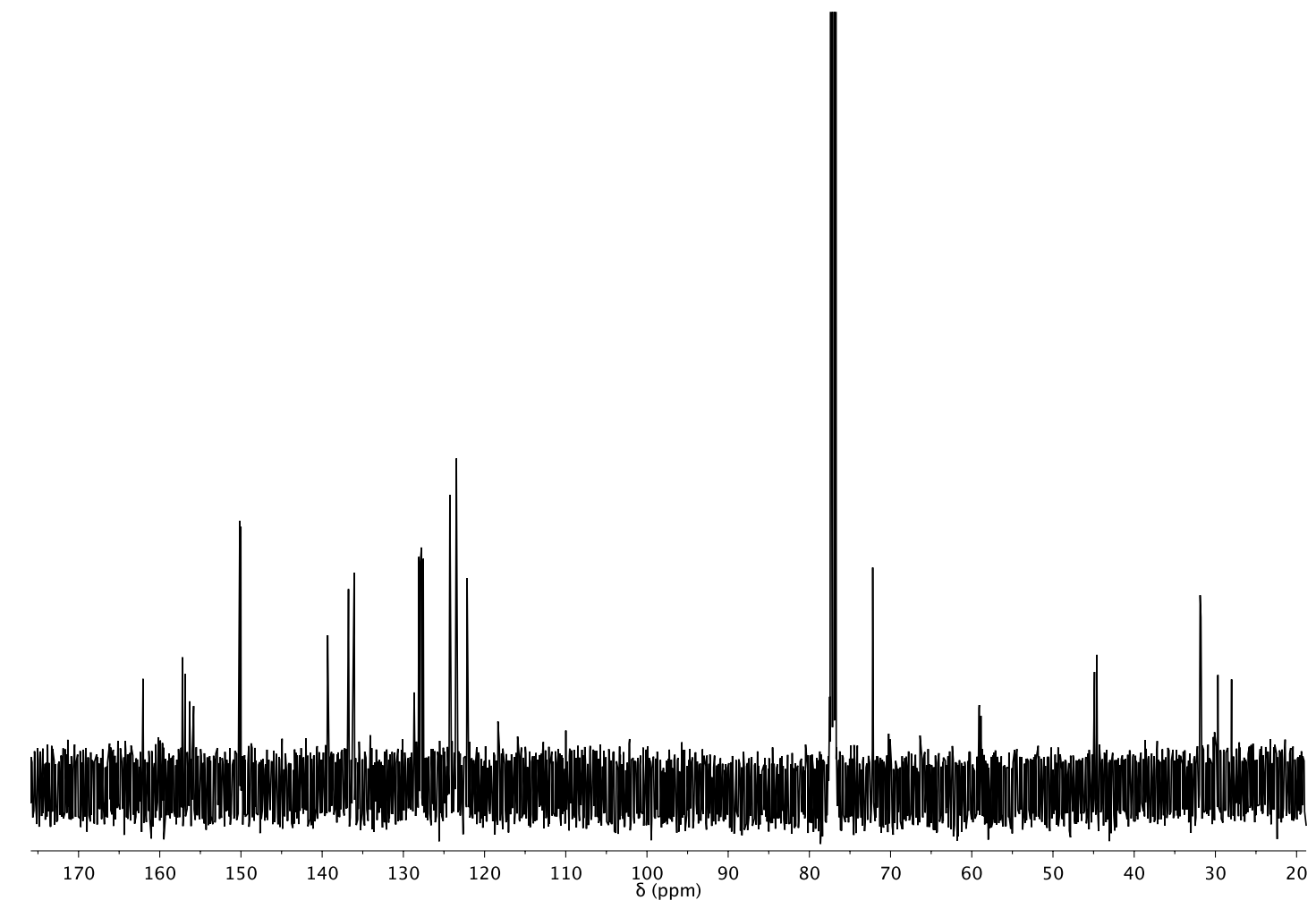

Figure 8.16 ${ }^{13} \mathrm{C}\left\{{ }^{1} \mathrm{H}\right\}$ NMR spectrum of $\left[\left({ }^{2-p y r} \mathrm{PNP}\right) \operatorname{Ir}(\operatorname{cod})\right] \mathrm{Cl}(\mathbf{3 3}) . \mathrm{CDCl}_{3}, 125$ MHz. Impurities: cod (128 and 28 ppm), paraffin oil impurity $(29.7 \mathrm{ppm})$, and silicon grease (1.19 ppm).

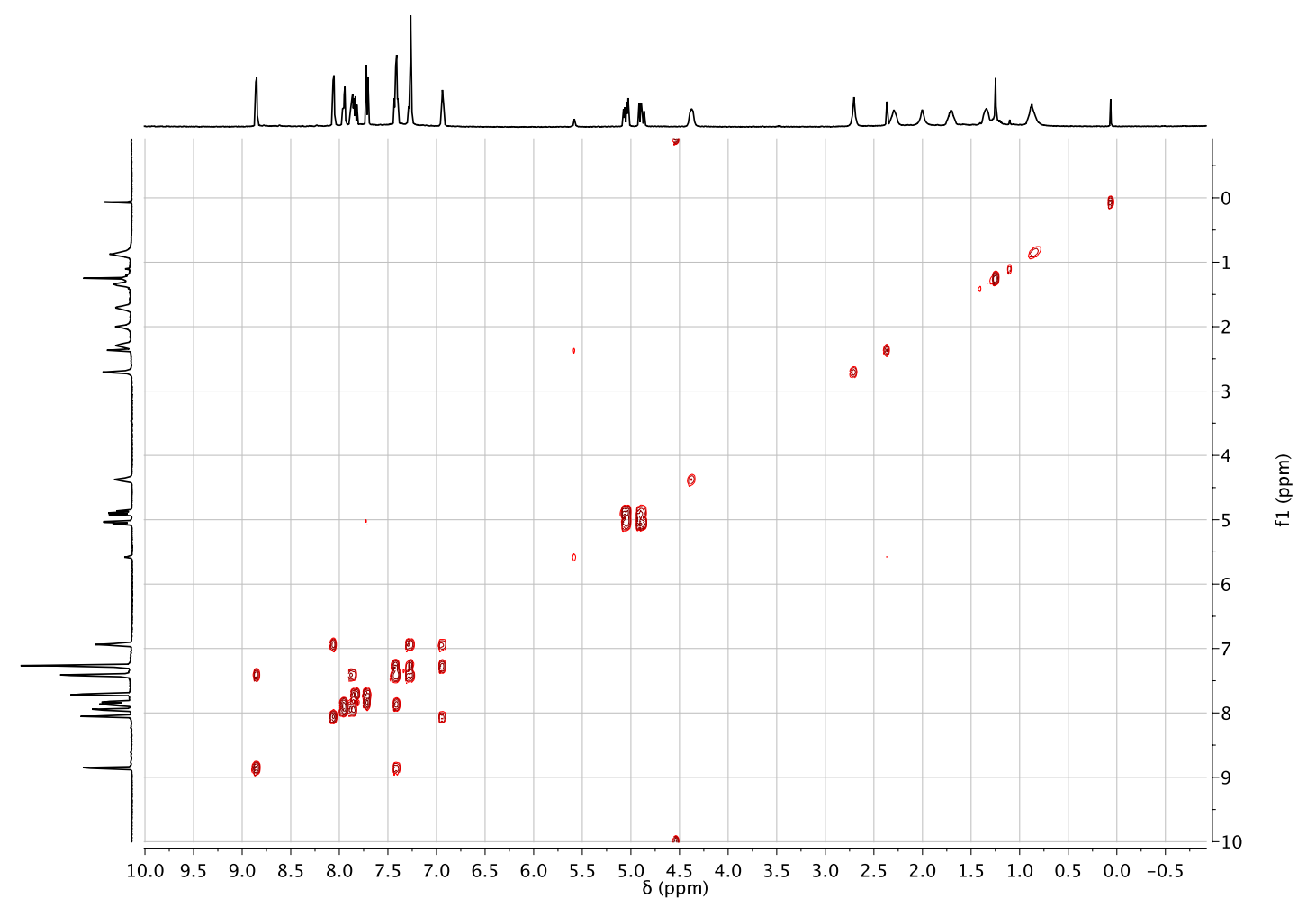

Figure 8.17 COSY spectrum of $\left[\left({ }^{2-p y r} \mathrm{PNP}\right) \operatorname{Ir}(\operatorname{cod})\right] \mathrm{Cl}(\mathbf{3 3}) . \mathrm{CDCl}_{3}, 500 \mathrm{MHz}$. 


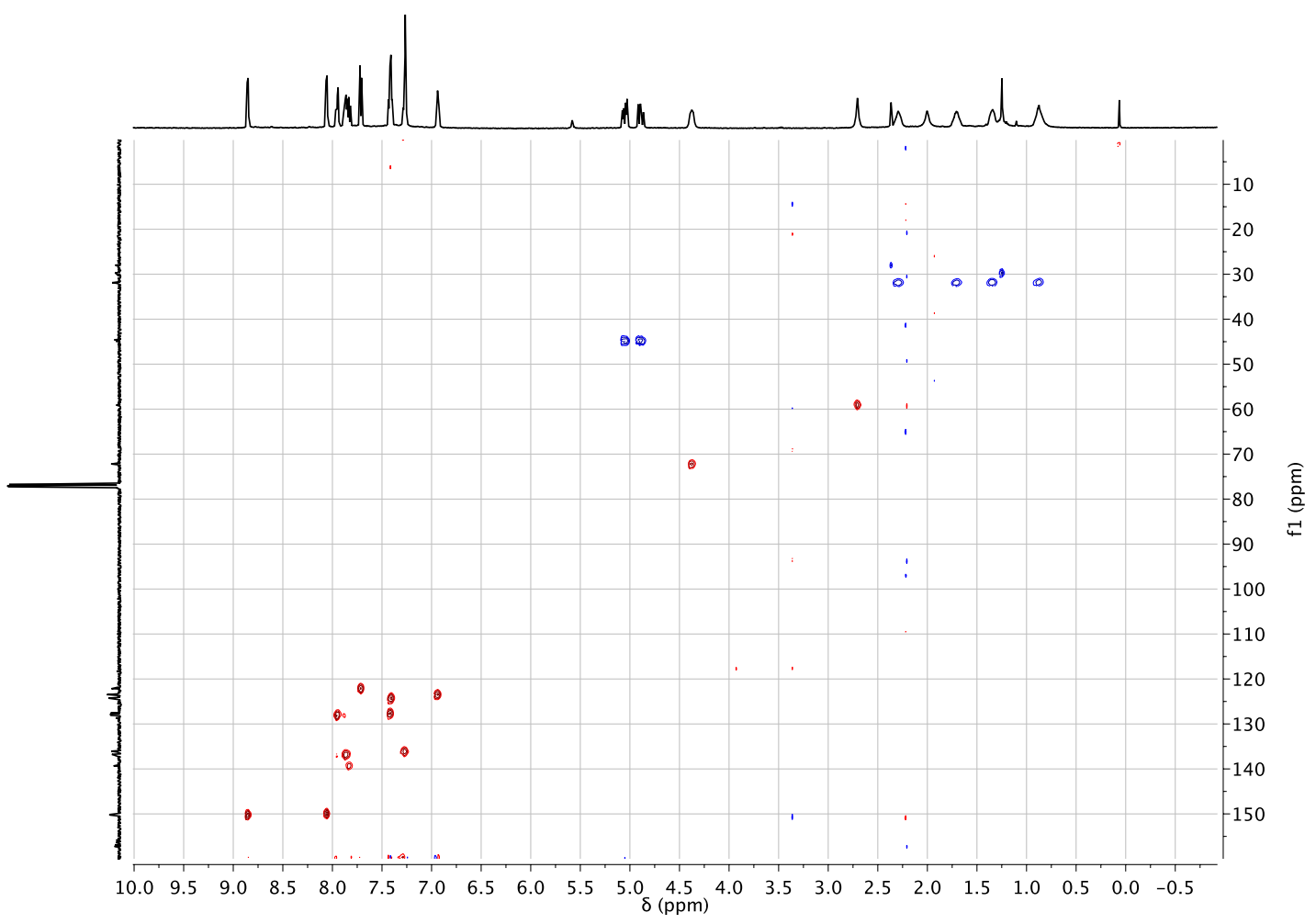

Figure 8.18 HSQC spectrum of $\left[\left({ }^{2-p y r} \mathrm{PNP}\right) \operatorname{Ir}(\operatorname{cod})\right] \mathrm{Cl}(\mathbf{3 3}) . \mathrm{CDCl}_{3}, 500 \mathrm{MHz}$. 


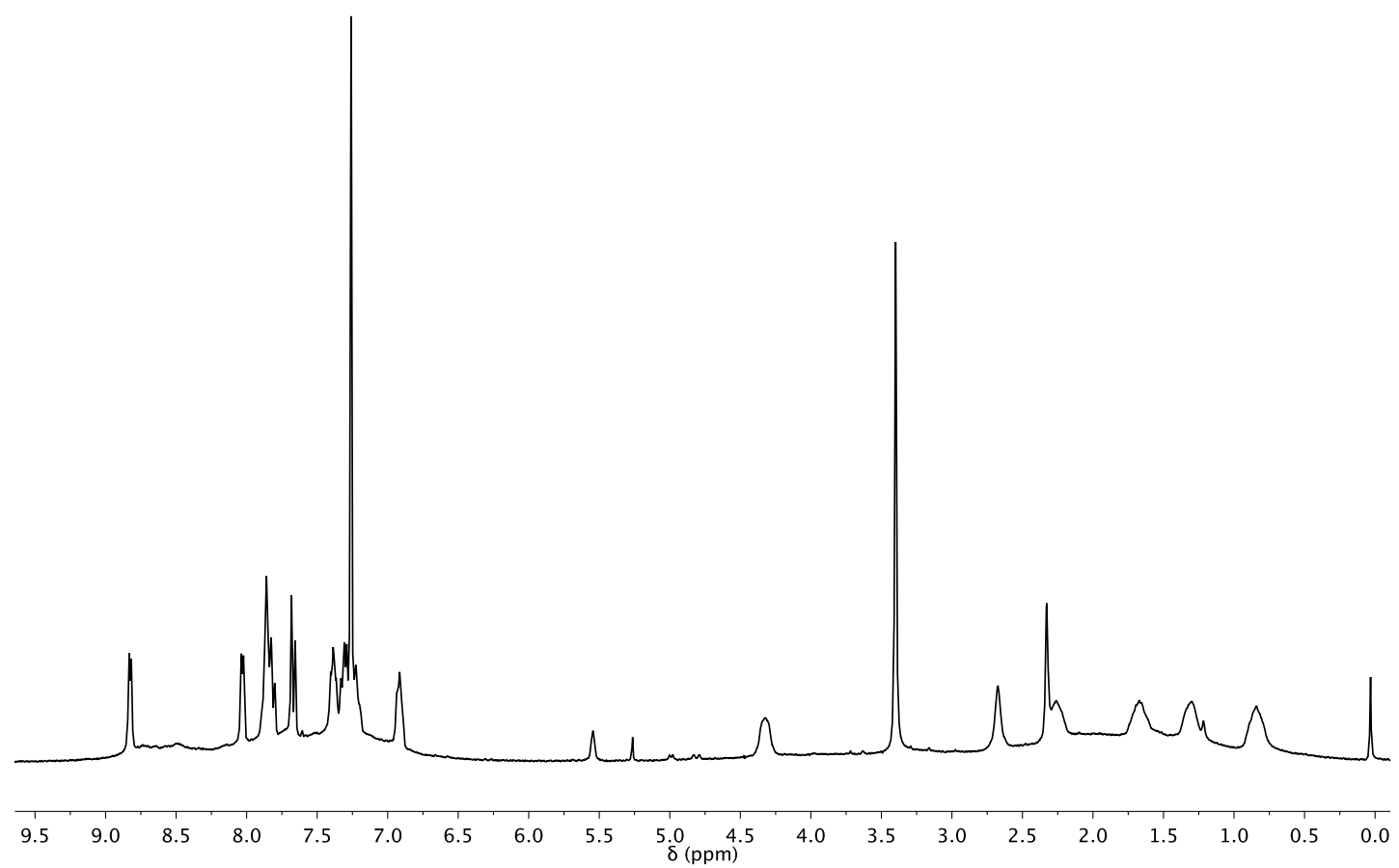

Figure 8.19 ${ }^{1} \mathrm{H}$ NMR spectrum of ${ }^{2-p y r} \mathrm{PNP}+[\operatorname{Ir}(\operatorname{cod})(\mu-\mathrm{OMe})]_{2} \cdot \mathrm{CDCl}_{3}, 500$ $\mathrm{MHz}$. Impurities: cod (5.5 and $2.4 \mathrm{ppm})$, and silicon grease (0.07 ppm). 


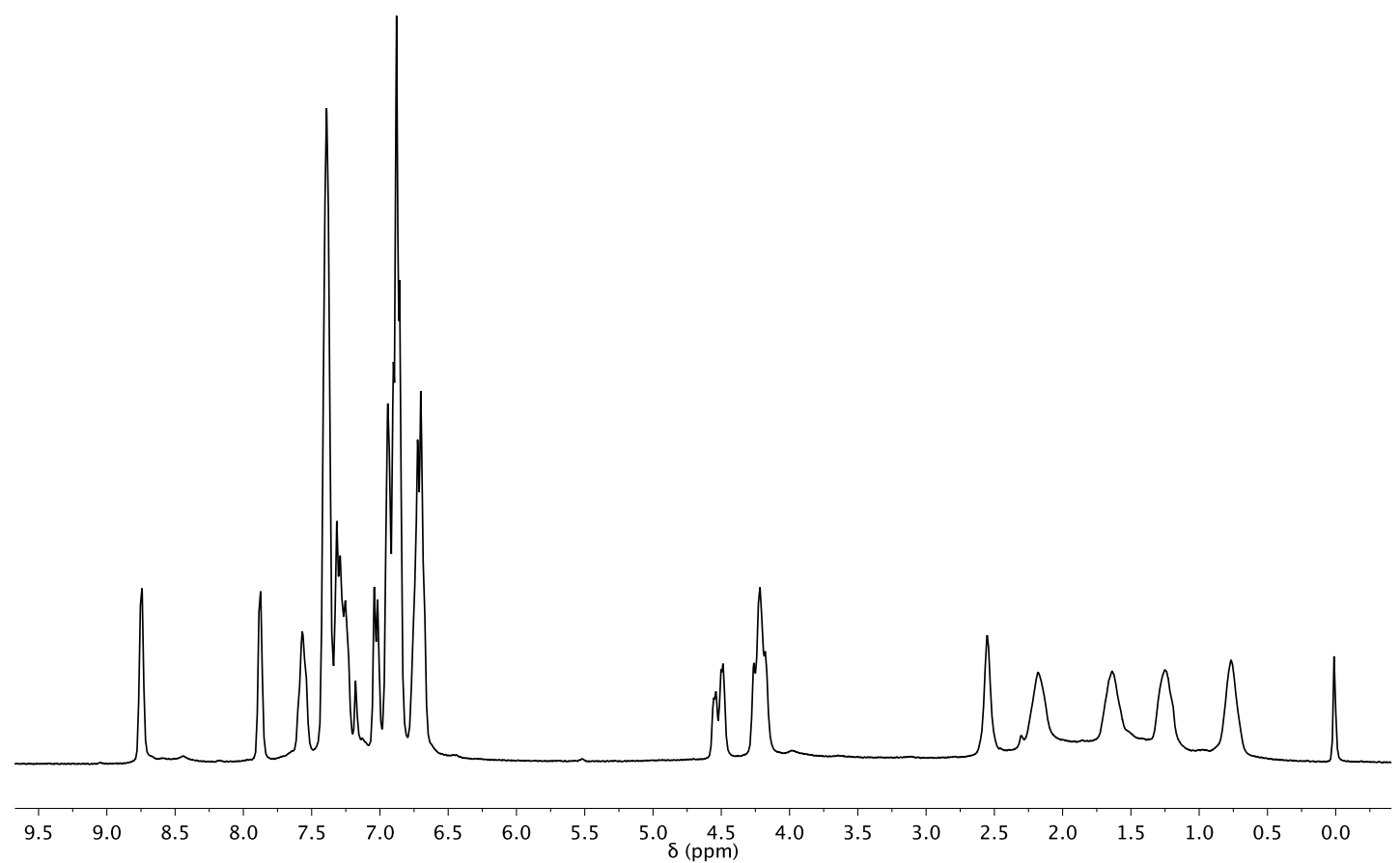

Figure 8.20 ${ }^{1} \mathrm{H}$ NMR spectrum of $\left[\left({ }^{2-p y r} \mathrm{PNP}\right) \operatorname{Ir}(\mathrm{cod})\right] \mathrm{BPh}_{4} \cdot \mathrm{CDCl}_{3}, 300 \mathrm{MHz}$. Impurities: silicon grease (0.07 ppm).

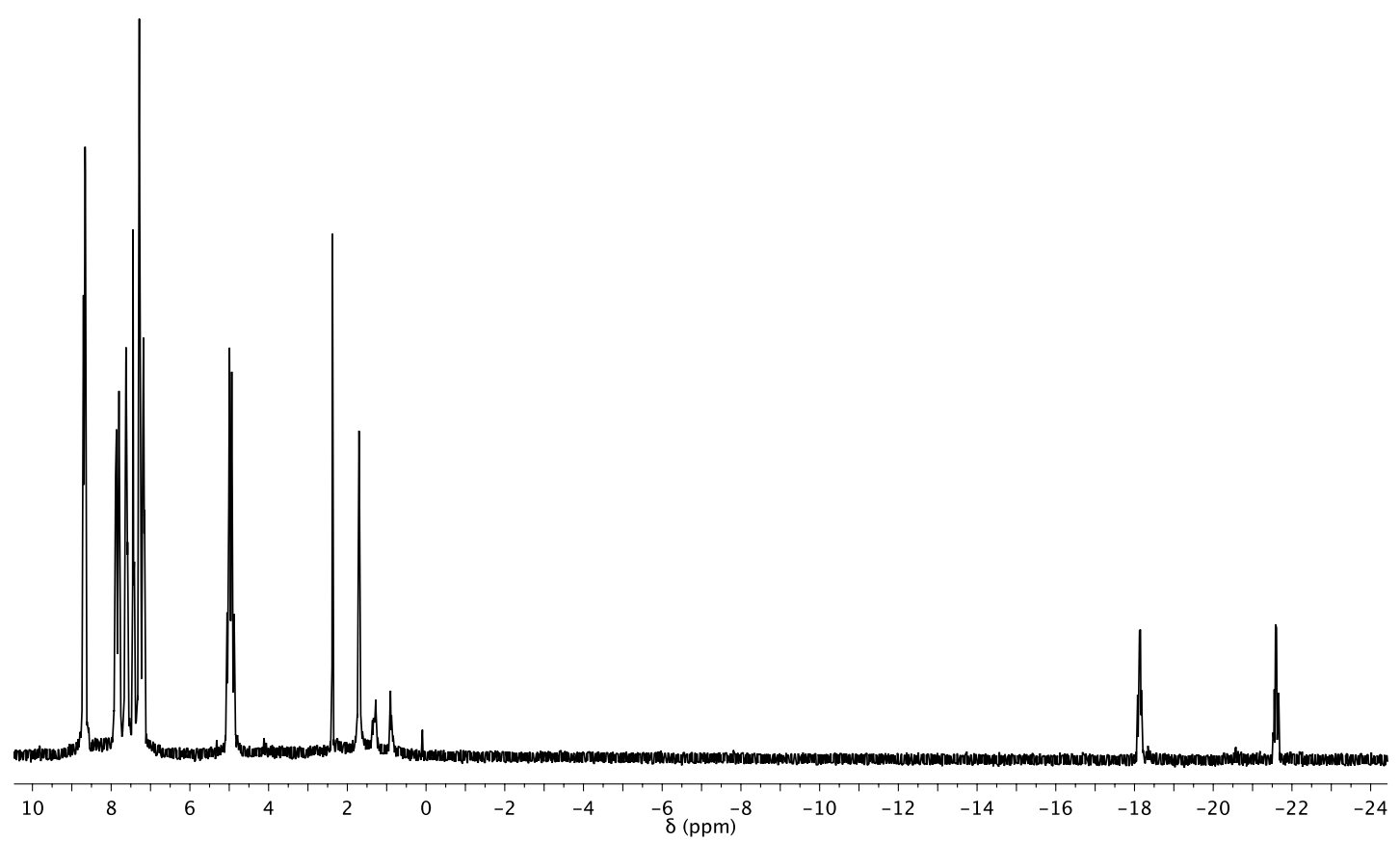

Figure 8.21 ${ }^{1} \mathrm{H}$ NMR spectrum of $\left[\left({ }^{2-p y r} \mathrm{PNP}\right) \operatorname{Ir}(\mathrm{H}){ }_{2} \mathrm{Cl}\right](\mathbf{3 7}) . \mathrm{CDCl}_{3}, 300 \mathrm{MHz}$. Impurities: toluene $(2.34,7.15 \mathrm{ppm})$, water $(1.6 \mathrm{ppm})$, and paraffin oil impurity (1.26 and $0.88 \mathrm{ppm})$. 


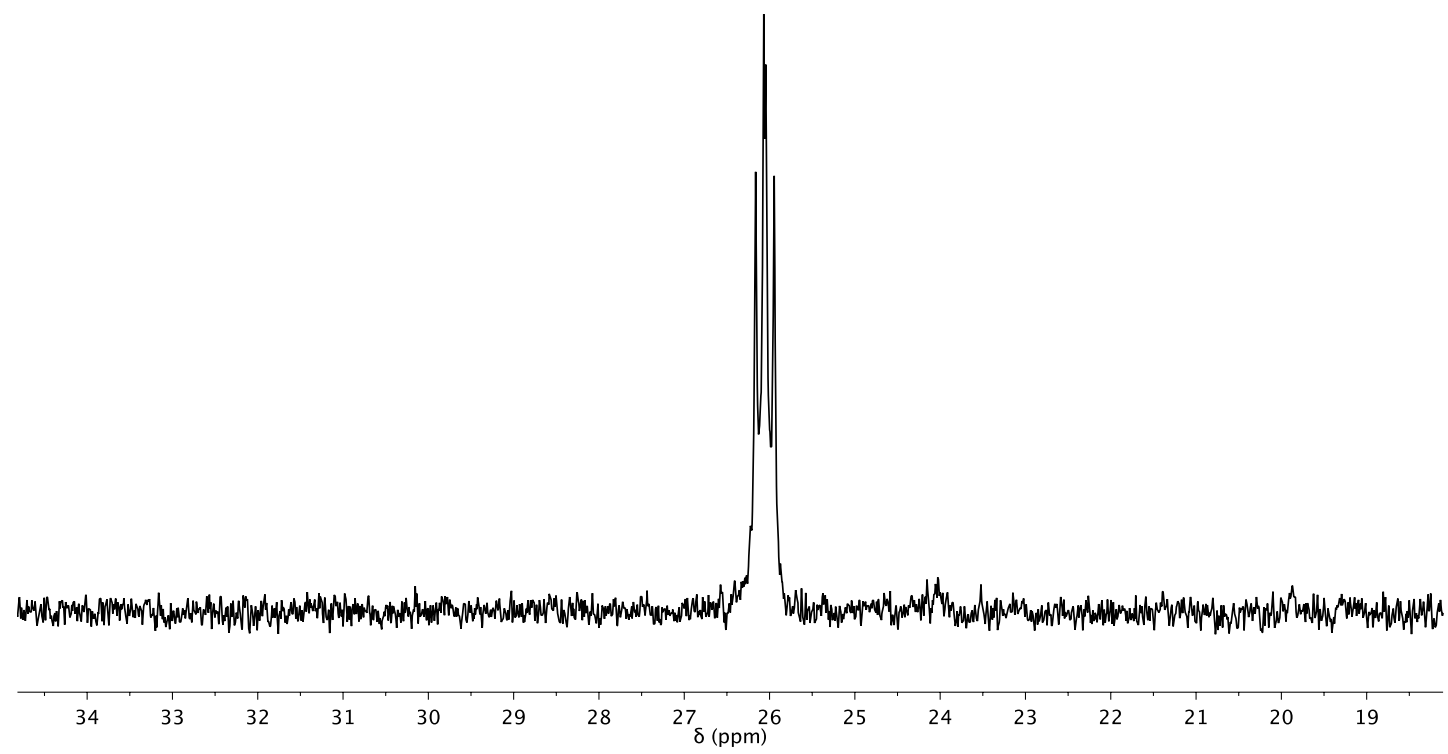

Figure 8.22 ${ }^{31} \mathrm{P}\left\{{ }^{1} \mathrm{H}\right\}$ NMR spectrum of $\left[\left({ }^{2-\mathrm{pyr}} \mathrm{PNP}\right) \operatorname{Ir}(\mathrm{H}){ }_{2} \mathrm{Cl}\right](\mathbf{3 7}) . \mathrm{CDCl}_{3}, 121$ $\mathrm{MHz}$. 


\section{Bibliography}

1. Tundo, P., Perosa, A., Zecchini, F., Eds. Methods and Reagents for Green Chemistry; Wiley-Interscience: Hoboken, New Jersey, 2007.

2. Lindstrom, U. M., Ed. Organic Reactions in Water; Blackwell Publishing: Oxford, 2007.

3. Haggin, J. Chem. Eng. News 1993, 71, 23-27.

4. Togni, A., Grützmacher, H., Eds. Catalytic Heterofunctionalisation; Wiley-VCH Verlag GmbH: Weinheim, 2001.

5. Beller, M.; Seayad, J.; Tillack, A.; Jiao, H. Angew. Chem. Int. Ed. 2004, 43, 3368-3398.

6. Constable, D. J. C.; Dunn, P. J.; Hayler, J. D.; Humphrey, G. R.; Leazer, Jr., J. L. et al. Green Chem. 2007, 9, 411-420.

7. Hintermann, L. Top. Organomet. Chem. 2010, 31, 123-155.

8. Trost, B. M. Angew. Chem. Int. Ed. 1995, 34, 259-281.

9. Burns, N.; Baran, P.; Hoffmann, R. Angew. Chem. Int. Ed. 2009, 48, 2854-2867.

10. Markownikoff, W. Justus Liebigs Ann. Chem. 1870, 153, 228-259.

11. Weissermel, K.; Arpe, H.-J. Industrial Organic Chemistry; VCH Publishers, Inc.: New York, NY, 1997.

12. Dong, G.; Teo, P.; Wickens, Z.; Grubbs, R. Science 2011, 333, 1609-1612.

13. Kano, S.; Tanaka, Y.; Hibino, S. J. Chem. Soc., Chem. Commun., 1980, 414-415.

14. Ogo, S.; Uehara, K.; Abura, T.; Watanabe, Y.; Fukuzumi, S. J. Am. Chem. Soc. 2004, 126, 16520-16527.

15. Tachinami, T.; Nishimura, T.; Ushimaru, R.; Noyori, R.; Naka, H. J. Am. Chem. Soc. 2013, 135, 50-53.

16. Tokunaga, M.; Wakatsuki, Y. Angew. Chem. Int. Ed. 1998, 37, 2867-2869.

17. Labonne, A.; Kribber, T.; Hintermann, L. Org. Lett. 2006, 8, 5853-5856.

18. Grotjahn, D.; Lev, D. J. Am. Chem. Soc. 2004, 126, 12232-12233.

19. Li, L.; Herzon, S. J. Am. Chem. Soc. 2012, 134, 17376-17379.

20. Zhang, Z.; Du Lee, S.; Fisher, A.; Widenhoefer, R. Tetrahedron 2009, 65, 1794-1798. 
21. Kukushkin, V.; Pombeiro, A. Inorg. Chim. Acta. 2005, 358, 1-21.

22. García-Álvarez, R.; Crochet, P.; Cadierno, V. Green Chem. 2013, 15, 46-66.

23. Ahmed, T. J.; Knapp, S. M. M.; Tyler, D. R. Coord. Chem. Rev. 2011, 255, 949-974.

24. Tomás-Mendivil, E.; García-Álvarez, R.; Vidal, C.; Crochet, P.; Cadierno, V. ACS Catal. 2014, 4, 1901-1910.

25. Goto, A.; Endo, K.; Saito, S. Angew. Chem. Int. Ed. 2008, 47, 3607-3609.

26. Daw, P.; Sinha, A.; Rahaman, S. M. W.; Dinda, S.; Bera, J. K. Organometallics 2012, 31, 3790-3797.

27. Ghaffar, T.; Parkins, A. W. Tetrahedron Lett. 1995, 36, 8657-8660.

28. Jiang, X.; Williams, N.; De Brabander, J. K. Org. Lett. 2007, 9, 227-230.

29. Fulton, J. R.; Holland, A.; Fox, D.; Bergman, R. Acc. Chem. Res. 2002, 35, $44-56$.

30. Kundu, S.; Choi, J.; Wang, D.; Choliy, Y.; Emge, T. et al. J. Am. Chem. Soc. 2013, 135, 5127-5143.

31. Ozerov, O. Chem. Soc. Rev. 2009, 38, 83-88.

32. Blum, O.; Milstein, D. J. Am. Chem. Soc. 2002, 124, 11456-11467.

33. Gillard, R. D.; Heaton, B. T.; Vaughan, D. H. J. Chem. Soc. A 1970, 3126-3130.

34. Yoshida, T.; Matsuda, T.; Okano, T.; Kitani, T.; Otsuka, S. J. Am. Chem. Soc. 1979, 101, 2027-2038.

35. Sevov, C.; Hartwig, J. J. Am. Chem. Soc. 2013, 135, 2116-2119.

36. Zhao, J.; Goldman, A.; Hartwig, J. Science 2005, 307, 1080-1082.

37. Kläring, P.; Pahl, S.; Braun, T.; Penner, A. Dalton Trans. 2011, 40, 6785-6791.

38. Dorta, R.; Togni, A. Organometallics 1998, 17, 3423-3428.

39. Morales-Morales, D.; Lee, D. W.; Wang, Z.; Jensen, C. M. Organometallics 2001, 20, 1144-1147.

40. Crabtree, R. H. The Organometallic Chemistry of the Transition Metals, 4th ed.; John Wiley \& Sons, Inc.: Hoboken, New Jersey, 2005.

41. Hanley, P.; Hartwig, J. Angew. Chem. Int. Ed. 2013, 52, 8510-8525.

42. Tye, J.; Hartwig, J. J. Am. Chem. Soc. 2009, 131, 14703-14712.

43. Hanley, P.; Marković, D.; Hartwig, J. J. Am. Chem. Soc. 2010, 132, 6302-6303.

44. Bryndza, H.; Domaille, P.; Tam, W.; Fong, L. Polyhedron 1988, 1441-1452.

45. Zhao, P.; Incarvito, C.; Hartwig, J. J. Am. Chem. Soc. 2006, 128, 9642-9643.

46. Hartwig, J. Inorg. Chem. 2007, 46, 1936-1947.

47. Bryndza, H. E.; Tam, W. Chem. Rev. 1988, 88, 1163-1188.

48. Glueck, D. S.; Winslow, L. J. N.; Bergman, R. G. Organometallics 1991, 10, 1462-1479. 
49. Müller, T.; Beller, M. Chem. Rev. 1998, 98, 675-704.

50. Albrecht, M.; Lindner, M. M. Dalton Trans. 2011, 40, 8733-8744.

51. Kohl, S.; Weiner, L.; Schwartsburd, L.; Konstantinovski, L.; Shimon, L. et al. Science 2009, 324, 74-77.

52. Sevov, C.; Zhou, J.; Hartwig, J. J. Am. Chem. Soc. 2012, 134, 11960-11963.

53. Choi, J.; Amy, M.; Brookhart, M.; Goldman, A. Chem. Rev. 2011, 111, 17611779 .

54. Musaev, D.; Morokuma, K. J. Am. Chem. Soc. 1995, 117, 799-805.

55. Khaskin, E.; Iron, M.; Shimon, L.; Zhang, J.; Milstein, D. J. Am. Chem. Soc. 2010, 132, 8542-8543.

56. Kang, P.; Meyer, T. J.; Brookhart, M. Chem. Sci. 2013, 4, 3497-3502.

57. Hetterscheid, D.; van der Vlugt, J.; de Bruin, B.; Reek, J. Angew. Chem. Int. Ed. 2009, 48, 8178-8181.

58. van der Vlugt, J. Chem. Soc. Rev. 2010, 39, 2302-2322.

59. Ostapowicz, T.; Merkens, C.; Hölscher, M.; Klankermayer, J.; Leitner, W. J. Am. Chem. Soc. 2013, 135, 2104-2107.

60. Grützmacher, H. Angew. Chem. Int. Ed. 2008, 47, 1814-1818.

61. Knapp, S. M. M.; Sherbow, T. J.; Yelle, R. B.; Zakharov, L. N.; Juliette, J. J. et al. Organometallics 2013, 32, 824-834.

62. Berners-Price, S. J.; Bowen, R. J.; Galettis, P.; Healy, P. C.; McKeage, M. J. Coord. Chem. Rev. 1999, 185, 823-836.

63. Newkome, G. Chem. Rev. 1993, 93, 2067-2089.

64. Jain, V.; Jakkal, V.; Bohra, R. J. Organomet. Chem. 1990, 389, 417-426.

65. Bowen, R. J.; Garner, A.; Berners-Price, S. J.; Jenkins, I. D.; Sue, R. E. J. Organomet. Chem. 1998, 554, 181-184.

66. García-Álvarez, R.; García-Garrido, S. E.; Díez, J.; Crochet, P.; Cadierno, V. Eur. J. Inorg. Chem. 2012, 2012, 4218-4230.

67. Grotjahn, D.; Lev, D. J. Am. Chem. Soc. 2004, 126, 12232-12233.

68. Grotjahn, D.; Incarvito, C.; Rheingold, A. Angew. Chem. Int. Ed. 2001, 40, 3884-3887.

69. Grotjahn, D.; Valentín, M.; Kragulj, E.; Lev, D.; Erdogan, G. et al. J. Am. Chem. Soc. 2008, 130, 20-21.

70. Oshiki, T.; Yamashita, H.; Sawada, K.; Utsunomiya, M.; Takahashi, K. et al. Organometallics 2005, 24, 6287-6290.

71. Gunanathan, C.; Milstein, D. Acc. Chem. Res. 2011, 44, 588-602.

72. Milstein, D. Top. Catal. 2010, 53, 915-923.

73. Vergnaud, J.; Grellier, M.; Bouhadir, G.; Vendier, L.; Sabo-Etienne, S. et al. Organometallics 2008, 27, 1140-1146. 
74. Bontemps, S.; Bouhadir, G.; Miqueu, K.; Bourissou, D. J. Am. Chem. Soc. 2006, 128, 12056-12057.

75. Anderson, B.; Spencer, J. Chem. Eur. J. 2014, 20, 6421-6432.

76. Yano, T.; Moroe, Y.; Yamashita, M.; Nozaki, K. Chem. Lett. 2008, 37, 13001301.

77. Kawatsura, M.; Hartwig, J. F. Organometallics 2001, 20, 1960-1964.

78. Dahlhoff, W.; Nelson, S. J. Chem. Soc. A 1971, 2184-2190.

79. Hermann, D.; Gandelman, M.; Rozenberg, H.; Shimon, L. J. W.; Milstein, D. Organometallics 2002, 21, 812-818.

80. Doyle, L. R.; Heath, A.; Low, C. H.; Ashley, A. E. Adv. Synth. Catal. 2014, 356, 603-608.

81. Abada, S.; Lecointre, A.; Elhabiri, M.; Charbonnière, L. Dalton Trans. 2010, 39, 9055-9062.

82. Su, H.; Wu, C.; Zhu, J.; Miao, T.; Wang, D. et al. Dalton Trans. 2012, 41, 14480-14483.

83. Pickett, T. E.; Roca, F. X.; Richards, C. J. J. Org. Chem. 2003, 68, 2592-2599.

84. Peterson, M. A.; Mitchell, J. R. J. Org. Chem. 1997, 62, 8237-8239.

85. Imamoto, T.; Saitoh, Y.; Koide, A.; Ogura, T.; Yoshida, K. Angew. Chem. 2007, 119, 8790-8793.

86. Staubitz, A.; Robertson, A. P.; Sloan, M. E.; Manners, I. Chem. Rev. 2010, 110, 4023-4078.

87. Brunel, J.; Faure, B.; Maffei, M. Coord. Chem. Rev. 1998, 665-698.

88. Allan, K. M. Hybrid P,E Ligands. Synthesis, Coordination Chemistry and Catalysis. Ph.D. thesis, Victoria University of Wellington, 2014.

89. Gilbert-Wilson, R.; Field, L. D.; Bhadbhade, M. M. Inorg. Chem. 2012, 51, 3239-3246.

90. Jana, R.; Chakraborty, S.; Blacque, O.; Berke, H. Eur. J. Inorg. Chem 2013, $4574-4584$.

91. Allen, D. W. In Organophosphorus Chemistry; Tebby, J., Loakes, D., Allen, D., Eds.; The Royal Society of Chemistry, 2014; Vol. 43; pp 1-51.

92. Bartocha, B.; Gray, Y. Z. Naturforsch. 1959, 14, 809-811.

93. Lassalle, L.; Legoupy, S.; Guillemin, J. Organometallics 1996, 15, 3466-3469.

94. Ramsden, H. E. Vinylphosphorus compounds and method of preparation. 1964; US Patent 3,160,666.

95. Monkowius, U.; Nogai, S.; Schmidbaur, H. Organometallics 2003, 22, 145-152.

96. Bhattacharya, A.; Thyagarajan, G. Chem. Rev. 1981, 81, 415-430.

97. Adams, J. J.; Lau, A.; Arulsamy, N.; Roddick, D. M. Inorg. Chem. 2007, 46, $11328-11334$. 
98. Gan, X.; Binyamin, I.; Rapko, B. M.; Fox, J.; Duesler, E. N. et al. Polyhedron 2006, 25, 3387-3392.

99. Byeon, S. R.; Lee, J. H.; Sohn, J. H.; Kim, D. C.; Shin, K. J. et al. Bioorg. Med. Chem. Lett. 2007, 17, 1466-1470.

100. Tavtorkin, A. N.; Toloraya, S. A.; Nifant'ev, E. E.; Nifant'ev, I. E. Tetrahedron Lett. 2011, 52, 824-825.

101. Harvey, S.; Junk, P. C.; Raston, C. L.; Salem, G. J. Org. Chem. 1988, 53, $3134-3140$.

102. Miller, A.; Labinger, J.; Bercaw, J. J. Am. Chem. Soc. 2008, 130, 11874-11875.

103. Vaughan, T. F. Late Transition Metal Complexes of Pyridyldiphosphines. Ph.D. thesis, Victoria University of Wellington, 2014.

104. Schlosser, M. In Organometallics in Synthesis; Schlosser, M., Ed.; Wiley: Chichester, UK, 1994.

105. Uchida, Y.; Kawai, M.; Masauji, H.; Oae, S. Heteroatom Chemistry 1993, 4, 421-426.

106. Uchida, Y.; Kozawa, H. Tetrahedron Lett. 1989, 30, 6365-6368.

107. Birkholz, M.; Dubrovina, N. V.; Jiao, H.; Michalik, D.; Holz, J. et al. Chem. Eur. J. 2007, 13, 5896-5907.

108. Brück, A.; Ruhland, K. Organometallics 2009, 28, 6383-6401.

109. Giannoccaro, P.; Vasapollo, G.; Sacco, A. J. Chem. Soc., Chem. Commun. 1980, 1136-1137.

110. Hahn, C.; Spiegler, M.; Herdtweck, E.; Taube, R. Eur. J. Inorg. Chem. 1999, 1999, 435-440.

111. Hahn, C.; Sieler, J.; Taube, R. Polyhedron 1998, 17, 1183-1193.

112. Sablong, R.; Osborn, J. A. Tetrahedron Letters 1996, 37, 4937-4940.

113. Tolman, C. A. Chemical Reviews 1977, 77, 313-348.

114. Beckmann, U.; Süslüyan, D.; Kunz, P. C. Phosphorus, Sulfur, Silicon Relat. Elem. 2011, 186, 2061-2070.

115. Murray, C.; Norris, D.; Bawendi, M. J. Am. Chem. Soc. 1993, 115, 8706-8715.

116. Ludwig, M. Secondary phosphine selenides and process for preparing. 1970; WO Patent App. PCT/GB2010/052,214.

117. http://www.rsc.org/chemistryworld/2014/11/ selenium-dioxide-selenonyl-podcast, 2014.

118. Allen, D.; Taylor, B. J. Chem. Soc., Dalton Trans. 1982, 51-54.

119. Buhling, A.; Kamer, P.; van Leeuwen, P. Organometallics 1997, 16, 3027-3037.

120. Schrock, R.; Osborn, J. J. Am. Chem. Soc. 1976, 98, 2134-2143.

121. Wiebus, E.; Cornils, B. Chem. Ing. Tech. 1994, 66, 916-923.

122. Hahn, C.; Sieler, J.; Taube, R. Chem. Ber./Recueil 1997, 130, 939-945. 
123. Vasapollo, G.; Giannoccaro, P.; Nobile, C. F.; Sacco, A. Inorg. Chim. Acta. 1981, 48, 125-128.

124. Pregosin, P. S. NMR in Organometallic Chemistry; Wiley-VCH Verlag GmbH: Weinheim, 2012.

125. Andersson, P. G., Ed. Iridium Catalysis; Topics in Organometallic Chemistry; Springer, 2011; Vol. 34.

126. Feller, M.; E, B.; Gupta, T.; Shimon, L. Inorg. Chem. 2007, 46, 10479-10490.

127. Borisenko, A.; Sergeyev, N.; Ustynyuk, Y. Mol. Phys. 1971, 22, 715-719.

128. Hanson, S. K.; Heinekey, D. M.; Goldberg, K. I. Organometallics 2008, 27, 1454-1463.

129. Windmüller, B.; Nürnberg, O.; Wolf, J. Eur. J. Inorg. Chem. 1999, 613-619.

130. Feller, M.; Iron, M. A.; Shimon, L. J. W.; Diskin-Posner, Y.; Leitus, G. et al. J. Am. Chem. Soc. 2008, 130, 14374-14375.

131. Smith, D. A.; Herbert, D. E.; Walensky, J. R.; Ozerov, O. V. Organometallics 2013, 32, 2050-2058.

132. Semproni, S. P.; Milsmann, C.; Chirik, P. J. J. Am. Chem. Soc. 2014, 136, 9211-9224.

133. Haibach, M. C.; Guan, C.; Wang, D. Y.; Li, B.; Lease, N. et al. J. Am. Chem. Soc. 2013, 135, 15062-15070.

134. Coetzee, J.; Eastham, G.; Slawin, A. Dalton Trans. 2015, 44, 1585-1591.

135. Eyal, B.; Leitus, G.; Shimon, L.; Milstein, D. J. Am. Chem. Soc. 2006, 128, 15390-15391.

136. Rybtchinski, B.; Ben-David, Y.; Milstein, D. Organometallics 1997, 16, 37863793.

137. Tanaka, R.; Yamashita, M.; Nozaki, K. J. Am. Chem. Soc. 2009, 131, 14168 14169.

138. Olmos, M. E.; Schier, A.; Schmidbaur, H. Z. Naturforsch. 1997, 52, 203-208.

139. Eyal, B.; Gandelman, M.; Rozenberg, H.; Shimon, L. J.; Milstein, D. J. Am. Chem. Soc. 2003, 125, 4714-4715.

140. Sevov, C. S.; Hartwig, J. F. J. Am. Chem. Soc. 2013, 135, 9303-9306.

141. Gloaguen, Y.; Jacobs, W.; de Bruin, B.; Lutz, M.; van der Vlugt, J. I. Inorg. Chem. 2013, 52, 1682-1684.

142. Yamashita, M.; Moroe, Y.; Yano, T.; Nozaki, K. Inorg. Chim. Acta. 2011, 369, $15-18$.

143. Kline, M.; Cheatham, S. Magn. Reson. Chem. 2003, 41, 307-314.

144. Adams, J.; Arulsamy, N.; Roddick, D. Organometallics 2011, 30, 697-711.

145. Wormald, C. J. J. Chem. Thermodyn. 2002, 34, 1639-1646.

146. Klabunde, K. J.; Burton, D. J. J. Am. Chem. Soc. 1972, 94, 5985. 
147. Scharlin, P. Acta Chem. Scand. A 1987, 41, 480-483.

148. Wesener, J.; Moskau, D.; Guenther, H. J. Am. Chem. Soc. 1985, 107, 73077311.

149. Albright, T. A.; Hoffmann, R.; Thibeault, J. C.; Thorn, D. L. J. Am. Chem. Soc. 1979, 101, 3801-3812.

150. Adams, J.; Arulsamy, N.; Roddick, D. Polyhedron 2014, 84, 209-215.

151. Hlatky, G.; Crabtree, R. Coord. Chem. Rev. 1985, 65, 1-48.

152. Tanaka, R.; Yamashita, M.; Chung, L. W.; Morokuma, K.; Nozaki, K. Organometallics 2011, 30, 6742-6750.

153. Caballero, A.; Jalón, F. Chem. Commun. 1998, 1879-1880.

154. Hull, J. F.; Himeda, Y.; Wang, W.-H.; Hashiguchi, B.; Periana, R. et al. Nat. Chem. 2012, 4, 383-388.

155. Li, W.; Xie, J.; Lin, H.; Zhou, Q. Green Chem. 2012, 14, 2388-2390.

156. Balaraman, E.; Khaskin, E.; Leitus, G.; Milstein, D. Nat. Chem. 2013, 5, $122-125$.

157. Sidera, M.; Costa, A. M.; Vilarrasa, J. Org. Lett. 2011, 13, 4934-4937.

158. Arrowsmith, M.; Crimmin, M. R.; Hill, M. S.; Lomas, S. L.; MacDougall, D. J. et al. Organometallics 2013, 32, 4961-4972.

159. Villarino, L.; Garcia-Fandino, R.; Lopez, F.; Mascarenas, J. L. Org. Lett. 2012, 14, 2996-2999.

160. Ohmura, T.; Yorozuya, S.; Yamamoto, Y.; Miyaura, N. Organometallics 2000, 19, 365-367.

161. Shirakura, M.; Suginome, M. J. Am. Chem. Soc. 2008, 130, 5410-5411.

162. Nishimura, T.; Guo, X.-X.; Uchiyama, N.; Katoh, T.; Hayashi, T. J. Am. Chem. Soc. 2008, 130, 1576-1577.

163. Powala, B.; Pietraszuk, C. Catal. Lett. 2014, 144, 413-418.

164. Fu, X.; Yu, S.; Fan, G.; Liu, Y.; Li, Y. Organometallics 2012, 31, 531-534.

165. Ghosh, R.; Zhang, X.; Achord, P.; Emge, T. J.; Krogh-Jespersen, K. et al. J. Am. Chem. Soc. 2007, 129, 853-866.

166. Yoshida, T.; Matsuda, T.; Okano, T.; Kitani, T.; Otsuka, S. J. Am. Chem. Soc. 1979, 101, 2027-2038.

167. Chin, C. S.; Kim, S. Y.; Joo, K.-S.; Won, G.; Chong, D. Bull. Korean Chem. Soc. 1999, 20, 535-538.

168. Crestani, M. G.; Steffen, A.; Kenwright, A. M.; Batsanov, A. S.; Howard, J. A. K. et al. Organometallics 2009, 28, 2904-2914.

169. King, R.; Masler, W. J. Am. Chem. Soc. 1977, 99, 4001-4008.

170. Eastham, G.; Waugh, M.; Pringle, P.; Turner, T. 2011; WO Patent App. $\mathrm{PCT} / \mathrm{GB} 2010 / 052,214$. 
171. Anderson, B. G. Late Transition Metal Complexes of Pentafluorophenylphosphino-Pincer Ligands. Ph.D. thesis, Victoria University of Wellington, 2012.

172. Dervisi, A.; Carcedo, C.; Ooi, L. Adv. Synth. Catal. 2006, 348, 175-183.

173. Tajuddin, H.; Harrisson, P.; Bitterlich, B.; Collings, J. C.; Sim, N. et al. Chem. Sci. 2012, 3, 3505-3515.

174. Uson, R.; Oro, L. A.; Cabeza, J. A.; Bryndza, H. E.; Stepro, M. P. In Inorganic Syntheses; Kirschner, S., Ed.; John Wiley \& Sons, Inc.: Hoboken, New Jersey, 1985; Vol. 23; pp 126-130.

175. Herde, J. L.; Lambert, J. C.; Senoff, C. V.; Cushing, M. A. In Cyclooctene and 1,5-Cyclooctadiene Complexes of Iridium(I), in Inorganic Syntheses; Parshall, G. W., Ed.; John Wiley \& Sons, Inc.: Hoboken, NJ, USA., 1974; Vol. 15; pp 45-64.

176. Fulmer, G.; Miller, A.; Sherden, N.; Gottlieb, H. Organometallics 2010, 29, 2176-2179.

177. Dolomanov, O.; Bourhis, L.; Gildea, R.; Howard, J.; Puschmann, H. J. Appl. Cryst. 2009, 42, 339-341.

178. Palatinus, L.; Chapuis, G. J. Appl. Cryst. 2007, 786-790.

179. Palatinus, L.; van der Lee, A. J. Appl. Cryst. 2008, 41, 975-984.

180. Palatinus, L.; Prathapa, S. J.; van Smaalen, S. J. Appl. Cryst. 2012, 45, 575-580. 